\title{
Métodos auto-organizáveis para segmentação de imagens
}

\author{
Patricia Rufino Oliveira
}

Orientadora: Profa. Dra. Roseli Aparecida Francelin Romero

Tese apresentada ao Instituto de Ciências Matemáticas e de Computação - ICMC-USP, como parte dos requisitos para obtenção do título de Doutor em Ciências -- Ciências de Computação e Matemática Computacional.

USP - São Carlos

Novembro de 2004 


\section{Agradecimentos}

A Deus por mais essa realização e por todas as oportunidades que tive até hoje.

A meus queridos pais. Antônio e Socorro Oliveira que com tanto amor me educaram e me ensinam fundamentos que levarei comigo para sempre. A eles agradeço pelos apoios morais, emocionais e financeiros incondicionais.

À minha orientadora. Roseli Aparecida Francelin Romero, por ter confiado na minha capacidade de conduzir esse e outros projetos; pela constante motivação e perseverança com que encarou os desafios deste trabalho e pelo prazer das longas conversas que tivemos ao longo desses anos.

Aos meus irmãos Gisela e Matheus Oliveira pelo carinho, apoio e compreensão, e principalmente por entendercm os momentos de minha ausência.

Ao pesquisador Dr. Aapo Hyvärinen, supervisor do meu estágio na universidade Helsinki University of Technology. Finlândia. que contribuiu com várias idéias implementadas neste trabalho, pelo conhecimento compartilhado e pela oportunidade que tive em conhecer seu centro de pesquisa.

Aos meus amigos de fé, Andréia e Isabel Rodrigues e Júlio Menochelli pela companhia fiel ao longo desta e de outras jornadas.

Aos meus avós e tios. pelo incentivo constante, e pelos momentos marcantes e felizes.

Aos amigos que conheci em São Carlos e que fizeram e fazem toda a diferença, Thais Aiza, Sandra de Góes. Sidney Oliveira, Daylton Romão, Karina Salemi, Renato Pagotto e Jaqueline Pugliesi.

A Eduardo J. Spinosa, pela valiosa ajuda na revisão de partes deste trabalho, e pela amizade.

A José Flávio Vicentini pela oportunidade de termos desenvolvido alguns trabalhos em comum, pela amizade e momentos de descontração.

A todos os colegas do Labic, em especial, a Katti Faceli, Gedson Faria, Edson Melanda, Ana Lorena, Huei Lee e Mariza Ferro pelos momentos compartilhados, dentro e fora da USP.

Aos professores Maria Carolina Monard e André de Carvalho pelo apoio recebido desde a época do meu mestrado.

A todos funcionários do ICMC-USP, em especial, à Beth (secretaria de pós-graduação), Paulinho (áudio-visual). Maria, Sandra e Rose (biblioteca) pela simpatia e compreensão com que sempre me atenderam.

A CAPES pelo apoio financeiro no Brasil e no exterior. 


\section{Resumo}

Segmentação de imagens é um dos problemas mais investigados na área de computação visual. A complexidade desse problema varia de acordo com o tipo de aplicação. Em geral, o objetivo é dividir a imagem $\mathrm{cm}$ regiōes que apresentem propriedades similares. No presente trabalho, métodos auto-organizáveis para classificação não supervisionada e agrupamento de padrōes são utilizados $\mathrm{cm}$ tarefas de segmentação. O primeiro modelo refere-se à rede neural Fuzzy ART e o segundo é o Modelo de Misturas ICA (ICAMM) que faz uso da técnica ICA (Análise de Componentes Independentes) para descrever os dados em cada classe.

Além da avaliação de desempenho dos modelos auto-organizáveis utilizados, foram propostas melhorias nos resultados de segmentação por meio da incorporação de técnicas de préprocessamento de imagens, que sejam capazes de tratar questōes referentes à presença de ruídos, suavização de imagens e realce de bordas, de modo que as imagens se tornem mais adequadas ao processo de segmentação, tornando-o mais eficiente. Com esse objetivo, foi proposta uma metodologia para pré-processamento de imagens, que combina o método Sparse Code Shrinkage, para redução de ruídos e suavização da imagem, e o detector de bordas de Sobel, que tem a função de restaurar as bordas que foram borradas pelo processo de suavização.

Outra contribuição original deste trabalho refere-se ao desenvolvimento do método EICAMM, que surgiu por meio da proposta de melhorias incorporadas ao modelo ICAMM, levando em consideração algumas limitações do método original e análises de como este poderia se tornar mais eficiente.

Finalmente, unificando as duas principais contribuições originais desta tese, o método EICAMM foi utilizado na segmentação de imagens nas suas versões originais e pré-processadas pela metodologia proposta neste trabalho, tendo apresentado resultados de segmentação satisfatórios. 


\section{Abstract}

Image segmentation is one of most. investigated problems in computer vision. Its complexity can vary according to the kind of application. In general, the goal is to divide an image into regions with similar properties. In this work, self-organizing methods for unsupervised classification and clustering are applied in image scgmentation tasks. The first self-organizing model is the Fuzzy ART neural network and the other one is the ICA Mixture Model (ICAMM), which uses ICA method to deseribe data in each class.

Beside the performance evaluation regarding the considered mothods, some improvements on the segmentation results oltained by these techniques were proposed by incorporating some image preprocessing methods. Such methods were able to handle some questions regarding to presence of noise, inage smoothing and edge enhancement, in a way that makes an image more suited to be processed by an image segmentation technique, which can become more efficient. Aiming this, a preprocessing methodology was proposed in this work that combines Sparse Code Shrinkage method for image derroise to the Sobel Edge Detector, which is applied to recover edges that were blured by an excessive smoothing.

Another original contribution of this work refers to the development of EICAMM, which was built by proposing some modifications on ICAMM, considering some limitations on the original method and analysis on how it should be modified to become more efficient.

Finally, unifying the two main contributions of this thesis, the EICAMM nethod was applied for segmenting some images in these original and preprocessed versions, obtained by the proposed preprocessing methodology: Such system has showed satisfactory image segmentation results. 


\section{Conteúdo}

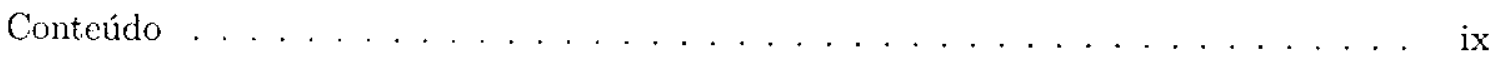

Lista de Figuras . . . . . . . . . . . . . . . . . . . . xi

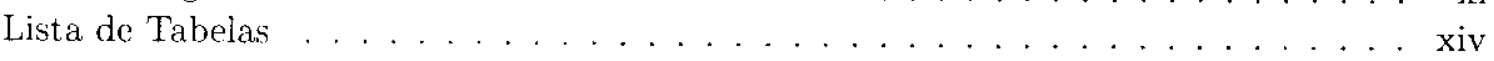

Lista de Abreviaturas xvii

1 Introdução 1

1.1 Contexto e Motivagão . . . . . . . . . . . . . . . . 1

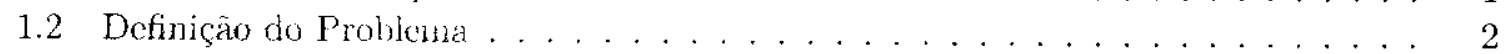

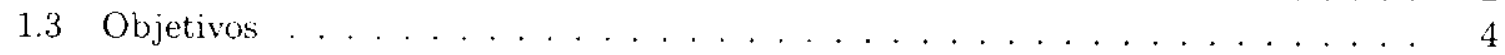

1.4 Organizaçào do Trabalho . . . . . . . . . . . . . . . . . 4

2 Definiçōes 7

2.1 Consideraçoes Iniciais $\ldots \ldots \ldots \ldots \ldots$

2.2 Definiçoes e Propriedades de Vetores e Matrizes . . . . . . . . . . . . . . . 7

2.2.1 Norma: Distância Euclidiana e Ortogonalidade de Vetores . . . . . . . . 7

2.2 .2 Matrizes Identidado e Diagonal . . . . . . . . . . . . . . . 8

2.2 .3 Transposição e Inversão de Matrizes . . . . . . . . . . . . . . . 8

2.2.4 Natriz Simétrica e Ortogonal . . . . . . . . . . . . . . . . 8

2.2 .5 Determinaute e Cofator . . . . . . . . . . . . . . 8

2.2 .6 Matriz Adjunta . . . . . . . . . . . . . . . . . . . . 9

2.2 .7 Matrizes de Derivadas Parciais . . . . . . . . . . . . . 9

2.3 Distribuçós c Densidades de Probabilidade . . . . . . . . . . . . . . . 10

2.3 .1 Distribuição de uma Variável Aleatória . . . . . . . . . . . . . 10

2.3.2 Distribuição de um Vetor Aleatório . . . . . . . . . . . . . . . . . 11

2.3.3 Distribuicoes Conjunta e Marginal . . . . . . . . . . . . . . . . 11

2.4 Esperança Matcmática e Momentos . . . . . . . . . . . . . . . . . 12

2.4 .1 Definigoes e Propriedades Gerais . . . . . . . . . . . . . . . . 12

2.4 .2 Vetor de Média c Matriz de Correlação . . . . . . . . . . . . . . . 13

2.4 .3 Matriz de Covariancia . . . . . . . . . . . . . . . . . . 14

2.4.4 Estimativas de Esperanças . . . . . . . . . . . . . . . . 15

2.5 Não Correlação e Branqueamento . . . . . . . . . . . . . . . . 16

2.6 Independência Estatística . . . . . . . . . . . . . . . . . . 17

2.7 Densidades Condicionais e Regra de Bayes . . . . . . . . . . . . . . . . . . . 19

2.8 A Densidade Gaussiana Multivariada . . . . . . . . . . . . . . . . . 20

2.8 .1 Propriedades da Densidade Gaussiana . . . . . . . . . . . . . 20

2.9 Outros Conceitos Iniportantes . . . . . . . . . . . . . . . . . . . . 21 
2.10 Consideraçoes Finais . . . . . . . . . . . . . . . . . . . . . . . 23

3 Métodos de Análise Multivariada e Redes Neurais Artificiais $\quad 25$

3.1 Consideraçôes Iniciais . . . . . . . . . . . . . . . . . . . . . . . 25

3.2 Métodos de Análise Multivariada . . . . . . . . . . . . . . . . . . . . . 26

3.2.1 Análisc de Componentes Principais (PCA) . . . . . . . . . . . . . 27

3.2 .2 Técnicas de Agrupamento (Clustering) . . . . . . . . . . . . . . . 31

3.3 Redes Neurais Artificiais . . . . . . . . . . . . . . . . . . . . . . 33

3.3.1 Processo de Aprendizado para Redes Neurais . . . . . . . . . . . . . . . 33

3.3 .2 Modolos da Família ART . . . . . . . . . . . . . . . . . . . . . . . . . . . . . . . . . . . . . .

3.3 .3 Rede Neural PCA Adaptativa . . . . . . . . . . . . . 51

3.4 Consideraçöes Finais . . . . . . . . . . . . . . . . . . . . . . 55

4 Análise de Componentes Independentes $\quad \mathbf{5 9}$

4.1 Consideraçoes Iniciais . . . . . . . . . . . . . . . . . . . . . . . . 59

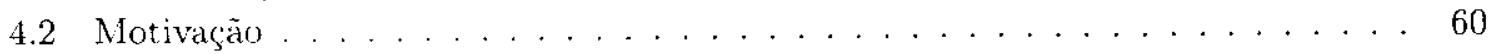

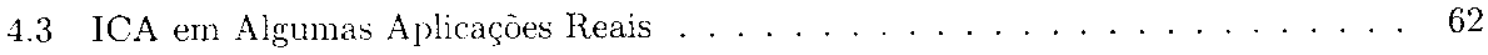

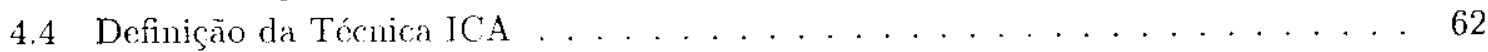

4.5 Suposicoes e Restricoes da ICA . . . . . . . . . . . . . . . . . . 63

4.6 Ambiguidades do Nodelo ICA . . . . . . . . . . . . . . . . . . . . . 65

4.7 Não Correlaçào. Branqueamento e Independência . . . . . . . . . . . . . . . . . 66

4.8 Abordagem IC A baseada na Teoria da Informação . . . . . . . . . . . . . . 67

4.8.1 Formulação do Problema ICA Utilizando Conceitos de Teoria de Informação 68

4.8 .2 Abordagem Infomax para ICA . . . . . . . . . . . . . . . . . 69

4.8.3 Regras de Aprendizado ICA para uma Rede Neural com uma Entrada e uma Saída. . . . . . . . . . . . . . . . . 72

4.8.4 Regras de Aprendizado ICA para uma Rede Neural com $N$ Entradas e $N$

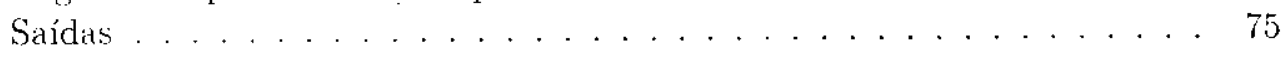

4.8.5 Equivalchncia entre Maximização de Informação e Estimação por Máxima

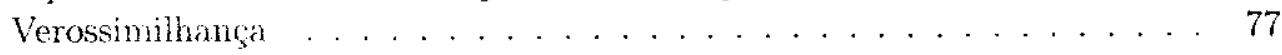

4.9 Maximizaça da Negentropia . . . . . . . . . . . . . . . . . . . . . . . . . . . . . . . . . . . . . . . . . . .

4.9 .1 O Algoritmo FastICA . . . . . . . . . . . . . . . . . 79

4.10 Consideragöes Finais . . . . . . . . . . . . . . . . . . . . . . . 80

5 Pré-Processamento de Imagens 83

5.1 Consideraçoes Iniciajs . . . . . . . . . . . . . . . . . . . . . . . . . 83

5.2 Método Sparse Code Shrinkage para Redução de Ruídos em Imagens . . . . . . . 84

5.2 .1 Reduçào do Ruídos cm Variáveis Alcatórias Não-Gaussianas . . . . . . . . 86

5.2 .2 Modelagem para Densidades Esparsas . . . . . . . . . . . . . . . 86

5.2.3 Transformação dos Dados em Variáveis Esparsas . . . . . . . . . . . . . 87

5.3 Detector de Bordas de Sobel . . . . . . . . . . . . . . . . . . . . . . . . 89

5.4 Metodologia Proposta . . . . . . . . . . . . . . . . . . 90

5.4.1 Exemplo de uma Aplicação da Metodologia Proposta . . . . . . . . . 91

5.5 Consideraçós Finais . . . . . . . . . . . . . . . . . . . . . 91 
6 Modelo de Misturas ICA 95

6.1 Consideraçôes Iniciais . . . . . . . . . . . . . . . . . . . . . 95

6.2 Formulação do Nodelo ICAMM . . . . . . . . . . . . . . . . . . . . . 96

6.3 Derivação do Algoritmo ICAMM . . . . . . . . . . . . . . . . . . 9

6.3 .1 Estimação das Matrizes de Bases . . . . . . . . . . . . . . . . . . . . . . . 99

6.3 .2 Regras de Aprendizado do Modelo ICAMM . . . . . . . . . . . . . . . . . . . 103

6.3 .3 Estimação dos Vetores de Bias . . . . . . . . . . . . . . . . . . . . . 103

6.4 Problemas do ICAMM . . . . . . . . . . . . . . . . . . . . . . . . . 104

6.5 Consideraçoes Finais . . . . . . . . . . . . . . . . . . . . . 105

7 Modelo de Misturas ICA Aperfeiçoado (EICAMM) 107

7.1 Consideraçöes Iniciais . . . . . . . . . . . . . . . . . . . 107

7.2 Reformulação do Modelo de Classes . . . . . . . . . . . . . . . . . . 107

7.3 Regra de Aprendizado para os Termos de Bias . . . . . . . . . . . . . . . . 108

7.4 Ortogonalizaçào das Matrizes de Bases . . . . . . . . . . . . . . . . . . 109

7.5 Incorporação de Informações de Segunda Derivada . . . . . . . . . . . . . . . . 109

7.5 .1 Método de Newton . . . . . . . . . . . . . . . . . . . 110

7.5 .2 Mćtodo de Levenberg-Marquardt . . . . . . . . . . . . . . . . . 110

7.6 Consideracoes Finais . . . . . . . . . . . . . . . . . . . 111

8 Resultados Experimentais $\quad 113$

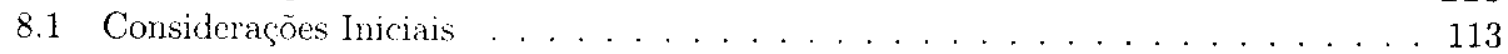

8.2 Experimentos com Dados Simulados . . . . . . . . . . . . . . . . 114

8.2.1 Descriçào dos Dados Simulados . . . . . . . . . . . . . . . . 114

8.2 .2 Resultados e Discussões . . . . . . . . . . . . . . . . . . . 114

8.2.3 Análise Visual de Resultados . . . . . . . . . . . . . . . . . . . . 116

8.3 Experimentos com o Conjunto de Dados Iris . . . . . . . . . . . . . . 117

8.3.1 Descriça dos Dados . . . . . . . . . . . . . . . . . . 117

8.3 .2 Resultados e Discussões . . . . . . . . . . . . . . . . . . 118

8.4 Experimentos con Inagens . . . . . . . . . . . . . . . . . . . . 119

8.4.1 Descriça dos Dados de Imagens . . . . . . . . . . . . . . . . . 119

8.4.2 Aplicaçào da Metodologia de Pré-Processamento Proposta . . . . . . . . 120

8.4 .3 Experimentos de Segmentação de Imagens . . . . . . . . . . . . . . . 123

8.5 Consideraçôes Finais . . . . . . . . . . . . . . . . . . . . 130

9 Conclusões e Trabalhos Futuros $\quad 133$

9.1 Principais Consideraçöes . . . . . . . . . . . . . . . . . 133

9.2 Principais Contribuiçoes . . . . . . . . . . . . . . . . . . . . . 135

9.3 Propostas de Trabalho Futuros . . . . . . . . . . . . . . . . 136

A Derivação das Regras de Aprendizado ICA $\quad 137$

A.1 Para uma Rede Neural com uma Entrada e uma Saída . . . . . . . . . . . . 137

A.1.1 Função de Transfercencia Logística . . . . . . . . . . . . . . . . 137

A.2 Função de Transferência Tangente Hiperbólica . . . . . . . . . . . . . . . . . 139

A.3 Para uma Rede Neural com $N$ Entradas e $N$ Saídas . . . . . . . . . . . . . 141

$\begin{array}{ll}\text { Referências } & 143\end{array}$ 


\section{Lista de Figuras}

2.1 Função de densidade de probabilidade de uma distribuição gaussiana com média

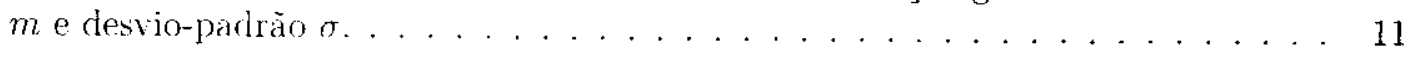

3.1 Estrutura básica de una rede ART . . . . . . . . . . . . . 41

3.2 Arquitetura da rede ART1. . . . . . . . . . . . . . . . . . 44

3.3 Arquitetura da rede Fuzzy ART. . . . . . . . . . . . . . . . 47

3.4 Rede Neural PCA Adaptativa. . . . . . . . . . . . . . . . . . . 52

3.5 Resultados obtidos pelas técnicas de compressão . . . . . . . . . . . . 56

4.1 Exemplo de sinais originais. . . . . . . . . . . . . . . . 61

4.2 Exemplos de misturas de sinais observadas. . . . . . . . . . . . . . . 61

4.3 Estimativas dos sinais do fontes originais . . . . . . . . . . . . . . 61

4.4 Exemplos de funçóes de densidade de probabilidade supergaussianas e subgaussianas 64

4.5 A função logística e sua derivada . . . . . . . . . . . . . . . . 72

4.6 Fluxo ótimo em neurônios sigmoidais . . . . . . . . . . . . . . . . . . . . . 74

5.1 Função de densidade da distribuição de Laplace . . . . . . . . . . . . . . . . . . . 87

5.2 Máscaras de Sobel . . . . . . . . . . . . . . . . . . . . . 90

5.3 Metodologia de pré-processamento proposta . . . . . . . . . . . . . 91

5.4 Exemplo da aplicaçào da metodologia de pré-processamento . . . . . . . . . . 92

8.1 Exemplo de clados simmlados com duas classes laplacianas. . . . . . . . . . . . 115

8.2 Exemplo de dados simmlados com duas classes laplacianas e uma classe uniforme. 115

8.3 Análise visual de resultados dos métodos EICAMM e $k$-médias . . . . . . . . . 118

8.4 Imagens originais utilizadas nos experimentos . . . . . . . . . . . . . . . 121

8.5 Resultados da aplicação da metodologia de pré-processamento proposta . . . . . 122

8.6 Esquema de formatação de padrōes para dados de imagens . . . . . . . . . . . 123

8.7 Resultados de segmentasào da rede neural Fuzzy ART para imagens originais $K=2125$

8.8 Resultados de segmentaçào da rede neural Fuzzy ART para imagens pré-processadas

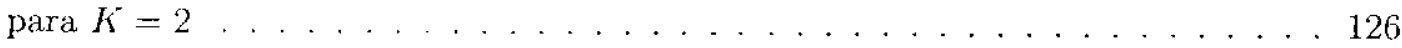

8.9 Resultados de segmentação de imagens utilizando o modelo EICAMM aplicado

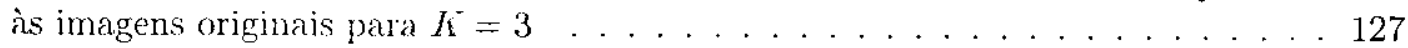

8.10 Resultados de segmentação de imagens utilizando o modelo EICAMM aplicado às imagens pré-processadas para $K=3 \ldots \ldots$. . . . . . . . . . . 128

8.11 Resultados de segmentaçāo de imagens utilizando o modelo EICAMM aplicado às imagens pré-processadas para $K=5 \ldots \ldots \ldots$. . . . . . . . . . . . 129

8.12 Resultados de segmentação de imagens utilizando o algoritmo $k$-médias aplicado às imagens pré-processadas . . . . . . . . . . . . . . . . . . . 130 


\section{Lista de Tabelas}

3.1 Dados para uma PCA. . . . . . . . . . . . . . . . . . 28

3.2 Notação utilizada 1 algoritmo de aprendizado da rede ART1. . . . . . . . . 45

3.3 Inicialização dos parâmetros da rede ART1. . . . . . . . . . . . . . . . 45

3.4 Paràmetros da rede Fuzzy ART. . . . . . . . . . . . . . . . . . 48

3.5 Resultados da PCA clássica para o conjunto de dados Iris. . . . . . . . . . . . 53

3.6 Resultados da rede PCA Adaptativa para o conjunto de dados Iris. . . . . . . . 53

3.7 Resultados de compressão para o JPEG, PCA clássica e rede PCA. . . . . . . 55

8.1 Resultados de classificação para dados simulados. . . . . . . . . . . . . 116

8.2 Resultados de classificasão para o conjunto de dados Iris. . . . . . . . . . . . 119

8.3 Detalhes de descrição das imagens utilizadas nos experimentos. . . . . . . . 120 


\section{Lista de Abreviaturas}

ART

BSS

EICAMM

ICA

ICAMM

JPEG

MLE

MLP

MSE

PCA

RNA

SCS

SOM
Teoria da Ressonância Adaptativa - Adaptive Resonance Theory...........2

Separação Ccga de Fontes- Blind Source Separation ..................6 60

Modelo de Misturas ICA Aperfeiçoado - Enhanced ICA Mixture Model.... 5

Análise de Componentes Independentes - Independent Component Analysis. 2

Modelo de Misturas ICA - ICA Mixture Model ....................... 2

Joint Photographic Expert Group ............................. 54

Estimação por Náxima Verossimilhança - Maximum Likelihood Estimation 68

Perceptron Multi Camadas - Multi Layer Perceptron ................. 35

Erro Quadrático Médio - Mean-Square Error . ..................... 54

Análise de Componentes Principais - Principal Component Analysis ...... 2

Redes Neurais Artificiais ..................................... 26

Sparse Code Shrinkage ......................................2

Mapas Auto-Organizáveis - Self-Organizing Maps................... 37 


\section{Capítulo 1}

\section{Introdução}

\subsection{Contexto e Motivação}

O processo de segmentação ć um passo importante que antecede a manipulação e análise de imagens, sendo o seu principal objetivo separar os objetos de interesse do restante da imagem, para que futuras tarcfas de rcconhecimento e classificação possam ser realizadas com sucesso (Russ 1992), (Pratt 2001).

Vários métodos para segmentação de imagens podem ser encontrados na literatura. Dentre as abordagens clássicas, destacam-se aquelas baseadas em medidas de descontinuidade c/ou similaridade calculadas a partir dos valores de pixels em regiões da imagem (Jain 1989), (Gonzalez \& Woods 1992). (Pratt 2001). Os métodos baseados em descontimuidades tendem a particionar uma imagem por meio da deteç̧ão de pontos isolados, linhas e arestas, de acordo com mudanças abruptas dos valores de pixcls. Já as abordagens baseadas em medidas de similaridade incluem as técnicas de himiarização (Sahoo, Soltani, Wong \& Chen 1988), agrupamento, crescimento de regiōes e divisão e fusâo de regiões (Fu \& Mui 1981).

Outras abordagens. consideradas inteligentes por apresentarem capacidade de aprendizado, generalização, incorporaçăo de conhecimento prévio (a priori) e manipulação de incertezas, tềm sido aplicadas com sucesso em tarefas de segmentação. Dentre as técnicas que se adeçuam a essa catcgoria. merccem destaque a abordagem Bayesiana (Guo \& Ma 2000), (Cheng \& Bouman 2001), modelos de redes neurais artificiais (Bhandarkar, Koh \& Suk 1997), métodos baseados na tcoria de conjuntos nebulosos (fuzzy) (Zhang, Hall \& Goldgof 2002), (Karayiannis \& Pai 1999), (Pham \& Prince 1999), (Pham \& Yan 1999) e técnicas que utilizam propriedades estatísticas das imagens (Tasciyan, Beckmann, Morris \& Smith 2001), (Derrode, Mercier \& Pieczynski 2003). Alguns métodos inteligentes que apresentam capacidade de auto-organização, 
denominados, portanto, auto-organizáveis, são os métodos de enfoque desta tese, podendo-se definir por auto-organização um processo por meio do qual um sistema organiza-se espontaneamente, sem que haja un controle externo exercido pelo ambiente.

Embora vários trabalhos que envolvcm a utilização de métodos auto-organizáveis para segmentar imagens sejam encontrados na literatura (Pham \& Prince 1999), (Lo \& Pei 1999), (Yao, Mignottc, Collct. Galcrne \& Burel 2000), a segmentação automática de imagens ainda é considerada, na maioria das aplicações, uma tarefa difícil. No presente trabalho, modelos autoorganizáveis para classificação não supervisionada e agrupamento de padrões são considerados para utilização e análise em tarefas de segmentação. O primeiro modelo refere-se a uma rede neural baseada na Teoria da Ressonância Adaptativa $(\mathrm{ART})^{1}$ que utiliza fundamentos da teoria de conjuntos nebulosos. sendo, por isso, conhecida como rede neural Fuzzy ART. O segundo modelo é o Modelo de Misturas ICA (ICAMM)², que faz uso da técnica ICA para descrever os dados em cada classe.

Dentre os fatores que podem prejudicar o processo de segmentação, pode-se citar a presença frequente de algum tipo de ruído. adicionado às imagens durante o processo de aquisiçāo $\mathrm{e}$ transmissão das mesmas. c a dificuldade em extrair, a partir de dados de imagens, características que os tornem mais adequados para o processamento computacional. As técnicas de Análise de Componentes Principais (PCA) ${ }^{3}$. Análise de Componentes Independentes (ICA) 4 e o método Sparse Code Shrinkage (SCS) são exemplos de abordagens estatísticas que podem ser utilizadas para solucionar alguns problemas relacionados à redução de dimcnsionalidade, extração de características e supressão de ruídos.

Essas técnicas estatísticas c os modclos auto-organizávcis considerados serão descritos e discutidos ao longo deste trabalho, uma vez que os mesmos foram investigados e utilizados, com o intuito de melhorar o descmpenho de alguns métodos auto-organizáveis para segmentação de imagens.

\subsection{Definição do Problema}

Segmentação de imagens ć um dos problemas mais investigados na área de computação visual. A complexidade desse problema varia de acordo com o tipo de aplicação, sendo que, no caso geral, o objetivo é dividir a imagem em regiōes que apresentem propriedades similares. Essas similaridades podem estar relacionadas, por exemplo, a estruturas estatísticas, propriedades

\footnotetext{
${ }^{1}$ Do original, em inglês. Adaptivc Resonance Theory.

${ }^{2}$ Do original, em ingles, ICA Mixture Model.

${ }^{3}$ Do original, em inglés, Principal Component Analysis.

${ }^{4}$ Do original. em inglês, Independent Component Analysis.
} 
visuais ou geométricas, ou a medidas de distância (similaridade) previamente estabelecidas. Métodos de classificação são adequados para resolver esse tipo de problema, pois as regiões a serem detectadas no processo de segmentação podem ser vistas como classes (ou grupos) às quais os pixels de uma imagem podem ser associados. Dessa forma, pixels pertencentes a uma mesma classe podem representar uma região com propriedades consistentes.

Un problema de classificação de padrões pode ser resolvido computacionalmente por algoritmos supervisionados on nào supervisionados. Em uma classificação supervisionada, cada padrão do conjunto de dados é identificado como sendo um membro de uma classe predefinida. Já um algoritmo de classificação não supervisionada associa cada padrão a uma classe baseando-se somente em estatísticas on em medidas de similaridade entre os padrões, sem nenhum conhecimento sobre as classes de treinamento. Neste trabalho, o problema da segmentação de imagens é visto como um problema de classificação não supervisionada.

Além da avaliaçào de descmpenho dos modelos auto-organizáveis considerados, há ainda, neste trabalho, unia preocupaçào em relação a propostas de melhorias nos resultados de segmentação por meio da incorporação de técnicas de pré-processamento de imagens. Neste caso, o enfoque é dado a técnicas que são capazes de tratar questões referentes à presença de ruídos, suavização de imagens c realce do bordas, de modo que as imagens se tornem mais adequadas ao processo de segmentação. tomando-o mais eficiente.

Os problentas tratados e resolvidos neste trabalho são os seguintes:

- Uina das limitações da rede Fuzzy ART é que esta é sensível a ruídos presentes no conjunto de treinamento. Dessa forma, nó caso deste modelo ser utilizado em um processo de segmentação, a presença de ruídos em imagens pode ser considerada um problema.

- O modelo ICAMM é baseado en una técnica de otimização de gradiente. Portanto, observou-se que o descmpenho dessa técnica é afetado, entre outros fatores, por algumas limitaçôes inerentes a esse tipo de abordagem. Além disso, na tentativa de entender melhor o sen fincionamento. detectou-se que algumas melhorias poderiam ser propostas para tomar o método mais eficiente.

- Não foi encontradla na literatura uma avaliação comparativa dos modelos auto-organizáveis considerados neste trabalho, levando-se cm conta a segmentação de imagens pré-processadas por uma combinação de técnicas para redução de ruídos, suavização de imagens e realce de bordas.

A idéia fundamental deste trabalho baseia-se na proposta de melhorias no desempenho 
de tarefas de segmentação de imagens implementadas por modelos auto-organizáveis. Tais melhorias levam em considcração algumas limitações dos métodos e análises de como estes podem se tornar mais eficientes por meio da utilização de uma metodologia para pré-processamento de imagens.

\subsection{Objetivos}

Considcrando os problemas abordados anteriormentc, este trabalho possui como principais objetivos:

- propor uma metodologia de pré-processamento de imagens que combine técnicas para redução de ruídos, suavização de imagens e realce de bordas;

- propor melhorias a serem adicionadas ao método ICAMM, por meio da incorporação de algumas características de mćtodos de otimização não linear e de modificaçōes referentes a aspectos de implementação e modelagem;

- avaliar como os desempenhos dos métodos auto-organizáveis considerados podem ser afetados pela utilização da metodologia de pré-processamento proposta; e

- realizar um estudo comparativo entre os métodos auto-organizáveis considerados, por meio da análise cxperimental dos desempenhos destes em tarefas de segmentação de imagens, em suas versões originais e pré-processadas.

\subsection{Organização do Trabalho}

Este trabalho está dividido em nove capítulos, sendo esta introdução o primeiro destes.

No Capítulo 2, são apresentados alguns conceitos e definiçōes importantes para a compreensão deste trabalho.

No Capítulo 3, são apresentados alguns métodos clássicos de análise multivariada, tais como o método PCA c técnicas de agrupamento, e modelos de redes neurais que implementam essas técnicas. Dentre estes modelos, está a rede neural Fuzzy ART, que é utilizada nos experimentos com imagens no presente trabalho.

No Capítulo 4, o método ICA é apresentado, juntamente com as suas suposiçōes, restrições e ambiguidades. Além disso, discute-se várias abordagens para realização da ICA e as relaçōes entre as mesmas. 
No Capítulo 5, a metodologia de pré-processamento de imagens proposta neste trabalho é apresentada.

No Capítulo 6. descreve-se o Modelo ICAMM para classificação não supervisionada.

No Capítulo 7, ć proposto o Modelo de Misturas ICA Aperfeiçoado (EICAMM) ${ }^{5}$, resultante de melhorias e incorporaçôes feitas ao modelo ICAMM.

No Capítulo 8 , os resultados expcrimentais deste trabalho são apresentados e discutidos.

Finalmente, no Capítulo 9, são feitas algumas conclusões e considerações sobre trabalhos futuros. As contribuiçoes deste trabalho estão concentradas nos capítulos 5 e 7 .

\footnotetext{
${ }^{5}$ Do original, em ingles, Enhanced ICA Mixture Model.
} 


\section{Capítulo 2}

\section{Definições}

\subsection{Considerações Iniciais}

Neste capítulo, é realizada uma revisão de conceitos importantes da álgebra linear, teoria da probabilidade. estatística e de redes neurais artificiais considerados de fundamental importância para o entendimento do restantc desta tese. É assumido, entretanto, que o leitor possui conhecimento de conceitos básicos de teoria de probabilidade, como por exemplo, os conceitos de eventos elementares e variáveis aleatórias. Uma revisão mais extensa, variando de conceitos básicos aos mais avançados, pode ser encontrada em referências clássicas, como (Papoulis 1991), (Weiss \& Kulikowski 1991), (Grossman 1986).

Deve-se chamar atenção para o fato de que, ao longo de todo este trabalho, literais minúsculas e em itálico (por exemplo, a) são utilizadas para denotar valores escalares, literais minúsculas e em negrito (por exemplo, a) são utilizadas para denotar vetores de valores escalares e literais maiúsculas e em ncgrito (por exemplo, A) são utilizadas para denotar matrizes de valores escalares.

\subsection{Definições e Propriedades de Vetores e Matrizes}

\subsubsection{Norma, Distância Euclidiana e Ortogonalidade de Vetores}

A norma, on comprimcnto, de um vetor $\mathbf{v}=\left(v_{1}, v_{2}, \ldots, v_{n}\right)$ de $\mathbb{R}^{n}$, denotada por $\|\mathbf{v}\|$, é definida como sendo a raiz quadrada de $\mathbf{v} \cdot \mathbf{v}$ :

$$
\|\mathbf{v}\|=\sqrt{\mathbf{v} \cdot \mathbf{v}}=\sqrt{v_{1}^{2}+v_{2}^{2}+\ldots+v_{n}^{2}} .
$$

Sejam $\mathbf{u}=\left(u_{1}, u_{2}, \ldots, u_{n}\right)$ e $\mathbf{v}=\left(v_{1}, v_{2}, \ldots, v_{n}\right)$ vetores de $\mathbb{R}^{n}$. A distância Euclidiana 
entre $\mathbf{u}$ e $\mathbf{v}$ é definida por:

$$
d(\mathbf{u} . \mathbf{v})=\sqrt{\left(u_{1}-v_{1}\right)^{2}+\left(u_{2}-v_{2}\right)^{2}+\ldots+\left(u_{n}-v_{n}\right)^{2}}=\|\mathbf{u}-\mathbf{v}\|
$$

Dois vetores $\mathbf{u}$ : $\mathbf{v}$ de $\mathbb{R}^{n}$ sào considerados ortogonais (ou perpendiculares) se o ângulo entre cstes for igual a 90 graus.

\subsubsection{Matrizes Identidade e Diagonal}

A matriz identidade I é uma matriz $n \times n$, na qual todos elementos da diagonal principal, isto é, $i_{11}, i_{22}, \ldots i_{33}$. sâo iguais a 1 c os elementos fora da diagonal principal, ou seja, $i_{j k}, j \neq k$, são iguais a 0.

Uma matriz A $n \times n$ 6 chamada de diagonal se todos os elementos fora da diagonal principal são iguais a 0 , on seja. $a_{j k}=0$, se $j \neq k$.

\subsubsection{Transposição e Inversão de Matrizes}

A matriz transposta de uma matriz $\mathbf{A} n \times m$, denotada por $\mathbf{A}^{T}$, é a matriz $m \times n$ obtida trocando-se as linhas pelas colunas de $\mathbf{A}$. Em outras palavras, a $i$-ćsima linha de $\mathbf{A}$ é a $i$-ésima coluna de $\mathbf{A}^{T}$ e a j-ésima coluna de $\mathbf{A}$ é a $i$-ésima linha de $\mathbf{A}^{T}$.

Sc para uma dada matriz $\mathbf{A} n \times n$, existe uma matriz $n \times n$, denotada por $\mathbf{A}^{-1}$, tal que

$$
\mathbf{A}^{-1} \mathbf{A}=\mathbf{A A}^{-1}=\mathbf{I}
$$

diz-se que $\mathbf{A}^{-1}$ é a inversa de $\mathbf{A}$.

Uma matriz $\mathbf{A} n \times n$ é dita inversível (ou não singular) se $\mathbf{A}^{-1}$ existe; ou não inversível (singular) se $\mathbf{A}$ não possui inversa.

\subsubsection{Matriz Simétrica e Ortogonal}

Uma matriz simétrica $\mathbf{A}$ ć igual a sua transposta, ou seja, $\mathbf{A}=\mathbf{A}^{T}$. Em outras palavras, para cada par de índices $i$ e $j \cdot a_{i_{j}}=a_{j i}$.

Uma matriz $\mathbf{A} 6$ ortogonal so $\mathbf{A}$ for inversível e $\mathbf{A}^{-1}=\mathbf{A}^{T}$.

\subsubsection{Determinante e Cofator}

Scja $\mathbf{A}$ uma matriz $n \times n$. É possível associar à $\mathbf{A}$ um escalar chamado de determinante de $\mathbf{A}$, denotado por $\operatorname{det}(\mathbf{A})$ e definido, recursivamente a partir de qualquer uma das $i$ linhas da 
matriz $\mathbf{A}$, como sendo a seguinte soma:

$$
\operatorname{det}(\mathbf{A})=a_{i 1} \operatorname{det}\left(\mathbf{A}_{i 1}\right)+a_{i 2} \operatorname{det}\left(\mathbf{A}_{i 2}\right)+\ldots+a_{i n} \operatorname{det}\left(\mathbf{A}_{i n}\right)
$$

ou de uma forma sintética,

$$
\operatorname{det}(\mathbf{A})=\sum_{j=1}^{n} a_{i j} \operatorname{det}\left(\mathbf{A}_{i j}\right),
$$

para cada linha $i=1,2, \ldots, n$ fixada.

Em todas as sitnaçies acima, $\mathbf{A}_{i j}$ significa a matriz de ordem $n-1$, obtida pela exclusão da linha $i$ e da coluna $j$. Uma matriz $\mathbf{A}_{i j}$ obtida dessa forma é denominada de matriz cofatora. Utilizando esse resultado pode-se definir o cofator do elemento $a_{i j}$ de $\mathbf{A}$ por meio da seguinte fórmula:

$$
\operatorname{cof}\left(a_{i j}\right)=(-1)^{i+j} \operatorname{det}\left(\mathbf{A}_{i j}\right)
$$

\subsubsection{Matriz Adjunta}

A matriz adjunta de uma matriz quadrada $\mathbf{A}$, denotada por adj (A), tem a propriedade que:

$\mathbf{A} * \operatorname{adj}(\mathbf{A})=\operatorname{adj}(\mathbf{A}) * \mathbf{A}=\operatorname{det}(\mathbf{A}) * \mathbf{I}$.

Portanto, a inversa de $\mathbf{A}$ pode ser escrita como: $\mathbf{A}^{-1}=(1 / \operatorname{det}(\mathbf{A})) * \operatorname{adj}(\mathbf{A})$.

\subsubsection{Matrizes de Derivadas Parciais}

\section{Matriz Jacobiana}

A matriz Jacobiana é dadia pela seguinte matriz de derivadas parciais:

$$
J=\left[\begin{array}{ccc}
\frac{\partial y_{1}}{\partial x_{1}} & \cdots & \frac{\partial y_{1}}{\partial x_{n}} \\
\vdots & & \vdots \\
\frac{\partial y_{n}}{\partial x_{1}} & \cdots & \frac{\partial y_{n}}{\partial x_{n}}
\end{array}\right]
$$

\section{Matriz Hessiana}

A matriz Hessiana é dada pela seguinte matriz de derivadas parciais: 


$$
J=\left[\begin{array}{ccc}
\frac{\partial^{2} y_{1}}{\partial x_{1}^{2}} & \cdots & \frac{\partial^{2} y_{1}}{\partial x_{n}^{2}} \\
\vdots & & \vdots \\
\frac{\partial^{2} y_{n}}{\partial x_{1}^{2}} & \cdots & \frac{\partial^{2} y_{n}}{\partial x_{n}^{2}}
\end{array}\right]
$$

\subsection{Distribuições e Densidades de Probabilidade}

\subsubsection{Distribuição de uma Variável Aleatória}

A função de distribuição acumulada (fda) $F_{x}$ de uma variável aleatória contínua $x$ no ponto $x=x_{0}$ é definida como a probabilidade de que $x \leq x_{0}$ :

$$
F_{x}\left(x_{0}\right)=P\left(x \leq x_{0}\right)
$$

Fazendo $x_{0}$ variar no intervalo $(-\infty, \infty)$, pode-se definir a fda completa para todos os valores de $x$.

Para variáveis aleatórias contínuas, a fda é uma função contínua, não negativa e não decrescente, cujos valores cncontram-se no intervalo $0 \leq F_{x}(x) \leq 1$. A partir da definição, segue que $F_{x}(-\infty)=0$ e $F_{x}(+\infty)=1$.

Geralmente, uma distribuição de probabilidade é mais caracterizada por sua função de densidade do que por sıa fda. Formalmente, a função de densidade de probabilidade (fdp) de uma variável aleatória contínua $x$ é obtida como a derivada da sua função de distribuição acumulada:

$$
p_{x}\left(x_{0}\right)=\left.\frac{d F_{x}(x)}{d x}\right|_{x=x_{0}} .
$$

Para simplificar a notação. $F_{r}(x)$ é quase sempre denotada por $F(x)$ e $p_{x}(x)$ por $p(x)$, respectivamente.

Para ilustrar esses conceitos. pode-se tomar como exemplo a distribuição de probabilidade normal (ou gaussiana). utilizada $\mathrm{em}$ vários modelos e aplicações. No caso desta distribuição, a função de densidade é dadà por:

$$
p(x)=\frac{1}{\sqrt{2 \pi \sigma^{2}}} \exp \left(-\frac{(x-m)^{2}}{2 \sigma^{2}}\right) .
$$

Aqui, o parâmetro $m$ (néedia) determina o ponto de pico da funçāo de densidade simétrica e $\sigma$ (desvio-padrão) definc a forma da distribuição - se esta é muito dispersa ou se a maior parte da área se concentra na proximidade do pico (ver Figura 2.1). 


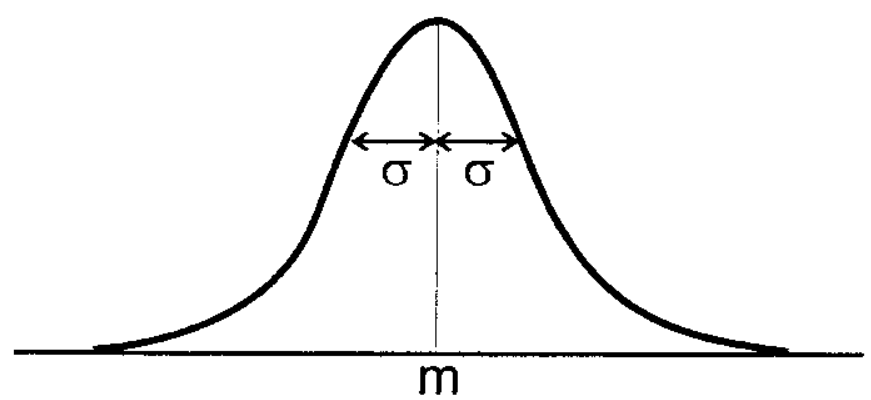

Figura 2.1: Função de densidade de probabilidade de uma distribuição gaussiana com média $m$ e desvio-padrão $\sigma$.

\subsubsection{Distribuição de um Vetor Aleatório}

Assume-se agora que $\mathrm{x}$ é um vetor aleatório $n$-dimensional:

$$
x=\left(x_{1}, x_{2}, \ldots, x_{n}\right)^{T},
$$

onde $T$ denota a transposição de matriz. Neste caso, as componentes $x_{1}, x_{2}, \ldots, x_{n}$ do vetor coluna $\mathrm{x}$ são variáveis aleatórias contínuas e o conceito de distribuição de probabilidade pode ser facilmente generalizado para vetores aleatórios. Em particular, a função de distribuição acumulada de $\mathbf{x}$ é definida por:

$$
F_{\mathbf{x}}\left(\mathbf{x}_{0}\right)=P\left(\mathbf{x} \leq \mathbf{x}_{0}\right),
$$

onde $P($.$) denota a probabilidade do evento em parênteses e \mathbf{x}_{0}$ é alguma instância do vetor aleatório $\mathbf{x}$. A notação $\mathbf{x} \leq \mathbf{x}_{0}$ significa que cada componente do vetor $\mathbf{x}$ é menor ou igual ao seu componente correspondente no vetor aleatório $\mathbf{x}_{0}$.

A função de densidade de probabilidade multivariada $p_{\mathbf{x}}(\mathbf{x})$ de $\mathbf{x}$ é definida como a derivada da função de distribuição acumulada $F_{\mathbf{x}}(\mathbf{x})$ com respeito a todas componentes do vetor aleatório $\mathbf{x}$ :

$$
p_{\mathbf{x}}\left(\mathbf{x}_{0}\right)=\left.\frac{\partial}{\partial x_{1}} \frac{\partial}{\partial x_{2}} \cdots \frac{\partial}{\partial x_{n}} F_{\mathbf{x}}(\mathbf{x})\right|_{\mathbf{x}=\mathbf{x}_{0}} .
$$

\subsubsection{Distribuições Conjunta e Marginal}

Sejam $\mathrm{x}$ e $\mathrm{y}$ dois vetores aleatórios diferentes, com dimensões $n$ e $m$, respectivamente. A função de distribuição conjunta de $\mathbf{x}$ e $\mathbf{y}$ é dada por: 


$$
F_{\mathbf{x}, \mathbf{y}}\left(\mathbf{x}_{0}, \mathbf{y}_{0}\right)=P\left(\mathbf{x} \leq \mathbf{x}_{0}, \mathbf{y} \leq \mathbf{y}_{0}\right)
$$

Neste caso, $\mathbf{x}_{0}$ e $\mathbf{y}_{0}$ são dois vetores constantes que possuem as mesmas dimensões de $\mathbf{x}$ e $\mathbf{y}$, respectivamente, e a Equação (2.15) define a probabilidade conjunta dos eventos $\mathbf{x} \leq \mathbf{x}_{0} \mathrm{e}$ $\mathrm{y} \leq \mathrm{y}_{0}$.

A função de densidade conjunta $p_{\mathbf{x}, \mathbf{y}}(\mathbf{x}, \mathbf{y})$ de $\mathbf{x}$ e $\mathbf{y}$ é definida formalmente diferenciandose a função de distribuição conjunta $F_{\mathbf{x} . \mathbf{y}}(\mathbf{x}, \mathbf{y})$ com respeito a todas as componentes dos vetores aleatórios $\mathbf{x}$ e $\mathbf{y}$. Dessa forma, a relação

$$
F_{\mathbf{x}, \mathbf{y}}\left(\mathbf{x}_{0}, \mathbf{y}_{0}\right)=\int_{-\infty}^{\mathbf{x}_{0}} \int_{-\infty}^{\mathbf{y}_{0}} p_{\mathbf{x}, \mathbf{y}}(\boldsymbol{\xi}, \eta) d \boldsymbol{\eta} d \boldsymbol{\xi}
$$

é satisfeita e o valor dessa integral é igual a 1 quando $\mathbf{x}_{0} \rightarrow \infty$ e $\mathbf{y}_{0} \rightarrow \infty$.

As densidades marginais $p_{\mathbf{x}}(\mathbf{x})$ de $\mathbf{x}$ e $p_{\mathbf{y}}(\mathbf{y})$ de $\mathbf{y}$ são obtidas da seguinte maneira:

$$
\begin{aligned}
& p_{\mathbf{x}}(\mathbf{x})=\int_{-\infty}^{+\infty} p_{\mathbf{x}, \mathbf{y}}(\mathbf{x}, \boldsymbol{\eta}) d \boldsymbol{\eta} \\
& p_{\mathbf{y}}(\mathbf{y})=\int_{-\infty}^{+\infty} p_{\mathbf{x}, \mathbf{y}}(\boldsymbol{\xi}, \mathbf{y}) d \boldsymbol{\xi} .
\end{aligned}
$$

\subsection{Esperança Matemática e Momentos}

\subsubsection{Definições e Propriedades Gerais}

Na prática a função de densidade de probabilidade exata de uma variável aleatória escalar ou vetorial é quase sempre desconhecida. Todavia, pode-se usar, ao invés desta função, esperanças matcmáticas de algumas funções da variável em questão para efetuar análises c processamentos. A grande vantagem das esperanças matemáticas é que estas podem ser estimadas diretamente a partir dos dados observados, apesar de serem definidas formalmente em termos da função de densidade.

Neste caso, pode-se denotar por $\mathbf{g}(\mathbf{x})$ qualquer quantidade derivada a partir do vetor aleatório $\mathbf{x}$. A quantidade $\mathbf{g}(\mathbf{x})$ pode ser escalar, vetorial, ou até mesmo matricial. A esperança matemática de $\mathbf{g}(\mathbf{x})$ é denotada por $E\{\mathbf{g}(\mathbf{x})\}$ e definida por:

$$
E\{\mathrm{~g}(\mathbf{x})\}=\int_{-\infty}^{+\infty} g(\mathbf{x}) p_{\mathbf{x}}(\mathbf{x}) d \mathbf{x}
$$

Neste caso, o operador de integral é aplicado separadamente a cada componente do vetor ou 
elemento da matriz, resultando crn um outro vetor ou matriz do mesmo tamanho.

\subsubsection{Vetor de Média e Matriz de Correlação}

Momentos de um vetor aleatório $\mathbf{x}$ são esperanças matemáticas típicas usadas para caracterizar esse vetor. Tais valores são obtidos quando $\mathbf{g}(\mathbf{x})$ consiste de produtos das componentes de $\mathbf{x}$. Em particular, o primeiro momento de uma variável aleatória $\mathbf{x}$ é chamado de vetor de média $\mathbf{m}_{\mathbf{x}}$ de $\mathbf{x}$. Esse momento é definido como o valor esperado de $\mathbf{x}$, que é expressa por meio da seguinte equaçāo:

$$
\mathbf{m}_{\mathbf{x}}=E\{\mathbf{x}\}=\int_{-\infty}^{+\infty} \mathbf{x} p_{\mathbf{x}}(\mathbf{x}) d \mathbf{x}
$$

Cada componente $m_{r_{2}}$ do vetor $m_{\mathrm{x}}$ é dada por:

$$
\mathbf{m}_{i,}=E\left\{x_{i}\right\}=\int_{-\infty}^{+\infty} x_{i} p_{\mathbf{x}}(\mathbf{x}) d \mathbf{x}=\int_{-\infty}^{+\infty} x_{i} p_{x_{i}}\left(x_{i}\right) d x_{i},
$$

onde $p_{x_{i}}\left(x_{i}\right)$ é a densidade marginal da $i$-ésima componente de $\mathbf{x}$.

Outro conjunto de momentos importantes consiste nas correlações entre pares de componentes de $\mathbf{x}$. A corrclaçăo $r_{i j}$ cntre a $i$-ésima e a $j$-ésima componente de $\mathbf{x}$ é dada pelo momento de segunda ordem:

$$
r_{i j}=E\left\{x_{i} x_{j}\right\}=\int_{-\infty}^{+\infty} x_{i} x_{j} p_{\mathbf{x}}(\mathbf{x}) d \mathbf{x}=\int_{-\infty}^{+\infty} \int_{-\infty}^{+\infty} x_{i} x_{j} p_{x_{2}} p_{x_{j}}\left(x_{i}, x_{j}\right) d x_{j} d x_{i}
$$

A matriz de correlação $n \times n$ :

$$
\mathbf{R}_{\mathbf{x}}=E\left\{\mathbf{x} \mathbf{x}^{T}\right\}
$$

do vetor $\mathbf{x}$ representa todas as correlaçōes entre as componentes do vetor, sendo $r_{i j}$ o elemento na linha $i$ e coluna $j$ da matriz $\mathbf{R}_{\mathbf{x}}$.

A matriz de correlação $\mathbf{R}_{\mathrm{x}}$ possui as seguintes propriedades importantes:

1. É uma matriz simétrica, ou seja, $\mathbf{R}_{\mathbf{x}}=\mathbf{R}_{\mathbf{x}}^{T}$.

2. É uma matriz positiva semi-definida:

$$
\mathbf{a}^{T} \mathbf{R}_{\mathbf{x}} \mathbf{a}>0
$$


para todos os vetores a $n$-dimensionais.

3. Todos os alto-valores de $\mathbf{R}_{\mathbf{x}}$ são reais e não negativos (positivos se $\mathbf{R}_{\mathbf{x}}$ for positiva definida).

\subsubsection{Matriz de Covariância}

A matriz de covariância $C_{\mathbf{x}}$ de $\mathbf{x}$ é dada por:

$$
\mathbf{C}_{\mathbf{x}}=E\left\{\left(\mathbf{x}-\mathbf{m}_{\mathbf{x}}\right)\left(\mathbf{x}-\mathbf{m}_{\mathbf{x}}\right)^{T}\right\}
$$

Os elementos

$$
\mathbf{c}_{i j}=E\left\{\left(x_{i}-m_{i}\right)\left(x_{j}-m_{j}\right)\right\}
$$

da matrix $n \times n \mathbf{C}_{\mathbf{x}}$ são chamados de covariâncias, sendo estas correspondentes às correlações $r_{i j}$, quando o vetor aleatório $\mathbf{x}$ tiver sido subtraído do seu vetor de média correspondente.

A matriz de covariancia $\mathbf{C}_{\mathbf{x}}$ apresenta as mesmas propriedades da matriz de correlação $R_{\mathbf{x}}$. Sc o vetor de média $\mathrm{m}_{\mathrm{x}}=\mathbf{0}$, as matrizes de covariância e de correlação são as mesmas. Se for necessário, os dados podem ser facilmente transformados de modo a possuírem um vetor de médias igual à zero, subtraindo destes o vetor de média (estimado) dos vetores de dados, em uma etapa de pré-processamento. Essa prática é considerada comum nas técnicas PCA e ICA.

Para uma variável aleatória unidimensional $x$, o vetor de médias fica reduzido ao valor médio $m_{x}=E\{x\}$. a matriz de correlaçāo reduz-se ao segundo momento $E\left\{x^{2}\right\}$ e a matriz de covariância à variância de $x$ :

$$
\sigma_{x}^{2}=E\left\{\left(x-m_{x}\right)^{2}\right\}
$$

onde $\sigma_{x}$ corresponde ao desvio-padrão de $x$.

O operador de esperança pode ser estendido para funções $\mathbf{g}(\mathbf{x}, \mathbf{y})$ de dois vetores aleatórios $\mathbf{x}$ e $\mathbf{y}$, em termos da sua densidade conjunta:

$$
E\{\mathbf{g}(\mathbf{x}, \mathbf{y})\}=\int_{-\infty}^{+\infty} \int_{-\infty}^{+\infty} \mathbf{g}(\mathbf{x}, \mathbf{y}) p_{\mathbf{x}, \mathbf{y}}(\mathbf{x}, \mathbf{y}) d \mathbf{x} d \mathbf{y} .
$$

Neste caso, as integrais são calculadas sobre todas as componentes de $\mathbf{x}$ e $\mathbf{y}$.

Dentre os valores cspcrados conjuntos mais utilizados, destacam-se a matriz de correlaçâao cruzada:

$$
\mathbf{R}_{\mathbf{x y}}=E\left\{\mathbf{x y}^{T}\right\}
$$


e a matriz de covariância cruzada:

$$
\mathbf{C}_{\mathbf{x y}}=E\left\{\left(\mathbf{x}-\mathbf{m}_{\mathbf{x}}\right)\left(\mathbf{y}-\mathbf{m}_{\mathbf{y}}\right)^{T}\right\}
$$

É importante notar que as dimensões dos vetores $x$ e $y$ podem ser diferentes. Consequentemente, as matrizes de covariância e correlação cruzadas não são necessariamente matrizes quadradas e, em geral, não são simétricas. Entretanto, a partir das definições dessas duas matrizes, segue que:

$$
\begin{aligned}
\mathbf{R}_{\mathbf{x y}} & =\mathbf{R}_{\mathbf{y x}}^{T} \\
\mathbf{C}_{\mathbf{x y}} & =\mathbf{C}_{\mathbf{y} \mathbf{x}}^{T} .
\end{aligned}
$$

Se os vetores de médias de $\mathbf{x}$ e $\mathbf{y}$ forem iguais à zero, as matrizes de correlação e covariância cruzadas são iguais. De fato, quarritidades de correlação e covariância medem a dependência entre as variáveis aleatórias usando as suas estatísticas de segunda ordem.

\subsubsection{Estimativas de Esperanças}

Geralmente a densidade de probabilidade de um vetor aleatório $\mathbf{x}$ não é conhecida mas quase sempre existe un conjunto disponivel de $K$ amostras $\mathbf{x}_{1}, \mathbf{x}_{2}, \ldots, \mathbf{x}_{K}$ de $\mathbf{x}$. Usando essas amostras, a esperança na Equação (2.19) pode ser estimada, tomando-se a média sobre toda as amostras, usando a seguinte fórmula:

$$
E\{\mathrm{~g}(\mathrm{x})\} \approx \frac{1}{K} \sum_{j=1}^{K} \mathrm{~g}\left(\mathbf{x}_{j}\right) .
$$

Por excmjlo, aplicando a Equação (2.33), obtém-se para o vetor de médias $\mathbf{m}_{\mathbf{x}}$ de $\mathbf{x}$ o seu estimador padrão. a média amostral:

$$
\hat{\mathbf{m}}_{\mathbf{x}}=\frac{1}{K} \sum_{j=1}^{K} \mathbf{x}_{j}
$$

onde o símbolo chapéu sobre m é uma notação padrāo para um estimador de uma quantidade. Ainda, para a matriz de corrclaçào de um vetor alcatória, a estimação resulta em:

$$
\hat{\mathbf{R}}_{\mathbf{x}}=\frac{1}{K} \sum_{i=1}^{n} \sum_{j=1}^{n} x_{i} x_{j}^{T}
$$


De maneira similar. ao invés da densidade conjunta $p_{\mathbf{x}, \mathbf{y}}$ dos vetores aleatórios $\mathbf{x}$ e $\mathbf{y}$, podese usar $K$ pares amostrais $\left(\mathbf{x}_{1}, \mathbf{y}_{1}\right),\left(\mathbf{x}_{2}, \mathbf{y}_{2}\right), \ldots,\left(\mathbf{x}_{K}, \mathbf{y}_{K}\right)$ para estimar a esperança da Equação (2.28) por meio de:

$$
E\{\mathrm{~g}(\mathrm{x}, \mathrm{y})\} \approx \frac{1}{K} \sum_{j=1}^{K} \mathrm{~g}\left(\mathbf{x}_{j}, \mathbf{y}_{j}\right)
$$

Por exemplo, para a matriz de correlação cruzada, a estimação resulta na seguinte fórmula:

$$
\hat{\mathbf{R}}_{\mathbf{x y}}=\frac{1}{K} \sum_{j=1}^{K} \mathbf{x}_{j} \mathbf{y}_{j}^{T}
$$

\subsection{Não Correlação e Branqueamento}

Dois vetores aleatórios $\mathbf{x}$ e $\mathbf{y}$ são não correlacionados se a correspondente matriz de covariância cruzada $\mathbf{C}_{\mathbf{x y}}$ for uma matriz com todos elementos iguais à zero:

$$
\mathbf{C}_{\mathbf{x y}}=E\left\{\left(\mathbf{x}-\mathbf{m}_{\mathbf{x}}\right)\left(\mathbf{y}-\mathbf{m}_{\mathbf{y}}\right)\right\}=\mathbf{0} .
$$

Isso é equivalente à condição:

$$
\mathrm{R}_{\mathbf{x y}}=E\left\{\mathrm{xy}^{T}\right\}=E\{\mathbf{x}\}\left\{\mathrm{y}^{T}\right\}=\mathbf{m}_{\mathbf{x}} \mathbf{m}_{\mathbf{y}}^{T} .
$$

Em especial. duas variáveis aleatórias unidimensionais diferentes $x$ e $y$ são não correlacionadas se a covariância entre estas duas variáveis $c_{x y}$ for igual à zero:

$$
c_{x y}=E\left\{\left(x-m_{x}\right)\left(y-m_{y}\right)\right\}=0
$$

on de forma equivalente.

$$
r_{x y}=E\{x y\}=E\{x\}\{y\}=m_{x} m_{y} .
$$

Assim como no caso de variáveis multivariadas com média zero, no caso univariado, covariância zero é equivalente à correlação zcro.

Um outro caso especial importante diz respcito às correlações entre as componentes de um único vetor aleatório $\mathbf{x}$. dadas pela matriz de covariância $\mathbf{C}_{\mathbf{x}}$, definida na Equação (2.25). Nesse caso, a condição equivalente à Equaçāo (2.38) nunca pode ser satisfeita, uma vez que cada componente do vetor $\mathrm{x}$ é perfeitamente correlacionada com ela mesma. A maneira mais 
próxima de atingir essa condição é quando as diferentes componentes de $\mathbf{x}$ são mutuamente não correlacionadas, levando à seguinte condição:

$$
\mathbf{C}_{\mathbf{x}}=E\left\{\left(\mathbf{x}-\mathbf{m}_{\mathbf{x}}\right)\left(\mathbf{x}-\mathbf{m}_{\mathbf{x}}\right)^{T}=\mathbf{D}\right.
$$

Nesse caso, $\mathbf{D}$ é a matriz diagonal $n \times n$ :

$$
\mathbf{D}=\operatorname{diag}\left(c_{11}, c_{22}, \ldots, c_{n n}\right)=\operatorname{diag}\left(\sigma_{x 1}^{2}, \sigma_{x 2}^{2}, \ldots, \sigma_{x n}^{2}\right),
$$

cujos $n$ elementos diagonais são as variâncias $\sigma_{x i}^{2}=E\left\{\left(x_{i}-m_{x_{i}}\right)^{2}\right\}=c_{i i}$ das componentes $x_{i}$ de $\mathbf{x}$.

Em particular, vetores aleatórios com média zero e matriz de covariância (e consequentemente de correlação) igual à matriz identidade, ou possivelmente multiplicada por uma variância $\sigma^{2}$ constante, são chamados de brancos. Esse nome deve-se à semelhança com a definição de ruídos brancos, que sāo variáveis aleatórias não correlacionadas, com variâncias iguais a um. Dessa forma, vetores aleatórios brancos satisfazem as seguintes condiçōes:

$$
\mathbf{m}_{\mathbf{x}}=\mathbf{0}
$$

$\mathrm{c}$

$$
\mathrm{R}_{\mathrm{x}}=\mathrm{C}_{\mathrm{x}}=\mathrm{I}
$$

onde I é a matriz identidade $n \times n$.

\subsection{Independência Estatística}

Independência estatística é um conceito importante que constitui a base para a técnica ICA. Para simplificar o entendimento deste conceito, pode-se considerar primeiramente o caso de duas variáveis escalares (unidimensionais) $x$ e $y$. A variável aleatória $x$ é independente de $y$ se for conhecido que o valor de $y$ não fornece nenhuma informação sobre o valor de $x$. Por exemplo, $x$ e $y$ podem ser us valores de saída de dois eventos que não têm nenhuma relação um com o o outro, ou sinais alcatórios originários de dois processos físicos completamente distintos. Como exemplo dessas variáveis aleatórias, pode-se citar o valor de um dado e de uma moeda lançados, on um sinal do fala e unn ruído de fundo ocasionado por um sistema de ventilação $\mathrm{cm}$ um determinado instante de tempo. 
Matematicamente, independência estatística é definida em termos de densidades de probabilidade. As variávcis alcatórias $x$ e $y$ são consideradas independentes se, e somente se:

$$
p_{x, y}(x, y)=p_{x}(x) p_{y}(y)
$$

Colocando cm palavras. a densidade conjunta $p_{x, y}(x, y)$ de $x$ e $y$ tem que ser fatorada no produto das suas densidades marginais $p_{x}(x)$ e $p_{y}(y)$.

Variáveis aleatórias independentes satisfazem a seguinte propriedade básica:

$$
E\{g(x) h(y)\}=E\{g(x)\} E\{h(y)\}
$$

onde $g(x)$ e $h(y)$ são quaisquer funções integráveis de $x$ e $y$, respectivamente. Essa propriedade revela que o conccito de independência estatística é muito mais forte do que o conceito de não correlação. A Equação (2.41), que define o conceito de não correlação, é obtida a partir da propriedade de independencia da Equação (2.47) como um caso especial, quando $g(x)$ e $h(y)$ são funções lineares. e que levam em conta somente estatísticas de segunda ordem (correlações ou covariâncias). Entretanto, sc as variáveis alcatórias apresentarem distribuições Gaussianas, os conceitos de independència e não correlação tornam-se equivalentes. Essa é uma propriedade muito especial das distribuiçôes Gaussianas que será discutida na Seção (2.8).

A definição de independência na Equação (2.46) pode ser generalizada de forma natural para mais de duas variáveis ou para vetores aleatórios. Sejam $\mathbf{x}, \mathbf{y}, \mathbf{z} \ldots$, vetores aleatórios que podem, em geral, ter dimensôes diferentes. A condição de independência para $\mathbf{x}, \mathbf{y}, \mathbf{z}, \ldots, \dot{c}$, portanto:

$$
p_{\mathbf{x}, \mathbf{y}, \mathbf{z} \ldots}(\mathbf{x}, \mathbf{y}, \mathbf{z} \ldots)=p_{\mathbf{x}}(\mathbf{x}) p_{\mathbf{y}}(\mathbf{y}) p_{\mathbf{z}}(\mathbf{z}) \ldots
$$

e a propriedade básica da Equação (2.47) pode ser generalizada para:

$$
E\left\{\mathbf{g}_{\mathbf{x}}(\mathbf{x}) \mathbf{g}_{\mathbf{y}}(\mathbf{y}) \mathbf{g}_{\mathbf{z}}(\mathbf{z}) \ldots\right\}=E\left\{\mathbf{g}_{\mathbf{x}}(\mathbf{x})\right\} E\left\{\mathbf{g}_{\mathbf{y}}(\mathbf{y})\right\} E\left\{\mathbf{g}_{\mathbf{z}}(\mathbf{z})\right\} \ldots
$$

onde $\mathbf{g}_{\mathbf{x}}(\mathbf{x}), \mathbf{g}_{\mathbf{y}}(\mathbf{y})$ e $\mathbf{g}_{\mathbf{z}}(\mathbf{z})$ são funções arbitrárias das variáveis aleatórias $\mathbf{x}, \mathbf{y}$ e $\mathbf{z}$ para as quais as esperanģas da Equaçĩo (2.49) existem.

A definição geral da Equação (2.48) dá origem a uma generalização da noção padrão de independência estatística. As componentes do vetor aleatório $\mathrm{x}$ são, por si só, variáveis aleatórias escalares. o mesmo acontecendo para as componentes de y e $\mathbf{z}$. Claramente, as componentes de $\mathrm{x}$ podem ses mutuamente dependentes, apesar de serem independentes com 
respeito às componentes dos outros vetores aleatórios. Ainda nessa situação, a Equação (2.48) ć satisfeita. Argumentos similares aplicam-se aos vetores $\mathbf{y}$ e z.

\subsection{Densidades Condicionais e Regra de Bayes}

Além das densidades de probabilidade, densidades conjuntas e densidades marginais, uma outra classe importante de funçōes de densidade de probabilidade consiste nas densidades condicionais. Estas densidades surgem como resposta à seguinte questão "Qual é a densidade de probabilidade de um vetor aleatório $\mathbf{x}$ dado que um outro vetor aleatório $\mathbf{y}$ tem um valor fixo $\mathbf{y}_{0} ? "$

Assumindo que a densidade conjunta $p_{\mathbf{x}, \mathbf{y}}(\mathbf{x}, \mathbf{y})$ de $\mathbf{x}$ e $\mathbf{y}$ e as suas respectivas probabilidades marginais existem. a densidade de probabilidade condicional de $\mathbf{x}$ dado $\mathbf{y}$ é dada por:

$$
p_{\mathbf{x} \mid \mathbf{y}}(\mathbf{x} \mid \mathbf{y})=\frac{p_{\mathbf{x}, \mathbf{y}}(\mathbf{x}, \mathbf{y})}{p_{\mathbf{y}}(\mathbf{y})}
$$

Essa definição pode ser interpretada da seguinte forma: assumindo que o vetor aleatório $\mathrm{y}$ encontra-se na região $\mathrm{y}_{0}<\mathbf{y} \leq \mathrm{y}_{0}+\Delta \mathbf{y}$, a probabilidade de que $\mathrm{x}$ encontre-se na região $\mathbf{x}_{0}<\mathbf{x} \leq \mathbf{x}_{0}+\Delta \mathrm{x}$ é $p_{\mathbf{x} \mid \mathbf{y}}(\mathbf{x} \mid \mathbf{y}) \Delta \mathbf{x}$. Neste caso, $\mathbf{x}_{0}$ e $\mathbf{y}_{0}$ são dois vetores constantes e $\Delta \mathbf{x}$ e $\Delta \mathbf{y}$ são considerados dois vetores relativamente pequenos. De maneira similar:

$$
p_{\mathbf{y} \mid \mathbf{x}}(\mathbf{y} \mid \mathbf{x})=\frac{p_{\mathbf{x}, \mathbf{y}}(\mathbf{x}, \mathbf{y})}{p_{\mathbf{x}}(\mathbf{x})} .
$$

Se os vetores aleatórios $\mathbf{x}_{0}$ e $\mathbf{y}_{0}$ forem estatisticamente independentes, a densidade condicional $p_{\mathbf{x} \mid \mathbf{y}}(\mathbf{x} \mid \mathbf{y})$ é equivalente à densidade incondicional $p_{\mathbf{x}}(\mathbf{x})$ de $\mathbf{x}$, uma vez que $\mathbf{x}$ não depende absolutamente de $\mathbf{y}$ e; de maneira similar, $p_{\mathbf{y} \mid \mathbf{x}}(\mathbf{y} \mid \mathbf{x})=p_{\mathbf{y}}(\mathbf{y})$. Dessa forma. as Equações (2.50) e (2.51) poderiam ser escritas na forma:

$$
p_{\mathbf{x}, \mathbf{y}}(\mathbf{x}, \mathbf{y})=p_{\mathbf{x}}(\mathbf{x}) p_{\mathbf{y}}(\mathbf{y})
$$

que é exatamente a definição de independência para os vetores aleatórios $\mathbf{x}$ e $\mathbf{y}$.

No caso geral, é possivel escrever, a partir das Equações (2.50) e (2.51) duas expressões diferentes para a densidade conjunta de $\mathbf{x}$ e $\mathbf{y}$ :

$$
p_{\mathbf{x}, \mathbf{y}}(\mathbf{x}, \mathbf{y})=p_{\mathbf{y} \mid \mathbf{x}}(\mathbf{y} \mid \mathbf{x}) p_{\mathbf{x}}(\mathbf{x})=p_{\mathbf{x} \mid \mathbf{y}}(\mathbf{x} \mid \mathbf{y}) p_{\mathbf{y}}(\mathbf{y})
$$

A partir dessa fórmula, pode-se encontrar, por exemplo, uma solução para a densidade 
de $\mathbf{y}$ condicionada à $\mathbf{x}$ :

$$
p_{\mathbf{y} \mid \mathbf{x}}(\mathbf{y} \mid \mathbf{x})=\frac{p_{\mathbf{x} \mid \mathbf{y}}(\mathbf{x} \mid \mathbf{y}) p_{\mathbf{y}}(\mathbf{y})}{p_{\mathbf{x}}(\mathbf{x})}
$$

A Equação (2.54) ć chamada de Regra de Bayes. Essa regra é especialmente importante para solução de problemas de estimação estatística, nos quais $p_{\mathbf{x} \mid \mathbf{y}}(\mathbf{x} \mid \mathbf{y})$ é a densidade condicional do vetor de observaçōes $\mathbf{x}$, com $\mathbf{y}$ denotando o vetor de parâmetros aleatórios desconhecidos. A regra de Bayes da Equação (2.54) permite a computação da densidade a posteriori $p_{\mathbf{y} \mid \mathbf{x}}(\mathbf{y} \mid \mathbf{x})$ dos parâmetros $\mathbf{y}$, dado um vetor de observações $\mathbf{x}$ específico e assumindo (ou conhecendo) a distribuição a priori $p_{\mathbf{y}}(\mathbf{y})$ dos parâmetros aleatórios $\mathbf{y}$.

\subsection{A Densidade Gaussiana Multivariada}

A densidade nomal ou gaussiana multivariada possui propriedades especiais que a tornam única entre as funçōes de densidade de probabilidade.

Um vetor $n$-dimensional $\mathrm{x}$ é dito gaussiano se a sua função de densidade de probabilidade possui a seguinte forma:

$$
p_{\mathbf{x}}(\mathbf{x})=\frac{1}{(2 \pi)^{\frac{n}{2}}\left(\operatorname{det} \mathbf{C}_{\mathbf{x}}\right)^{\frac{1}{2}}} \exp \left(\frac{1}{2}\left(\mathbf{x}-\mathbf{m}_{\mathbf{x}}\right)^{T} \mathbf{C}_{\mathbf{x}}^{-1}\left(\mathbf{x}-\mathbf{m}_{\mathbf{x}}\right)\right)
$$

lembrando que, $n$ ć a dimınsão de $\mathbf{x}, \mathbf{m}_{\mathbf{x}}$ ć a média desse vetor $\mathbf{c}_{\mathbf{x}} \mathbf{C}_{\mathbf{x}}$ é matriz de covariância de $\mathbf{x}$. A notação det $\mathbf{C}_{\mathbf{x}}$ é usada para representar o determinante da matriz $\mathbf{C}_{\mathbf{x}}$. É importante notar que a matriz de covariância $\mathrm{C}_{\mathbf{x}}$ é assumida como sendo positiva definida, o que implica que a sua inversa existe.

Pode-se mostrar que, para a densidade da Equação (2.55):

$$
\begin{gathered}
E\{\mathbf{x}\}=\mathbf{m}_{\mathbf{x}} \\
E\left\{\left(\mathbf{x}-\mathbf{m}_{\mathbf{x}}\right)\left(\mathbf{x}-\mathbf{m}_{\mathbf{x}}\right)^{T}\right\}=\mathbf{C}_{\mathbf{x}} .
\end{gathered}
$$

\subsubsection{Propriedades da Densidade Gaussiana}

Aqui são listadas as propricdades mais importantes da densidade Gaussiana multivariada. As provas matemáticas de tais propriedades podem ser encontras em (Papoulis 1991). 
- Somente são necessárias estatísticas de primeira e segunda ordem. O conhecimento do vetor de médias $\mathbf{m}_{\mathbf{x}}$ e da matriz de covariância $\mathbf{C}_{\mathbf{x}}$ de $\mathbf{x}$ é suficiente para definir completamente a densidade gaussiana. Uma consequência importante desse fato e da forma da densidade gaussiana é que métodos de processamento lineares baseados em informaçōes estatísticas de primeira e segunda ordem têm geralmente um desempenho ótimo em situaçoes que lidam com dados gaussianos.

- Transformações lineares são gaussianas. Seja $\mathrm{x}$ um vetor aleatório gaussiano e $\mathbf{y}=$ Ax uma transformação linear de $\mathbf{x}$. Então, $\mathbf{y}$ também é um vetor aleatório Gaussiano com vetor de médias $\mathbf{m}_{\mathbf{y}}=\mathbf{A} \mathbf{m}_{\mathbf{x}}$ e matriz de covariância $\mathbf{C}_{\mathbf{y}}=\mathbf{A} \mathbf{C}_{\mathbf{x}} \mathbf{A}^{T}$

- Não correlação. Variáveis aleatórias gaussianas não correlacionadas também são independentes.

\subsection{Outros Conceitos Importantes}

Nesta seção. são definidos alguns conceitos e procedimentos importantes utilizados na aplicação e avaliaçào de mítodos auto-organizáveis.

\section{Padronização de Dados}

Antes de proceder con algumas análises, como a ICA e PCA, as variáveis $u_{i}$ dos dados originais devem ser padronizadas, de modo a ficarem com média igual a zero e variância igual a um. Isso é feito de acordo com a seguinte fórmula:

$$
u_{i}=\frac{u_{i}-m_{u_{i}}}{\sigma_{u_{i}}}
$$

\section{Normalização de Vetores}

Uma prática muito comum em algoritmos de aprendizagem é a normalização de vetores u para fazer com que scus valores fiquem no intervalo $(0,1)$. Para isto, a seguinte equação é utilizada:

$$
\mathbf{u}=\frac{\mathbf{u}}{\|\mathbf{u}\|}=\frac{\mathbf{u}}{\sqrt{\sum_{i=1}^{m} u_{i}^{2}}}
$$




\section{Matriz de Confusão}

No processo de avaliação do desempenho de um método para classificação, a matriz de confusão é uma matriz que indica quantos elementos, de cada classe, foram agrupados corretamente nas classes formadas pelo modelo (Peper, Zhang \& Noda 1993).

\section{Ciclo de Treinamento}

No processo de treinamento de uma rede neural, um ciclo significa uma apresentação completa do conjunto de padrões.

\section{Taxa de Erro Verdadeiro}

A taxa de erro verdadeiro de um classificador é estatisticamente definida como a taxa de exro do modelo de classificação en um número assintoticamente grande de novos casos que converge, no limite, para verdadeira distribuição da população (Weiss \& Kulikowski 1991).

Uma taxa de crro cmpírica pode ser definida como a razão entre o número de erros cometido pelo modelo do classificação e o número de casos apresentados ao modelo após o treinamento, da seguinte forma:

$$
\text { taxa de erro }=\frac{\text { número de erros }}{\text { número de casos }}
$$

Se um número ilimitado de casos estivesse disponivel, a taxa de erro verdadeiro poderia ser diretamente calculada à medida que o tamanho da amostra aproxima-se do infinito. Entretanto, no mundo real, o tamanho da amostra de dados disponível é sempre finito, e na maioria das vezcs, é relativamente pequeno. A questão, portanto, é se é possível extrapolar das taxas de erro empíricas, calculadas para resultados de uma amostra pequena, para a taxa de erro verdadeiro. Para resolver esse problema, foram desenvolvidas várias técnicas para apresentar padrões a um classificador com o objetivo de conseguir melhores estimativas para as taxas de erro verdadeiro. Dentre esses métodos, a técnica de Validação Cruzada (Cross-Validation) é una das mais utilizadas para estimar o erro verdadeiro de um algoritmo de classificação e será brevemente descrita a seguir.

\section{Validação Cruzada (Cross-Validation)}

O método de validação cruzada consiste em dividir o conjunto de dados em $k$ partições (folds) de tamanhos (aproximadamente) iguais. O método de classificação é executado (treinado) $k$ vezes, sendo que a cada vez cada um dos subconjuntos é deixado de fora do conjunto de 
treinamento, para scr utilizado como conjunto de teste, a partir do que é medido o erro de classificação para aquela execução (treinamento).

\subsection{Considerações Finais}

Neste capítulo, foram apresentados alguns conceitos, definiçōes e propriedades importantes da álgebra lincar. teoria da probabilidade, estatística e de redes neurais artificiais que servirão como basc para compreensão dos métodos apresentados nos capítulos que seguem, assim como para o entendimento da metodologia aplicada na análise de resultados experimentais apresentada no Capítulo 8. 


\section{Capítulo 3}

\section{Métodos de Análise Multivariada e Redes Neurais Artificiais}

\subsection{Considerações Iniciais}

Neste capítulo, são apresentadas duas técnicas estatísticas de análise multivariada consideradas importantes para compreensào de aspectos fundamentais relacionados a outros métodos e análises de resultados apresentados no decorrer desta tese. Além disso, dois modelos de redes neurais auto-organizáveis que implementam estas técnicas também sâo apresentados.

A primeira técnica de análise multivariada a ser descrita é a Análise de Componentes Principais (PCA). que é talvez a mais antiga e bem conhecida técnica da análise multivariada (Johnson \& Wichern 1998). tendo sido descrita primeiramente por Karl Pearson em 1901 (Pcarson 1901). Ele acreditava, aparentemente, que essa era a solução correta para alguns problemas de biomedicina da êpoca. apesar de não chegar a propor um método prático para calcular a análise para mais de duas variáveis. Uma descrição de métodos computacionais vcio mais tarde com Hotelling (Hotclling 1933). que utilizou a PCA em um trabalho realizado em psicometria. Mesmo assim, os cálculos eram extremamente exaustivos quando o problema envolvia muitas variáveis, já que tinharn cue scr fcitos manualmente. Somente depois do advento dos computadores eletrônicos, essa técnica tornou-se amplamente utilizada.

O propósito da PCA é identificar a estrutura de dependência existente em um conjunto de observaçōes multivariadas, con o objetivo de obter uma representação compacta desses dados que conserve as principais características presentes nas observaçōes originais. O entendimento da PCA é de suma importância para o presente trabalho, uma vez que o método da Análise de Componentes Independentes (ICA) pode ser visto como uma generalização deste método. 
A técnica de agrupamento (clustering) é o outro método de análise multivariada aprescntado neste capítulo, uma vez que o método $k$-médias, bascado nessa técnica, é utilizado en comparações nas análises de resultados experimentais realizadas neste trabalho.

Uma outra ahordagem que pode ser utilizada para implementar as técnicas de análise multivariada apresentadas é aquela que utiliza modelos de Redes Neurais Artificiais (RNA). A rede neural PCA Adaptativa é um modelo auto-organizável que implementa a técnica PCA. Este modelo é apresentado neste capítulo, juntamente com alguns resultados da aplicaçâo do mesmo para compressão de imagens médicas.

A técnica de agrupamento pode ser implementada por um modelo neural da família ART, que combina fundamentos da teoria de conjuntos nebulosos com a teoria ART, sendo por isso, denominado de rede nellral Fuzzy ART. A teoria ART é descrita neste capítulo, juntamente com o modelo neural Fuzzy ART. que é un dos métodos auto-organizáveis utilizados nos expcrimentos de segmentaçăo de imagens deste traballo.

\subsection{Métodos de Análise Multivariada}

Pude-se definir Análise Multivariada como o ramo da estatística responsável por examinar como variávcis dependentes relacionam-se dentro de um determinado conjunto de dados. Para isso, deve ser considerado um conjunto de $n$ observaçôs, cada qual contendo valores para $p$ variáveis (Johnson \&: Wichern 1998).

Dentre os objetivos da análise multivariada, pode-se destacar:

- Redução de dados. Com a finalidade de tornar a interpretação dos dados mais fácil, estes sâo reduzidos para a forma mais simples possível. sem sacrificar informações rclevantes. Isso pode ser feito. por exemplo, transformando um conjunto de variáveis interdependentes em variáveis independentes. ou reduzindo a dimensão do conjunto de dados em questão.

- Agrupamento dos dados. Grupos de variáveis ou objetos "similares" são criados, baseandose em medidas de características.

- Investigação de dependência cntre variáveis. É estabelecida a natureza do relacionamento entre as variáveis, jsto é. se as variáveis são mutuamente independentes ou se existem uma ou mais variáveis que dependem de outras.

- Previsão. Os relacionamentos entre as variáveis devem ser determinados com a finalidade de prever os valores de uma ou mais variáveis de interesse. 


\subsubsection{Análise de Componentes Principais (PCA)}

Análise de Componentes Principais é um dos métodos estatísticos multivariados mais simples, consistindo de: dadas $p$ variáveis $\mathbf{x}_{1}, \mathbf{x}_{2}, \ldots, \mathbf{x}_{p}$, deseja-se achar combinações lineares dessas, para produzir índicess $\mathbf{z}_{1}, \mathbf{z}_{2}, \ldots, \mathbf{z}_{p}$, que sejam nāo correlacionados.

A falta de correlação é una propricdade útil pois significa que os índices estāo medindo diferentes "dimensões" dos dados. É importante ressaltar que os índices são ordenados de forma que $\mathbf{z}_{1}$ representa a maior quantidade de variação, $\mathbf{z}_{2}$ representa a segunda maior quantidade de variação, e assim por diante. Isto é.

$$
\operatorname{var}\left(\mathbf{z}_{1}\right) \geq \operatorname{var}\left(\mathbf{z}_{2}\right) \geq \cdots \geq \operatorname{var}\left(\mathbf{z}_{p}\right)
$$

em que var $\left(\mathbf{z}_{1}\right)$ denota a variancia de $\mathbf{z}_{i}$ no conjunto de dados em questăo. Os índices $\mathbf{z}_{i}$ são chamados de componentes principais.

Ao rcalizar uma análise de componentes principais, espera-se sempre que as variâncias da maioria dos índices sejam tão pequenas que possam ser desprezadas. Nesse caso, a variação do conjunto de dados podr sur lescrita adequadamente pclas poucas variáveis $\mathbf{z}$ cujas variâncias são consideradas não desprezíveís. Cin certo grau de economia é entāo atingido, já que a variação nas $p$ variáveis originais $\mathbf{x}$ fica representada por um menor número de variáveis $\mathbf{z}$.

\section{Procedimento para PCA}

Suponha $n$ observaçoes representadas por $p$ variáveis, como indicado na tabela 3.1. A primeira componente principal é portanto, a combinação linear das variáveis $\mathbf{x}_{1}, \mathbf{x}_{2}, \ldots, \mathbf{x}_{p}$, dada por:

$$
\mathbf{z}_{1}=a_{11} \mathbf{x}_{1}+a_{12} \mathbf{x}_{2}+\cdots+a_{1 p} \mathbf{x}_{p}
$$

que retém a máxima variação para as $n$ observações e é sujeita à condição:

$$
a_{11}^{2}+a_{12}^{2}+\cdots+a_{1 p}^{2}=1
$$

Portanto, a variáncia de $\mathbf{z}_{1}, \operatorname{var}\left(\mathbf{z}_{1}\right)$, é a maior possivel, considerando essa restrição para as constantes $a_{1 j}$. A restrição ć introduzida porque, caso contrário, var $\left(\mathbf{z}_{1}\right)$ pode aumentar simplesmente por consequência do aumento de qualquer um dos valores $a_{13}$. A segunda componente principal,

$$
\mathbf{z}_{2}=a_{21} \mathbf{x}_{1}+a_{22} \mathbf{x}_{2}+\cdots+a_{2 p} \mathbf{x}_{p},
$$


Tabcla 3.1: Dados para uma PCA.

\begin{tabular}{ccccc}
\hline Obscrvação & $\mathbf{x}_{1}$ & $\mathbf{x}_{2}$ & $\ldots$ & $\mathbf{x}_{p}$ \\
\hline 1 & $x_{11}$ & $x_{12}$ & $\ldots$ & $x_{1 p}$ \\
2 & $x_{21}$ & $x_{22}$ & $\ldots$ & $x_{2 p}$ \\
$\vdots$ & $\vdots$ & $\vdots$ & & $\vdots$ \\
$n$ & $x_{n 1}$ & $x_{n 2}$ & $\ldots$ & $x_{n p}$ \\
\hline
\end{tabular}

\section{?}

é aquela cuja variância, $\operatorname{var}\left(\mathbf{z}_{2}\right)$, é a maior possível sujeita à restrição:

$$
a_{21}^{2}+a_{22}^{2}+\cdots+a_{2 p}^{2}=1
$$

e também à condição de que $z_{1}$ e $z_{2}$ sejam não correlacionadas. A terceira componente principal,

$$
\mathbf{z}_{33}=a_{31} \mathbf{x}_{1}+a_{32} \mathbf{x}_{2}+\cdots+a_{3 p} \mathbf{x}_{p},
$$

é aquela cuja variancia $\operatorname{var}\left(\mathbf{z}_{3}\right)$. ć a maior possível sujeita à restriçào:

$$
a_{31}^{2}+a_{32}^{2}+\cdots+a_{3 p}^{2}=1
$$

e também à condição do quo $\mathbf{z}_{3}$ năo é correlacionada com $\mathbf{z}_{2}$ e $\mathbf{z}_{1}$. As outras componentcs principais sâo defindas de maneira análoga. Se existirem $p$ variáveis originais $\mathbf{x}$, poderão ser encontradas $p$ componentes principais.

\section{Autoestrutura da PCA}

De fato, a realizaçäo de una PCA resume-se em encontrar os autovalores da matriz de covariância dos dados, quo é simétrica e tem a forma:

$$
\mathbf{C}=\left[\begin{array}{cccc}
c_{11} & c_{12} & \ldots & c_{1 p} \\
c_{21} & c_{22} & \ldots & c_{2 p} \\
\vdots & \vdots & & \vdots \\
c_{p 1} & c_{p 2} & \ldots & c_{p p}
\end{array}\right]
$$

em que o elemento da diagonal principal $c_{i i}$ é a variância de $\mathbf{x}_{i}$ e $c_{i j}(i \neq j)$ é a covariância das variáveis $\mathbf{x}_{2}$ e $\mathbf{x}_{3}$.

É importante observar. porćm, que as variáveis $\mathbf{x}_{1}, \mathbf{x}_{2}, \ldots, \mathbf{x}_{p}$ devem ser padronizadas para que tenham média igual a 0 e variância igual a 1 antes de proceder com a análise, para 
evitar que uma determinada variável tenha uma infuência excessiva nas componentes principais. Dessa forma, após feita a codificaçăo dos dados, a matriz de covariância $\mathbf{C}$ tcrá a forma:

$$
\mathbf{C}=\left[\begin{array}{cccc}
1 & c_{12} & \ldots & c_{1 p} \\
c_{21} & 1 & \ldots & c_{2 p} \\
\vdots & \vdots & & \vdots \\
c_{p 1} & c_{p 2} & \ldots & 1
\end{array}\right]
$$

que coincide com a matriz de correlação dos dados, onde $c_{i j}=c_{j i}$ é a correlação entre $\mathbf{x}_{i}$ e $\mathbf{x}_{j}$. Em outras palavras. a PCA é realizada sobre a matriz de correlaçấo dos dados. Nesse caso, a soma dos elementos da diagonal principal é igual a $p$, ou seja, ao número de variáveis originais.

Nesse caso, o problema de autovalor, comumente referenciado em álgebra linear (Graybill 1983), resume-se cm resolver a seguinte equação:

$$
\mathbf{C A}=\mathbf{A} \Lambda
$$

em que $\mathbf{A}=\left[\mathbf{a}_{1}, \mathbf{a}_{2}, \ldots, \mathbf{a}_{i}, \ldots, \mathbf{a}_{i}\right]$ e $\mathbf{\Lambda}=\operatorname{diag}\left[\lambda_{1}, \lambda_{2}, \ldots, \lambda_{i}, \ldots, \lambda_{p}\right]$. O problema tem soluçós não triviais (isto ć. $\mathbf{a}_{i} \neq \mathbf{0}$ ) somente para valores especiais de $\lambda$, os quais são chamados de autovalores da matriz de correlação $\mathbf{C}$. Os valores de a associados aos autovalores são chamados de autovetores. A matriz de correlação é caracterizada por autovalores reais não negativos.

Sendo $\lambda_{1} \geq \lambda_{2} \geq \cdots \geq \lambda_{2} \geq \cdots \geq \lambda_{p}$ os autovalores da matriz de corrclação $\mathbf{C} \mathrm{c}$ $\mathbf{a}_{1}, \mathbf{a}_{2}, \ldots, \mathbf{a}_{p}$ os autovetores associados, respectivamente, pode-se observar alguns resultados importantes obtidos a partir da antoestrutura da PCA (Manly 1986):

- As variâncias das componentes principais são iguais aos autovalores da matriz de correlaşào $\mathbf{C}$, em que $\lambda_{i}$ ć o autovalor relativo à i-ésima componente principal.

$$
\mathbf{z}_{i}=a_{i 1} \mathbf{x}_{1}+a_{i 2} \mathbf{x}_{2}+\cdots+a_{i p} \mathbf{x}_{p}
$$

Em particular: $\operatorname{var}\left(\mathbf{z}_{i}\right)=\lambda_{i}$.

- As constantes $a_{i 1}, a_{i 2}, \ldots a_{i p}$ são os elementos do $i$-ésimo autovetor correspondente.

\section{Reconstruçāo do Vetor de Dados Original}

Para reconstruir o vetor de dados original $\mathbf{x}$, cxatamente a partir das componentes principais $\mathbf{z}_{i}$, procede-se cono segue. Primeiramente, combina-se as componentes principais 
$\left\{\mathbf{z}_{i} \mid i=1,2, \ldots, p\right\}$ em uma única matriz, como mostrado por:

$$
\mathbf{Z}=\left[\mathbf{z}_{1} \cdot \mathbf{z}_{2}, \ldots, \mathbf{z}_{p}\right]^{T}=\left[\mathbf{x}^{T} \mathbf{a}_{1}, \mathbf{x}^{T} \mathbf{a}_{2}, \ldots, \mathbf{x}^{T} \mathbf{a}_{p}\right]=\mathbf{A}^{T} \mathbf{x}
$$

A seguir, multiplicando ambos os lados da equação (3.10) pela matriz $\mathbf{A}$ e usando a propriedade que $\mathbf{A}$ é ortogonal, isto é, $\mathbf{A}^{T}=\mathbf{A}^{-1}$, o vetor $\mathbf{x}$ de dados originais pode ser reconstruído da seguinte forma:

$$
\mathbf{x}=\mathbf{A Z}=\sum_{i=1}^{p} \mathbf{z}_{i} \mathbf{a}_{i}
$$

\section{Redução de Dimensionalidade}

Do ponto de vista do reconhecimento de padrões estatístico, o valor prático da técnica PCA é fornecer uma técnica efetiva para redução de dimensionalidade. Em particular, pode-se reduzir o número de caractorísticas necessárias para representação efetiva dos dados, descartandose aquelas combinaçōes lineares com baixas variancias e retendo-se somente aqueles termos que tiverem variâncias significativas.

Sejam $\lambda_{1}, \lambda_{2} \ldots \lambda_{m}$ \& $m$ maiores autovalores da matriz de correlação C. Pode-se aproximar o vetor de dados $\mathbf{x}$ truncandò-se a expansāo da equaçāo (3.11) depois de $m$ termos, como segue:

$$
\mathbf{x}^{\prime}=\sum_{i=1}^{m} \mathbf{z}_{i} \mathbf{a}_{i}, \quad m<p .
$$

O erro que ocorre nesta aproximação é dado por:

$$
c=\mathbf{x}-\mathbf{x}^{\prime}
$$

en que $\mathrm{x}$ é o vetor de darlos originais $\mathrm{e} \mathrm{x}^{\prime}$ é o vetor de dados aproximados.

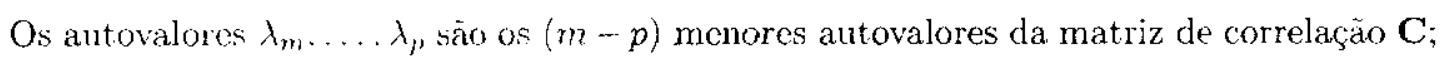
eles correspondem aos tornes lescartados da expansão da equação (3.12), usada para construir o vetor aproximado $x^{\prime}$. Quanto mais próximos esses autovalores estiverem de zero, mais efetiva scrá a redução de dimensionalidade (resultante da aplicaşão da PCA ao vetor de dados $\mathbf{x}$ ) na preservação da informação contida nos dados de entrada.

A utilização dá técnica PCA pode, então, ser resumida através dos seguintes passos:

1. Padronize os dados correspondentes às variáveis $\mathbf{x}_{1}, \mathbf{x}_{2}, \ldots, \mathbf{x}_{p}$, para que estes tenham média igual a 0 e variância igual a 1 .

2. Calcule a matriz de correlação $\mathbf{C}$. 
3. Encontre os autovalor'es $\lambda_{1}, \lambda_{2}, \ldots, \lambda_{p}$ da matriz $\mathrm{C}$ e seus correspondentes autovetores $\mathbf{a}_{1}, \mathbf{a}_{2}, \ldots \mathbf{a}_{p}$. Os coeficientes da $i$-ésima componente principal são dados por $\mathbf{a}_{i}$, encuanto $\lambda_{i}$ é a sua variância.

4. Descarte as componentes que acumulem uma pequena proporção da variação dos dados. Por exemplo, se os dados originais tiverem 20 variáveis e as três primeiras componentes principais forem responsáveis por $90 \%$ do total da variância, as outras 17 componentes principais podem ser ignoradas.

Maiores detalhes sobre a PCA podem ser encontrados em (Kendall 1980), (Johnson \& Wichern 1998), (Manly 1986) e (Oliveira 1997).

\subsubsection{Técnicas de Agrupamento (Clustering)}

Suponha uma amostra de $n$ objetos, cada qual contendo valores para $p$ variáveis. Técnicas de agrupamento têm como objetivo básico descobrir grupos naturais desses objetos ou variáveis tal quuc objetos on variárcis "similares" estejam no mesmo grupo, sendo, para isso, necessário definir, primeiramente, uma escala quantitativa para medir a associaçào (similaridade) entrc os objetos.

Técnicas de agrupamento podem ser consideradas técnicas de classificação não supervisionada (Diday \& Simon 1980). No cutanto, vale a pena ressaltar que métodos de classificaçāo supervisionada e análise de agrupantento são técnicas completamente distintas. O objetivo da classificação supervisionada d́, dadas $K$ classes, scndo $K$ conhecido, associar cada nova observaçăo a uma dessas classes. Além disso, uma técnica de classificação supervisionada requer requer conhecimento a priori sobre as classes, geralmente na forma de uma amostra para cada classe. Já em técnicas de agrupanento, não é feita nenhuma suposiçào envolvendo o número ou a estrutura das classcs. Os agrinjannentos são feitos com base en similaridades ou em distâncias. As entradas requeridas para essa análise são, portanto, medidas de similaridades ou dados a partir dos quais possam ser computadas tais medidas.

Mesmo sendo difícil definir precisamente o significado de "cluster", foram propostos vários métodos que realizam tal análise. Em (Cormack 1971) são descritos alguns dos métodos mais utilizados.

\section{Medidas de Similaridade}

Define-se uma medida de "proximidade" on "similaridade" com o objetivo de produzir uma estrutura de grupos a partir de um conjunto de dados. A escolha de uma medida de simi- 
laridade envolve consideraçoos inportantes como: a natureza das variáveis (discreta, contínua, binária) e a escala das mediçós.

Quando o objetivo da análise de agrupamento é agrupar objetos, a proximidadc é quase sempre indicada por algum tipo de distância. Já quando a meta for agrupar variáveis, geralmente os coeficientes de correlação são levados em conta. A distância mais utilizada para a análise de cluster é a distância euclidiana entre duas observações (objetos) de mesma dimensão.

Antes do cálculo das distâncias, ć usual padronizar as variáveis para que todas clas tenham importância equivalente na determinação dessas medidas. Isso pode ser feito padronizando-se as variáveis para que essas tenham média 0 e variância 1 . Alternativamente, cada variável pode ser codificada para ter o mínimo de () e o máximo de 1.

Quando os objetos nào pudercm ser representados por medidas $p$-dimensionais significativas, as distâncias entre parrs de objet os podem ser calculadas, por excmplo, com base na presença ou ausência de certas características. Obviamente, objetos similares terão mais características em comum do que os objetos não similares. A presença ou ausência de uma característica pode ser descrita matematicamonto através da introdução de uma variável binária que assume valor 1 , se a característica estiver prescnte, $c$ valor 0 , se a característica estiver ausente.

\section{O Método $k$-médias}

$\mathrm{Na}$ sua forma geral. o $k$-médias é um algoritmo iterativo utilizado para particionar $n$ pontos de dados $\mathrm{em} K$ subconjuntos (grupos) disjuntos $S_{j}$ contendo, cada $u \mathrm{~m}, n_{j}$ pontos de dados, de modo a minimizar a soma dos quadrados das distâncias dentro dos grupos, dada por:

$$
D=\sum_{j=1}^{K} \sum_{i \in S_{j}}\left|\mathbf{x}_{i}-\mathbf{m}_{j}\right|^{2},
$$

onde $\mathbf{x}_{i}, i=1.2, \ldots, n$, é un vetor que representa o $i$-ésimo ponto de dados do conjunto $\mathbf{e}_{j}$ é o centróide dos pontos cle dados em $S_{j}$, geralmente calculado a cada iteração como o vetor de médias destes pontos. O algorit n110 $k$-médias é apresentado a seguir.

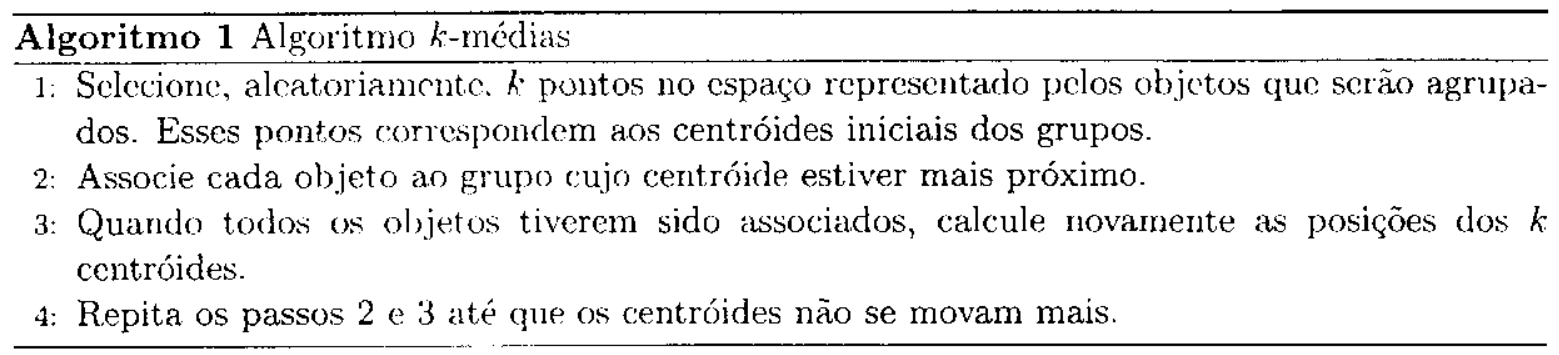


De fato, nāo foi realizado, neste trabalho, um estudo mais aprofundado dos fundamentos do algoritmo k-médias. Porćm, alguns resultados obtidos por essa técnica também são apresentados no Capítulo 8 . por se tratar de um algoritmo básico bastante utilizado em análises comparativas envolvendo métodos auto-organizáveis.

\subsection{Redes Neurais Artificiais}

Redes neurais artificiais (RNAs), com seu denso paralelismo, têm provado ser uma abordagem promissora en várias aplicaçōes na árca de computação visual, tendo obtido sucesso na solução de problemas para us quais as técnicas tradicionais têm falhado ou mostrado ser ineficientes. A investigação das RNAs nesse contexto tem recebido grande atenção, uma vez que esses modelos apresentan ontras características adequadas ao processamento de imagens, além do paralelismo. Por exemplo. RNAs permitem a representação distribuída das informações, facilidade de aprendizagem por excrnplos, alta capacidade de generalização e uma certa tolerância a falhas. Dentre as aplicaçoes das RNAs envolvendo análise de imagens, destacarn-se operações de robótica, inspeçào visual. controle de veículos autônomos e muitas outras.

Modelos de redes neurais săo compostos, basicamente, por processadores elementares, chamados de nós ou newronios, altamente conectados (Hertz, Krogh \& Palmer 1991), (Kosko 1991). (Kohonen 1989). (Pao 1989). A arquitetura e a dinâmica de algumas redes neurais tentam imitar o processamento das informações nos neurônios biológicos. Na maioria dos casos, a arquitetura altamente conexionista faz com que a rede neural seja robusta, enquanto que o processamento paralelo possibilita o sistema a produzir saídas em tempo real.

\subsubsection{Processo de Aprendizado para Redes Neurais}

A propriedade nais importante de uma rede neural é a habilidade para aprender e melhorar o seu desenipeniso por meio do aprendizado. Uma rede neural aprende via um processo iterativo de ajustes aplicados aos pesos sinápticos de suas conexōes, tornando-se mais conhecedora de seu ambiente a cada iteração.

Existem muitas nocies associadas ao conceito de aprendizado, o que torna difícil a sua definição de uma mancira precisa (Minsky 1961). Uma definiçāo de aprendizado para redes neurais proposta por (Nondel \& McLaren 1970) é apresentada a seguir:

"Aprendizado é um processo pelo qual os parâmetros livres (pesos sinápticos) de uma rede neural são adaptados por mcio de um processo contínuo de estímulos dados pelo ambientc no qual a rede está incorporada. O tipo de aprendizado é determinado pelo modo que as mudanças 
nos parâmctros são realizadas." Essa definição implica nas seguintes etapas de eventos:

1. A rede neural é estimulada por um ambiente (apresentação de um exemplo de treinamento).

2. A rede neural muda como resultado desse estímulo (adaptação dos pesos sinápticos).

3. A rede neural responde do um novo modo ao ambiente, em função das mudanças que ocorreram na sua estrutura interna.

Considerando-se um par de neurônios $x_{k}$ e $x_{j}$ conectados por uma ligação com peso sináptico $w_{k j}$, e denotando-se $w_{k j}(n)$ como o valor do peso sináptico $w_{k j}$ no tempo $n$ e $\Delta w_{k j}(n)$ como o ajuste a ser aplicado a esse peso, tem-se:

$$
w_{k j}(n+1)=w_{k j}(n)+\Delta w_{k j}(n)
$$

em que $w_{k j}(n)$ e $w_{k j}(n+1)$ podem ser vistos como os valores antigo e novo do peso sináptico $w_{k j}$. Em particular, o ajuste $\Delta w_{k j}(n)$ é computado como resultado de um estimulo do ambiente (evento 1), e o valor atualizado $w_{k j}(n+1)$ define a mudança feita na rede devido a esse estímulo (evento 2). O evento 3 acontece quando a nova rede, operando com os pesos sinápticos atualizados, é reavaliada.

Um conjunto prescrito de regras bem definidas para a solução de um problema de aprendizado é chamado de algoritmo de aprendizado. Existem vários algoritmos de aprendizado que podem ser utilizados em projetos de redes neurais, diferindo uns dos outros, basicamente, pela maneira que são formulados os ajustes aplicados aos pesos sinápticos da rede. Um outro fator a scr considerado é o paradigma de aprendizado, ou seja, o modelo do ambiente no qual a rede neural está operando. Nesse contexto. o aprendizado pode ser supervisionado, nāo supervisionado ou com reforço, os quais serão descritos a seguir.

\section{Paradigmas de Aprendizado em Redes Neurais}

\section{$\diamond$ Aprendizado Supervisionado}

A disponibilidade de um professor externo caracteriza o aprendizado supervisionado. Em termos conceituais, podir-se dizer que o professor é aquele que tem conhecimento do ambiente, o que é representado por um conjunto de exemplos entrada-saída.

Durante o processo de aprendizado, o professor é capaz de fornecer à rede neural a resposta desejada para un detcrminado conjunto de trcinamento. Tal resposta representa a ação ótima a ser executada pela rede. 
Os parâmetros dat rede são ajustados levando-se em conta, geralmente, o vetor de treinamento e o crro, sendo que o erro é dado pela diferença entre a resposta atual c a resposta desejada. Esses ajustes são realizados, quase sempre, iterativamente, passo a passo, com o objetivo de fazer com que a rede emulc o professor. Em outras palavras, o conhecimento disponível ao professor é transferido para a rede neural da forma mais completa possível. Quando essa condiçāo for atingida, podc-sc dispensar o professor e deixar que a rede, completamente por si só: lide com o ambiente.

Um exemplo represcritativo de rede neural que utiliza o paradigma de aprendizado supervisionado é a rede Perceptron Multi-Camadas (MLP) (Rumellart, Hilton \& Williams 1986).

\section{$\diamond$ Aprendizado Não Supervisionado}

No aprondizado não sujervisionado (ou auto-organizável) não existe professor externo. Em outras palavras, não cxistem exemplos específicos da função a ser aprendida pela rede. A rede deve aprender com basc cm mna medida de qualidade, ou seja, os pesos sinápticos da rede devem ser ajustados de mancira a otimizar tal medida.

O paradigma de aprendizado não supervisionado apresenta, como principais sub-grupos, o aprendizado hebbiano (o aprendizado competitivo, que scrāo discutidos no decorrer deste capítulo.

\section{$\diamond$ Aprendizado com Reforço}

No aprendizado con reforço a aprendizagem ć realizada por meio de mapcamentos entrada-saida que caracterizam um processo de tentativa c erro projetado para maximizar um detcrminado critério de desmpenho, chamado de sinal de reforço. Se uma açäo executada pela rede neural é seggudia por um estado satisfatório, entäo, a tendência da rede para produzir açucla ação ć fortalccida on roforçada. Caso contrário, a mesma tendôncia é enfraquecida (Haykin 1994).

A seguir scrāo aprescntadas duas abordagens de aprendizado não supervisionado que são utilizadas nos nodelos de redes neurais descritos neste trabalho.

\section{Exemplos de Tipos de Aprendizado Não Supervisionado}

Uma vez que o enfoque cleste trabalho consiste na utilização de modelos de redes neurais auto-organizáveis, a seguir serão descritos dois tipos de aprendizado que alguns destes modelos implementam.

\footnotetext{
${ }^{1}$ Do original, em inglés. Multi Layer Perceptron.
} 


\section{Aprendizado Hebbiano}

O postulado de Hebb (Hebb 1949) é o mais antigo entre as regras de aprendizado para redes neurais e consiste em:

"Quando um axônio da célula $A$ está suficientemente próximo para excitar uma célula $B$ e repetidamente tenta cxcitá-la, algum processo crescente ou mudanças metabólicas ocorrem em ambas as células."

Transformando a declaração acima em duas regras (Changeux \&. Danchin 1976), segue que:

1. Sc dois neurônios ligarlos por uma sinápse sāo ativados simultaneamente (sincronamente), a intensidado dessa sinápse (conexão) é aumentada.

2. Se dois neurônios ligados por uma sinápse são ativados assincronamente, a intensidade dessa sinápse é diminuída ou climinada.

De acordo com H(b), o ajustc aplicado ao peso sináptico $w_{k j}$ no tempo $n$ é expresso na forma:

$$
\Delta w_{k j}(n)=\eta y_{k}(n) x_{j}(n)
$$

onde $\eta$ ć uma constante pusitiva que determina a velocidade do aprendizado, $y_{k}(n)$ é a saída do neurônio $k$ no tempo $n$ e $x,(n)$ ó u j-ésimo elemento do vetor de entrada no tempo $n$.

Dentre as principais aplicaçōes do aprendizado hebbiano, destacam-se a extraçāo de características de dados e técnicas de memória associativa. Exemplos de redes neurais que implementan esse: tipo cle aprentizado são os modelos de Hopfield (Hopfield 1982) e redes PCA (ver seção 3.3.3).

\section{$\diamond$ Aprendizado Competitivo}

No aprendizado competitivo. os neurônios de saida de uma rede neural competcm entre si para ser o neurônio que scrá at ivado. Ao contrário de uma rede neural baseada no aprendizado de Hebb, onde vários neurònios podem ser ativados simultaneamentc, no aprendizado competitivo, apenas um neurônio é ativado por vez. Tal característica torna esse tipo de aprendizado bastante adequado para realizal extraya de características em um conjunto de dados com a finalidade de classificar os padrôes de entrada (Haykin 1994).

Existem dois elementos básicos em uma regra de aprendizado competitivo: 
- Um conjunto de neurônios que são idênticos, diferindo uns dos outros somente pelos seus pesos sinápticos distribuídos aleatoriamente. Devido a essa diferença, os neurônios da rede. respondem de forma diferente a cada padrão de entrada.

- Um mecanismo que permite aos neurônios competirem para responder corretamente a um dado subconjunto de entrada, tal que somente um neurônio de saída seja ativado por vez.

O neurônio que ganha a competição é chamado de neurônio vencedor.

O neuronio $j$. vencedor, é aquele cujo o nivel de atividade $v_{j}$, para uma determinada entrada, é o maior entre todos os neurônios da rede. Neste caso, o neurônio que ganha a competição ganha também o direito de ter os seus pesos sinápticos ajustados. Em alguns modelos competitivos, além do neurônio vencedor, alguns neurônios mais próximos em sua vizinhança também têm os seus pesos atualizados. Este é caso, por exemplo, dos Mapas Auto-organizáveis $(\mathrm{SOM})^{2}$ propostos por Kohonen (Kohonen 2001).

Outros exemplos de redes neurais que implementam o aprendizado competitivo sāo os modelos da família ART. Aspectos importantes destes serão apresentados a seguir neste capítulo, uma vez que una rede neural desta família foi utilizada, como uma abordagem alternativa, nos experimentos de segmentação de imagens, como será apresentado no Capítulo 8.

\subsubsection{Modelos da Família ART}

A Teoria da Ressonancia Adaptativa (ART) foi desenvolvida por Carpenter e Grossberg (Carpenter \& Grossberg 1987b). As redes neurais projetadas sob esse paradigma, denominadas de redes da Família ART, foram desenvolvidas de modo a permitir que o usuário controle o grau de similaridade entre os padrões alocados em um mesmo grupo (cluster).

À medida que uma rede ncural ć treinada para identificar agrupamentos, um mesino padrão pode ser apresentado várias vezcs. Na primeira apresentação, cada padrão é associado a uma unidade de cluster. inicialmente, podendo ser deslocado para outra unidade nas apresentaçōes seguintes. Isso pode acontecer devido às mudanças aplicadas nos pesos dos clusters iniciais, que tiveram que se adaptar a outros padrões no decorrer do treinamento. Todavia, se um padrão oscilar. a cada inpresentação, entre unidades de cluster diferentes, a rede pode ser considerada instável.

Algumas redes atingem estabilidade por meio da redução gradual das suas taxas de aprendizado à medida que o mesmo conjunto de padrões é apresentado várias vezes. Entretanto, esse mecanismo dificulta o aprendizado de um novo padrão que seja apresentado depois que um certo

\footnotetext{
${ }^{2}$ Do original, cm ingless. Self-Organizing Maps.
} 
número de épocas de treinamento tenha ocorrido. A habilidade de uma rede neural para responder a (apronder) um novo padrào de forma satisfatória, em qualquer estágio de treinamento, é denominada de plasticidade. Redes neurais ART foram projetadas para serem, ao mesmo tempo, estáveis c plásticas, de modo a solucionar o dilema Estabilidade - Plasticidade, que será descrito a seguir.

\section{Dilema Estabilidade - Plasticidade}

Dentre as várias habilidades inerentes à mente humana, uma das mais interessantes $\mathrm{e}$ fundamentais para o indivíduo es a capacidade de aprender e de continuar aprendendo coisas novas. sem esquecer o que já foi aprendido no passado. Um exemplo, bastante comum, é a capacidade de um individuo reconhecer seus pais, mesmo sem tê-los visto por algum tempo e, durante este periodo, tor conbcido uma variedade de rostos (ou pessoas) diferentes. Sistemas complexos com arquitetura paralela, tais como o cércbro, devem ter algum meio de manter a estabilidade cm todos os níveis para desempenhar bem suas tarefas. Seria, portanto, de grande interesse que um sistema computacional tivesse esta característica (Freeman \& Skapura 1991).

Quando sc projeta un sistena computacional neural para que este desempenhe tarefas de classificação de padrões, primeiramente cria-se um conjunto de exemplos (ou padröes) para ser utilizado no treinanento do sistema. Durante o treinamento, as informações apresentadas ao sistenla em questão serăo incorporadas a este por meio do processo de ajuste dos pesos sinápticos associados aos neurônios da rede. Uma vez terminado o processo de treinamento, o sistcma está pronto para scr colocado en prática e, a partir de então, nenhum ajuste adicional é permitido no conjunto de pesos. Estc tipo de situaçāo ć aceitável em um ambiente (ou domínio de aplicação) estável, ou seja, qux possui limites bem definidos. Em ambientes estávcis é possivel, geralmente, definir um conjunto de treinamento adequado para qualquer que scja o problema a ser resolvido.

Infelizniente. o que acontece na maioria das situaçōes do mundo real ć que os ambientes sāo dinâmicos (não-estáveis) e, na maioria dos casos, os limites dos ambientes não são conhecidos. Como exemplo de ambiente dinâmico, pode-se considerar a situação em que se tenha que reconhecer silhuetas (formas) de algumas classes de aeronaves. As imagens apropriadas podem ser usadas para treinar o sistema neural, e esta tarefa, dependendo da arquitctura deste sistema, consumirá algum tempo. Após o processo de treinamento, nenhuma outra modificação nos pesos é permitida. Sc $\mathrm{cm}$ algum momento no futuro, um outro modelo de aeronave de uma mesma classe já conhecida tornar-se operacional, e se os usuários do sistema desejarem adicioná-lo ao conjunto de exemplos de treinamento que já possuem, é necessário que se faça um novo treinamento do sistema utilizando os exemplos que já foram utilizados anteriormente, 
mais os exemplos do novo modelo. O novo treinamento deve ser feito desta forma, pois caso seja feito somente com o novo modelo de aeronave, o sistema neural assimilará muito bem este, mas, em contrapartida, os outros padroes que foram usados no primeiro trcinamento ficarão afetados, talvez, em sua totalidade.

Esse tipo de situação acontece porque o sistema computacional neural, não possuindo nenhum conluecimento a respeito da nova entrada, e não dispondo de um mecanismo de generalizaçào capaz de reconhecer o novo padrão de entrada, tenta ajustar-se à nova situação "esquecendo" o conhccimento previamentc adquirido. Essas características podem ser observadas em alguns sistemas computacionais. tais como em redes neurais Perceptron Multi-Camadas(MLP) (Rumclhart, Hilton \& Willians 1986) e Mapas Auto-Organizáveis (SOM) (Kohonen 1988).

Carpenter e Grosisberg (Carpenter \& Grossberg 1987b), preocupados com a estabilidade em sistcmas auto-organizávcis. deschvolveram a Teoria da Ressonância Adaptativa (ART), a partir do que eles chamaram de dilema Estabilidade-Plasticidade. Esse dilema pode ser compreendido a partir do uma sćric de questōes:

- Como um sistema (capaz de aprender) pode continuar sendo adaptativo (plástico) em resposta a ma entrada significativa (nova) e, ao mesmo tempo, manter-se estável em resposta a uma entradia irrelevante (conhecida)?

- Como o sistcma será capaz dle alternar entre os modos plástico e estável ?

- Como o sistenta poderá reter as informações aprendidas anteriormente e continuar aprendendo novas informaçòes?

Dessa forma, quando um sistema "inteligente" aprende novas informaçôes, sem perder aquelas previamente aprendidas. diz-se que este sistema é dotado de plasticidade, ou é plástico. Por outro lado, quandio nàr há prerdas significativas de informaçōes, ou seja, nào há diferentes interpretaçòes para um mesrno padrāo durante diferentes estágios de treinamento, diz-se que o sistema em questão é dotado de estabilidade, on é estável. A idéia central, para resolver o dilema estabilidade-plasticidade é adicionar entre a camada de saída (competitiva) do sistema computacional neural e a camada de entrada um mecanismo de retorno (feedback) para facilitar a adaptação a uma situação nova. sem perder o conhecimento anterior, permitindo que seja possivel alternar entre os modos estável e plástico, durante a fase de treinamento.

\section{Exemplos de Modelos ART}

Desde o surgimento da teoria da ressonância adaptativa, vários modelos fundanentados nesta teoria foram desenvolvidos. O primeiro modelo da família ART foi o ART1 (Carpenter \& 
Grossberg 1987b), que reconhece agrupamentos (clusters) de padrōes em seqüências arbitrárias de valores binários. A rede ART2 (Carpenter \& Grossberg 1987a), foi desenvolvida com o objetivo de abranger um número maior de situações do mundo real, para isso aceitando sequiências arbitrárias de valores reais.

Tanto o modelo ART1 quanto o ART2 foram projetados para funcionar com algoritmo de aprendizado não supcrvisionado. Um outro modelo, também de paradigma não supervisionado, é o ART3 (Carpenter \& Grossberg 1990). Este, diferente dos dois citados anteriormente, é fundamentado no processo de sinápses complexas, que inclui um modelo de neurotransmissores químicos, e fornece um mecanismo de busca paralela para reconhecimento distribuído em uma rede neural hierárquica multi-nivel.

A primeira rede da família ART a implementar o aprendizado supcrvisionado e incremental foi a rede ARTMAP (Carpenter, Grossberg. Neurophysiol \& Reynolds 1991). Esta, por sua vcz, foi projetada para reconhecer agrupamentos em resposta a vetores de entrada binários. O modelo Fuzzy ART (Carpenter: Grossberg \& Rosen 1991b) combina a abordagem ART com fundamentos dia lógica nebulosia ( fuzzy) (Zadeh 1994) e será abordado com mais detalhes no decorrer deste capítulo, por t.cr sido utilizado nos expcrimentos com imagens do presente trabalho. Há também o modelo Finzy ARTMAP (Carpentcr, Grossberg, Markuzon, Reynolds \& Rosen 1991), que é umá versão análoga ao ARTMAP para padrões de entrada com valores reais.

Existem, ainda. muitas outras variaçōes importantes, tais como os modelos ART2-A (Carpenter, Grossberg \& Rosen 1991a). ARTSTAR (Hussain 1993), SMART (Bartfai 1994). HART (Bartfai 1995). ART-C. (He. Tan \& Tan 2002), Gaussian ART (GART) (Williamson 1996), Simplified ART (Baralli \& Alpaydin 1998), Self-Organizing SART (FOSART) e Fuzzy ART simétrica (S-Fuzzy ART). Esses quatro últimos modelos foram analisados com detalhes por Baraldi e colaboradores (Baraldi \&. Blonda 1999), (Baraldi \& Alpaydin 2002a), (Baraldi \& Alpaydin 2002b).

\section{Arquitetura básica dos modelos ART}

A arquitet ura das redes da farnília AR'T pode ser representada por um esquema geral (ver Figura 3.1), que envolve tress grupos de neurònios:

- camada $F_{1}$ : constituída das unidades de entrada e de interface;

- camada $F_{2}$ : constituída das unidades de cluster representadas pelas unidades $y_{j}$;

- unidade de Reset: implementa o controle do usuário sobre o grau de similaridade entre os padröes classificados em un mesmo cluster. 
Todas as unidades da camada $F_{1}$ estào unidas, por meio de conexões top-doun e bottomup, à camada $F_{2}$. Deve-se notar, na arquitetura apresentada, que a camada $F_{1}$ recebe como entrada um vetor $\mathrm{s} e$, produz como saída, um vetor $\mathbf{x}$, o que significa que poderá ocorrer algum pré-processamento nos dados de entrada. Para facilitar o entendimento, pode-se fazer uma divisão da camada $F_{1}$ em duas ${ }^{3}$ (Fausset 1994): $F_{1(a)}$, que incorpora o vetor de entrada original $s_{i}$, e $F_{1(b)}$, sub-camada intcrface, constituída pelas unidades $x_{i}$ pré-processadas (ver Figura 3.2).

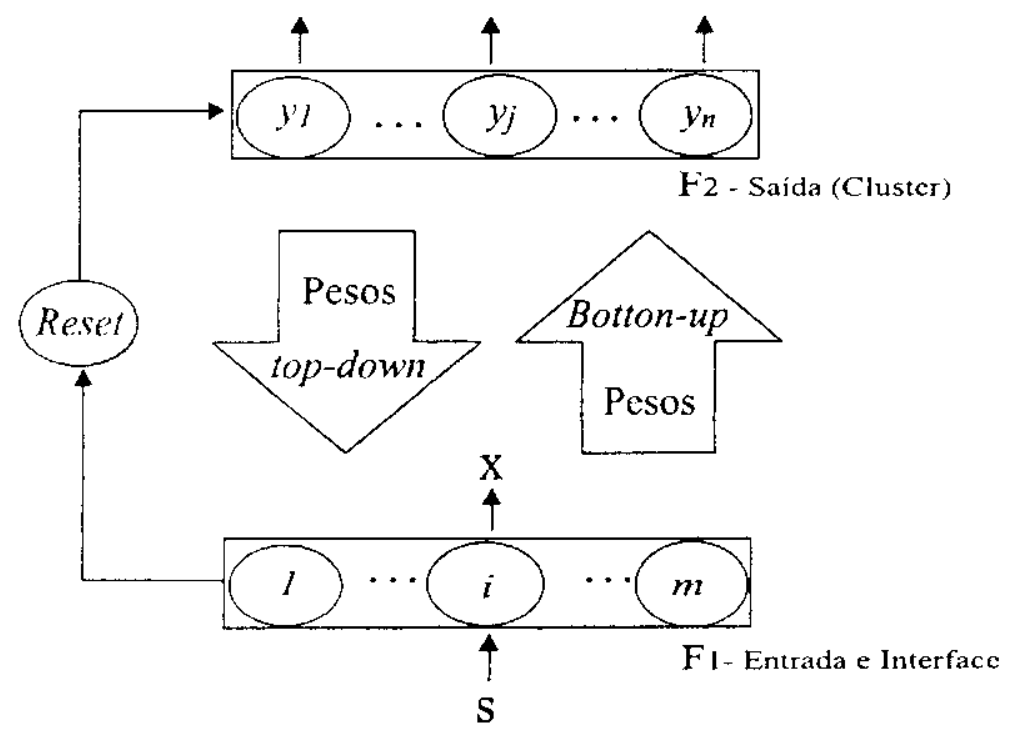

Figura 3.1: Estrutura básica de uma rede ART.

\section{Aprendizado nos modelos ART}

Os modelos ART podem implementar tanto o paradigma de aprendizado supcrvisionado quanto o não supervisionando. Além disso, são sistemas de aprendizado competitivo, isto é, os neurônios da camada de saída vão competir entre si pelo direito de aprender o dado de entrada submetido à redc. Outra característica é que o tipo de aprendizado implementado por esses modelos é incremental. o que significa que o sistema pode entrar no modo de treinamento a qualquer momento, possibilitando que a rede incorpore conhecimento novo, mantendo sua plasticidade e estabilidade (ver a Seção 3.3.2).

Nas redes ART, o período de ressonância corresponde a um processo adaptativo do sistema, que ocorre de duas formas. A primeira destas acontece se a rede aprendeu o suficiente para reconhecer os padroes submetidos à entrada. Neste caso, o processo de ressonância é realizado rapidamente reforçando seus pesos (ou memória da rede). A segunda maneira de o sistemá

\footnotetext{
${ }^{3}$ Isto foi feito por conveniencia a partir do modelo original (Carpenter \& Grossberg 1987b).
} 
entrar no estado de ressonânciá ocorre quando o padrão de entrada não é imediatamente reconhecido. Neste outro casos: a rede faz uma busca dentre seus padrões armazenados, na tentativa de encontrar um equivalente: caso não seja encontrada nenhuma equivalência, o sistema entra em estado de ressonância para aprender o novo padrão.

Normalmente, há dois modos de treinamento para as redes ART, considerando um mesmo modelo: modo de trcinamento rápido (fast learning) e modo de treinamento lento (slou learning). No modo de treinamento rápicko, os vetores de pesos são direcionados para seus valores ótimos em poucos ciclos de treinamento, por exemplo em um ou dois ciclos. No treinamento lento, os pesos são ajustados lentamente, em vários ciclos de treinamento, por exemplo em dez ciclos.

As redes da família ART foran projetadas para permitir que o usuário tenha a possibilidade de controlar o grau de similaridade entre os padrões agrupados em um mesmo cluster. Esse parâmetro de similaridade. nas redess ART, é o threshold( $\rho)$, ou parâmetro de vigilância, que, por sua vez, controla a resoluçăo do processo de agrupamento (i.e., o grau de similaridade entre os padrões colocados $\mathrm{cm}$ um mesmo cluster). O parâmetro de vigilância é o mais crítico dentre os parârretros dos modclos ART. assumindo valores entre 0 (zero) e 1 (um). Se $\rho$ assume um valor baixo (por exemplo, $\rho<0.3$ ). a resolução do processo de classificação será baixa, criando poucos clusters. Se, para a mesma aplicação, $\rho$ assumir urı valor alto (por exemplo, $\rho>0.9$ ), uma pequerra variação nos padrões de entrada pode levar à criação de novos clusters.

A condição de parada do trinamento nos modelos ART pode ser estabelecida de duas formas: a primeira. quandu não ocorrer mudança nos pesos após um determinado número de ciclos; a segunda quando um númcro máximo de ciclos for atingido. Caso todos os padrões apresentados à rede nào forem agrupados quando tcrminar os ciclos de treinamento, é preciso aumentar o múmero de clusters cm $F_{2}$, ou reduzir o valor de $\rho$, e reiniciar o algoritmo.

Na próxima scçào. será apresentado o modelo ART1, que é de fundamental importância para o entendimento do modelo Fuzzy ART, utilizado no presente trabalho. Para facilitar a compreensão dos algoritmos de aprendizagem dos modelos, o processo de treinamento foi dividido nos seguintes estágios:

- Inicializaçāo: os parâmetros e us pesos da rede são inicializados;

- Entrada: os padrões de treinamento são apresentados à entrada da rede;

- Busca: o neurônio com maior ativação é escolhido;

- Comparação (ou Reset): o neurônio, escolhido no estágio anterior, tem scus pesos avaliados com relaşão à similaridade com o padrão de entrada; 
- Adaptação: a regra de aprendizado é aplicada (processo de ressonância).

\section{ART1}

O modelo ART1 foi projetado para agrupar vetores de entradas binários, utilizando para isto o paradigma de aprendizado naao supervisionado e incremental. Além disso, o processo de treinamento nào exige que os padrōes de entrada possuam urna ordem fixa, ou que o número de padrōes seja pré-definido para treinamento. Dessa forma, mais padrōcs podem ser adicionados durante o processo de treinamento.

\section{$\diamond$ Arquitetura da rede ART1}

A arquitetura da rede ART1 consiste de unidades computacionais principais, denotadas por $F_{1}, F_{2}$ e Reset. e de midade suplementares, denotadas por $G_{1}$ e $G_{2}$ (ver Figura 3.2). Assim como descrito anteriomente. na Seção 3.3.2, a camada de entrada $F_{1}$ pode ser dividida em duas sub-camadas: $F_{1(a)}$ e $F_{1(b)}$. A sub-camada de interface $F_{1(b)}$, constituída pelas unidades $x_{i}$, combina sinais provenientes da camada $F_{1(a)}$, constituida pelas unidades $s_{i}$, e da camada $F_{2}$, constituida pelas unidades $y_{j}$. e isto é feito para tornar possível a comparação do sinal de entrada com a entrada pondcrada (reforçada) pelo vetor de peso da unidade que foi selecionada para adaptaçāo (aprendizado). Para comparar o grau de similaridade entre padrões, existem dois conjuntos de conexôes (cada $\mathrm{m}$ com seus próprios pesos), conectando as unidades de interface $x_{i}$ com as unidades de clusters $y_{3}$. As conexões que partem de $F_{1(b)}$ e vāo em direção à $F_{2}$ são reforçadas pelos pesos bottoml-up e assumem valores reais $b_{i j}$. As conexōes que partem da camada $F_{2}$ em direção à camada $F_{1(b)}$ são reforçados pelos pesos top-doun, que assumem valores binários $t_{j i}$. A canada $F_{2}$ é competitiva, ou seja, a unidade de cluster que possuir maior valor de ativaçào será escolhida para aprender o padrão de entrada.

Para cadd camada dos modelos ART1, existem unidades externas de controle, que são chamadas de unidades suplementares, representadas por $G_{1}$ e $G_{2}$. A função destas unidades é controlar o fluxo de dados através das camadas em cada fase de um ciclo de treinamento. Estas unidades também săo denominadas de unidades de controle de ganho (Gain Control Units) e serâo descritas mais adiant.c. juntamente com a finalidade dos sinais "+" e "-". Entre a camada de entrada e a camada de saída há também a unidade de Reset, que é responsável por calcular uma medida de similaridade relativa entre o vetor de cntrada e o protótipo armazenado no vetor de pesos associado ao neurônio vencedor, com o objetivo de comparar essa medida com o parâmetro de vigilância $(\rho)$.

Uma dificuldarle inerente à arquitetura do modelo ART1, é que as unidades que a compõem 


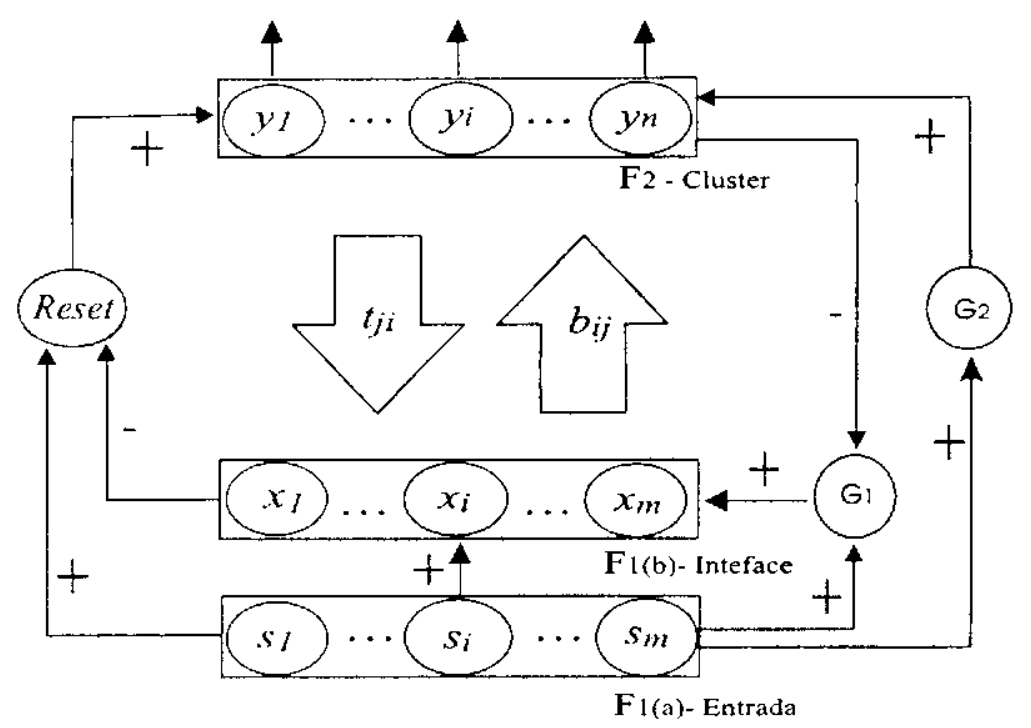

Figura 3.2: Arquitetura da rede ART1.

têm que responder de forma diferente aos diversos cstágios do processo ncural. Por exemplo, as unidades de $F_{1}$ devern estar ativas sempre que o sinal ć percebido na entrada $F_{1(a)}$ e nenhuma unidade de $F_{2}$ está ativa. As unidades de $F_{1(b)}$ devem estar ativas, se e somente se, recebem sinais de $F_{2}$ e da camada de entrada. A operação da unidade de Reset também possui um papel importante dentro do sistema de processamento neural, uma vez que essa unidade também deve inibir a unidade $F_{2}$, sob certas condições, e reativá-la em uma etapa mais adiante. Estes dois problemas podem ser compreendidos e resolvidos quando são adicionadas as unidades suplementares $G_{1}$ e $G_{2}$.

Dessa forma, as unidaries suplementares são importantes sob o ponto de vista teórico, uma vez que estas fornecem um mecanismo para o qual o algoritmo apresentado na Seção (3.3.2) possa ser implementado usando princípios de redes neurais.

Na Figura (3.2) os sinais excitatórios são representados por "+", c os sinais inibitórios são representados por "." - Un sinal. seja este qual for, é enviado sempre que uma unidade está ativa. Cada unidade das camadas $F_{\mathrm{l}(b)}$ c $F_{2}$ recebem três sinais que vêm de outras unidades. Por exemplo, $F_{7(b)}$ pode receber sinais de $F_{1(a)}, F_{2}$ (através dos pesos top-down) e $G_{2}$. Da mesma forma, $F_{2}$ pode rccebcr sinais de $F_{1(b)}$, Reset e $G_{1}$ (ver Figura 3.2). Cada uma das camadas $F_{1(b)}$ (através dos pesos bottom-up) e $F_{2}$ devem receber pelo menos dois sinais excitatórios para tornarem-se ativas, considerando três possívcis fontes de sinais. Esta situação é conhecida por "regra dos $2 / 3$ (dois terços)". 


\begin{tabular}{|c|c|}
\hline Variáneis & Descriçâa \\
\hline$m$ & dimensão do vetor de entrada \\
\hline$n$ & número máximo de clusters que podem ser formados \\
\hline$b_{i j}$ & pesos bottom-up \\
\hline$t_{j}$ & pesus top-down \\
\hline$\rho$ & parâmctro de vigilância \\
\hline $\mathbf{s}$ & vetor de entradas binárias \\
\hline $\begin{array}{l}\mathbf{x} \\
\|\mathbf{x}\|\end{array}$ & $\begin{array}{l}\text { vetor de at.jvação de } F_{1(b)} \text { > bivárivo } \\
\text { norma do vetor } \mathbf{x}\end{array}$ \\
\hline
\end{tabular}

Tabela 3.2: Notação utilizada no algoritmo de aprendizado da rede ART1.

\section{$\diamond$ Algoritmo de treinamento da rede ART1}

A Figura 3.2 pode ajudar a entender o algoritmo de treinamento da rede ART1. Para melhor compreensão deste algoritmo, a notação apresentada na Tabela 3.2 será usada.

\section{Parâmetros usados no algoritmo de treinamento da rede ART1}

A Tabela 3.3 mostra como os parâmetros e pesos da rede podem ser pré-definidos pelo usuário e suas respectivals restriçòes (Carpenter \& Grossberg 1987b).

\begin{tabular}{ll}
\hline \hline Parâmetros & Restriçóes \\
\hline$L$ & $L>1$ \\
$\rho$ & $0<\rho \leqslant 1$ \\
$b_{i j}$ & $0<b_{i j}(0)<\frac{L}{L-1+m}$ \\
$l_{j i}$ & $t_{j i}(0)=1$ \\
\hline
\end{tabular}

Tabela 3.3: Inicializaçâo dos parâmetros da rede ART1.

\section{$\diamond$ Resumo do algoritmo da rede ART1}

O algoritmo de treinamento apresentado aqui é o de modo rápido.

$$
\text { t) }
$$

- Inicialização: deternine o tamanho das camadas $F_{1}$ e $F_{2}$, e os valores para os demais parâmetros da rede, sendo que para cada vetor de entrada, é preciso ajustar toda unidade de $F_{2}$ para zero, ou seja. $y_{j}=0$, para todo $j$ :

- Entrada: calcule $\|\mathbf{s}\|$ c para todos os elementos de $F_{1(b)}$ faça $x_{i}=s_{i}$. 
- Busca: calcule a ativação de cada neurônio da camada $F_{2}$ com $y_{j}=\sum_{i} b_{i j} x_{i}$ e encontre o neurônio $J$ com a maior ativação:

$$
y J=\max \left\{y_{j}, j=1 \ldots n\right\} .
$$

Se houver mais que um y. então utilize o menor índice $j$, mantendo-se a ordem de $j=1,2,3 \ldots n$. Caso todos os $y_{j}$ sejam iguais a -1 , significa que os neurônios estão inibidos, e aquele padrão de entrada não será aprendido durante aquele ciclo de treinamento.

- Comparação (ou Resct): calcule o sinal de ativação das unidades de $F_{1(b)} \operatorname{com} x_{i}=s_{i} t_{. J}$, calcule a norma $\mid x \|$ e teste a unidade de Reset com:

$$
\frac{\|\mathbf{x}\|}{\|\mathbf{s}\|}>\rho
$$

Se a desigualdade for verdadeira, então vá para o próximo estágio. Caso contrário, faça $y_{J}=-1$ (i.c.. o ncurônio ficará inibido durante a apresentação daquele padrāo), sendo necessário que se repita o estágio de Busca;

- Adaptação: atualize os pesos da rede de acordo com as seguintes regras:

$$
\left\{\begin{array}{l}
b_{i J}^{(\text {Nowo })}=\frac{L x_{i}}{L-1+\|\mathbf{x}\|}, \\
t_{J i}^{(\text {Nowo })}=x_{i} .
\end{array}\right.
$$

\section{Fuzzy ART}

O modelo Fuzzy ART incorpora todas as caracteristicas básicas dos sistemas ART (Carpenter \& Grossberg 1987b). Assim como o modelos ART1, apresentado na seção anterior, este modelo trabalha com o paradigma de aprendizado näo supervisionado. Além disso, este modelo ART foi desenvolvido bascardo-se no modelo ART1, mas adaptado ao tratamento de valores reais pertencertes ao intervalo [0, 1] (i.e, valores fuzzy) (Carpenter, Grossberg \& Rosen 1991b). Mais detalhes a respcito destc modelo também podem ser encontrados nos trabalhos de (Frank, Kraiss \& Kuhlen 1999) e (Baraldi \& Blonda 1999). 


\section{$\diamond$ Arquitetura da rede Fuzzy ART}

Os componentes da arquitetura da rede Fuzzy ART são: a camada $F_{1}$ (dividida cm duas sub-camadas, de entrada e de interface, respectivamente, $F_{1(a)}$ e $F_{1(b)}$ ); a camada $F_{2}$ (camada de clusters); a unidade de Reset o o conjunto de pesos, representado pela matriz $\mathbf{W}$, que conecta a sub-camada $F_{1(b)}$ com a canada $F_{2}$. Entre as unidades da sub-camada $F_{1(a)}$ e as unidades da sub-camada $F_{1(b)}$ ocorre o pré-processamento dos dados de entrada. As unidades da sub-camada $F_{1}(b)$ também estào conectadas con a unidade de Reset. A arquitetura completa deste modelo pode ser vista na Figura 3.3.

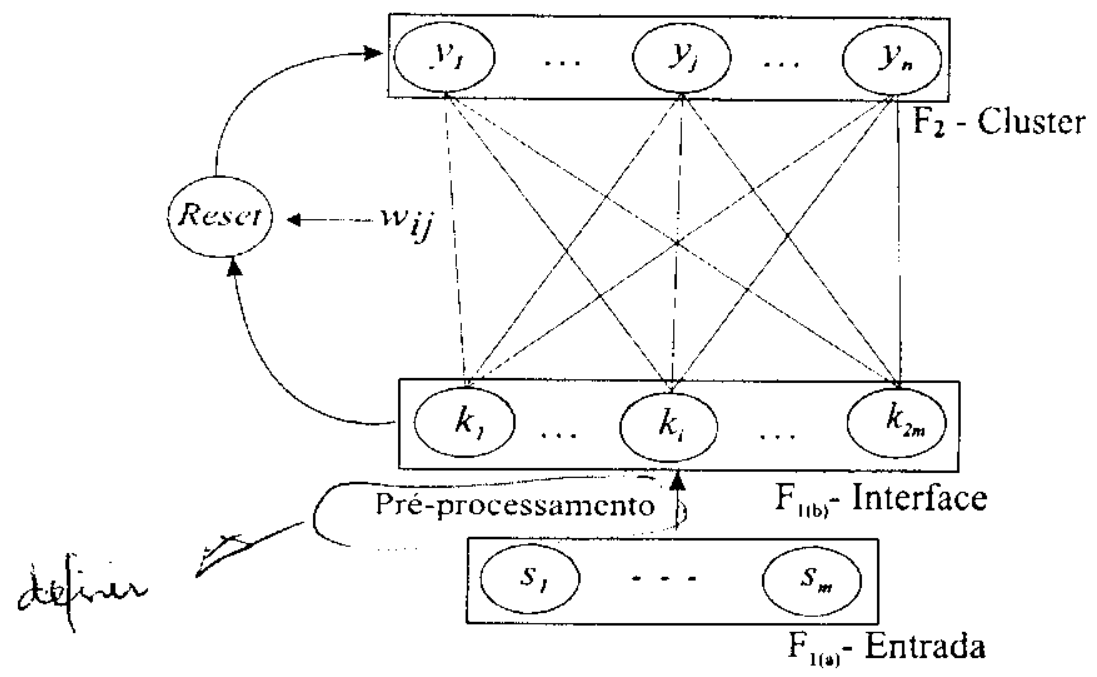

Figura 3.3: Arquitetura da rede Fuzzy ART.

\section{$\diamond$ Descrição do treinamento da rede Fuzzy ART}

O processo de treinamento da rede Fuzzy ART pode ser descrito como segue. O sinal de cntrada migra da sub-cannada $F_{1(u)}$ para a sub-camada $F_{1(b)}$, após sofrer um pré-processamento. Em seguida, o sinal atinge a camada $F_{2}$, reforçado pelo valor dos pesos contidos nas conexões, e isto determina as ativaçōes em todas as unidades da camada $F_{2}$. Imediatamente após o estágio de busca, determina-se o neurónio com a maior ativaşão. $O$ algoritmo segue avaliando se o neuronio vencedor, chl $F_{2}$. c suficientemente similar ao padrão de entrada $\mathbf{x}$; se for verdadeiro, a rede entra em processo de atualização de pesos (ressonância). 


\begin{tabular}{|c|c|}
\hline Parâmetros & Descrição \\
\hline$m$ & Dinensão do vetor de entrada. \\
\hline$n$ & Núnero máximo de clusters que podem ser formados. \\
\hline$\varepsilon$ & $\begin{array}{l}\text { Parametro de escolha. Nos experimentos realizados neste } \\
\text { presente trabalho, foi utilizado um valor } \varepsilon \approx 0 \text {. }\end{array}$ \\
\hline$\beta$ & $\begin{array}{l}\text { Taxa de aprendizagem. Tem scus valores no intervalo }[0,1] \text {. } \\
\text { Valor } 1 \text { detcrmina treinamento no modo rápido; valor } \beta<1 \\
\text { determina treinamento no modo intermediário. }\end{array}$ \\
\hline $\bar{\rho}$ & $\begin{array}{l}\text { Parametro de vigilância. Determina a similaridade entre } \\
\text { padroes em unn mesmo cluster. }\end{array}$ \\
\hline $\bar{W}$ & $\begin{array}{l}\text { Representia a matriz de pesos. Neste modelo, os pesos top- } \\
\text { down e bottom-up são iguais. Portanto, apenas um destes } \\
\text { conjuntos ć usado. }\end{array}$ \\
\hline
\end{tabular}

Tialuela 3.4: Parâmetros da rede Fuzzy ART.

\section{$\diamond$ Resumo do algoritmo da rede Fuzzy ART}

No inicio do treinamento os valores da matriz de pesos $\mathbf{W}$ são iguais a 1 e cada categoria, ou neurônio da canlada $F_{2}$. deve ser iniciado na condição de não comprometido. Esta situação será modificada. para comprometido, somente se o neurônio for selecionado para o estágio de Adaptação.

- Inicialização: detcrmine o tamanho das camadas $F_{1}$ e $F_{2}$, bem como os valores para os demais parâmetros cla rede. Além disso, para cada vetor de entrada, é preciso ajustar todas as unidades de $F_{2}$ para zero, ou seja, $y_{j}=0$, para todo $j$;

- Entrada: normalize o vetor de entradas para que seus valores se encontrem no intervalo $(0,1)$. O vetor resultante dessa operação é denotado por $\boldsymbol{\nu}$.

Enl seguida, transforme cada vetor resultante $\nu m$-dimensiona em um vetor $2 m$-dimensional $\left(k_{1} \ldots, k_{2 m}\right)$. Isto é feito calculando-se o código complementar como nà Equaçāo (3.20). Este cálculo é usado para evitar perda de informação e proliferação de clusters em $F_{2}$ (Carpenter, Grossberg \& Rosen 1991c), (Frank, Kraiss \& Kuhlen 1999). Dessa forma, o vetor obtido após o estrág̣io de pré-processamento, é dado por:

$$
\mathbf{k}=\left(\nu, \nu^{c}\right)=\left(\nu_{1}, \ldots, \nu_{n}, 1-\nu_{n}, \ldots, 1-\nu_{n}\right) .
$$

- Busca: para cadia vetor de entrada, calcule sua ativação $y_{j}$ com: 


$$
y_{j}=\frac{\left\|\mathbf{k} \wedge \mathbf{w}_{j}\right\|_{2}}{\varepsilon+\left\|\mathbf{w}_{j}\right\|_{2}}
$$

sendo $\|\cdot\|_{2}$ a norma definida por:

$$
\|\mathbf{u}\|_{2}=\sum_{i=1}^{2 n} u_{i},
$$

que é a soma dos compomentes do vetor $\mathbf{u}$. O operador $\wedge$ ć um opcrador fuzzy de conjunção (ou $A N D$ ). e isto significa que:

$$
a \wedge b \equiv \min (a, b)
$$

ou seja, o menor valor entre $a$ e $b$. Então para $\mathbf{k}$ e $\mathbf{w}_{\mathbf{j}}$, tem-se que:

$$
\mathbf{k} \wedge \mathbf{w}_{\mathbf{j}}=\left(k_{1} \wedge u_{j}, \ldots, k_{\mathbf{z}} \wedge w_{z j}\right), \quad z=2 m
$$

O neurônio vencedor é indexado por $J$. Para encontrar este índice é necessário calcular a maior ativação de $y_{j}$ èn $F_{2}$, que é o maior valor de $y_{j}$ considerando a Equação (3.21), ou seja.

$$
y=\max \left(y_{j}\right), \quad j=1 \ldots n \text {. }
$$

Se houver mais que: $u$ m $y_{J}$. então utilize o menor índice $j$, mantendo-se a ordem de $j=1,2, \ldots, n$. Caso todos os $y_{j}$ sejam jguais a -1 , significa que os neuronios estão inibidos, e aquele padrão dọ contrada não scrá aprendido durante aquele ciclo de treinamento.

- Comparação: após tcr selecionado o índice do neurônio com o maior $y_{J}$, e se este estiver comprometido, chtão testa-se:

$$
y_{J}>\rho
$$

Se a desigualdade for verdadeira, então vá para o próximo estágio. Caso contrário, faça $y_{J}=-1$ (i.e., o neurônio ficará inibido durante a apresentaçāo daquele padrão), sendo necessário que se repita o cstágio de Busca; 
- Adaptaçāo: atualize os pesos dat rede de acordo com a seguinte regra:

$$
\mathbf{w}_{J}^{(\text {No'o })}=\beta\left(\mathbf{x} \wedge \mathbf{w}_{J}^{(\text {Antigo })}\right)+(1-\beta) \mathbf{w}_{J}^{(\text {Antigo })}
$$

\section{Consideraçōes sobre Alguns Modelos ART}

Em (Vicentini 2002) e (Vicentini, Oliveira \& Romero 2004), foi realizada uma avaliaçāo para encontrar qual seria, dentre os modelos ART2, ART2-A e Fuzzy ART, o mais adequado para ser combinado com a estrutura de dados métrica Slim-Tree (Traina Jr., J. M. Traina, Seeger \& Faloutsos 2000). Nesse trabalho, foi proposto um modelo híbrido para indexação e recuperação de informaçòes: que fosse capaz de responder a consultas por similaridade. Para realizar a análise comparativa cntre os três modelos $\Lambda R T$ foi escolhida a tarefa de classificação de dados provenientes do algumas bases disponíveis no repositório UCI (Blake \& Mer\% 1998) e do conjunto Eigenfaces (Wactlar, Kanade, Smith \&. Stevens 1996), que pertence ao projeto Informedia, da Universidade Carnegic Mellon, nos Estados Unidos. Como resultado desses experinentos, obscrvou-se que os desempenhos dos modelos ART2 e ART2-A foram similares. considerando-sf as matrizes de confusāo obtidas pelos mesmos. Por outro lado, os resultados obtidos pela rede Fuzzy ART náo conseguiram superar aqueles obtidos pelos dois outros modelos. Con base nesta avaliação. o modelo que aprescntou melhores resultados foi o modelo ART2-A.

Existem ainda, na literatura, outros trabalhos dedicados ao estudo e análise de modclos ART. Mais especificamente sobre a rede Fuzzy ART, em (Frank, Kraiss \& Kuhlen 1999) e (Baraldi \& Blonda 1999). são apontadas algumas limitações do modelo, que podem ser resumidas nos seguintes ítens:

- A rede ó dependente dia orton de contrada dos padrōes de treinamento;

- A rede é altamente sensivel a ruídos no conjunto de dados de treinamento;

- Em experimentos com clados de alta dimensão, foi observado um certo grau de incoerência no mapeamento dos clusters.

Em estudos preliminares realizados no decorrer do presente trabalho, os modelos ART2A e Fuzzy ART, implementados em (Vicentini 2002), foram aplicados para a segmentação de imagens. Neste caso, foi observado, já nos primeiros experimentos envolvendo esse tipo de aplicaçāo, que a rede neulal Fuzzy ART teve um desempenho superior ao modelo ART2-A.

Porém, com o intuito de obter resultados ainda melhores, uma vez que a rede Fuzzy ART apresenta sensibilidade a ruídos, foi proposta neste trabalho, uma metodologia para pré- 
processamento de imagens que combina uma técnica baseada em ICA para redução de ruídos e o operador de Sobcl para deteção de bordas, que será apresentada no Capítulo 5. A utilização desta técnica para pré-processiar imagens antes de submetê-las à rede, de fato, acarretou em melhorias observadas nos resultados finais de segmentação, como pode ser visto em (Oliveira, Vicentin \& Ronero 2002) e no Capítulo 8 deste presente trabalho.

\section{Aplicaçōes das redes ART}

De forma geral, os modelos ART podem ser aplicados a problemas que envolvem aprendizado, reconhecimento a prcvisão. Dentre as aplicaçōes das redes ART de paradigma não supervisionado e as de paradigma supervisionado, pode-se citar: controle motor sensorial de robôs (Bachelder, Waxman \& Scibert 1993); navegação de robôs (Racz \& Dubrawski 1995); visão computacional (Caudell \& Healy 1994); reconbecimento de objetos 3D (Scibert \& Waxman 1992): reconhecimesto (le face (Seibert \& Waxman 1993); reconhecimento de ondas de eletrocardiograma (Han \& Han 1996) (Suzuki 1995); reconhecimento automático de alvos (Bernardon \& Carrick 1995) (Waxman, Scibert, Gove, Fay, Bernardon, Lazott, Stecle \& Cunningham 1995) (Moya, Fioch \& Howtetler 1993); previsão da estrutura secundária de proteínas (Mehta, Vij \& Rabelo 1993): reconbecimento de caracteres chineses (Gan \& Lua 1992) e segmentação de imagens (Soliz \& Donohoe 1996), (Nakamma \& Ogasawara 1999), (Oliveira. Vicentin \& Romero 2002).

\subsubsection{Rede Neural PCA Adaptativa}

Nesta seção, é apresent arda a rede PCA Adaptativa, uma outra rede neural auto-organizável que. por sua vez, implementa o aprendizado não supervisionado hebbiano (ver Seção (3.3.1)). Este modelo, que incorpora a técnica PCA clássica (ver Seção 3.2.1), foi proposto por (Rubner \& Tavan 1989) e sua ar(quit etura ć ilustrada pela Figura 3.4. Esta consiste de $p$ neurônios de entrada e $m$ neurônios de saicha. $\mathrm{cm}$ que cada unidade de entrada $i$ cstá conectada a cada neurônio de saída $j$, com peso de comexia $u_{i j}$.

As unidades de sajia dessa rede são organizadas de forma que o neurônio de saída $i$ é conectado ao neurônio de saída $j$ por meio da conexão com peso $u_{i j}$ se, e somente se, $i<j$.

A saída $y_{j}(n)$ do neurônio $i$ no tempo $n$, produzida em resposta ao conjunto de entradas $\left\{x_{i}(n) \mid i=0,1, \ldots, p-1\right\}$ ć dada por:

$$
y_{j}(n)=\sum_{i=0}^{p \cdots 1} u_{\eta_{j}}(n) x_{i}(n)+\sum_{l<j} u_{l j}(n) y_{l}, \quad j=0,1, \ldots, m-1
$$




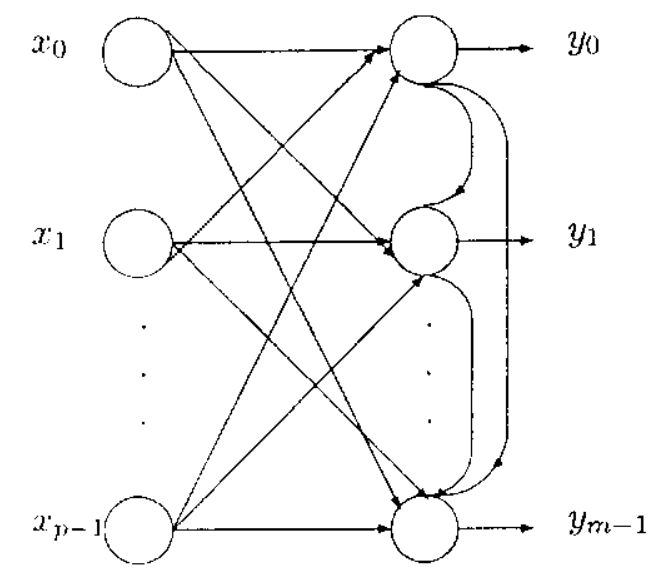

Figura 3.4: Rede Neural PCA Adaptativa.

Os pesos das conexões $w_{i j}$ entre a camada de entrada e a camada de saída são ajustados de acordo com a regra de aprendizado de Hebb, ou seja,

$$
\Delta u_{i j}(n)=\eta r_{i}(n) y_{j}(n), \quad i=0,1, \ldots, p-1 ; \quad j=0,1, \ldots, m-1,
$$

em que $\eta$ é o parâmetro de velocidade de aprendizado.

Os pesos sinápticos latcrais, por sua vez, são ajustados de acordo com a regra antihebbiana, isto é,

$$
\Delta u_{l j}(n)=-\mu y_{l}(n) y_{j}(n), \quad l<j
$$

em que $\mu$ é outro parâmetro de aprendizagem positivo.

Devido à regra de aprendizado anti-hebbiana, a tendência dos pesos laterais ć diminuir assintoticamente. Por esse motivo. quando esses pesos sinápticos tiverem atingido um valor suficientemente pequelio, a rede terá convergido para um estado no qual os $m$ vetores de pesos associados às $m$ uridades cle saídas são os $m$ autovetores da matriz de correlação dos dados de entrada, com autovalomes $\lambda_{13}>\lambda_{2}>\cdots>\lambda_{m-1}$.

Pode-se acelerar a convergência da rede, introduzindo um termo momentum $\beta$ c deixando que os parâmetros de aprendizagem e o momentum diminuam com o tempo. Em (Mao \& Jain 1995), utitizou-se:

$$
\begin{gathered}
\Delta u_{i j}(n+1)=\eta(n) x_{i} y_{j}+\beta(n) \Delta w_{i j}(n) \\
\Delta u_{l j}(n+1)=-\mu(n) y_{l} y_{j}+\beta(n) \Delta u_{l j}(n),
\end{gathered}
$$

em que $\eta(n+1)=\max (\alpha \eta(n), 0.0001), \mu(n+1)=\max (\alpha \mu(n), 0.0002), \beta(n+1)=\max (\alpha \beta(n), 0.0001)$ 
e $\alpha$ é o fator de diminuiçào.

O seguinte teorema de convergência para essa rede foi proposto e provado por (Sanger 1989):

"Se a matriz de pesos sinápticos $\mathbf{W}(n)$ for associada a valores aleatórios no tempo $n=0$, cntão, com probabilidlade 1. a regra generalizada de Hebb irá convergir na média, e $\mathbf{W}^{T}(n)$, no limite, irá aproximar-se de uma matriz cujas colunas serāo os primeiros $m$ autovetores da matriz C de correlaçāo dos vetores de entrada $\mathrm{x}(n)$, ordenados por ordem decrescente de autovalor." Portanto, no limite. pode-se escrever:

$$
\Delta \mathrm{w}_{j}(n) \rightarrow 0 \quad \text { e } \quad \mathbf{w}_{j} \rightarrow \mathbf{a}_{j}, \quad j=0,1, \ldots, m-1,
$$

tal que $\left\|\mathbf{w}_{j}(n)\right\|=1$, para todo $j$. Os valores $\mathbf{a}_{0}, \mathbf{a}_{1}, \ldots, \mathbf{a}_{m-1}$ representam os autovetores normalizados associados aos $m$ maiores autovalores da matriz $\mathbf{C}$ de correlação dos vetores de entrada $\mathbf{x}(n)$, estando esses autoralores ordenados em ordem decrescente.

As Tabelas 3.5 × 3.6 apresentam os autovetores encontrados pelas técnicas PCA clássica e rede PCA Adaptativa para o ben conhecido conjunto de dados Iris, apresentado com mais detalhes no Capítulo 8. Pode-se notar, portanto, que de fato a rede PCA Adaptativa consegue aproximar os resultados obtidos pela técnica clássica.

Tabela 3.5: Resultaclos da PCA clássica para o conjunto de dados Iris.

\begin{tabular}{|c|c|c|c|c|}
\hline \multirow[b]{2}{*}{ Componente } & \multicolumn{4}{|c|}{ Autovetores (coeficientes) } \\
\hline & $x_{1}$ & $\mathbf{x}_{2}$ & $\mathrm{x}_{3}$ & $\mathrm{x}_{3}$ \\
\hline 1 & 0.522371 & -0.263356 & 0.581254 & 0.565611 \\
\hline 2 & 0.372320 & 0.925556 & 0.021094 & 0.065417 \\
\hline 3 & -0.721015 & 0.242033 & 0.140889 & 0.633804 \\
\hline 4 & -0.261998 & 0.124137 & 0.801155 & -0.523543 \\
\hline
\end{tabular}

Tabela 3.6: Resultados da rede PCA Adaptativa para o conjunto de dados Iris.

\begin{tabular}{|c|c|c|c|c|}
\hline \multirow[b]{2}{*}{ Componente } & \multicolumn{4}{|c|}{ Autovetores (coeficientes) } \\
\hline & $x_{1}$ & $\mathrm{x}_{2}$ & $\mathbf{x}_{3}$ & $x_{1}$ \\
\hline 1 & 0.522249 & -0.269216 & 0.580671 & 0.563560 \\
\hline 2 & 0.383352 & 0.920919 & 0.026521 & 0.065160 \\
\hline 3 & -0.723120 & 0.238417 & 0.141955 & 0.632538 \\
\hline 4 & -0.269590 & 0.118567 & 0.800430 & -0.524957 \\
\hline
\end{tabular}




\section{Aplicaçōes da PCA em Análise de Imagens}

A técnica PCA tem sido bastante utilizada em aplicações na área de análise de imagens, como no reconhecimento do fares humanas (Moghaddam \& Pentland 1996), (Martínez 2000), reconhecimento de objetos (Murase \& Nayar 1995), aplicações de robótica industrial (Nayar, Nene \& Murase 1995) e robótica móvel (Weng 1996).

Em experimentos preliminares realizados neste trabalho (Oliveira, Romero, Mazucheli \& Nonato 2000). foi avaliado o desempenho da rede PCA Adaptativa, apresentada na seçāo 3.3.3, na tarefa de compressào de um conjunto de imagens médicas composto por 208 imagens com dimensão 480x640. nas quais cada pixel é representado por 8 bits contendo o seu correspondente valor de nivel de cinza. Tais imagens representam cortes de um fígado humano e foram tomadas a partir de um microscópio laser, pclo Departamento de Patologia da Fiocruz (Fundação Oswaldo Cruz - Rio de Janeiro).

Foi rcalizado, nesse trabalho. um estudo comparativo entre os desempenhos da Rede PCA Adaptativa, da PCA clássica e do padrão JPEG ${ }^{4}$ (Nelson 1992), que é o mais utilizado para a compressão de imagens de vários tipos. Medindo-se os Erros Quadráticos Médios (MSE) ${ }^{5}$ entre as imagens originais c as recuperadas, para taxas de compressão de $93 \%$ e $96 \%$ (aproximadanente), pode-se notar que, de fato, os resultados obtidos pela rede PCA Adaptativa aproximam-se daqueles atingidos pela abordagem clássica, sendo, ainda, melhores do que os resultados conseguidos através da aplicação do método JPEG, como pode ser visto na Tabela 3.7 .

Una das vantagens da abordagem que utiliza a rede neural PCA é que este método não necessita calcular a matriz de covariância e seus respectivos autovalores e autovetores. Computando-se somente operaçōes de soma e multiplicações de matrizes, consegue-se atingir resultados bem próximos dos encontrados pela PCA clássica.

Com o objetivo de comprovar a habilidade da rede neural para aprender e adaptar-se aos dados a esta apresentados, foram realizados alguns testes, considerando as imagens do conjunto como uma sequêncial $e$ aproveitando o aprendizado obtido pela compressão de uma imagem para a compressão da imagem subsequente. Os resultados desse teste mostraram que, de fato, o tempo necessário para a convergĉncia da Rede PCA Adaptativa diminui à medida que esta é inicializada com os pesos utilizados para o treinamento da imagem anterior.

Além disso, foi obscrvado que a compressão JPEG (ver Figura 3.5 (c)) fez com que as imagens recuperadas apresentassem alguns padrōes, o que dificulta a análise da imagem

\footnotetext{
${ }^{4}$ do original, em ingles, Joint Plsotugraphic Expert Group.

${ }^{5}$ do original, ern ingles. Mean-Squarc Error.
} 
e a visualizaça de pequenos objetos. Já nas imagens recuperadas pelas duas metodologias que implementam a PCA, (ver Figuras 3.5 (d) e (e)) a maioria dessas pequenas estruturas é preservalia, o que é inportante para anátises de imagens de fígado, uma vez que a presença desses pequenos objetos pode indicar algum tipo de doença.

Tabela 3.7: Resultados do compressão para o JPEG, PCA clásicica e rede PCA.

\begin{tabular}{|c|c|c|c|c|c|c|}
\hline & \multicolumn{6}{|c|}{ MSE } \\
\hline & \multicolumn{2}{|c|}{ J]FC } & \multicolumn{2}{|c|}{ Rede PCA } & \multicolumn{2}{|c|}{ PCA Clásica } \\
\hline & $96 \%$ & $93 \%$ & $96.875 \%$ & $93.75 \%$ & $96.875 \%$ & $9.3 .75 \%$ \\
\hline Imatron 1 & 9.72 & 7.01 & 5.82 & 4.91 & 5.43 & 4.69 \\
\hline lmiagen 2 & 9.97 & 7.28 & 6.75 & 5.49 & 6.17 & 5.17 \\
\hline ImLizesn 3 & 8.91 & 6.44 & 5.20 & 4.08 & 4.64 & 3.77 \\
\hline Imasem 1 & 9.30 & 6.72 & 5.80 & 4.36 & 5.18 & 4.07 \\
\hline Inagem & 9.47 & 6.76 & 6.08 & 1.50 & 5.30 & 4.16 \\
\hline Imiagenes 6 & 8.90 & 6.14 & 5.19 & 4.00 & 4.64 & 3.75 \\
\hline Tringem 7 & 90.1 & 6.51 & 5.41 & 3.965 & 4.81 & 3.74 \\
\hline Innagem 8 & 8.99 & 6.48 & 5.39 & 4.06 & 4.79 & 3.81 \\
\hline Imarem 0 & 8.93 & 6.40 & 5.32 & 3.92 & 4.66 & 3.68 \\
\hline Janatgenn 10 & 9.47 & 6.63 & 5.61 & 4.22 & 5.01 & 3.97 \\
\hline
\end{tabular}

\subsection{Consideraçōes Finais}

Neste capítulo. form apresentadas, primeiramente, duas técnicas de análise multivariada, denominadas P'CA e técnicals de agrupanentos (Clustering). A compreensão da técnica PCA é de findamental importuncia para o cntendimento do método ICA, que será apresentado no próximo capítulo. Dessa forma, foram feitas consideraçós a respeito dos fundamentos e procedimentos relacionados à PCA. A lém disso, foi descrito como esse método pode ser aplicado para reduzir a dimensionalidacle de um conjunto de dados.

A outra técnica de análise multivariada discutida neste capítulo diz respeito a técnicas de agrupamentos (Clustering). () algoritmo k-médias implementa um método auto-organizável básico para cncontrar agrupamentos cm um conjunto de dados. Fsse algoritmo também foi apresentado neste capítulo e será utilizado nas análises comparativas realizadas no Capítulo 8.

Além disso, form ressittakles alguns fundamentos e paradigmas de aprenclizado ineren-

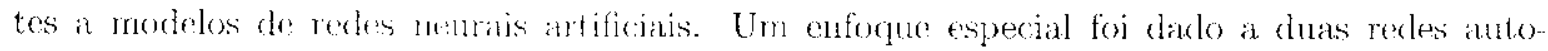




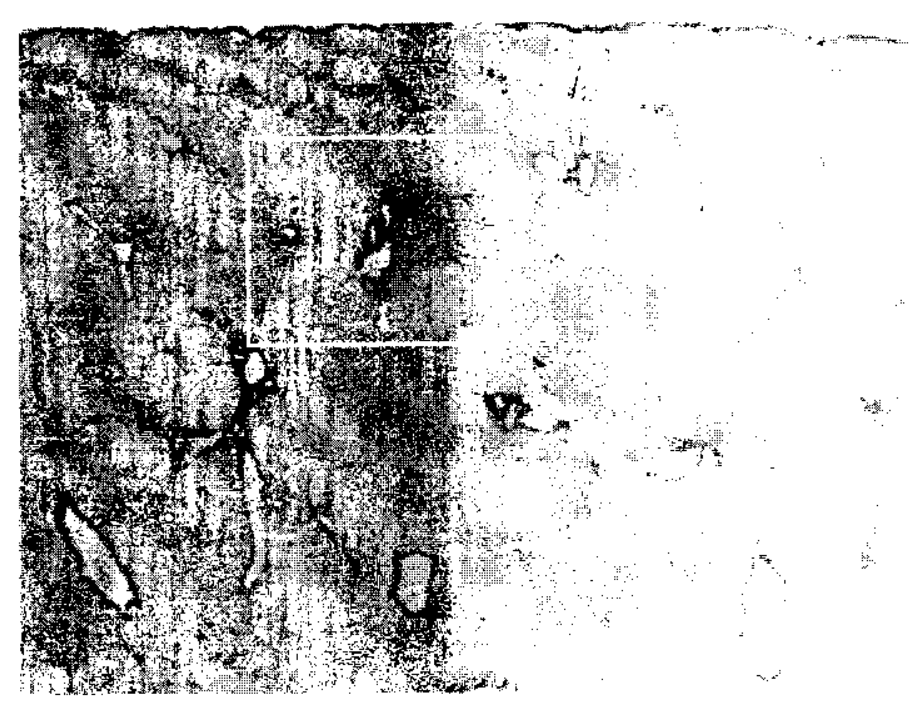

(a)

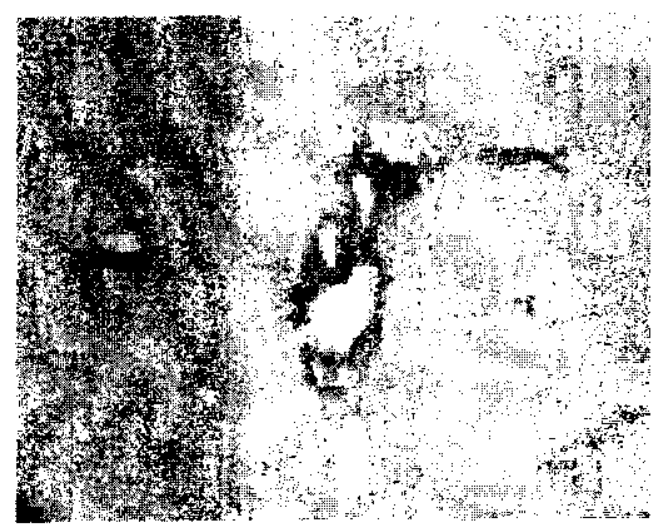

(b)

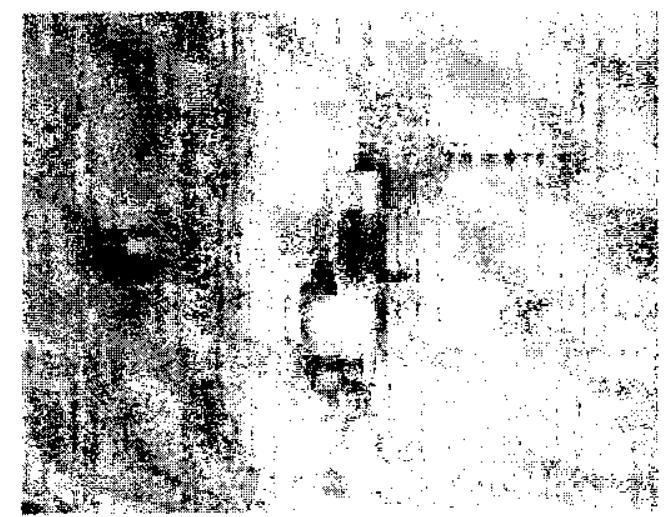

(d)

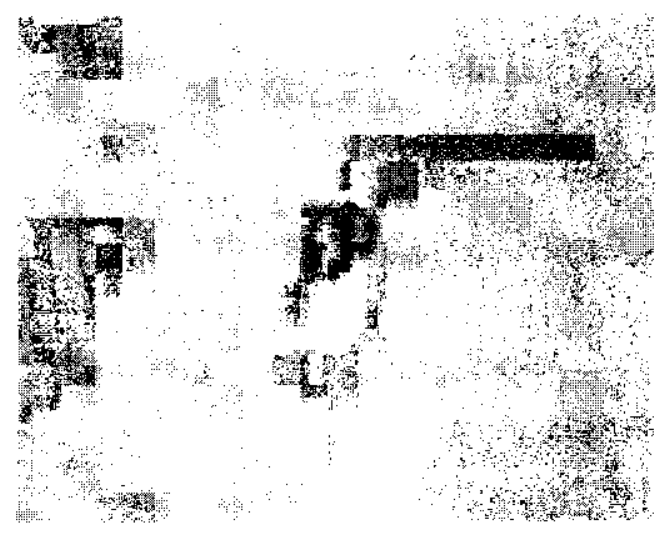

(c)
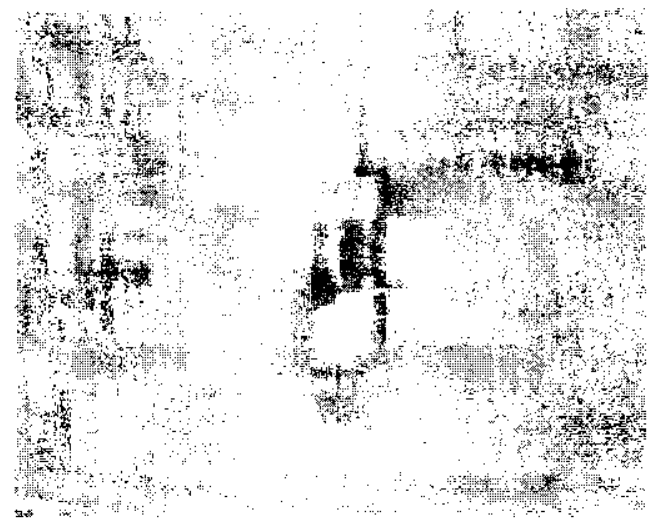

(e)

Figura 3.5: Resultados obtidos pelas técnicas de compressio. (a) imagem original, (b) uma

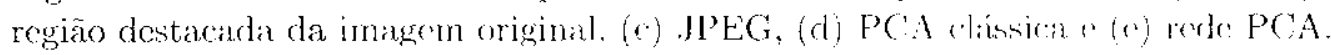


organizáveis que implementam as técnicas PCA e de agrupamento. O primeiro modelo descrito refere-se à rede neural Fuzzy ART, que utiliza um algoritmo de aprendizado competitivo $\mathrm{e}$ incremental. Tal modelo integra conceitos da Teoria da Ressonância Adaptativa e fundamentos da lógica nebulosa ( fuzzy), tendo sido aplicada nos experimentos para segmentação de imagens apresentados no Capitulo 8. Todavia, em estudos encontrados na literatura, verificou-se que este modelo de rede nenral ó sensível a ruídos, dentre outras limitações também apontadas neste capítulo.

Com o intuito de amenizar os efeitos causados pela presença de ruídos nos resultados de segmentação obtidus pela rede Fuzzy ART, foi proposta no presente trabalho uma metodologia de pré-processamento que scrá descrita no Capítulo 5 e que combina uma técrica para redução de ruídos baseada em ICA e o operador de Sobel para deteç̧ão de arestas. Os resultados da utilização dessa metodologia aplicada a tarefas de segmentação pela rede Fuzzy ART podem ser vistos no Capítulo 8.

O outro modelo de rede neural auto-organizável apresentado neste capítulo diz respeito à rede neural PCA Adaptativa. Esse modelo foi aplicado para encontrar os autovetores do conjunto de dados Iris e os resultados desses experimentos demonstraram como a rede PCA pode de fato aproximar os resultados do método estatístico clássico. Essa rede também foi utilizada em cxperimentos com imagens ao longo do presente trabalho. Neste caso, os resultados obtidos por essa rede nà tarefia de (compressão/descompressão de imagens médicas superaram os resultados obtidos polo padrão de compressão JPEG. 


\section{Capítulo 4}

\section{Análise de Componentes Independentes}

\subsection{Considerações Iniciais}

Nos últinuss anos, a técnicia da Análise de Componentes Independentes (ICA) tem sido bastante aplicada en várias árcas da ciência, devido ao fato de que esse método explora estatísticas de ordem mais altas em um conjunto de dados (Hyvärinen, Karhunen \& Oja 2001), (Comon 1994), (Bell de Sejnowski 1995). Como scrá visto neste capítulo, o método ICA tem sido utilizado com sucesso na análise de dados, processamento de sirnais e modelagem de funções cerebrais relacionadas a visaro (Bell \& Sejnowski 1997).

De fato, a técnica ICA é uma generalização da Análise de Componentes Principais (PCA), uma vez que o método ICA transforma linearmente as variáveis originais em componentes independentes, ao invés de somente não correlacionadas, como no caso da PCA.

Neste capítulo, descreve-se o método ICA, juntamente com as suas aplicações, suposiçōes, restrições e ambiguidades. Alén disso. são apresentadas várias abordagens para realização da ICA e as relaçós entre as mesulas.

O entendimento da ICA é de fundamental importância para a compreensão do método Sparse Code Shrinkage para redução de ruídos, que será apresentado no Capítulo 5 e do modelo ICAMM para classificaçào não supervisionada, descrito no Capítulo 6. 


\subsection{Motivação}

Uma motivação bastante utilizada para ilustrar o funcionamento do método ICA ć considerar uma situação na qual existem vários sinais emitidos por fontes ou objetos físicos diferentes (Hyvärinen, Karhumen \& Oja 2001). Tais fontes poderiam ser, por exemplo, diferentes áreas do cérebro emitindo sinais clétricos; pessoas conversando em um ambiente e emitindo, portanto, sinais de fala: ou tclcfones móvcis cmitindo ondas de rádio. Deve-se assumir, ainda, que existcm vários sensores ou recejtores. Esses sensores estāo em diferentes posições, de modo que cada sinal registrado é uma soma pondcrada (isto ć, uma combinação linear) dos diferentes sinais de fontes originais.

Como exemplo, pode-se inaginar a seguinte situação: dois intcrlocutores estão conversando em um anbiente no qual estào instalados dois microfones em diferentes localizações. Os microfones 1 e 2 fornecem sinais registrados em instantes de tempo e que poderiam ser denotados por $x_{1}(t)$ e $x_{2}(t)$. respectivamente. Neste caso. $x_{1}$ e $x_{2}$ são as amplitudes do sinal e $t$ o instante de tempo. Cada um desses sinais registrados é uma soma ponderada dos sinais de fala $s_{1}(t)$ e $s_{2}(t)$ emitidos pelos dois interlocutores. Dessa formá, os sinais $x_{1}(t)$ e $x_{2}(t)$ podem ser expressos pelas seguintes equaçóes lincires:

$$
\begin{aligned}
& x_{1}(t)=a_{11} s_{1}(t)+a_{12} s_{2}(t) \\
& x_{2}(t)=a_{21} s_{1}(t)+a_{22} s_{2}(t),
\end{aligned}
$$

onde $a_{11}, a_{12}, a_{21}$ e $a_{22}$ sân alguns parâmetros que dependem das distâncias entre os microfones (scnsores) c os interlocutores (fontes). Assume-se que esses parâmetros são desconhecidos, uma vez que nāo é possível saber o valor de $a_{i j}(i, j=1,2)$, sem conhecer todas as propriedades do sistema físico, o que. cm geral. ć uma tarefa difícil. O objetivo é, nesse caso, estimar os dois sinais originais $s_{1}(t)$ e $s_{2}(t)$, usindo somente os sinais registrados $x_{1}(t)$ e $x_{2}(t)$. Esse problema específico é referenciado na itteratura como problema do cocktail party, ou, de forma mais geral, problema da separação cega de sinais de fontes (BSS) ${ }^{1}$.

Para ilustrar esse problema, pode-se considerar as formas de ondas das Figuras 4.1 e 4.2 (Hyvärinen, Karhunen \& Oja 2001), apesar destas não corresponderem a sinais realistas. Os sinais originais $s_{1}(t)$ e $s_{2}(t)$ poderiam se apresentar como na Figura 4.1 e os sinais misturados $x_{1}(t)$ e $x_{2}(t)$ poderiam ser como aqueles vistos na Figura 4.2. O problema resume-se, então, a recuperar os dados da. Figura 4.1 usando somente os dados presentes na Figura 4.2.

\footnotetext{
${ }^{1}$ do original, em ingles. Blind Source Separation.
} 


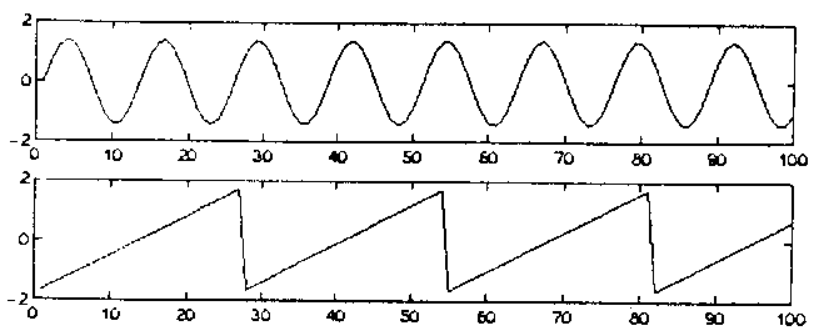

Figura 4.1: Exemplo de sinais originais.

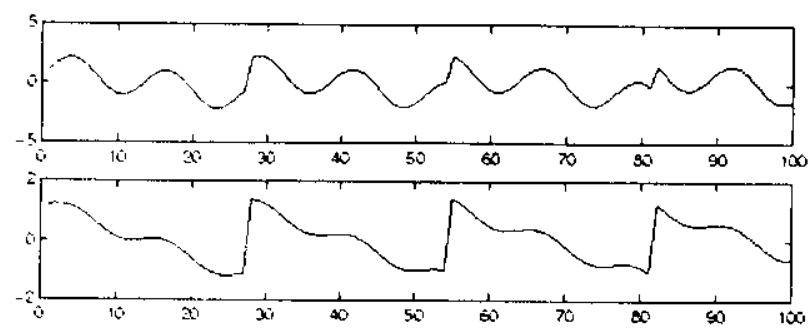

Figura 4.2: Fxemplos de misturas de sinais observadas.

Uma abordagem para solucionar esse problema seria utilizar alguma informação sobre as propriedades estatísticas dos sinais $s_{i}(t)$ para estimar os parâmetros $a_{i j}$. No caso da ICA, suficiente assumir que $s_{1}(t)$ e $s_{2}(t)$. em qualqucr instante $t$, são estatisticamente independentes. Essa suposição nāo é realistia em muitos casos, e não é necessário que seja exatantente verdadeira na prática (Hyvärinen. Karhunen \& Oja 2001). A técnica ICA pode ser utilizada para estimar os parâmetros $a_{i j}$, baseando-se na informação de independência, o que permite separar os dois sinais de fontes originais $s_{1}(t)$ e $s_{2}(t)$ a partir de suas misturas $x_{1}(t)$ e $x_{2}(t)$. A Figura 4.3 (Hyvärinen, Karhunen \& Oja 2001) fornece os dois sinais estimados pelo método ICA. Como pode ser visto, estes sinais estào muito próximos dos sinais de fontes originais (a inversão dos sinais nāo é importante nesse caso).

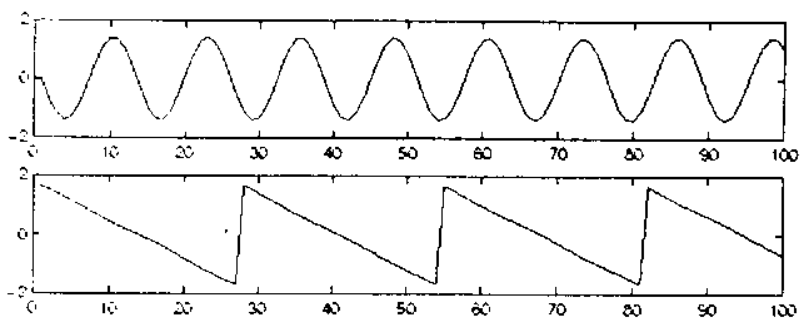

Figura 4.3: As entimativas dos sinais de fontes originais, calculadas usando somente os sinais observados na Figura 4.2. 


\subsection{ICA em Algumas Aplicaçōes Reais}

Uma aplicação real importante da ICA diz respeito à análise de registros de atividades cerebrais, como por exemplo. registros dados por eletroencefalograma (EEG) e magnetoenccfalograma (MEG). Esses dados consistem $\mathrm{cm}$ registros de potenciais elétricos ou magnéticos medidos em diferentes localizacioes do escalpo ${ }^{2}$ on do cérebro. Neste caso, pode-se assumir que os dados são gerados pela mistura de componentes relativas a atividades cerebrais e musculares. Essa situação é bem similar ao problema do cocktail party: deseja-se encontrar as componentes originais da atividade ccrebral, mas somente é possivel observar as misturas de componentes. A ICA pode, então, revelar informações interessantes sobre a atividade cerebral, utilizando as componentes independentes cucontradas pelo método. Dentre vários trabalhos relevantes envolvendo ICA e esse tipo de aplicação, pode-se citar (Makeig, Jung, Bell, Ghahramani \& Sejnowski 1997), (Vigário, Särelï. Jousmäki. Mämäläinen \& Oja 2000) e (Jung, Makeig, McKeown, Bell, Lee \& Sejnowski 2001).

Também nas ciências sociais, a ICA tem sido considerada uma ferramenta importante para a descoberta de fatores independentes, por exemplo, em problemas de ecomometria (Back \& Weigend 1997), (Oja, Kiviluoto \& Mālāroiu 2000).

Uima outra aplicaçăo importante da ICA refere-se à utilizaçāo desta técnica como método para extração de caractcrísticas relcvantes em um conjunto de dados. As características encontradas nesse tipo de aplicação da ICA podem ser usadas para representar imagens (Bell \& Sejnowski 1997), (Hyvärinen, Oja, Hoyer \& Hurri 1998), (Olshausen \& Field 1996), dados de áudio (Bell \& Sejnowski 1996 ) e outros tipos de dados em tarefas como compressão e supressão de ruídos.

\subsection{Definição da Técnica ICA}

Para definir rigorosamente a técnica ICA (Comon 1994), (Jutten \& Herault 1991), pode-se utilizar o modelo estatístico de variáveis latentes, que são variáveis que não podem ser diretamente observadas. Assume-se que são observadas $n$ variáveis aleatórias $x_{1}, x_{2}, \ldots, x_{n}$ modeladas como combinações linèures de $n$ variáveis aleatórias latentes $s_{1}, s_{2}, \ldots, s_{n}$ :

$$
x_{i}=a_{i 1} s_{1}+a_{i 2} s_{2}+\ldots+a_{i n} s_{n}, \quad \text { para todo } i=1, \ldots, n
$$

onde $a_{i, j}, i, j=1, \ldots, n$ são alguns coeficientes reais. Por definição, as variáveis $s_{i}$ são estatisti-

\footnotetext{
${ }^{2}$ Pele que cobre o tope da cabeca humana.
} 
camente mutuamente independentes.

O modelo ICA ́. portanto, um modelo generativo, isto é, que descreve como os dados são gerados por um processo de mistura das variáveis $s_{j}$, denominadas de componentes independentes. Os únicos termos observáveis no modelo ICA são as variáveis aleatórias $x_{i}$, uma vez que as variáveis $s_{j}$ são latentes e us cocficientes $a_{i j}$ são desconhecidos. Portanto, tanto as componentes independentes $s_{j}$ guanto os coeficientes $a_{i j}$ devem ser estimados utilizando somente os valores observados das variáveis alnatórias $x_{i}$.

$O$ índice relativo ao instante de tempo $t$ foi omitido na Equação(4.3), uma vez que, no modelo ICA, assume-se que cada mistura $x_{i}$, assim como cada componente independente $s_{j}$, săo variáveis alcatórias, deixando de ser consideradas como sinais $\mathrm{cm}$ um determinado instante de tempo.

Utilizando a notaçāo votorial. denota-se por $\mathrm{x}$ o vetor aleatório cujos elementos são as combinaçócs lincares $x_{1}, x_{2} \ldots \ldots x_{n}$, e por $\mathbf{s}$ o vetor aleatório com os elementos $s_{1}, s_{2}, \ldots, s_{n} \mathrm{e}$ por A a matriz com os corficiontes $a_{t, j}$. Nesse caso, convenciona-se que todos os vetores do modelo são vetores-columa: portanto $\mathbf{x}^{T}$, o vetor transposto de $\mathbf{x}$, é um vetor-linha.

Dessa forma. na notracia vetorial, o modelo ICA pode ser escrito como:

$$
\mathbf{x}=\mathbf{A s}
$$

ou, de forma equivalente.

$$
\mathbf{x}=\sum_{i=1}^{n} \mathbf{a}_{i} s_{i}
$$

onde os vetores $\mathbf{a}_{i}$ sian ats colmuats da matriz $\mathbf{A}$.

No decorrer deste trabatho, assume-se que o número de vetores observados é igual ao número de sinais de fontcs, embora em várias aplicações seja mais realístico assumir que esses números sejam difermtes (ver. por exemplo, (Olshausen \& Field 1997), (Lewicki \& Scjnowski $2000)\rangle$

\subsection{Suposições e Restriçōes da ICA}

Para assegurar que o modelo ICA básico apresentado na Seção(4.4) possa ser estimado, algumas suposiçōes e restrições, enumeradas a seguir, são necessárias.

1. Deve-se assumir gue as componentes indepondentes $s_{j}$ são estatisticamente independentes. Esse princípio é a laase da ICA. 
2. As componentes independentes devem apresentar distribuições não gaussianas.

Os cumulantes de alta ordem são iguais a zero para distribuiçōes gaussianas, porém informações de ordem mais alta são essenciais para a estimaçāo do modelo ICA (ver seções 7.4 e 7.5 em (Hyvärinen, Karhunen \& Oja 2001)). Portanto, o procedimento para a técnica ICA fica praticamente impossível se as variáveis observadas tiverem distribuições gaussianas.

Uma medida de não nomalidade frequentemente utilizada na estimação ICA é a medida de kurtosis, dada pela seguinte equação:

$$
\text { רurt }(y)=E\left\{y^{4}\right\}-3
$$

onde y é uma variável aleatória. Uma variável gaussiana apresenta um valor de kurtosis igual a zero. Variávejs com kurtosis positivas possuem uma distribuiçăo supergaussiana, como é o caso da distribuiçāo de Laplace (ou dupla exponencial). Já um valor de kurtosis negativo inplica em unia distribuiçāo subgaussiana, como no caso da distribuição uniforme. Exemplos dessas distribuiçoes podem ser vistos na Figura (4.4).

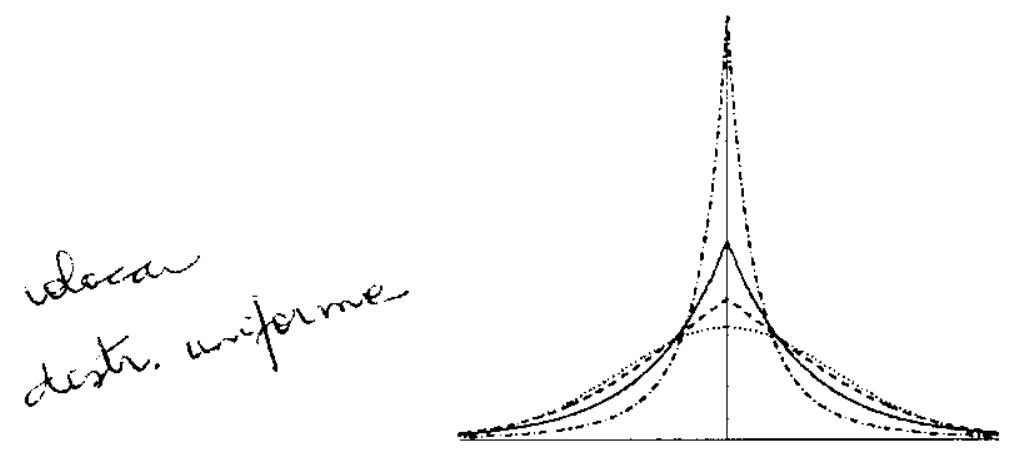

Figura 4.4: Exemplos de funçós de densidade de probabilidade supergaussianas e subgaussianas. Linha sollida: densidade Laplaciana. Linha tracejada: densidade moderadamente supergaussiana. Linha pontilhada-traccjada: densidade fortemente supergaussiana. Linha pontilhada: densidade gaussiana.

3. Para simplificar o modelo, pode-se assumir que a matriz de coeficientes A é quadrada.

Ern outras palavras, o número de componentes independentes é igual ao número de variávcis observadas. Fmbora, em alguns casos, essa suposição possa ser relaxada (ver, por cxemplo, (Olshausen \& Fiold 1997), (Lewicki \& Sejnowski 2000)), é muito importante considerá-la, uma vez que a mesma simplifica bastante o modelo. Partindo dessa suposiçāo, e 
também assumindo que $\mathbf{A}$ é inversível, depois de estimar a matriz $\mathbf{A}$, é possível computar a sua inversa, $\mathbf{W}$, c obter as componentes independentes por meio de:

$$
\mathrm{s}=\mathbf{W} \mathbf{x} .
$$

Dessa forma, também deve-se assumir que a matriz de coeficientes $\mathbf{A}$ é inversível.

4. Sem perda de generalidade, pode-se assumir que as variáveis observadas e as componentes independentes possuem média igual a zero. Essa suposiçāo é feita pois simplifica bastante a teoria cos algoritmos derivados da técnica ICA.

Se essa suposição não for verdadeira, pode-se proceder com uma etapa de pré-processamento para ICA. na qual as variáveis observadas são subtraídas da sua média amostral. Isso significa que as valriáveis originais, denotadas aqui por $\mathbf{x}^{\prime}$ podem ser pré-processadas utilizandose a seguinte equaçio:

$$
\mathbf{x}=\mathbf{x}^{\prime}-E\left\{\mathbf{x}^{\prime}\right\}
$$

Dessa forma as componentes independentes também terão média zero, una vez que:

$$
E\{\mathrm{~s}\}=\mathbf{A}^{-1} E\{\mathbf{x}\}
$$

\subsection{Ambiguidades do Modelo ICA}

Analisando o modelo ICA formulado na Equação (4.4), pode-se notar que as seguintes ambiguidades ou indeterminancias precisam ser consideradas:

1. Nào é possivel determinar as variâncias das componentes independentes.

A razão para essa afirmação reside no fato que, uma vez que s e A são desconhecidos, qualquer multiplicador escalar $\alpha_{i}$ aplicado a uma das fontes $s_{i}$ pode ser cancelado dividindo-se a coluna correspondente $\mathbf{a}_{i}$ de A pelo mesmo escalar:

$$
\mathbf{x}=\sum_{i}\left(\frac{1}{\alpha_{i}} \mathbf{a}_{i}\right)\left(s_{i} \alpha_{i}\right)
$$

Procedendo dessa forma, as magnitudes das componentes independentes podem scr facilmente restauradas. Felizmente, essa ambiguidade é insignificante na maioria das aplicações. 
2. Não é possível determinar a ordem das componentes independentes, tal como acontece na técnica PCA, na qual as componentes estão ordenadas pelas suas variâncias.

Novamente devido ao fato de que s e $\mathbf{A}$ são desconhecidos, a ordem dos termos da somatória na Equação (4.5) pode mudar livremente, considerando qualquer uma das componentes independentes como sendo a primeira.

\subsection{Não Correlação, Branqueamento e Independência}

Dadas algumas variávcis alcatórias, é possível utilizar métodos lincares para transformálas em variáveis nào correlacionadas. Um processo utilizado para esse fim é denominado de branqueamento (whitening) e pode ser implementado utilizando-se a técnica PCA. No entanto, métodos de decorrelação de varíveis, como a PCA, não podem ser utilizados para encontrar as componentes independentes en un conjunto de dados, uma vez que não correlação não implica cm independência. como discutido no Capítulo 2.

O proccsso de branqueamento corresponde a transformar linearmente o vetor de dados observados $\mathbf{x}$, multiplicando-o por alguma matriz $\mathbf{V}$ :

$$
\mathrm{z}=\mathrm{Vx}
$$

de modo a obter um nowo velor $\mathbf{z}$ que seja branco.

Uma ve\% que a operaçào de branqueamento consiste essencialmente em uma operação de decorrelação segnida de uma mudança de escala, a técnica PCA pode ser usada para esse propósito.

Seja $\mathbf{E}=\left(\mathbf{e}_{1} \ldots \mathbf{e}_{n_{i}}\right)$ a matriz cujas colunas são os autovctores com norma unitária da matriz de covariância $\mathbf{C}_{\mathbf{x}}=E\left\{\mathbf{x x}^{T}\right\}$. Esses autovetores podem ser computados a partir de uma amostra dos vetores $\mathrm{x}$ por moio de métodos estatísticos clássicos (ver, por excmplo, (Johnson \& Wichern 1998)): ou utilizando algum algoritmo de aprendizagem para PCA (ver, por exemplo, (Diamantaras \& Kung 1996) e (Oliveira 1997)).

Seja $\mathbf{D}=\operatorname{diag}\left(d_{1} \ldots d_{n}\right)$ a matriz diagonal com os autovalores de $\mathbf{C}_{\mathbf{x}}$. Então uma transformaça linear de branqueamento pode ser dada por:

$$
\mathbf{V}=\mathbf{D}^{-1 / 2} \mathbf{E}^{T}
$$

uma vez que os elementos de $\mathrm{D}$ correspondem às variâncias das componentes originais $\mathrm{x} e$ os vetores de $\mathrm{E}$ forneccm as bases para o novo subespaço no qual as componentes sāo não 
correlacionadas.

Além do operador lincar $\mathbf{V}$ da Equação(4.12), qualquer matriz UV, com $\mathbf{U}$ sendo uma matriz ortogonal, tambćm é uma matriz de branquemento. Uma instância importante de UV é a matriz $\mathbf{E D}^{-1 / 2} \mathbf{E}^{T}$, que foi obtida multiplicando-se a matriz $\mathbf{V}$ da Equação(4.12), pela esquerda, pela matriz ortogonal E. A matriz resultante dessa operação é a matriz inversa da raiz quadrada de $\mathrm{C}_{\mathbf{x}}$, denotada por $\mathrm{C}_{\mathbf{x}}^{-1 / 2}$, o que simplifica os cálculos envolvidos no processo de branqueamento.

\subsection{Abordagem ICA baseada na Teoria da Informação}

Nos trabalhos pioneiros sobre componentes independentes, o estudo da separaçāo de sinais de fontes misturados e observados em um vetor de sinais de sensores era considerado um problema clássico de processannento de sinais de dificil solução. Num trabalho importante de separação cega de fontes (BSS). Herault e Jutten (Herault \& Jutten 1986) introduziram um algoritmo adaptativo $\mathrm{cm}$ mma arquitetura simples, com feedback, capaz de separar várias fontes independentes desconhecidas. Essa abordagem foi posteriormente pesquisada por Jutten e Herault (Jutten \& Herault 1991). Karhunen c Joutsensalo (Karhunen \& Joutsensalo 1994) e Cichocki et al. (Cichocki, Unbehauen \& Rummert 1994). Comon (Comon 1994) introduziu o conceito de Análise de Componentes Independentes e propôs funçôes de custo relacionadas à aproximação da minimizaçăo da informação mútua entre os sensores.

Fim paralelo aos est ndos envolvendo o problema de BSS, regras de aprendizado não supervisionado baseadas na teoria dia informação foram propostas por Linsker (Linsker 1992). A meta cra maximizar a informaçio mútua entre as entradas e saídas de uma rede neural. Essa abordagem está relacionada ao princípio da redução de redundância sugerido por Barlow (Barlow 1961) como uma estratégia para codificação em neurônios. De acordo com esse princípio, cada neurônio deveria codificar características que fossem tão estatisticamente independentes dos outros neurônios quanto possivel. considerando um determinado conjunto de entradas. Roth e Baram (Roth \& Baram 1996) e Bell e Sejnowski (Bell \& Sejnowski 1995) derivaram, independentemente, regras de aprendizado por gradiente estocásticas para essa maximização e aplicaram-nas, respectivamente. à previsāo e análise de séries temporais e à separação cega de fontes. Bell e Sejnowski (Bell \& Sejnowski 1995) incorporaram o problema BSS a um arcabouço de teoria da informação e demonstraram a eficiencia do modelo para a scparação de fontes misturadas. Seus métodos sian mas plausiveis a partir de uma perspectiva de processamento neural do que as funções de custo baseadas em cumulantes proposta por Comoll (Comon 1994). Um 
método adaptativo para BSS, similar a esse trabalho de Comon, foi proposto por Cardoso e Laheld (Cardoso 1998).

Outros algoritmos para realizar ICA foram propostos a partir de diferentes pontos de vista. Abordagens de Estimação por Máxima Verossimilhança (MLE) ${ }^{3}$ para ICA foram inicialmente propostos por Gaeta e Lacoume (Gaeta \& Lacoume 1990) e elaborados por Pearlmutter e Parra (Pearlmutter \&: Parra 1996). Algoritmos de PCA não linear, que foram desenvolvidos por Karhunen e Joutsensalo (Karhmen \& Joutsensalo 1994), Xu (Xu 1993) e Oja (Oja 1997), também podem ser vistos a jartir do princípio de maximizaçāo da informação (também chamado de infomax), uma vez que estes algoritmos aproximadamente minimizam a informação mútua nas saídas da rede.

A seguir, será demonstrado como uma abordagem para ICA pode ser formulada em um arcabouço para o problema de separação de fontes, baseando-se em conceitos da teoria da informação.

\subsubsection{Formulação do Problema ICA Utilizando Conceitos de Teoria de In- formação}

Inicialmente assume-sc que existe um vetor $M$-dimensional com média zero $\mathrm{s}(t)=$ $\left[s_{1}(t), \ldots, s_{M}(t)\right]^{T}$, cujos componentes são mutuamente independentes. O vetor $\mathbf{s}(t)$ corresponde aus $M$ sinais de fontes independentes $s_{i}(t)$ com valores escalares. Dessa forma, pode-se escrever a fdp multivariada do vetor $s(t)$ como o produto das distribuiçöes marginais independentes:

$$
p(\mathbf{s}(t))=\prod_{i=1}^{M} p_{i}\left(s_{i}(t)\right) .
$$

Um vetor de dados $\mathbf{x}(t)=\left[x_{1}(t), \ldots, x_{N}(t)\right]^{T}$ ć observado a cada instante de tempo $t$, tal que:

$$
\mathbf{x}(t)=\mathrm{As}(t)
$$

onde A ć uma matriz escalar $N \times M$. Como as componentes $x_{i}$ dos vetores observados não são consideradas como sendo independentes, a fdp multivariada $p(\mathbf{x})$ não irá satisfazer a igualdade de produto na Equação (4.13). A informação mútua $I(x)$ do vetor observado é dada pela medida de divergência $D(. \|$.) de Kullback-Leibler (KL) entre a densidade multivariada $p(\mathbf{x})$ e a densidacle escrita na forma de produto:

\footnotetext{
${ }^{3}$ do original, em ingles. Maximum Likclihood Estimation.
} 


$$
I(\mathbf{x})=D\left(p(\mathbf{x}) \| \prod_{i=1}^{N} p_{i}\left(x_{i}\right)\right)=\int p(\mathbf{x}) \log \frac{p(\mathbf{x})}{\prod_{i=1}^{N} p_{i}\left(x_{i}\right)} d \mathbf{x} .
$$

A informação mútua é sempre positiva e é igual a zero somente quando as componentes $x_{i}$ sâo independentes (Cover \& Thomas 1991). Portanto, nessa abordagem, a informação mútua dos dados observados é utilizada como uma medida de dependência a ser minimizada.

A meta da ICA ć encontrar uma transformação linear $\mathbf{W}$ dos sinais de sensores dependentes $\mathbf{x}$ que torne as saidas $\mathbf{u}$ tâdo independentes quanto possível:

$$
\mathbf{u}_{t}=\mathbf{W} \mathbf{x}_{t}=\mathbf{W A s}_{t}
$$

onde $\mathbf{u}$ é uma estimativa das fontes originais. As fontes são recuperadas de forma exata quando $\mathbf{W}$ é a inversa de $\mathbf{A}$. Todavia, existem ambiguidades nesse problema (ver Seção (4.6)) e a matriz $\mathrm{A}^{-1}$ geralmente nào poxle ser detcrminada na sua forma exata. O que pode ser feito é estimar uma versão rescalonada e permutada dos $M$ sinais de fontes, uma vez que não é possível conhecer a magnitude e a ordem clesseses simais.

Para a estimação da matriz $\mathbf{W}$ pela abordagem infomax, além das suposiçôes e restrições da ICA apresentadas na Seçào(4.5), deve-se supor a ausência de ruídos de sensores, ou no máximo a existência de sinais com poncos ruidos aditivos. Essa suposiçào é necessária para satisfazer a condição de maximizaçato de informação, que estabelece que a informação mút.ua entre as saídas somente é minimizada no caso de pouco ruído (Linsker 1992), (Nadal \& Parga 1994).

\subsubsection{Abordagem Infomax para ICA}

Considerando um processador neural com entradas $x$ e saídas $y$, Nadal e Parga (Nadal \& Parga 1994) mostrauan que a maximização da transferência de informaçāo em uma rede neural não linear mininiza a informaçào mútua entre as saídas quando a otimização é aplicada aos pesos sinápticos W e à função de transferência não linear $g(\mathbf{u})$. Roth e Baram (Roth \& Baram 1996) c Bell c Scjnowski (Bcll \& Sejnowski 1995) derivaram, independentemente, regras de aprendizado por gradiente para resolver esse problema de maximização, e aplicarann-nas, respectivamente, para previsão o análise de sćrics temporais e separação cega de fontes. Bell e Sejnowski (Bell \& Sejnowski 1995) propuseram um algoritmo de aprendizado simples para una rede neural feedforward que separa cegamente misturas lineares $\mathrm{x}$ de fontes independentes s, utilizando o princípio de maximização de informação. Eles mostraram que maximizar a entropia conjunta $H(\mathbf{y})$ da saída de um processador neural pode aproximadamentc minimizar a informação mútua entre as componentes de saída $y_{i}=g\left(u_{i}\right)$, onde $g\left(u_{i}\right)$ é uma não linearidade 
monotônica inversivel, ou seja, possui uma única função inversa $g^{-1}\left(u_{i}\right)$, e $\mathbf{u}=\mathbf{W} \mathbf{x}$.

A entropia conjunta das saídas de uma rede neural é dada por:

$$
H\left(u_{1} \ldots, y_{N}\right)=H\left(y_{1}\right)+\ldots+H\left(y_{N}\right)-I\left(y_{1}, \ldots, y_{N}\right)
$$

onde $H\left(y_{i}\right)$ são as entropias marginais das saídas e $I\left(y_{1}, \ldots, y_{N}\right)$ é a informaçāo mútua dessas saídas. Maximizar $H\left(y_{1} \ldots y_{N}\right)$ consiste, portanto, em maximizar as entropias marginais e minimizar a informação mútua $\int(\mathbf{y})$.

Esse problema é, ainda, equivalente à maximizar a informação mútua $I(\mathbf{y}, \mathbf{x})$ que as saídas y de uma rede neural possuem sobre as suas entradas $\mathbf{x}$. Essa medida pode ser definida como:

$$
I(\mathbf{y}, \mathbf{x})=H(\mathbf{y})-H(\mathbf{y} \mid \mathbf{x})
$$

onde $H(\mathbf{y} \mid \mathbf{x})$ ć a entropia da saŕla que não tenha advindo da entradas $\mathbf{x}$. No caso de ausencia de ruído (ou melhor, quando nào se sabe o que é ruído e o que é sinal na entrada), o mapcamento cntre $\mathbf{x}$ e $\mathbf{y}$ ć determinístico c $I(\mathbf{y} \mid \mathbf{x})$ possui um valor muito pequeno, podendo, portanto, ser desprezado no processo de maximização.

As saídas y são variáveis aleatórias de amplitude limitada e, portanto, as entropias marginais $H\left(y_{i}\right)$ atingem scus valores máximos quando as distribuições de $y_{i}$ forem uniformes. Maxjmizar a entropia conjunta também irá diminuir $I\left(y_{1}, \ldots, y_{N}\right)$, una vez que a informação mútua é semple positiva. Para $I\left(y_{1}, \ldots, y_{N}\right)=0$, a entropia conjunta é a soma das entropias marginais:

$$
H\left(y_{1}, \ldots y_{N}\right)=H\left(y_{1}\right)+\ldots+H\left(y_{N}\right)
$$

Dessa forma. o valor máximo para $H\left(y_{1}, \ldots, y_{N}\right)$ é atingido quando a informação mútua entre as variáveis aleatórias $\not 1 \ldots . . . y_{N}$ for igual a zero e suas distribuições marginais forem uniformes.

Nessa abordagem. os pesos sinápticos $\mathbf{W}$ são determinados maximizando-se a entropia conjunta em relação à W. Nêsses caso, a derivada da Equação (4.17) em relação à $W$ pode ser escrita em termos da divergência KL entre a distribuiçāo uniforme multivariada, denotada por $p_{1}(\mathbf{y})$, e a estimativa da distribuiçào multivariada $p(\mathbf{y})$ :

$$
\frac{\partial H(\mathbf{y})}{\partial \mathbf{W}}=\frac{\partial}{\partial \mathbf{W}}\left(-D\left(p_{\mathbf{1}}(\mathbf{y}) \| p(\mathbf{y})\right)\right) .
$$

No limite, quando a função de transferência $g\left(u_{i}\right)$ e W são otimizados, a entropia conjunta $H(\mathbf{y})$ ć máxima, c $p(\mathbf{y})=p_{1}(\mathbf{y})$, de modo que $I(\mathbf{y})=0$. Sc $g\left(u_{i}\right)$ for um mapeamento inversível de $u_{i}$ para $y_{i}$, a divergência $\mathrm{KL}$ nł Equação(4.20) é igual à divergência KL entre as estimativas da 
distribuição de fontes $p(\mathbf{u})$ e da distribuiçāo das fontes verdadeiras $p(\mathbf{s})$,

$$
D\left(p_{\mathbf{1}}(\mathbf{y}) \| p(\mathbf{y})\right)=D(p(\mathbf{s}) \| p(\mathbf{u}))
$$

uma vez que a divcrgência KL é invariante para qualquer transformação inversível.

Se a informação mútua entre as saídas for igual à zero, ou scja, $I\left(y_{1}, \ldots, y_{N}\right)=0$, a informaçào mútua antes de aplicar a não linearidade, $I\left(u_{1}, \ldots, u_{N}\right)$, também deve ser igual à zero. Isso ocorre porque a funçăo não linear $g\left(u_{i}\right)$ não introduz quaisquer dependências. Unı resultado fundamental encontrado na literatura estabelece que, quando $g\left(u_{i}\right)$ é monotonicamente crescente ou decrescente (isto ć. possui uma única funçāo inversa $g^{-1}\left(u_{i}\right)$ ), a relação entre $u_{i} \mathrm{e}$ $y_{i}$ é dada por (Papoulis 1991):

$$
p\left(y_{i}\right)=\frac{p\left(u_{2}\right)}{\left|\frac{\partial y_{i}}{\partial u_{i}}\right|}=\frac{p\left(u_{i}\right)}{\left|\frac{\partial g\left(u_{2}\right)}{\partial u_{2}}\right|} .
$$

Se $y_{i}$ apresentar una distribuiçầo nuiforme, segue que:

$$
p\left(u_{i}\right)=\left|\frac{\partial g\left(u_{i}\right)}{\partial u_{i}}\right|
$$

uma vez que, nesse caso. $p\left(y_{i}\right)$ atinge o seu valor máximo igual a 1.

Isso significa que $u_{i}$ é uma variável independente com uma distribuição que possui aproximadamente a forma dà derivada da não lincaridade, $g$. No caso da função logística, (ver Figura(4.5a)), a fdp apropriada possui a forma apresentada na Figura(4.5b). Bell e Sejnowski (Bell \& Sejnowski 1995) ralizaram a separaçāo de misturas de vários sinais de fala e música utilizando a abordagen infomax com funçăo de transferência logística, também chamada de funçäo sigmóide.

Uma arquitetura simples que pode realizar o mapeamento de $\mathbf{x}$ para $\mathbf{y}$ consiste em uma rede neural feedforward com uma única camada e uma função de ativação de saída não linear $y_{\imath}=g\left(u_{i}\right)$. A escolha clessa nào linearidade é esscncial para que a minimização da informação mútua realize a técnica ICA. Cono proposto em (Bell \& Sejnowski 1995) e apresentado a seguir, as regras de aprendizacio para ICA podem scr derivadas por meio da maximização da entropia de saída $H(y)$ de un processador neural. 
Funçào Logística

a)

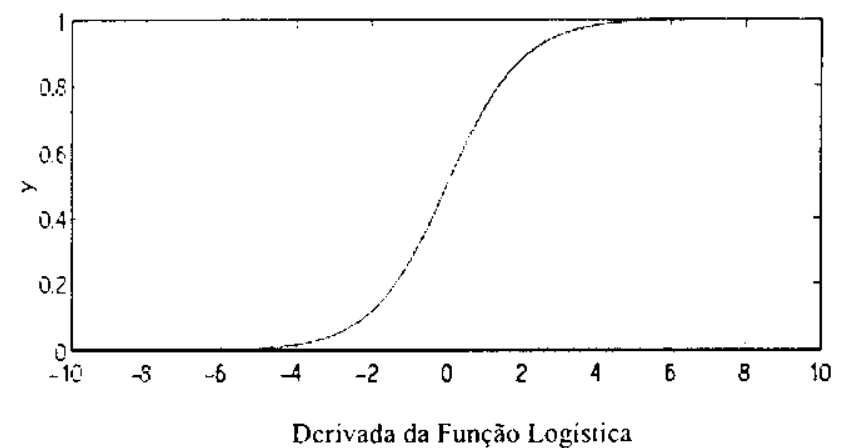

b)

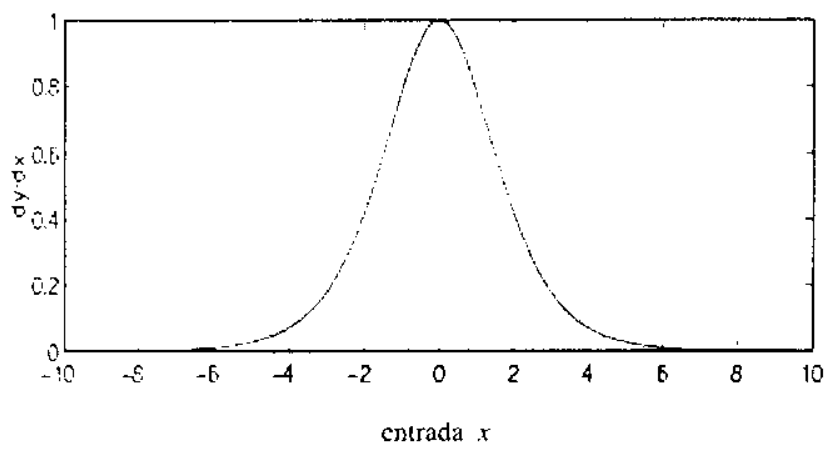

Figura 4.5: (a) A funçà logística $(y=1 /(1+\exp (-x)))$ e (b) sua derivada $\left(\frac{\partial y}{\partial x}=y(1-y)\right)$.

\subsubsection{Regras de Aprendizado ICA para uma Rede Neural com uma Entrada e uma Saída}

Quando uma linica entrada $x$ passa por uma função de transferência $g(x)$ para resultar em uma variável de saída $y$, a entropia da saída, $H(y)$ é maximizada, alinhando-se as partes de alta densidade da fdp de $x$ com as partes de alta inclinação da função $g(x)$ (ver, como ilustração, a Figura(4.5)).

Adaptando a Equaçàn(1.22) para esse caso, tem-se que:

$$
p_{y}(y)=\frac{p_{x}(x)}{\left|\frac{\partial y}{\partial x}\right|}
$$

A entropia da saída di dada por:

$$
H(y)=-E\left\{\ln p_{y}(y)\right\}
$$

Substituindo at Fquacăo(4.24) na Equação(4.25), tem-se que: 


$$
H(y)=E\left\{\ln \left|\frac{\partial y}{\partial x}\right|\right\}-E\left\{\ln p_{x}(x)\right\} .
$$

O segundo termo à direita dà Equação(4.26) refere-se à entropia de $x$, podendo-se, portanto, considerar que o mesmo năo é afetado pelas alterações no parâmetro $w$ que determinam $g(x)$. Dessa forma, para maximizar a entropia de $y$, por meio de mudanças aplicadas à $w$, é necessário concentrar-se apenas na maximização do primeiro termo da Equação(4.26), que corresponde ao valor esperado do logaritmo do quanto a entrada $x$ afeta a saída $y$. Isso pode ser realizado considerando-se un conjunto de treinamento de entradas $x$ para aproximar a densidade $p_{x}(x)$, e derivando-se uma regra de aprendizado por subida de gradiente, da seguinte forma:

$$
\Delta w^{\prime} \times \frac{\partial H}{\partial u}=\frac{\partial}{\partial u}\left(\ln \left|\frac{\partial y}{\partial x}\right|\right)=\left(\frac{\partial y}{\partial x}\right)^{-1} \frac{\partial}{\partial w}\left(\frac{\partial y}{\partial x}\right)
$$

No caso da função logística,

$$
\begin{aligned}
& y=\frac{1}{1+e^{-u}}, \quad u=w x+w_{0} \\
& y^{\prime}=\frac{\partial y}{\partial u}=y(1-y)
\end{aligned}
$$

e as regras de aprendizado derivadas a partir da fórmula geral dada na Equação(4.27) são as seguintes:

$$
\begin{array}{ll}
\Delta w: & \frac{1}{w}+x(1-2 y) \\
\Delta w_{0} \times 1-2 y .
\end{array}
$$

As derivaçoes completas para as regras nas Equações (4.30) e (4.31) podem ser encontradas no Apèndice A.

A Figura(4.6) ilustrá o efeito das regrats para atualização de $w \mathrm{e} w_{0}$. Por exemplo, se a fdp da entrada. $p_{x}(x)$ for gaussiana, a regra para $w_{0}$ irá alinhar a parte mais inclinada da curva sigmóide com o pico de $p_{x}(x)$. casando a densidade da entrada com a curva da função $y$. Esse método para aproximar densidades foi inicialmente proposto em (Roth \& Baram 1996). A regra para $w$ irá, dessa forma, escalonar a inclinaçāo da curva sigmóide para estimar a variância de $p_{x}(x)$. Por exernplo: uma fdp apresentando uma forma estreita iria resultar cm uma sigmóide cle alta inclinaşàò. 


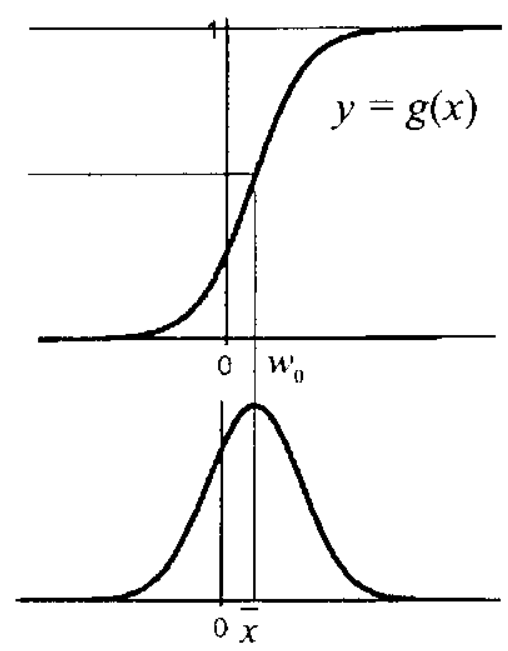

Figura 4.6: Uma entrada $x$ com densidade $p_{x}(x)$, nesse caso uma densidade normal, passa por uma função não linear $g(x)$. Como resultado, a média da entrada, $\bar{x}$, deve coincidir com o termo $w_{0}$ e a variância da entrada. $\sigma^{2}$, deve coincidir com a inclinação da função $g(x)$.

Para a função tangente liperbólica,

$$
\begin{aligned}
& y=\tanh (u), \quad u=w x+w_{0} \\
& y^{\prime}=\frac{\partial y}{\partial u}=1-y^{2}
\end{aligned}
$$

obtém-se as seguintes reglats des aprendizado:

$$
\begin{aligned}
& \Delta w \propto \frac{1}{w}-2 x y \\
& \Delta w_{0} \propto-2 y .
\end{aligned}
$$

As derivaçōes completas para as regras nas Equaçōes (4.34) e (4.35) podem ser encontradas no Apêndice A.

Pode-se observar que as regras para $w$, apresentadas nas Equaçōes (4.30) e (4.34), possuem um termo anti-Hebbiano, $x(1-2 y)$ (função logística) ou $-2 x y$ (função tangente hiperbólica), e um termo anti-decaimento $1 / u$. Esse termo tem o papel de evitar duas situações nas quais a saida $y$ torna-se não informativa: (i) quando os valores de $y$ ficam saturados em 0 ou 1 e (ii) quando $w$ é tão pequeno que o valor de $y$ permanece por volta de 0.5 .

O efcito dessas rogras ó prodizir uma distribuição de saj́da $f_{y}(y)$ que seja próxima à distribuição uniforme. cue é a distribuição com entropia máxima para uma variável limitada 
entre 0 e 1.

A seguir, será mostrado como essa abordagem pode ser adequada a uma rede neural artificial com entradas e saidas multidimensionais.

\subsubsection{Regras de Aprendizado ICA para uma Rede Neural com $N$ Entradas e $N$ Saídas}

Considere mana rede neural com un vetor de entrada $\mathbf{x} \in \mathbb{R}^{N}$, isto é, $\mathbf{x}$ é $N$-dimensional, uma matriz de pesos $\mathbf{W}$. um vetor de ruído $\mathbf{w}_{0}$ e um vetor de saídas $\mathbf{y}=g\left(\mathbf{W} \mathbf{x}+\mathbf{w}_{0}\right) \in \mathbb{R}^{N}$. De maneira análoga à Equação (4.24), é possível relacionar $p_{\mathbf{x}}(\mathbf{x}) \operatorname{com} p_{\mathbf{y}}(\mathbf{y})$ pelo determinante da matriz Jacobiana $\mathbf{J}(\mathbf{x})$, da scguinte forma (Papoulis 1991):

$$
p_{\mathbf{y}}(\mathbf{y})=\frac{p_{\mathbf{x}}(\mathbf{x})}{|\mathbf{J}|}
$$

onde $|\mathbf{J}|$ é o valor absoluto do determinante da matriz Jacobiana.

$\Lambda$ derivação das regras para a adaptação de $\mathbf{W}$ e $\mathbf{w}_{0}$, neste caso, procede como no caso para uma uma entrada e uma saída, visto anteriormente. No entanto, por estar considerando vetores no espaço $\mathbb{R}^{N}$. ao invés de maximizar $\ln |\partial y / \partial x|$, deve-se maximizar $\ln |\mathbf{J}|$. Essa quantidade representa o logaritmo do volume de espaço em y no qual os pontos em $\mathbf{x}$ são mapeados. Maximizando-se cssa quantidlade, tenta-se espalhar de forma uniforme, o conjunto de treinamento de pontos $\mathbf{x}$ em $\mathbf{y}$.

Pode-se considerar. primeiramente, o caso de uma rede neural composta por unidades com função de ativação sigmóide. $\mathbf{y}=g(\mathbf{u}), \mathbf{u}=\mathbf{W} \mathbf{x}+\mathbf{w}_{0}$, com $g$ sendo a função logística $g(\mathbf{u})=\left(1+e^{-\mathbf{u}}\right)^{-1}$. As regras de aprendizado derivadas para esse caso são similares às das Equaçòes $(4.30)$ e (4.31):

$$
\begin{array}{ll}
\Delta \mathbf{W} \propto\left[\mathbf{W}^{T}\right]^{-1}+(\mathbf{1}-2 \mathbf{y}) \mathbf{x}^{T} \\
\Delta \mathbf{w}_{0} \propto \mathbf{1}-2 \mathbf{y} .
\end{array}
$$

No entanto, agora $\mathbf{x}, \mathbf{y}, \mathbf{w}_{0}$ e 1 são vctores $\in \mathbb{R}^{N}$ ( 1 é um vetor no qual todos os elementos são iguais a 1), W é uma matriz e o termo anti-Hebbiano tornou-se urm produto interno entre dois vetores. O termo anti-decaimento foi generalizado para um termo anti-redundância: a inversa da transposta da matriz de pesos. Para um peso individual, $w_{i j}$, essa regra equivale à: 


$$
\Delta u_{i j} \propto \frac{\operatorname{cof} w_{i j}}{\operatorname{det} \mathbf{W}}+x_{j}\left(1-2 y_{i}\right)
$$

onde cof $w_{i j}$ é o cofator de $w_{i j}$.

A derivação completa para a regra em (4.37) pode ser encontrada no Apêndice A. Essa regra tem o mesmo efeito da regra para $u$ apresentada na Equação(4.30). $\Lambda$ exceção aqui é que, ao invés do ponto instável da dinâmica da rede ser $w=0$, qualquer matriz $\mathbf{W}$ cujo determinante seja igual zero irá resultar en uma solução degenerada. Nesse caso, para que a rede encontre uma solução estável, as diferentes unidades $y_{i}$ devem aprender a representar aspectos diferentes das entradas. Portanto, quando os vetores de pesos referentes a duas unidades de saida diferentes tornam-se muito parecidos: o determinante de $\mathbf{W}$ torna-se pequeno e a dinâmica natural do aprendizado faz com que esses dois vetores de pesos divirjam entre si. Na regra apresentada na Equação(4.39), esse efeito í atemuado pelo numerador, cof $u_{i j}$. Quando esse cofator torna-se pequeno, pode haver uma indicação de que existe uma degeneração na matriz de pesos do resto dessa camada (ou scja aqueles pesos que não estão associados com a entrada $x_{j}$ ou com a saída $\left.y_{i}\right)$, mostrando que, nesse caso, qualquer degeneração $\mathrm{cm} \mathbf{W}$ tem pouca relação com o peso específico $u_{i j}$ que está sendo ajustado.

Para neurônios cujas funçôes de transferência sejam a função tangente hiperbólica, as seguintes regras de aprendizado são derivadas:

$$
\begin{aligned}
& \Delta \mathbf{W} \propto\left[\mathbf{W}^{T}\right]^{-1}-2 \mathbf{y} \mathbf{x}^{T} \\
& \Delta \mathbf{w}_{0} \propto-2 \mathbf{y} .
\end{aligned}
$$

Generalizando as regras de aprendizado para W, apresentadas nas Equaçōes (4.37) e (4.40), para qualquer funçaō de transferência ou ativaçào $g(\mathbf{u})$, chega-se à seguinte equaçāo:

$$
\Delta \mathbf{W} \times\left[\mathbf{W}^{T}\right]^{-1}+\left(\frac{\frac{\partial p(\mathbf{u})}{\partial \mathbf{u}}}{p(\mathbf{u})}\right) \mathbf{x}^{T}=\left[\mathbf{W}^{T}\right]^{-1}-\varphi(\mathbf{u}) \mathbf{x}^{T},
$$

que é de fundamental importância na derivação do modelo ICAMM, que será apresentado no Capítulo 6. De fato, essa mesma regra pode scr derivada a partir de outros pontos de vista teóricos, tais como por meio das abordagens MLE (Pearlmutter \& Parra 1996) e maximização da negentropia (Girolami \& Fyfe 1997). Uma revisāo dessas técnicas e as relaçōes cntre estas podem ser encontradias em (Lee, Girolami, Bell \& Sejnowski 1998). 


\subsubsection{Equivalência entre Maximização de Informação e Estimaçāo por Máxima Verossimilhança}

Como dito anteriomente, no modelo ICA, as observações $\mathbf{x}$ são assumidas como sendo geradas a partir de variáveis latentes $\mathbf{s}$ por meio de um mapeamento linear $\mathbf{A}$. No caso da ausência de ruído, pode-se usar um estimador paramétrico da densidade $\hat{p}(\mathbf{x} ; \mathbf{a})$ para encontrar um vetor de parâmetros a que minimize a diferença entre o modelo generativo $\hat{p}(\mathbf{x} ; \mathbf{a})$ e a distribuição observada $p(\mathbf{x})$. Os vetores a são considerados os vetores de bases de $\mathbf{A}$, de modo que $\hat{p}(\mathbf{x} ; \mathbf{a})$ ć uma estimativa da densidade $p(\mathbf{x})$ dos vetores observados. A diferença entre a estimativa da densidacle e a densidade das obervações pode ser medida pela divergência KL:

$$
D(p(\mathbf{x}) \| \hat{p}(\mathbf{x}: \mathbf{a}))=\int p(\mathbf{x}) \log \frac{p(\mathbf{x})}{\hat{p}(\mathbf{x} ; \mathbf{a})} d \mathbf{x}=H(\mathbf{x})-\int p(\mathbf{x}) \log \hat{p}(\mathbf{x} ; \mathbf{a}) d \mathbf{x} .
$$

onde $p(\mathbf{x})$ é a fdp dis observaçoes $\mathbf{x}$ e $\hat{p}(\mathbf{x} ; \mathbf{a})$ é uma estimativa paramétrica da distribuiçào $p(\mathbf{x})$. A divergência $D(p(\mathbf{x}) \| \hat{p}(\mathbf{x}: \mathbf{a}))$ ć zcro somente quando a estimativa $\hat{p}(\mathbf{x} ; \mathbf{a})$ for igual à densidade das observaçócs $p(\mathbf{x})$. Pearlmutter e Parra (Pearlmutter \& Parra 1996) e Cardoso (Cardoso 1998) mostraram que as abordagens infomax e MLE são equivalentes para a ICA, como será descrito brevemente aqui.

A verossimilhıuça de que a amostra $\mathbf{X}=\left\{x_{1}, \ldots, x_{T}\right\}$ seja geradas com uma distribuição particular $\hat{p}(\mathbf{x}: \mathbf{a})$ é:

$$
\hat{p}(\mathbf{X} ; \mathbf{a})=\prod_{i=1}^{T} \hat{p}\left(\mathbf{x}_{i} ; \mathbf{a}\right)
$$

Tomando-se o logaritmo dà Equação(4.44) e dividindo a mesma pelo número de observações, resulta na log-verossimiliıança normalizada, dada pela seguinte equaçāo:

$$
L(\mathbf{a})=\frac{1}{T} \sum_{i=1}^{T} \log \hat{p}\left(\mathbf{x}_{i} ; \mathbf{a}\right)
$$

Uma vez que essa equaçào corresponde à média amostral de $\log \hat{p}(\mathbf{X} ; \mathbf{a})$, esta irá convergir, pela lei dos grandes números (ver: por exemplo, (DeGroot 1987)), para a sua esperança matemática:

$$
L(\mathbf{a})=\int p(\mathbf{x}) \log \hat{p}(\mathbf{x} ; \mathbf{a}) d \mathbf{x}
$$

Fazendo $\hat{p}(\mathbf{x} ; \mathbf{a})=\frac{\bar{j}(\mathbf{x} ; \mathbf{a})}{p(\mathbf{x})} p(\mathbf{x})$, a equação anterior pode ser reescrita da seguinte forma: 


$$
\begin{aligned}
L(\mathbf{a}) & =\int p(\mathbf{x}) \log p(\mathbf{x}) d \mathbf{x}-\int p(\mathbf{x}) \log \frac{p(\mathbf{x})}{\hat{p}(\mathbf{x} ; \mathbf{a})} d \mathbf{x} \\
& =H(\mathbf{x})-D(p(\mathbf{x}), \hat{p}(\mathbf{x} \| \mathbf{a})) .
\end{aligned}
$$

Uma vez que $H(\mathbf{x})$ não depende do $\mathbf{W}$, maximizar a log-verossimilhança corresponde a minimizar a divergência KL cntre a densidade das observações $p(\mathbf{x})$ e a densidade estimada $\hat{p}(\mathbf{x} ; \mathbf{a})$,

$$
\frac{\partial L(\mathbf{a})}{\partial \mathbf{W}}=-\frac{\partial}{\partial \mathbf{W}} D(p(\mathbf{x}) \| \hat{p}(\mathbf{x}, \mathbf{a})) .
$$

Dado que A é uma matriz inversivel e a divergência $\mathrm{KL}$ é invariante sob uma transformação inversível. minimizar a divergência KL apresentada na Equaçāo(4.49) equivale a minimizar a divergência entre a densidade das estimativas de fontes $p(\mathbf{u})$ e a densidade das fontes verdadeiras $p(\mathbf{s})$ :

$$
\frac{\partial L(\mathbf{a})}{\partial \mathbf{W}}=-\frac{\partial}{\partial \mathbf{W}} D(p(\mathbf{s}) \| \hat{p}(\mathbf{u})) .
$$

Dessa forma, as Equaçôes (1.50) e (4.20) são equivalcntes para o método ICA.

\subsection{Maximização da Negentropia}

Uma outra mancira para estimar una transformação ICA é por meio da maximização da negentropia dos vatores de $u$. A definiçāo da negentropia $J$ é dada por:

$$
J(\mathbf{u})=H\left(\mathbf{u}_{\text {gauss }}\right)-H(\mathbf{u})
$$

onde $\mathbf{u}_{\text {gauss }}$ é um vetor aleatório gaussiano que apresenta uma matriz de covariância igual à de $\mathbf{u}$. Nesse caso, a negentropia também pode ser interpretada como uma medida de não gaussianidade (Comon 1994).

O conceito de negentropia também pode ser formulado por meio da divergência KL entre $p(\mathbf{u})$ e a distribuição $p_{\text {gous ss }}(\mathbf{u})$ :

$$
J(\mathbf{u})=D\left(p(\mathbf{u}) \mid p_{\text {gauss }}(\mathbf{u})\right)=\int p(\mathbf{u}) \log \frac{p(\mathbf{u})}{p_{\text {gauss }}(\mathbf{u})} d \mathbf{u}
$$

onde $\mathbf{u}=\mathbf{W} \mathbf{x}$ é o vetor de fontes estimadas.

Em (Lee. Girolami. Bcll \& Scjnowski 1998), a negentropia das estimativas $u_{i}$ foi maximizada utilizando o método do gradiente ascendente, resultando na seguinte regra de aprendizado 
$\operatorname{para} \mathbf{W}$ :

$$
\Delta \mathbf{W} \times\left[\mathbf{W}^{T}\right]^{-1}+\left(\frac{\frac{\partial p(\mathbf{u})}{\partial \mathbf{u}}}{p(\mathbf{u})}\right) \mathbf{x}^{T}=\left[\mathbf{W}^{T}\right]^{-1}-\varphi(\mathbf{u}) \mathbf{x}^{T},
$$

Pode-se notar: portanto. que essa regra de aprendizado é equivalente à regra encontrada pela abordagem infomax (ver Equaçào (4.42)), e consequentemente também é equivalente à regra encontrada pela estimação por máxima verossimilhança, apresentada na Seção 4.8.5.

\subsubsection{O Algoritmo FastICA}

O mćtodo do gradiente ascendente, utilizado para derivar a regra de aprendizado da Equação (4.53). possui algmuas limitações. Por exemplo, algoritmos que apresentam regras derivadas por esse método apresentam convergência lenta e dependem de uma boa escolha para os parâmetros de aprendizagent.

O algoritmo FastJCA. que utiliza um esquema de iteração de ponto fixo (Fletcher 1980), foi desenvolvido por Hyvärincn (Hyvärinen 1999) com o objetivo de tornar o processo de estimação ICA mais rápido e confiável. Para isso, foram utilizadas aproximações da medida de negentropia, desenvolvidas em (Hyvärinen 1998). No caso mais simples, essas aproximaçōes sāo da forma:

$$
J\left(u_{i}\right) \approx\left[E\left\{g\left(u_{i}\right)\right\}-E\{g(z)\}\right.
$$

onde a função $g$, denominada de função de contraste, é praticamente qualquer funçào não quadrática e z é uma variável gaussiana de média zcro e variância unitária. A aproximaçào darla por essa equacion fornece una fumção objetivo para estimar a transformação ICA no algoritmo FastICA.

No Fast ICA. a derivaçào da regra de aprendizado para w (considerando cada componente independente isoladamentc) ut.jiza uma aproximação do método de Newton. Detalhes dessa derivação podem ser encontrados em (Hyvärinen 1999) e (Hyvärinen, Karhunen \& Oja 2001).

Neste caso, a regra de atualização para cada w é dada por:

$$
\Delta \mathbf{w}=E\left\{\mathbf{x} g\left(\mathbf{w}^{T} \mathbf{x}\right)-E\left\{g^{\prime}\left(\mathbf{w}^{T} \mathbf{x}\right)\right\} \mathbf{w}\right\}
$$

onde $g$ e $g^{\prime}$ sào, respectivamente, a função de contraste escolhida e a sua derivada.

Em (Hyvärinen 1999). são consideradas as seguintes escolhas para a função de contraste: 


$$
\begin{array}{cl}
g_{1}(u)=\tanh (u) & g_{1}^{\prime}(u)=1-\tanh ^{2}(u) \\
g_{2}(u)-u \exp \left(-u^{2} / 2\right) & g_{2}^{\prime}(u)=\left(1-u^{2}\right) \exp \left(-u^{2} / 2\right) \\
g_{3}(u)=u^{3} & g_{3}^{\prime}(u)=3 u^{2} .
\end{array}
$$

A utilização dessas funçōes cono funçōes de contraste no Fast.ICA foram analisadas em (Hyvärinen 1999) e os benefícios das mesmas podem ser resumidos nos seguintes ítens:

- $g_{1}$ é uma boa função de contraste de propósito geral.

- Quando as componentes independentes forem altamente super-gaussianas, ou quando a robustez for un fator importante a ser considerado, pode ser melhor utilizar a função $g_{2}$.

- A utilização da funçào $g_{3}$ é justificada para estimar componentes independentes subgaussianas, na ausência de ruídos (outliers).

Todavia, o algoritmo FastICA funciona para praticamente qualquer distribuiçâo das componentes independentes c para cqualçuer escolha da função de contraste. A preocupação com tal escolha somente é fundament al quando houver interesse em otimizar o desempenho do método.

O algoritmo FastICA. cujo código cm MATLAB está disponivel em http://www.cis . hut.fi/projects/ica/fastica, foj utilizado neste presente trabalho para estimar a transformaçào de variáveis originais em variáveis esparsas, necessária para a realização do método Sparse Code Shrinkage, que scrá apresentado no próximo capítulo.

\subsection{Considerações Finais}

Neste capítulo, o método ICA foi descrito em detalhes, juntamente com suas suposiçoes, restrições, ambiguidades c condiçōes necessárias à sua aplicação. Além disso, foram apresentadas várias abordagens derivadas a partir de diferentes teorias para a realização desta técnica.

Foi demonstrado que a abordagem infomax e aquela que utiliza o princípio MLE para a derivação de regras de aprendizado ICA são equivalentes. Como será visto no Capítulo 6, essas duas abordagens são consideradas na derivação do modelo ICAMM, originalmente proposto por (Lce, Lcwicki \& Scjuowski 2000) para realizar a classificação não supervisionada em um conjunto de dados. 
A abordagem ICA đerivada pcla maximização da negentropia também foi apresentada e discutida, juntamente com o algoritıno FastICA, que utiliza esse princípio para calcular as matrizes de transformaçào ICA. Esse algoritmo foi utilizado, neste trabalho, para a implementaçâo do método Sparse Code Shrinkage: que será apresentado no Capítulo 5. Além disso, também foi demonstrado que a ałordigem por maximização da negentropia também é equivalente às abordagens infomax c MLF para estimação ICA. 


\section{Capítulo 5}

\section{Pré-Processamento de Imagens}

\subsection{Considerações Iniciais}

Cma imagem pode cstar sujeita a ruídos e interferencias advindos de várias fontes, como por exemplo, sensores clétricos, lentes fotográficas e erros em canais de transmissäo. Para reduzir os efeitos desses ruidus. várias técnicas lineares e não lineares foran propostas e están disponíveis ha literatura (ver, por (xemp)lo, (Gonzalcz \& Woods 1992) e (Pratt 2001)).

Dentre as tícnicas lineares para reduça de ruídos, destaca-sc, por exemplo, a técnica de filtragem no domínio de Fourier (Nathan 1970). Com o objetivo de preservar alguns detalhes de imagens perdidos pela suavizaçào cxcessiva realizada pelas técnicas lineares, foram propostos, mais recentemente, métodos de redução de ruídos que utilizam operadores não lineares. Dentre as técnicas uão lineares mais utilizarlas, destacam-se a técnica de filtragem por mediana (Nodes \& Gallagher 1982) e o método para redução de ruídos baseado em wavelets (Walker 1999).

Lna ontra técnica nă linear para redução de rúdos desenvolvida recentemente é o método Sparse Codk Shrinkuge. o qual tem sido utilizado com sucesso, estabelecendo-se como uma técnica promissora para esse tipo de aplicaçäo (Lu \& Liao 2000) (Szatmáry, Szirtes \& Lörincz 2003). A principal vantagem desse método em relação a outras técnicas não lineares té que este está relacionado an método de estinaçäo ICA, adeçuado para detectar estruturas importantes em diztos de: imagens (Bell \& Sejnowski 1997).

Além de ser aplicado para redução de rúdos, observon-ses, no presente trabalho, que o mótodos SCS. assim como ontras tócnicas desenvolvidas para essa finalidade, também pode funcionar como un filtro de suavizagio para o borranento e remoģăo de pequenos detalhes de uma inagem antes da extraça de chjetos maiores. Com isso, regioes de interesse a serem destacadas podem se tornar mais hommgentexs, facilitando o processo de segmentação. No entanto, um efeito 
indesejado causado por esse tipo de tócnica é que, eventualmentr. características importantes da imagem, como as bordas dos objetos, tambén podem scr borradas, prejudicando o processo de segmentação.

Neste capítulo, é proposta uma metodologia para pré-processamento de imagens, objetivando amenizar o problema da presença de ruídos e suavizar a imagem, sem comprometer, no entanto, características importantes, como os contoruos dos objetos a serem destacados no processo de segmentação.

Mais precisamente, a metodologia de pré-processamento proposta neste trabalho envolve duas principais etapas. No primeiro estágio, o método SCS é aplicado para reduzir o ruído na imagem original, funcionando também como um filtro do suavizacãu para o borramento de pequenos detalhes na imagem. Logo após, uma imagem resultante da aplicaça do operador de Sobel (Gonzalez \& Woods 1992) à imagem original é combinada com a imagem processada pelo método SCS. A seguir, esses dois métodos serão descritos, assim como um maior detalhamento das etapas envolvidas na aplicação da metodologia.

\subsection{Método Sparse Code Shrinkage para Redução de Ruídos cm Imagens}

Sparse Coding (Barlow 1994), (Ficld 1994), (Olshansen \& Field 1996) é um método originalmente desenvolvido para encontrar uma representacia de rede neural para dados multidimensionais na qual somente poucos neurônios săo significativamente ativades ao mesmo tompo. De forma equivalente, isso significa que um determinado neuronio raramente é ativado. Denota-se por $\mathrm{x}=\left(x_{1}, x_{2}, \ldots, x_{n}\right)^{T}$ o vetor alcatório observado n-dinemsional. que 6 dado como entrada para una rede neural e por $\mathbf{s}=\left(s_{1}, s_{2}, \ldots, s_{n}\right)^{T}$ o vetor de variáveis transformadas, que são as $n$ saídas lincares da rede. Denotando-se ainda os vetores de pesos dos neurönios por $\mathbf{w}_{i}, i=1, \ldots, n$ e por $\mathbf{W}=\left(\mathbf{w}_{1}, \mathbf{w}_{2}, \ldots, \mathbf{w}_{n}\right)^{T}$ a matriz de pesos cujas colunas são os vetores de pesos, a relaçào linear é dada por:

$$
\mathrm{s}=\mathbf{W} \mathbf{x}
$$

F́ assumido, nesse modelo, que o número de componentes esparsas isto ć, o número de:

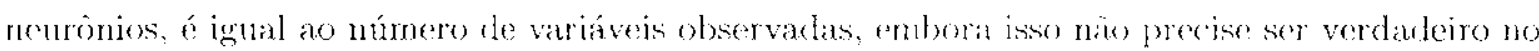
caso geral (Olshausen \& Ficld 1997). O método Sparse Coding pode, agora. scr formulado como urn método de busca por uma matriz de pesos $\mathbf{W}$, tal que as ompronentes $\mathrm{s}_{i}$ sejan täe esparsas 
quanto possivel. Unua variável aleatória $\mathbf{s}_{i}$ com média zero é considerada esparsa quando esta possuir uma fungăo densidade de probabilidade com um pico cm zero e caudas pesadas, como é o caso, por exemplo. da distribriçăo de Laplace (ver Figura X). Em aspectos práticos, esparsidade é equivalente à supergauscianidade (Hyvärinen \& Ojd 1997) ou leptokurtosis (kurtosis positiva) (Kendall \& Stuart 1958).

O método Sparse Coding está intrinsicamente relacionado ao método ICA (Hyvärinen. Hoyer \& Oja 2001), cule 6 aj)resentado, em detallhes, no Capítulo 4. No modelo de dados utilizado no método ICA, é postulado que $\mathrm{x}$ é uma transformaçào linear de componentes independentes: $\mathbf{x}=$ As.

Invertendo-se essa relaçio, obtém-se a Fquação (5.1), com $\mathbf{W}=\mathbf{A}^{1}{ }^{1}$. Além disso, foi provado que a estimaçio do mordelo de dados ICA pode ser reduzida à busca por direcóes não correlacionlatas nas quais as componentes săo tăo nāo gausssianas quanto possível (Comon 1994), (Hyvärinen 1999). So as componentes independentes forem esparsas (mais precisamente, supergaussianas), o método reduz-se à busca por projeçós não correlacionadas que possuam

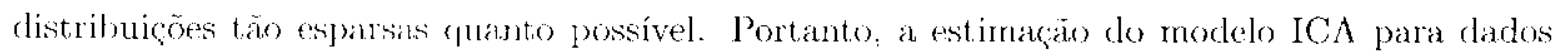
esparsos é equivaleute ao método Sparse Coding sob a restrição de que as componentes sejam não correlacionadas. Essa conexäo com o método ICA tambén mostra claramentc que o método Sparse Coding pode ser considerado como um método para reduçāo de redundância, o que, de fato, foi um dos primciros olijetivos do Sparse Coding (Barlow 1994), (Field 1994).

Em (Hyvärinen. Hoycr \& Oja 2001), o método Sparse Code Shrinkage (SCS) para supressia de ruídos é apresentado e analisado. De acordo com essa abordagem, dado um sinal corrompido por ruido aditivo gaussiano, aplica-se o método para reduçáo do rúclo por meio de uma liniarizaño (shrinkagr) slave das componentes esparsas. Intuitivamente, una ver que somente poucos neurônins estarão ativos (isto é, com ativação significativamente diferente de zero) simmltaneamente ern un código esparso, pode-se assumir que as atividades dos neurónios

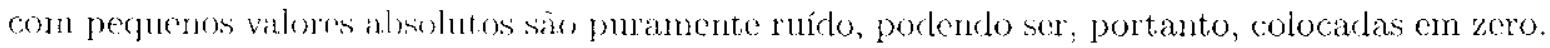
Dessa forma, retém-se somuente as poucas componentes com ativaçoes significantes. O método SCS também pode ser relacionado com técnicas baseadas em wavelels (Donoho, Jonhstonc, Kerkyacharian \& Picard 1995). (Simoncelli \& Adelson 1996). De fato, o sparse coding pode ser visto como um método alaptativo para determinar bases ortogonais semelhantes a wavelets, baseando-se somente nos dados (observaçoes) disponíveis. 


\subsubsection{Redução de Ruídos em Variáveis Aleatórias Não-Gaussianas}

O ponto de partida para a derivação do método Sparse Code Shrinkage é o fato de que ass distribuiçóns das componentes esparsas são não-gaussianas. Portanto, é possível desenvolver uma teoria geral que mostra como remover ruídos gaussianos do variáveis escalares nào-gaussianas. O método Sparse Code Shrinkage é baseado na Estimaçăo por Máxima Vorossilbança (MLE) de variáveis nâo-gaussianas que estão corrompidas por ruido gaussiano.

No caso unidimensional, denota-se por $s$ a variável aleatória não-gaussiana a ser estimada e por $v$ o ruído gaussiano de média zero e variancia $\sigma^{2}$. Assumindo-se também que somente a variável y é obscrvada:

$$
y=s+v
$$

deseja-se estimar a variável original s. Denota-se, agora, for $p$ a densilluke de probabilidade do $s$ e por $f=-\log p$ o negativo do logaritmo da densidade $p$. Em (Iyvärinen. Hoyer \& Oja 2001), utilizou-se o método MLE, juntamente com algurnas aproximaçós para concontrar o seguinte estimador ${ }^{1}$ para $s$ :

$$
\hat{s}=g(y)=\operatorname{sinal}(y) \max \left(0,|y|-\sigma^{2}\left|f^{\prime}(y)\right|\right) .
$$

\subsubsection{Modelagem para Densidades Esparsas}

Uma densidade esparsa (super-gaussiana) clássica é a distribuiçâo de Laplane (ver Figma 5.1), também conhecida como distribuição dupla-exponencial. A modelagem do método SCS para a distribuição de Laplace é considerada importante pois esta ó frequentemente cucontrada em dados de imagens (Otshausen \& Field 1996).

A função de densidade de probabilidade (fdp) para uma distribuição de Laplace é darda por:

$$
p(s)=\frac{1}{\sqrt{2}} \exp (-\sqrt{2}|s|)
$$

Para essa densidade, a não linearidade $g$, apresentada na Equagaio (5.3) assume a forma:

$$
\hat{s}-g(y)=\sin a \mid(y) \max \left(0,|y|-\sqrt{2} \sigma^{2}\right) .
$$

Essa funçào é considerada uma função de limiarização (shrinkage) uma vez que esta é utilizada

\footnotetext{
${ }^{3}$ Fisse estimador também poele ser chamado ele Estimador Miximu a Penterieri.
} 


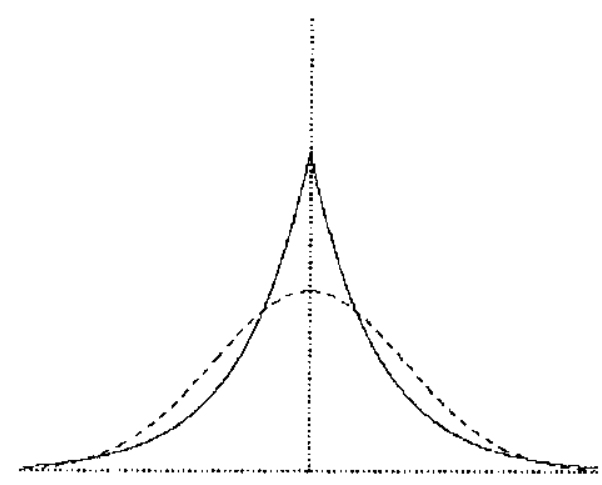

Figura 5.1: Função de densidade da distribuiçăo de Laplace, que ć uma distribuição supergaussiana clássica. Pura efuito do comparação, a densidade gaussiana (normal) é dada pela curva pontilhada. A densidade laplaciana tem um pico mais agudo em zero e caudas mais pesadas do que a distribugio gamsiana.

para reduzir o valor absoluto do seu argumento, com base no nível (cstimado pela variância $\sigma^{2}$ ) do ruído gaussiano. Dessa forma, argumentos com valores pequenos são colocados em zero.

Intuitivannente, a utilislade da função apresentada na Equação (5.5) pode ser explicada como segue. Uma vez que a densidade de uma variável aleatória super-gaussiana (por exemplo, uma variável com distribuicăo de Laplace) apresenta-se na forma de uma curva com um pico acentuado en zero (ver Figna 5.1), pode-se assumir que pequenos valores de $y$ correspondem somente a ruídos, isto r. a s 0 . A limiarizacão desses valores para zero deve, portanto, causar a redução dos ruílos e uma sulavizaçăo na imagem original.

\subsubsection{Transformação dos Dados em Variáveis Esparsas}

Na secäo auterior, foi apresentado como reduzir ruídos gaussianos cm variáveis aleatórias não ganssianas unidimensionais. por meio da abordagem MLf. Para a redução de ruídos en urn vetor aleatório. a operagào de shrinkage pode ser aplicada separadamente a cata una das componentes do vetor en questán.

Porem antes de aplicar o operador de shrinkage, é desejável transformar linearmente os dados originais em variáveis esparsas para que o método para redução de ruídos seja o mais eficiente possível. Para isso, em (Hyvärinen, Ifoyer \& Oja 2001), é considerada a estimaşão do modelo generativo de dados ICA , dado por:

$$
x-\text { As. }
$$


assumindo-se que as variáveis latentes $s_{i}$ são independentes e näo-gaussianas e A é uma matriz quadrada de coeficientes escalares.

O modelo ICA, apresentado em detalhes no Capítulo 4. tem-se mostrado capaz de descrever alguns aspectos importantes de estruturas estatist icas básicus de alta ordem presentes em dados de imagens (Olshausen \& Field 1996) (Olshausen \& Field 1997) (Bell \& Sejnowski 1997) Dessa forma, modelando os dados de imagens com o modelo ICA a possivel obter um método simples para reduzir o ruído no vetor $\mathrm{x}$ da seguinte forma: primciro encontra-se as estimativas $\hat{s}_{i}$ das componentes independentes, c depois. reconstrói-se o vetor $\mathrm{x}$ como: $\hat{\mathrm{x}}=\hat{\mathrm{A}} \hat{\mathrm{s}}$.

A cstimativa $\hat{s}$ pode ser obtida simplesmente aplicando-so a não lincaridado de shrinkage apresentarla na Equação (5.5) aos componentes de $\mathbf{s}=\mathbf{W} \mathbf{x}$, onde $\mathbf{W}=\hat{\mathbf{A}}^{-1}$ porde ser cncontrado utilizando-se qualquer método ICA convencional (ver Capítulo 4).

A scguir ó apresentado um resumo do algoritmo SCS, descrito nitu seçoes anteriores. Nesse método, um operador para reduçäo de rúdos é aplicado nas componentes esparsas dos dados. Dessa forma, antes de proceder com essa operação. uma transformaçio bascada $\mathrm{m}$ ICA deve ser aplicada aos dados originais para maximizar a esparsidade das componentes. Fssa restriçào a variáveis esparsas é justificada pelo fato de que em muitas aplicaçoos, por exemplo cm tarefas de processamento de imagens, as distribuiçoes encontradias sän esparsas.

O algoritmo SCS segue, então, os seguintes passos (Ityvïrinen, Oja \& I Inyer 1998):

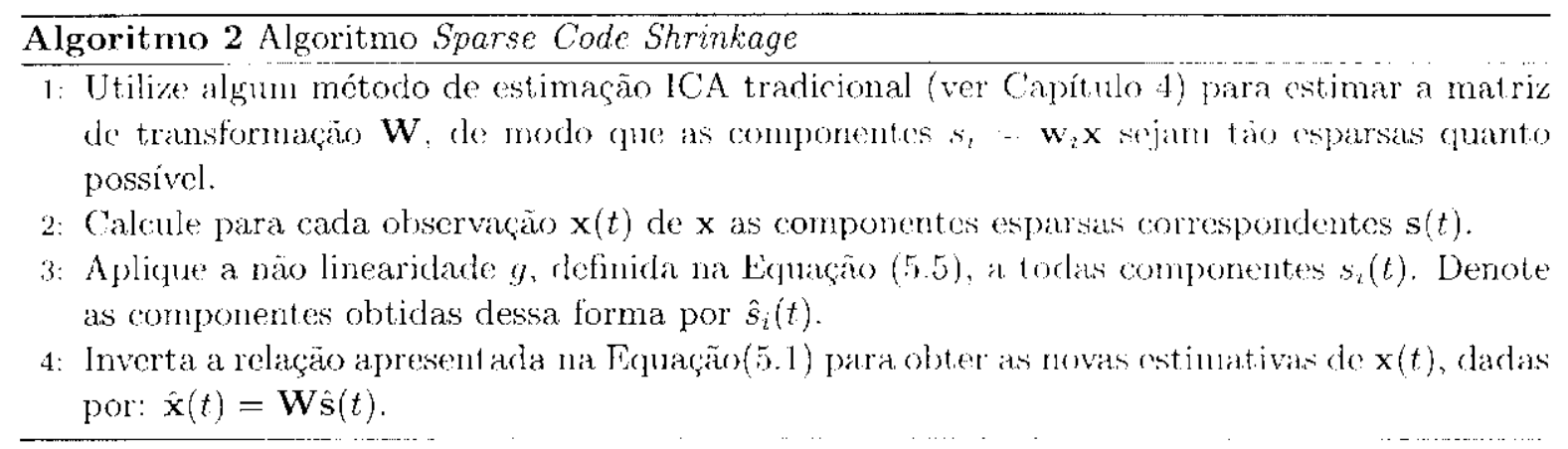

Maiores detalhes da derivação do método SCS podem ser encontrates fm (Hyvärinen, Hoyer \& Oja 2001), onde também são apresentados resultados experinentais com dados de imagens e conexóes do SCS com outros mótodos não lineares para restuçiono de rúdos.

O método SCS foi aplicado nos cxperimentos do redução de rúdos em imagens reali-

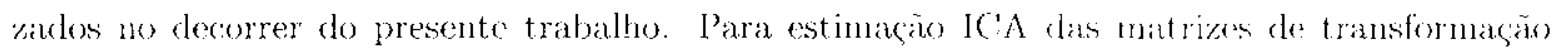
W foi utilizado o algoritmo FastICA (Hyvärinen 1999), que utiliza o princípio da negentropia, apresentiado no Capitulo 4. 


\subsection{Detector de Bordas de Sobel}

A detergäo de bordas é a ahordagem mais comum para a deteçảo de descontinuidades significantes nos niveis de cinza de mma innagem (Gonzalez \& Woods 1992). A formulaçăo do algoritmo de deicciño de bordas do Sobel, assume que as regiões de uma imagem são suficientemente homogêneas, de maneira que a transiçäo entre duas regiões (denominada de borda) podo ser determinada somente pela descontinuidade dos níveis de cinza. Para detectar essas descontinuidades, o detector de Sobel biseia-se no cálculo de um operador local diferencial, dado pelo vetor griadiente.

Para uma funçio $f(x, y)$, o volor gradiente de $f$ nas coordenadas $(x, y)$ de uma imagem é definido da seguinte forma:

$$
\nabla \mathbf{f}-\left[\begin{array}{ll}
C_{x}^{x} & C_{y}
\end{array}\right]=\left[\begin{array}{ll}
\frac{\partial f}{\partial x} & \frac{\partial f}{\partial y}
\end{array}\right]
$$

A idéia básica por trás do método de Sobel é que a norma do vetor gradiente, geralmente chamada simplesmente de gradiente, dada por:

$$
\|\nabla f\|-\left[\left.\frac{\partial f^{2}}{\partial x} \frac{\partial f^{2}}{\partial y}\right|^{1 / 2}\right.
$$

pode ser usada na deteccüo de bordas de uma imagem.

Frequentemente, pode-se aproximar a norma do vetor gradicnte pelos valores absolulos das derivadas parciais:

$$
\nabla f=\left|G_{x}\right|+\left|G_{i}\right|
$$

para simplificar a implenentaçác do método.

Na prática, a obtençäo das derivadas parciais $C_{x}$ e $C_{y}$ na posição de cada pixel é implementada por meio da utilizaçăo de duas máscaras, denominadas de operadores de Sobel, que podem ser vistass na Figura 5.2 .

Considera-se, entrão, a regiāo de uma imagem mostrada na Figura 5.2(a), onde os valores de $z$ representam os níveis de cinza de cada ponto da imagen.

$\Lambda$ Equaço (5.9) pode ser aincla aproximada no ponto $z_{5}$ utilizando-se os seguintes cálculos para $C_{x} \in G_{y}$ : 


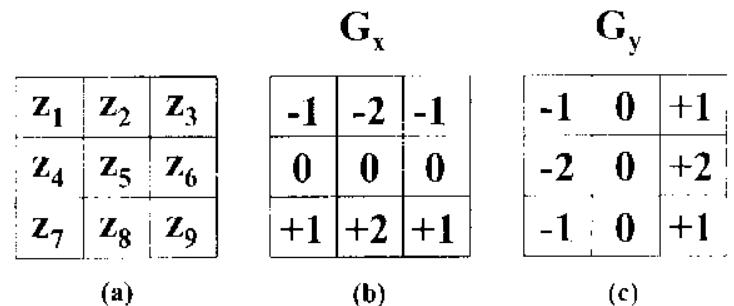

(a)

(b)

(c)

Fignra 5.2: Máscaras de Sobel. (a) Região de uma imagem de tamanho $3 \times 3$. (b) Máscara. usada no cálculo de de $G_{x}$ no ponto central da região. (c) Máscara usada no cálculo de de $G_{y}$ no ponto central da região.

$$
\begin{aligned}
& G_{x}(t)=\left(z_{7}+2 z_{8}+z_{9}\right)-\left(z_{1}+2 z_{2}+z_{3}\right) \\
& G_{y}(t)-\left(z_{3}+2 z_{6}+z_{9}\right)-\left(z_{1}+2 z_{1}+z_{7}\right)
\end{aligned}
$$

Dessa forma, a aplicaçño das máscaras apresentadas nas Figunas 5.2(b) e 5.2(c) a tocłas as posições (pixels) da imagem, resulta em uma irragen de gradiente do mesmo tamanho da intagem original.

\subsection{Metodologia Proposta}

Nesta seção são apresentados alguns detalhes de implementaçüo on exemplo da aplicaçâo sa metodologia de pré-processamento proposta no presente trahalha. En linhas gerais, o método SCS e o detector de bordas de Sobel säo combinados para coustruir uma imagem, a partir da imagem original, que apresente caracteristicas mais adecluadas para um processo de segmentaçá. Primeiramente, o método SCS é aplicado com as finalidades de reducão de ruídos e suavização da imagem original, tornando as áreas de maior interesie mais lomogeneas. Paralelamente, o detector de bordas de Sobel é aplicado à imagcm original, com a tinalidade de ressaltar bordas importantes. O resultado dessas duas operações são, ontão, combinalos. para gerar a imagem pré-processada. Uma ilustraçào desse esquema é apresentada na k'igura 5.3.

Na implementação do método SCS, foi utilizado, para estimação ICA das matrizes de transformagào W, o algoritmo lastiCA (Iyvärinem 1999). que e babeado no princípro da estimação por negentropia, apresentado no Capítulo 4. () código em MATLAB do FastICA, pronto para sele aplicado a dados de imagens, esté disponivel no enderceo http://www. cis. hut.fi/ projects/ica/imageica/. Dessa forma, uma vez encontrada a matriz de transformação esparsa W para uma imagem original, esta é tranformata em shas componontes esparsas, sobre 


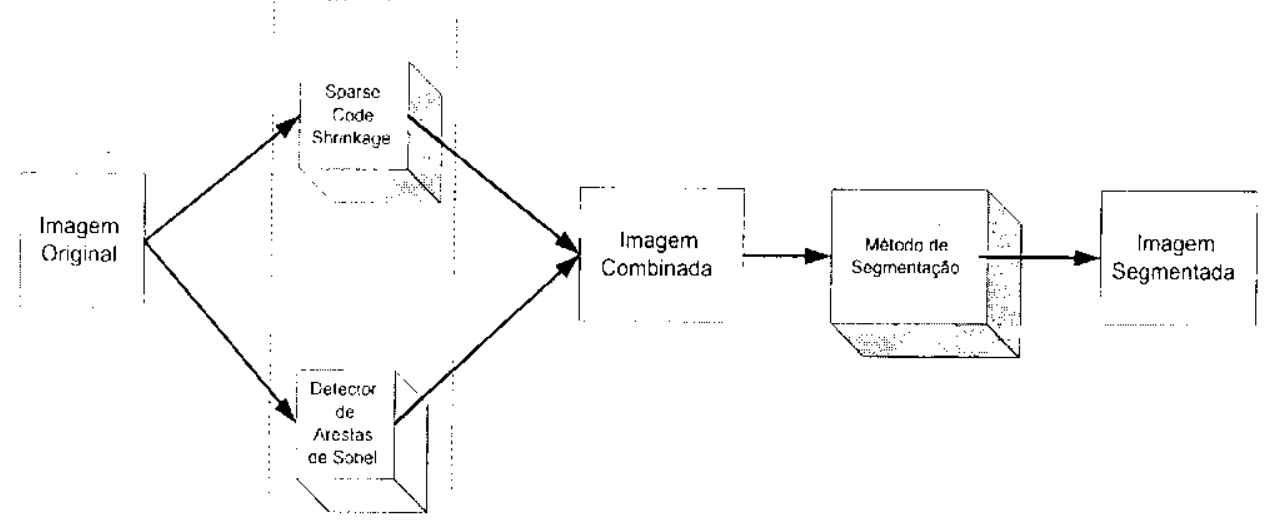

Figura 5.3: Metodologia de pré-processamento proposta. No primeiro estágio, são aplicados à imagem original, cm paralelo, o método Sparse Code Shrinkage (SCS) e detector de bordas de Sobel. Os resultados desse estágio são combinados, gerando com a imagem pré-processada. Finalmente, um método de segmentacia pode ser aplicado à inagem resultante desse processo.

ats quais é aplicauda a funçäo de shrinkage apresentada na Equação (5.5).

Para a aplicação do detector de bordas de Sobel foi utilizada a função edge, pertencente ao pacote (toolbox) de processamento de imagens do MATLAB. Essa função considera uma imagem cm niveis de cinza $I$ como entrada e devolve uma imagem binária do mesmo tamanho de $I$, em que l's são colocados nas posiçoes em que a funçäo detecta urna borda e 0's nas posições onde bordas não foram identificaklas. Além do método de Sobel, a função edge pode implementar outros operadores para deteção de bordas, como os de Prewit.t, Roberts e Canny.

\subsubsection{Exemplo de uma Aplicação da Metodologia Proposta}

Para ilustrar o funcionamento da metodologia proposta, pode-se tomar como exemplo o pré-processamento d i imagem l.ena, de domínio público, apresentada na Figura 5.4(a), na sua versão original. As imagens resultantes da aplicaçáo do SCS e do detector de Sobel são apresentadas nas Figuras 5.4 (b) c $5.4(\mathrm{c})$, respectivamente. Fssas duas imagens foram então combinadas (sobrepostasi) para gerar a imagem na Figura 5.4(d), que representa o resultado final obtido pelia aplicaçáo da metodologia propostat.

\subsection{Considerações Finais}

Neste capitules, foi apresentadia a metodologia para préprocessamento de imagens, alue consiste em combinat a aplicação do método SCS e do detector de bordas de Sobel a imagem 


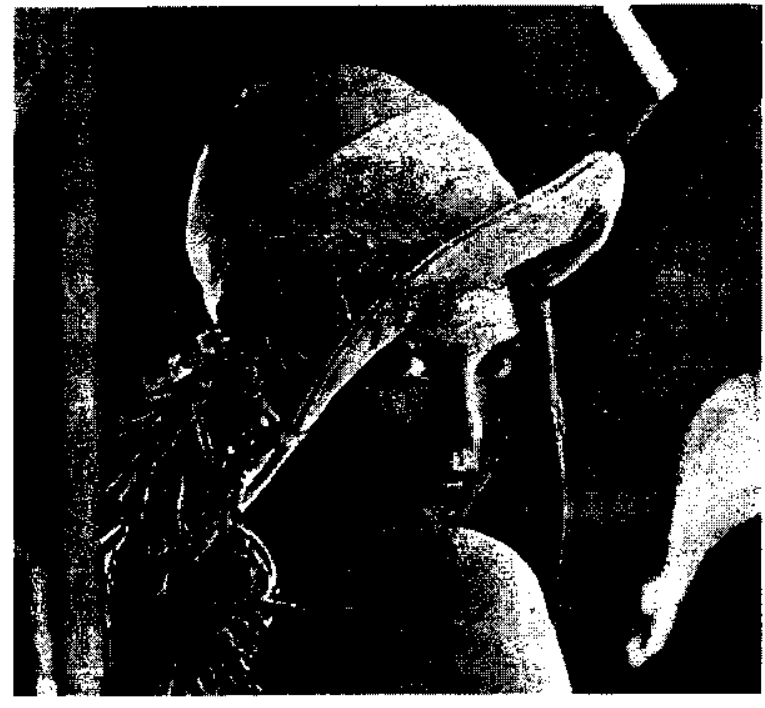

(a)

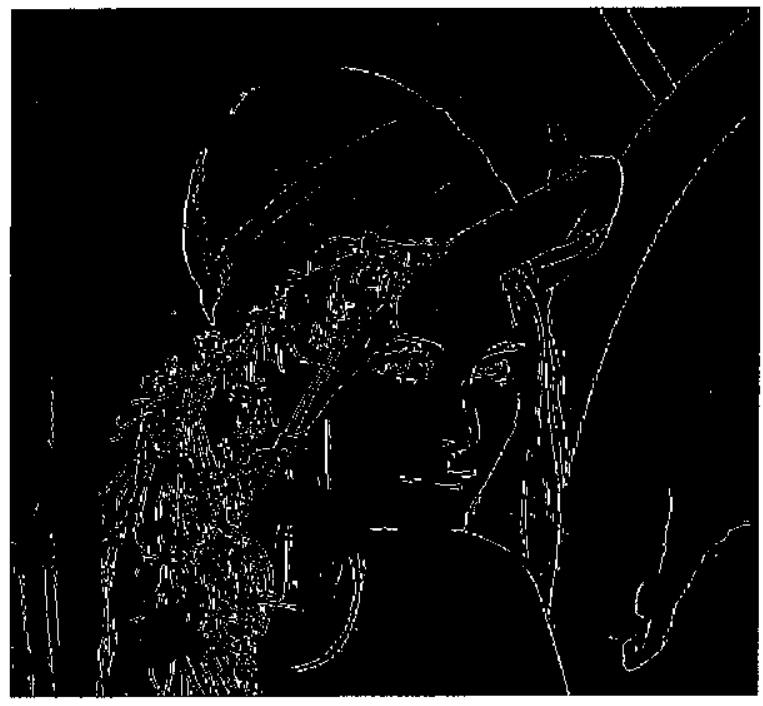

(i)

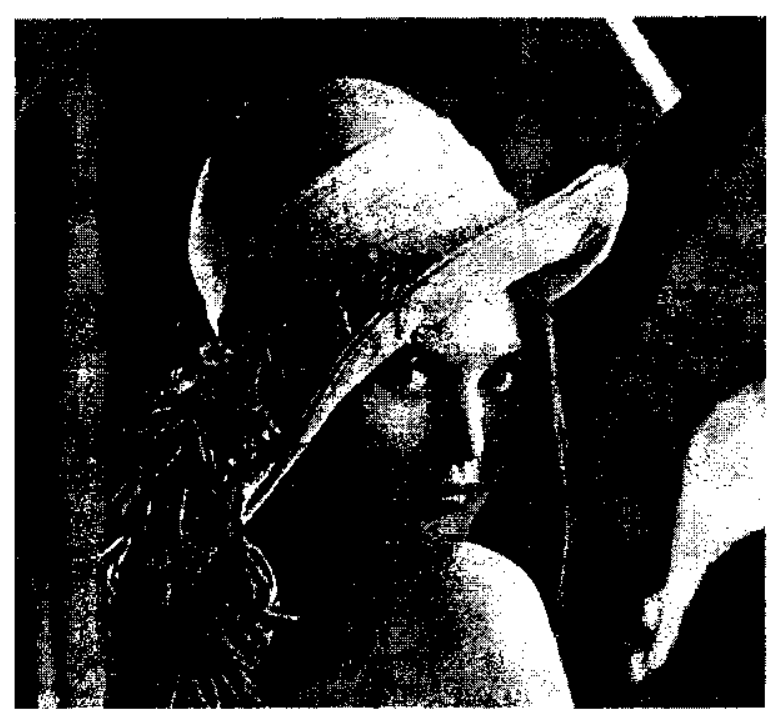

(1)

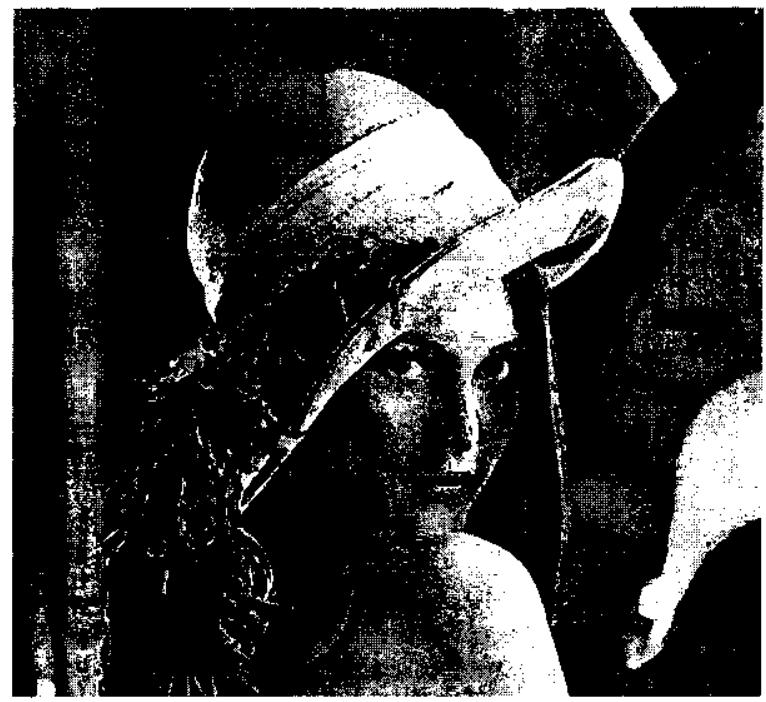

(d)

Figura 5.4: Fxemplo da aplicação da metodologia de pré-processimento. (a) hnagem original. (b) Imagem resultante da aplicaçăo do SCS. (c) Imagem resultante da aplicasäa do detector de bordas de Sobel. (d) Resultado final obtido pela aplicacias) (la metcrdologia proposta.

origintal.

O método SCS, que foi originalmente proposto para redução de rúrfos, aqui é aplicado também cono filtro de suavizaça de imagens, de modo a tormar mais homogêneas algumas 
regiōes de interesse. facilitando, portanto. o processo de segmentação. Já a funcionalidade do detector de bordas de Sobel é realçar os contornos de objetos borrados pela suavização da imagem, uma ve\% que essas características também são importantes para tarefas de segmentação.

Os doin mútodos guc compöm a metodologia proposta foram apresentados neste capítulo, juntamente com um exemplo da aplicação dessa metodologia, para ilustrar o funcionamento da mesmiat.

No Capitulo \&. seräu apresentados experimentos que mostram como a utilizaçăo dessa metodologia acarreton en mollorias nos resultados de segmentaçào de imagens obtidos pelos métodos auto-organizáveis considerados neste trabalho. 


\section{Capítulo 6}

\section{Modelo de Misturas ICA}

\subsection{Considerações Iniciais}

Classificação e agrupamento de padrões são problemas encontrados com frequência em vários campos da ciência, cono biologia, medicina, visão computacional e inteligência artjficial (Jain, Duin \& Mao 2000). Um Jroblema de classificação de padrões pode ser resolvido computacionalmente por algoritmos supervisionados on não supervisionados (Watanabe 1985). Em uma classificação supervisionatla. cacla padrão do conjunto de dados é identificado como sendo um monbro de uma classes predefinida. Já um algoritmo de classificação nāo supcrvisionado associa cada padrão a uma classe baseando-se somente em estatísticas. sem nenhum conhecimento sobre as classes de treinamento.

Uma abordagen para classificação não supervisionada é baseada em modelos de misturas (ver, por exemplo. (Duda \& Hart 1973: Bishop 1994)), nos quais a distribuição dos dados é modelada como uma soma ponderada de densidades condicionais. Por exemplo, no caso de um modelo de misturas (iaussianas (também chamadas de normais), assume-se que os dados em cada classe têm uma distribuição Gaussiana multivariada. Entretanto, essa suposição implica que o modelo de misturas Gaussianas explora somente estatísticas de segunda ordem (médias e covariâncias) dos dados observados para estimar as densidades a posteriori.

O Modelo de Misturas ICA (ICAMM) ${ }^{1}$ foi proposto por Lee e colaboradores em (Lee, Lewicki \& Sejnowski 2000). com o objetivo de superar uma linitaçāo da técnica ICA, que consiste na suposição de que as fontes geradoras dos dados são independentes. Em tal abordagem, essa suposição foi relaxada utilizando-se o conceito de modelo de misturas.

Cada classe no ICAMM é descrita por uma combinação linear de fontes independentes com

'do original, em ingles, JCA Mixture Model. 
densidades não Gaussianas. O algoritmo encontra as componentes independentes e a matriz de bases para carla classe utilizando o algoritmo de aprendizagem infomax estendido (Lee, Girolami \& Sejnowski 1999) e também calcula a probabilidade de pertinência de classe para cada padrão do conjunto de dados. As regras de aprendizado para o ICAMM foram derivadas utilizando o método de otimização do gradiente ascendente para maximizar a função de log-verossimilhança dos dados.

\subsection{Formulação do Modelo ICAMM}

No decorrer deste capitulo. assume-se que os dados $N$-dimensionais $\mathbf{X}=\mathbf{x}_{1}, \ldots, \mathbf{x}_{T}$ são gerados independentemente por um modelo de misturas de densidades (Duda \& Hart 1973). A verossimilhança dos dados é dada pela densidade conjunta:

$$
p(\mathbf{X} \mid \Theta)=\prod_{t=1}^{T} p\left(\mathbf{x}_{t} \mid \Theta\right)
$$

sendo a log-verossimilhança. portanto, dada pela seguinte equação:

$$
L=\sum_{t=1}^{T} \log p\left(\mathbf{x}_{t} \mid \boldsymbol{\Theta}\right) .
$$

A densidade das misturas é dará por

$$
p\left(\mathbf{x}_{\ell} \mid \Theta\right)=\sum_{t=1}^{K} p\left(\mathbf{x}_{t} \mid C_{k}, \theta_{k}\right) p\left(C_{k}\right),
$$

onde $\Theta=\left(\theta_{1} \ldots \theta_{k}\right)$ hüo os parâmetros desconhecidos para cada $p\left(\mathbf{x} \mid C_{k}, \theta_{k}\right)$, chamadas de densidades das componentes da mistura. Nesse caso, $C_{k}$ denota a classe $k$ e assume-se que o número de classes, $K$, é conhecido de antemão. Assume-se, ainda que as densidades das componentes são não gaussianas e que os dados em cada classe são descritos por:

$$
\mathbf{x}_{i}=\mathbf{A}_{k} \mathbf{s}_{k}+\mathbf{b}_{k},
$$

onde $\mathbf{A}_{k}$ é uma matriz cscalar $N \times N^{2}$ e $\mathbf{b}_{k}$ é o vetor de ruído para a classe $k$. O vetor $\mathbf{s}_{k}$ é chamado de vetor de fontes para classe $k$.

$\Lambda$ meta do ICAMM é classificar um conjunto de dados não rotulados e determinar os parâmetros para cada classe $\left(\mathbf{A}_{k}, \mathbf{b}_{k}\right)$ e as probabilidades condicionais $p\left(C_{k} \mid \mathbf{x}_{t}, \boldsymbol{\Theta}\right)$ para todos

\footnotetext{
${ }^{2}$ Neste caso, o nimaro de fontes cigual ao número de sensores.
} 
os dados do conjunto.

O algoritmo iterativo çue realiza a subida do gradiente da funçāo de log-verossimilhança dos dados (ver Equlação 6.2) possui os seguintes passos:

- Compute a log-verossimilhança dos dados para cada classe:

$$
\log p\left(\mathbf{x}_{t} \mid C_{k}, \theta_{k}\right)=\log p\left(\mathbf{s}_{k}\right)-\log \left(\left|\operatorname{det}\left(\mathbf{A}_{k}\right)\right|\right)
$$

onde $\theta_{k}=\left\{\mathbf{A}_{k}, \mathbf{b}_{k}\right\}$. Nesse caso, $\mathbf{s}_{k}=\mathbf{A}_{k}^{-1}\left(\mathbf{x}_{t}-\mathbf{b}_{k}\right)$ é implicitamente modelado para a adaptação de $\mathbf{A}_{k}$ :

- Compute a probabilidado para cada classe, dado o vetor de dados $\mathbf{x}_{t}$ :

$$
p\left(C_{k} \mid \mathbf{x}_{t}, \boldsymbol{\Theta}\right)=\frac{p\left(\mathbf{x}_{t} \mid C_{k}, \theta_{k}\right) p\left(C_{k}\right)}{\sum_{k} p\left(\mathbf{x}_{t} \mid C_{k} \theta_{k}\right) p\left(C_{k}\right)}
$$

- Adapte as matrizes $\mathbf{A}_{k}$ e os vetores de bias $\mathbf{b}_{k}$ para cada classe, utilizando as seguintes regras:

$$
\Delta \mathbf{A}_{k} \times-p\left(C_{k} \mid \mathbf{x}_{t}, \boldsymbol{\Theta}\right) \mathbf{A}_{k}\left[\mathbf{I}-\mathbf{K} \tanh \left(\mathbf{s}_{k}\right) \mathbf{s}_{k}^{T}-\mathbf{s}_{k} \mathbf{s}_{k}^{T}\right]
$$

e

$$
\mathbf{b}_{k}=\frac{\sum_{t} \mathbf{x}_{t} p\left(C_{k} \mid \mathbf{x}_{t}, \Theta\right)}{\sum_{t} p\left(C_{k} \mid \mathbf{x}_{t}, \Theta\right)}
$$

em que $t=1, \ldots, T$ e $\mathbf{K}$ f́ uma matriz diagonal $N$-dimensional cujos elementos $k_{k, 2}$ são adaptados da seguinte forma:

$$
k_{k, 2}=\operatorname{sign}\left(E\left\{\operatorname{sech}^{2}\left(s_{k, i, t}\right)\right\} E\left\{s_{k, i, t}^{2}\right\}-E\left\{\left[\tanh \left(s_{k, i, t}\right)\right] s_{k, i, t}\right\}\right)
$$

A distribuição é considlerada supergaussiana quando $k_{k, i}=1$ e subgaussiana quando $k_{k, i}=$ -1 (Girolami 1998).

Como discutido no Capítulo 4, existem vários métodos para adaptar as matrizes $\mathbf{A}_{k}$ no modelo ICA. O algoritmo ICAMM é bascado na regra de aprendizado ICA infomax estendida (Lee, Girolami \& Sejnowski 1999), que possibilita separar fontes desconhecidas com distribuiçōes sub e super-gaussianas. Essia scparação é atingida por meio de um tipo simples de regra de aprendizado que foi primeiramente derivada por Girolami (Girolami 1998). A regra de aprendizado 
em (Lee, Girolami \& Sejnowski 1999) utiliza a análise de estabilidade de (Cardoso 1998) para alternar entre regimes sub c super-gaussianos.

Para a estimação da função log-verossimilhança da Equação (6.5), o termo $\log p\left(\mathbf{s}_{k}\right)$ é modelado como segue:

$$
\log p\left(\mathbf{s}_{k, t}\right) \propto-\sum_{i=1}^{N}\left(k_{k, i} \log \left(\cosh s_{k, i, t}\right)-\frac{s_{k, i, t}^{2}}{2}\right)
$$

\subsection{Derivação do Algoritmo ICAMM}

Para a derivaçăo do algoritmo ICAMM, assume-sc que $p(\mathbf{X} \mid \Theta)$, como dado na Equação (6.1) é uma funçào diferenciável de $\Theta$. Lembrando que a log-verossimilhança $L$ dos dados $\dot{c}$ dada por:

$$
L=\sum_{t=1}^{T} \log p\left(\mathbf{x}_{\ell} \mid \boldsymbol{\Theta}\right)
$$

e, usando a Equação (6.3). o gradiente para os parâmetros de cada classe $k$ é:

$$
\begin{aligned}
\nabla_{0_{k}} L & =\sum_{t=1}^{T} \frac{1}{p\left(\mathbf{x}_{t} \mid \Theta\right)} \nabla_{\theta_{k}} p\left(\mathbf{x}_{t} \mid \Theta\right) \\
& =\sum_{i=1}^{T} \frac{\nabla_{\theta_{k}}\left[\sum_{k=1}^{K} p\left(\mathbf{x}_{t} \mid C_{k}, \theta_{k}\right) p\left(C_{k}\right)\right]}{p\left(\mathbf{x}_{t} \mid \Theta\right)} \\
& =\sum_{t=1}^{T} \frac{\nabla_{\theta_{k}} p\left(\mathbf{x}_{t} \mid C_{k}, \theta_{k}\right) p\left(C_{k}\right)}{p\left(\mathbf{x}_{t} \mid \Theta\right)}
\end{aligned}
$$

Usando a regra de Bayes (ver. por exemplo (Papoulis 1991)) a probabilidade de classe para urn vetor de dados $\mathbf{x}_{t}$ ć:

$$
p\left(C_{k} \mid \mathbf{x}_{t}, \Theta\right)=\frac{p\left(\mathbf{x}_{t} \mid \theta_{k}, C_{k}\right) p\left(C_{k}\right)}{\sum_{k} p\left(\mathbf{x}_{t} \mid \theta_{k}, C_{k}\right) p\left(C_{k}\right)}
$$

Substituindo a Equação (6.13) na Equação (6.12), tem-se: 


$$
\begin{aligned}
\nabla_{\theta_{k}} L & =\sum_{t=1}^{T} p\left(C_{k} \mid \mathbf{x}_{t}, \Theta\right) \frac{\nabla_{\theta_{k}} p\left(\mathbf{x}_{t} \mid \theta_{k}, C_{k}\right) p\left(C_{k}\right)}{p\left(\mathbf{x}_{t} \mid \theta_{k}, C_{k}\right) p\left(C_{k}\right)} \\
& =\sum_{t=1}^{T} p\left(C_{k} \mid \mathbf{x}_{t}, \Theta\right) \nabla_{\theta_{k}} \log p\left(\mathbf{x}_{t} \mid C_{k}, \theta_{k}\right)
\end{aligned}
$$

A funçào de log-verossinithança na Equaçào (6.14) representa a log-verossimilhança para cada classe. No modelo ICAMM, a log-verossimilhança de classe ć dada pela log-verossimilhança para o modelo ICA padrão:

$$
\begin{aligned}
\log p\left(\mathbf{x}_{1} \mid C_{k}, \theta_{k}\right) & =\log \frac{p\left(\mathbf{s}_{k}\right)}{\left|\operatorname{det} \mathbf{A}_{k}\right|} \\
& =\log p\left(\mathbf{A}_{k}^{-1}\left(\mathbf{x}_{t}-\mathbf{b}_{k}\right)\right)-\log \left|\operatorname{det} \mathbf{A}_{k}\right|
\end{aligned}
$$

Em (Lee. lfwicki \&: Scjnowsiki 2000), o método do gradiente ascendente ć utilizado para estimar os parâmetros que maximizam a função de log-verossimilhança. Os parâmetros do gradiente para cada classe săo o gradiente da matriz de bases $\mathbf{A}_{k}$ e o gradiente do vetor de ruído $\mathbf{b}_{k}, \nabla_{\theta_{k}} L=\left\{\nabla_{\mathbf{A}_{k}} L, \nabla_{\mathrm{b}_{k}} L\right\}$, os quais serão considerados, um por vez, a seguir.

\subsubsection{Estimação das Matrizes de Bases}

As matrizes de bases $\mathbf{A}_{k}$ para cada classe podem ser atualizadas utilizando a Equação (6.14).

$$
\nabla_{\mathbf{A}_{k}} L=\sum_{t=1}^{T} p\left(C_{k} \mid \mathbf{x}_{i}, \boldsymbol{\Theta}\right) \nabla_{\mathbf{A}_{k}} \log p\left(\mathbf{x}_{t} \mid C_{k}, \theta_{k}\right) .
$$

A atualizaçào (adiataçăo) é realizada utilizando o método do gradiente ascendente do gradiente dia densidade de componentes cm relaçāo às matrizes de base, resultando em:

$$
\Delta \mathbf{A}_{k} \propto p\left(C_{k} \mid \mathbf{x}_{i}, \boldsymbol{\Theta}\right) \nabla_{\mathbf{A}_{k}} \log p\left(\mathbf{x}_{\ell} \mid C_{k}, \theta_{k}\right)
$$

Pode-se notar que na adlaptação das matrizes de bases, o gradiente da densidade de componentes com respeito às matrizes de bases $\mathbf{A}_{k}$ é ponderado por $p\left(C_{k} \mid \mathbf{x}_{i}, \Theta\right)$.

A computação de $\nabla_{\mathbf{A}_{k}} \log p\left(\mathbf{x}_{1} \mid C_{k}, \theta_{k}\right)$ pode ser realizada por meio da técnica de ICA. A regra de aprendizagem para as matrizes de bases $\mathbf{A}_{k}$ pode ser derivada a partir de vários pontos de vista teóricos, tais como por meiu das abordagens MLE (Pearlmutter \& Parra 1996), infomax 
(Bell \& Sejnowski 1995) e maximizaçào da negentropia (Girolami \& Fyfe 1997).

O algoritmo de aprendizado para o ICAMM é derivado em (Lee, Lewicki \& Sejnowski 2000) utilizando a Estimação por Máxima Verossimilhança (MLE) ${ }^{3}$. A abordagem MLE para separação cegia de fontes foi primeiramente proposta por Gacta e Lacoume (Gaeta \& Lacoume 1990) e Pham e Garrat (Pham \& Garrat 1997), sendo mais recentemente pesquisada por Peralmutter and Parra (Pcarmutter \& Parra 1996) e Cardoso (Cardoso 1997). A função densidade de probabilidade das observaçōes $\mathbf{x}$ pode ser expressa como (Papoulis 1991) (Amari \& Cardoso 1997):

$$
p(\mathbf{x})=|\operatorname{det} \mathbf{W}| p(\mathbf{u})
$$

em que $p(\mathbf{u})=\prod_{i=1}^{N} p_{i}\left(u_{2}\right)$ é a supost a distribuição de $p(\mathbf{s})$. A log-verossimilhança da Equação (6.18) ć:

$$
L(\mathbf{u} . \mathbf{W})=\log |\operatorname{det} \mathbf{W}|+\sum_{i=1}^{N} \log p_{i}\left(u_{i}\right) .
$$

Como det $\mathbf{W}=\sum_{j} w_{i j} \operatorname{cof}\left(w_{i j}\right)$ para algum $j$, onde $\operatorname{cof}^{T}(\mathbf{W})$ denota a transposta de $\operatorname{cof}(\mathbf{W})$ e $\mathbf{W}^{-1}=\frac{1}{\operatorname{det} \mathbf{W}} \operatorname{cof}^{T}(\mathbf{W})$, tems-se que, para o primeiro termo da Equação $(6.19)$ :

$$
\frac{\partial}{\partial \mathbf{W}} \log |\operatorname{det} \mathbf{W}|=\frac{\mathbf{J}}{\operatorname{det} \mathbf{W}} \operatorname{cof}(\mathbf{W})=\left(\mathbf{W}^{T}\right)^{-1}
$$

Por sua vez, o segundo termo da Equação (6.19) é derivado da seguinte forma:

$$
\begin{aligned}
\frac{\partial}{\partial \mathbf{W}} \sum_{i=1}^{N} \log p_{i}\left(u_{i}\right) & =\frac{\partial}{\partial \mathbf{W}} \log p_{1}\left(u_{2}\right)+\ldots+\frac{\partial}{\partial \mathbf{W}} \log p_{N}\left(u_{N}\right)= \\
& =\left[\frac{\frac{\partial p_{1}\left(u_{1}\right)}{\partial u_{1}} \frac{\partial u_{1}}{\partial \mathbf{W}}}{p_{1}\left(u_{1}\right)}+\ldots+\frac{\frac{\partial p_{N}\left(u_{N}\right)}{\partial u_{N}} \frac{\partial u_{N}}{\partial \mathbf{W}}}{p_{N}\left(u_{N}\right)}\right]= \\
& =\left[\frac{\frac{\partial p_{1}\left(u_{2}\right)}{\partial u_{1}}}{p_{1}\left(u_{1}\right)}, \ldots, \frac{\frac{\partial p_{N}\left(u_{N}\right)}{\partial u_{N}}}{p_{N}\left(u_{N}\right)}\right]^{T}\left[x_{1} x_{2} \ldots x_{N}\right] .
\end{aligned}
$$

Denominando:

$$
\varphi(\mathbf{u})=-\frac{\frac{\partial p(\mathbf{u})}{\partial u}}{p(\mathbf{u})}=\left[-\frac{\frac{\partial p\left(u_{1}\right)}{\partial u_{1}}}{p\left(u_{i}\right)}, \ldots,-\frac{\frac{\partial p\left(u_{N}\right)}{\partial u_{N}}}{p\left(u_{N}\right)}\right]^{T}
$$

\footnotetext{
${ }^{3}$ do original, $\mathrm{cm}$ ingles, Maximum-Likelihood Estimation.
} 
maximizar a log-verossimilhança com respeito à $\mathbf{W}$, resulta na seguinte regra de aprendizado para $\mathbf{W}$ :

$$
\Delta \mathbf{W} \propto\left[\left(\mathbf{W}^{T}\right)^{-1}-\varphi(\mathbf{u}) \mathbf{x}^{T}\right],
$$

que é, por sua vez, idênticà à Equação(4.42), derivada por meio da abordagem infomax (Bell \& Scjnowski 1995).

Uma mancira ainda mais eficiente para maximizar a log-verossimilhança é seguir o gradiente "natural" (Amari, Cichocki \& Yang 1996), obtido multiplicando-se o gradiente calculado no ponto $\mathbf{W}$, pela direita, pela matriz $\mathbf{W}^{T} \mathbf{W}$. O gradiente natural é utilizado neste caso pois simplifica a regra de aprendizado da Equação (6.23) e acelera a convergência do modelo (Amari 1998). A aplicação do termo de gradiente natural resulta, portanto, na seguinte regra de gradiente descendente para funçào de log-verossimilhança dos dados:

$$
\Delta \mathbf{W} \times \frac{\partial L(\mathbf{u}, \mathbf{W})}{\partial \mathbf{W}} \mathbf{W}^{T} \mathbf{W}
$$

Utilizando o fato de que $\mathbf{x}^{T}=\mathbf{u}^{T}\left(\mathbf{W}^{T}\right)^{-1}$ a Equação (6.23) pode ser reescrita da seguinte forma:

$$
\Delta \mathbf{W} \times\left[\left(\mathbf{W}^{T}\right)^{1}-\varphi(\mathbf{u}) \mathbf{u}^{T}\left(\mathbf{W}^{T}\right)^{-1}\right]
$$

Colocando o termo $\left(\mathbf{W}^{T}\right)^{-1}$ en cvidéncia, tem-se que:

$$
\Delta \mathbf{W} \times\left[\mathbf{I}-\varphi(\mathbf{u}) \mathbf{u}^{T}\right]\left(\mathbf{W}^{T}\right)^{-1}
$$

Por fim, multiplicando a Equisine (6.26). pela direita, pelo termo $\mathbf{W}^{T} \mathbf{W}$ do gradiente natural, obtém-se:

$$
\begin{aligned}
& \Delta \mathbf{W} \times\left[\mathbf{I}-\varphi(\mathbf{u}) \mathbf{u}^{T}\right]\left(\mathbf{W}^{T}\right)^{-1} \mathbf{W}^{T} \mathbf{W}= \\
& \Delta \mathbf{W} \times\left[\mathbf{I}-\varphi(\mathbf{u}) \mathbf{u}^{T}\right] \mathbf{W} .
\end{aligned}
$$

A estimativa de densidade paramétrica $p_{i}\left(u_{i}\right)$ desempenha um papel essencial para o sucesso da regra de aprendizadio da Equação (6.28). A convergência local é assegurada se $p_{i}\left(u_{i}\right)$ for uma estimativa da verdadeira densidade das fontes (Pham \& Garrat 1997). Por exemplo, a funçāo sigmóide usada no algoritmo de Bell c Sejnowski (Bell \& Sejnowski 1995) ć adequada para separar fontes supergaussianas, isto é, fdp's com caudas mais pesadas do que a distribuição 
gaussiana.

Uma naneira de generalizar a regra de aprendizado para fontes com distribuiçōes subgaussianas ou supergaussianas é derivar regras de aprendizado separadas para componentes subgaussianas e supergaussianas. Para isso é necessário adequar, para cada caso, o termo não linear $\varphi(u)$, que é dependente da fdp das estimativas de fontes. No modelo infomax (Bell \&. Sejnowski 1995), apresentado na Seção (4.8.2), os termos nāo lineares $\varphi(u)$ foram derivados somente para distribuiçòes de fontes supergaussianas. Dessa forma, a abordagem infomax original apresenta a limitação de não separar fontes que possuem densidades subgaussianas. $O$ algortimo proposto em (Girolami 1998) a aprekenta derivaçōes para termos nāo lineares $\varphi(u)$ que permitcm realizar a separaçâo estável de fontes supergaussianas e subgaussianas.

Una densidade simétrica estritamente subgaussiana pode ser modelada utilizando-sc uma forma simétrica do modelo de misturas de Pearson (Pcarson 1894), como segue (Girolami 1998):

$$
p(u)=\frac{1}{2}\left(N\left(\mu, \sigma^{2}\right)+N\left(-\mu, \sigma^{2}\right)\right)
$$

em que $N\left(\mu, \sigma^{2}\right)$ é a densidade normal com média $\mu$ e variância $\sigma^{2}$. Fazendo $\mu=1$ e $\sigma^{2}=1$, a Equação (6.22) fica reduzida a:

$$
\varphi(u)=-\frac{\frac{\partial p(u)}{\partial u}}{p(u)}=u-\tanh (u)
$$

Para fontes com densidades supergaussianas, pode-se adotar a seguinte densidade:

$$
p(u) \propto N(u) \operatorname{sech}^{2}(u),
$$

em que $N(u)$ é una densidarde normal com média zero com variância unitária. A não linearidade $\varphi(u)$ é, agora:

$$
\varphi(u)=-\frac{\frac{\partial p(u)}{\partial u}}{p(u)}=u+\tanh (u) .
$$

As Equações (6.29) e (6.31) podem ser combinadas como:

$$
\begin{gathered}
\Delta \mathbf{W} \times\left[\mathbf{I}-\mathbf{K} \tanh (\mathbf{u}) \mathbf{u}^{T}-\mathbf{u u}^{T}\right] \mathbf{W} \\
\begin{cases}k_{i}=1 & \text { supergaussiana } \\
k_{i}=-1 & \text { subgaussiana, }\end{cases}
\end{gathered}
$$


em que $k_{i}$ são os elementess da matriz diagonal $N$-dimensional $\mathbf{K}$. Os elementos $k_{i}$ 's foram derivados a partir de uma análise formulada em (Cardoso 1998). A utilização dessa abordagem resultou na seguinte escolha para feita os $k_{i}$ 's, feita em (Lee, Girolami \& Sejnowski 1999):

$$
k_{i}=\operatorname{sign}\left(E\left\{\operatorname{sech}^{2}\left(s_{i, t}\right)\right\} E\left\{s_{i, t}^{2}\right\}-E\left\{\left[\tanh \left(s_{i, t}\right)\right] s_{i, t}\right\}\right) .
$$

o que assegura a estabilidade da regra de aprendizado.

\subsubsection{Regras de Aprendizado do Modelo ICAMM}

Pode-se escrever an Equarsess (6.32) e (6.36) ell termos das matrizes de bases $\mathbf{A}_{k}$ para cada classe:

$$
\Delta \mathbf{A}_{k:} \propto-p\left(C_{k} \mid \mathbf{x}_{t}, \Theta\right) \mathbf{A}_{k}\left[\mathbf{I}-\mathbf{K} \tanh \left(\mathbf{s}_{k}\right) \mathbf{s}_{k}^{T}-\mathbf{s}_{k} \mathbf{s}_{k}^{T}\right]
$$

em que:

$$
\mathbf{s}_{k}=\mathbf{A}_{k}^{-1}\left(\mathbf{x}_{t}-\mathbf{b}_{k}\right)
$$

$\Theta$

$$
k_{i}=\operatorname{sign}\left(E\left\{\operatorname{sech}^{2}\left(s_{i, t}\right)\right\} E\left\{s_{i, t}^{2}\right\}-E\left\{\left[\tanh \left(s_{i, t}\right)\right] s_{i, t}\right\}\right) .
$$

A distribuiçăo da fonte é supergaussiana quando $k_{k, i}=1$ e subgaussiana quando $k_{k, i}=-1$. A adaptação do logaritmo da probabilidade a priori $\log p\left(\mathbf{s}_{k}\right)$ pode ser aproximada da seguinte forma:

$$
\log p\left(s_{k}\right) x-\sum_{i=1}^{N}\left(k_{k, i} \log \left(\cosh s_{k, i, t}\right)-\frac{s_{k, i, t}^{2}}{2}\right) .
$$

\subsubsection{Estimação dos Vetores de Bias}

A Equação (6.14) pode ser utilizada para adaptar os vetores de bias $\mathbf{b}_{k}$ para cada classe, da seguinte forma:

$$
\nabla_{\mathrm{b}_{k}} L=\sum_{t=1}^{T} p\left(C_{k} \mid \mathbf{x}_{t}, \Theta\right) \nabla_{\mathbf{b}_{k}} \log p\left(\mathbf{x}_{t} \mid C_{k}, \theta_{k}\right) .
$$

A adaptação é realizada utilizando-se a subida do gradiente da densidade das componentes com respeito ao vetor de bias $\mathbf{b}_{k}$, resultando em: 


$$
\Delta \mathbf{b}_{k}=\propto p\left(C_{k} \mid \mathbf{x}_{t}, \boldsymbol{\Theta}\right) \nabla_{\mathbf{b}_{k}} \log p\left(\mathbf{x}_{t} \mid C_{k}, \theta_{k}\right)
$$

Utilizando a Equação (6.15) na Equação (6.39), é possível adaptar $\mathbf{b}_{k}$ como segue:

$$
\Delta \mathbf{b}_{k} \propto p\left(C_{k} \mid \mathbf{x}_{t}, \Theta\right) \nabla_{\mathbf{b}_{k}}\left[\log p\left(\mathbf{A}_{k}^{-1}\left(\mathbf{x}_{\ell}-\mathbf{b}_{k}\right)\right)-\log \left|\operatorname{det} \mathbf{A}_{k}\right|\right]
$$

Entretanto. ao invés do utilizar a informação de gradiente, cm (Lee, Lewicki \& Sejnowski 2000), foi utilizada una fórmula aproximada para a adaptaçāo do vetor de bias. Nessa aproximação, foi assumido que:

$$
\begin{aligned}
\nabla_{\mathrm{b}_{k}} L & =0 \\
\sum_{t=1}^{T} p\left(C_{k} \mid \mathbf{x}_{t}, \Theta\right) \nabla_{\mathbf{b}_{k}} \log p\left(\mathbf{x}_{t} \mid C_{k}, \theta_{k}\right) & =0
\end{aligned}
$$

A substituição dia Equasăo (6.15) na Equação (6.41) mostra que o gradiente do primeiro termo da Equação (6.15) tem que ser igual a zcro. A partir dessa conclusäo, segue que:

$$
\nabla_{\mathbf{b}_{k}} \log p\left(\mathbf{A}_{k}^{-1}\left(\mathbf{x}_{t}-\mathbf{b}_{k}\right)\right)=0
$$

Na derivação aprescntada cm (Lee, Lewicki \& Scjnowski 2000), assume-se ainda se que

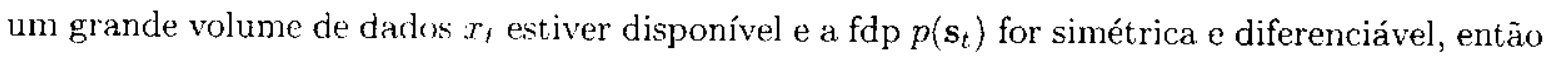
$\log p\left(\mathbf{s}_{t}\right)$ também será simétrico c o vetor de bias pode ser, portanto, aproximado pela média ponderada das observaçoes:

$$
\mathbf{b}_{k}=\frac{\sum_{t} \mathbf{x}_{t} p\left(C_{k} \mid \mathbf{x}_{t}, \boldsymbol{\Theta}\right)}{\sum_{t} p\left(C_{k} \mid \mathbf{x}_{t}, \boldsymbol{\Theta}\right)}
$$

\subsection{Problemas do ICAMM}

Apesar de algumas caracteristicas promissoras do ICAMM terem sido reportadas em (Lec, Lewicki \& Scjnowski 2000), no presente trabalho, apesar das inúmeras tentativas, não foi obtido sucesso na reprodução dos resultados experimentais obtidos por Lee e colaboradores. Ao invés do bom descmpenho de classificação do método, relatado no trabalho original, foram observados em experimentos com dados simulados e com o conjunto de dados de flores Iris, uma convergência muito lenta e resultados de classificação insatisfatórios. Em dois artigos encontrados na literatura (Ridder, Kittler \& Duin 2000), (Shah, Arora, Robila \& Varshney 
2002), o ICAMM também foi aplicado sem resultados expressivos. Em (Ridder, Kittler \& Duin 2000), o ICAMM teve um bon descmpenho para dados 2-D simulados, porém a vantagem de utilizar o método para segmentar imagens nào foi provada conclusivamente. Em (Shah, Arora, Robila \& Varshney 2002), algumas técnicas de extração de características foram consideradas como etapas de pré-processamento para reduzir a dimensionalidade dos dados, na tentativa de aumentar a eficiencia do ICAMN1. Apesar das precisōes mćdias de classificação obtidas por essa abordagem terem side maiores do que aquelas obtidas pelo método $k$-médias (MacQueen 1967). os autores cncontraran algumas limitações e suposiçōes que comprometem o uso do ICAMM cm classificaçào de dados de sensoreamento remoto.

Un dos problemas associados ao ICAMM está relacionado ao fato que o scu algoritmo de aprendizagem é baseado cm una técnica de otimização de gradiente. Portanto, foi observado que o desempenho do mótodo ć afotado, entre outros fatores, por algumas linitaçoes conhecidas associadas a esse tipo de abordagem. Além, disso, na tentativa de entender melhor o seu funcionamento, foram detcctados, neste trabalho, alguns aspectos relativos a sua formulaşão e implementação que poderiann ser melhorados para tornar o método mais eficiente. Tais propostas de melhorias deram origem ao modelo EICAMM, que será apresentado no próximo capitulo.

\subsection{Considerações Finais}

Nestc capítulo, foi apresentado, em detalhes, o Modelo ICAMM, inicialmente proposto para realizar classificação não supervisionada em um conjunto de dados. Porém algumas limitações que coniprometom a utilizaçào ICAMM foram obscrvadas no decorrer do presente trabalho, como será demonstrato no Capítulo 8.

No próximo capítulo. apresentado o Modelo EICAMM, proposto neste trabaho com o objetivo de melhorar o desempenho do ICAMM, por meio da incorporação de técnicas de otimização näo lincares e modificaçoes envolvendo aspectos de modelagem e implementação. 


\section{Capítulo 7}

\section{Modelo de Misturas ICA Aperfeiçoado (EICAMM)}

\subsection{Considerações Iniciais}

- Corr o objetivo de melhorar o desempenho do ICAMM, este capítulo apresenta o Modelo do Misturas ICA Aprrfciçuado (EICAMM), que implementa algumas modificações no ICAMM originalmente proposto. Assin como no ICAMM original, algumas modificações também são baseadas na abordagem de maximização de informação para separação cega de fontes e deconvoluçäo cega de fontes. proposta por Bell e Sejnowski em (Bell \& Sejnowski 1995).

$\mathrm{O}$ desenvolvinento do modelo EICAMM baseia-se, de fato, em algumas melhorias que foram adicionadas ao ICANM original por meio da incorporaçāo de algumas características de métodus de otimização nào lincar. Nesse sentido, o método de Levenberg-Marquardi (ver, por exemplo, (Masters 1995)) foi incorporado ao algoritmo de aprendizagem para garantir e melhorar a convergência do modelo. Além disso, foram também considerados aspectos de modelagem e implementaçāo. Essass modificaçós, que deram origem ao modelo auto-organizável proposto neste tralualho. são apresentadas a seguir.

\subsection{Reformulação do Modelo de Classes}

Ao invés de considerar o termo de bias adicionado aos dados depois que estes foram gerados por um modelo ICA (ver Equação (6.4)), no EICAMM, os vetores de bias são adicionados aos sinais das fontes geradoras. A partir dessa modificação, origina-se uma outra equaçāo, diferente daquela correspondente no ICAMM, para descrever os dados em cada classe, dada por: 


$$
\mathbf{x}_{i}=\mathbf{A}_{k}\left(\mathbf{s}_{k}+\mathbf{b}_{k}\right)
$$

A justificativa para essa modificação reside no fato que, na abordagem infomax, supōc-se a ausencia de rúdos nos sensores $\mathbf{x}$. ou no máximo a existência de sinais com poucos ruídos aditivos. Portanto com o objetivo de garantir a ausência de ruídos adicionados ans sensores. cstes rúdos são consirlerados como adicionados às fontes $\mathbf{s}$, antes do processo de mistura do acordo com o modtelo gemerativo ICA apresentado na Equação (4.3).

\subsection{Regra de Aprendizado para os Termos de Bias}

Com o objetivo de formular uma regra de aprendizado mais informativa para os termos de bias $\mathbf{b}_{k}$, no EICAMM. una nova regra de aprendizado é derivada utilizando a abordagem proposta em (Boll \& Scjnowsi 1995) para maximizar a informação mútua que a saída $Y$ de um processador de rede neural possui sobre a sua entrada $X$. De acordo com essa idéia, quando uma única entrada $x$ passia através de una funçào de transferência não linear $g(x)$, resulta numa variável de saída $y$. de modo que a informação mútua entre essas duas variáveis é maximizada. Por meio dessa transfomaçio, as partes de alta densidade da fdp de $x$ alinha-se com as partes de alta inclinação da função $g(x)$. como apresentado na Figura (4.5).

Dessa forma. as regras de aprendizado para pesos e biases em uma rede neural, de acordo com a abordagem em (Bell \& Sejnowski 1995). são formuladas utilizando alguma função de transfercncia não lincar. Na formulagão do EICAMM, uma nova regra de aprendizado para os termos de bias $\mathbf{b}_{k}$. para cada classe é proposta, considerando-se, aqui, a funçāo de transferência como scndo a tangente hiperbólica:

$$
\Delta \mathbf{b}_{k}=-2 \tanh \left(\mathbf{s}_{k}\right)
$$

As vantagens dessa nowa regra para atualização dos vetores de bias em relaçāo à regra correspondente no ICAMM são:

- A regra do EICAMM ć adaptativa, ao contrário da regra correspondente no ICAMM, no sentido que esta leva em conta, na iteração corrente, os resultados obtidos nas iterações anteriores. Essa vantagem pode ser verificada comparando-se as Equaçōes (6.43) e (7.2) para atualização dos termos de bics nos modelos ICAMM e EICAMM, respectivamente.

- Essa nova regra é formulada de acordo com a teoria de maximização de informaçāo formalmente proposta em (Bell \& Sejnowski 1995), o que leva ao relaxamento da suposição na 
que se bascia a regra para a adaptação dos termos de bias do ICAMM, de que um grande volume de dados de entrada esteja disponível.

\subsection{Ortogonalização das Matrizes de Bases}

Na derivação do modcłu ICAMM, considera-se que $\mathbf{W}_{k}=\mathbf{A}^{-1}$. Dessa forma, a Equação(6.32), derivada em (Lec. Lewicki \& Scjnowski 2000) para a adaptação das matrizes de bases pode ser reescrita como:

$$
\Delta \mathbf{W} \times\left[\mathbf{1}-\mathbf{K} \tanh (\mathbf{u}) \mathbf{u}^{T}-\mathbf{u} \mathbf{u}^{T}\right] \mathbf{A}^{-1}
$$

Entretanto, para que a passagem na derivação feita em (Lee, Lewicki \& Sejnowski 2000) da Equação(6.32) para a Equasauo(6.34) possa ser realizada, deve-se considerar que as matrizes de bases estimadas pelo modelo sejam ortogonais.

Dessa forma. una outra modificação no ICAMM foi incorporada ao modelo EICAMM por meio da suposição de quc as matrizes $\mathbf{A}_{k}$ são ortogonais, de modo que $\mathbf{A}^{-1}=A^{T}$.

Portanto, a regra do adaptaçăo para as matrizes de bases $\mathbf{A}_{k}$ no ElCAMM é dada por:

$$
\Delta \mathbf{A}_{k} \times p\left(C_{k} \mid \mathbf{x}_{t}, \theta\right) \mathbf{A}_{k}\left(\mathbf{I}-\mathbf{K} \tanh \left(\mathbf{s}_{k}\right) \mathbf{s}_{k}^{T}-\mathbf{s}_{k} \mathbf{s}_{k}^{T}\right)^{T},
$$

onde $\mathbf{s}_{k}=\mathbf{A}_{k}^{T} \mathbf{x}_{t}-\mathbf{b}_{k}$. considerando o modelo para os dados de classe utilizado no EICAMM e formalizado na Equação(7.1). Aqui, nota-se que o operador de transposição é utilizado, ao invćs da matriz inversa $\mathbf{A}_{k}^{-1}$ para a modelagem implícita dos vetores de fontes $\mathbf{s}_{k}$, o que implica em uma vantagem computacional do EICANM, cm rclaçào ao ICAMM originalmente proposto.

Consequentemente. no modelo ElCAMM, as matrizes de bases $\mathbf{A}_{k}$ são ortogonalizadas em cada itcraçào. utilizandu a scguinte equação:

$$
\mathbf{A}_{k}=\mathbf{A}_{k}\left(\mathbf{A}_{k}^{T} \mathbf{A}_{k}\right)^{1 / 2} .
$$

\subsection{Incorporação de Informações de Segunda Derivada}

Quando a segunda derivada da função objetivo é relativamente fácil de calcular, pode-se incorporar essa informação para acelerar a convergência do algoritmo c garantir a aproximação do mínimo local da funçào (Masters 1995). Com base nessa motivação, uma modificaçāo na regra de atualizasão para as matrizes de bases $\mathbf{A}_{k}$ foi proposta neste trabalho por meio da incorporação da segunda derivada da função de log-verossimilhança dos dados. A seguir, scrá 
apresentado como os métodos de Newton e Levenberg-Marquardt podem ser formalizados para serem utilizados no EICAMM.

\subsubsection{Método de Newton}

Para modelar o mét odo de Newton para o EICAMM, as segundas derivadas da função de log-vcrossimilhança, dadas perla matriz Hessiana, devem ser incorporadas à regra de aprendizado da Equação (7.4). Consequentenrente, a nova regra de atualização para as matrizes de bases $\mathbf{A}_{k}$ é dada por:

$$
\Delta \mathbf{A}_{k} \times p\left(C_{k} \mid \mathbf{x}_{t}, \boldsymbol{\theta}\right) \mathbf{H}^{-1} \mathbf{A}_{k}\left(\mathbf{I}-\mathbf{K} \tanh \left(\mathbf{s}_{k}\right) \mathbf{s}_{k}^{T}-\mathbf{s}_{k} \mathbf{s}_{k}^{T}\right)^{T} .
$$

tendo sido calculada. neste trabalho, por meio das seguintes derivaçōes:

$$
\Delta \mathbf{W} \propto\left[\mathbf{I}-\mathbf{K} \tanh (\mathbf{u}) \mathbf{u}^{T}-\mathbf{u u}^{T}\right] \mathbf{W}
$$

Calculando a segunda derivada em relação à $\mathbf{W}$ na Equação (7.7), obtém-se:

$$
\left(\mathbf{I}-\mathbf{K} \tanh (\mathbf{u}) \mathbf{u}^{T}-\mathbf{u} \mathbf{u}^{T}\right)+\frac{\partial\left(\mathbf{I}-\mathbf{K} \tanh (\mathbf{u}) \mathbf{u}^{T}-\mathbf{u} \mathbf{u}^{T}\right)}{\partial \mathbf{W}} \mathbf{W}=
$$

$\left(\mathbf{I}-\mathbf{K} \tanh (\mathbf{u}) \mathbf{u}^{T}-\mathbf{u} \mathbf{u}^{T}\right)+\left(-\mathbf{K} \tanh (\mathbf{u}) \mathbf{s}^{T} \mathbf{A}^{T}-\mathbf{K} \operatorname{scch}^{2}(\mathbf{u}) \mathbf{A} \mathbf{s u}^{T}-\mathbf{u} \frac{\partial \mathbf{u}^{T}}{\partial \mathbf{W}}-\frac{\partial \mathbf{u}}{\partial \mathbf{W}} \mathbf{u}^{T}\right) \mathbf{W}=(7$

$$
\left(\mathbf{I}-\mathbf{K} \tanh (\mathbf{u}) \mathbf{u}^{T}-\mathbf{u} \mathbf{u}^{T}\right)-\mathbf{K} \tanh (\mathbf{u}) \mathbf{u}^{T}-\mathbf{K} \operatorname{sech}^{2}(\mathbf{u}) \mathbf{u} \mathbf{u}^{T}-\mathbf{u A s} \mathbf{s}_{t} \mathbf{W}-\mathbf{A} \mathbf{s}_{t} \mathbf{u}^{T} \mathbf{W}
$$

Usando o resuitado $\mathbf{u}_{t}=\mathbf{W} \mathbf{x}_{t}-\mathbf{W A s _ { t }}$ c substituindo-o na Equação $(X)$, obtém-se:

$$
\begin{gathered}
\mathbf{I}-\mathbf{K} \tanh (\mathbf{u}) \mathbf{u}^{T} \cdots \mathbf{u} \mathbf{u}^{T}-\mathbf{K} \tanh (\mathbf{u}) \mathbf{u}^{T}-\mathbf{K} \operatorname{sech}^{2}(\mathbf{u}) \mathbf{u} \mathbf{u}^{T}-3 \mathbf{u} \mathbf{u}^{T}= \\
\mathbf{I} \cdots 2 \mathbf{K} \tanh (\mathbf{u}) \mathbf{u}^{T}-\mathbf{K} \operatorname{sech}^{2}(\mathbf{u}) \mathbf{u} \mathbf{u}^{T}-3 \mathbf{u} \mathbf{u}^{T}
\end{gathered}
$$

\subsubsection{Método de Levenberg-Marquardt}

Por meio da incorporação do método de Levenberg-Marquardt à regra para atualização para as matrizes $\mathbf{A}_{k}$, uma outra modificação também foi proposta, no modelo EICAMM, para garantir que a matriz Hessiana $\mathbf{H}$ scja positiva definida, uma vez que essa é uma condição necessária para que H seja inversível (Bazaraa 1979). Essa modificação é formulada como: 


$$
\Delta \mathbf{A}_{k} \propto p\left(C_{k} \mid \mathbf{x}_{t}, \boldsymbol{\theta}\right)(\mathbf{H}+\mu \mathbf{I})^{-1} \mathbf{A}_{k}\left(\mathbf{I}-\mathbf{K} \tanh \left(\mathbf{s}_{k}\right) \mathbf{s}_{k}^{T}-\mathbf{s}_{k} \mathbf{s}_{k}^{T}\right)^{T},
$$

onde $\mu$ é uma pequena constante no intervalo $(0,1)$.

Nos experimentos deste trabalho, o modelo EICAMM utiliza o método de LevenbergMarquardt em sua regra de aprendizado para as matrizes $\mathbf{A}_{k}$, como formalizado na Equação (7.12).

\subsection{Considerações Finais}

Neste capítulo, foi proposto o Modelo EICAMM, método auto-organizável derivado a partir de modificaçoes incorporadas ao modelo ICAMM, apresentado no Capítulo 6.

En experimentos apresentados no Capítulo 8, é demonstrado como o modelo proposto pode ser aplicado com succsso para classificação não supervisionada de conjuntos de dados simulados e o conjunto Iris.

Como o enfoçue deste trabalho consiste na aplicação de modelos auto-organizáveis para scgmentação de imagens. o EICAMM é aplicado em experimentos para segmentar imagens em suas versōes originatis c em versões pré-processadas pela metodologia proposta no Capítulo 5. 


\section{Capítulo 8}

\section{Resultados Experimentais}

\subsection{Consideraçōes Iniciais}

Neste capitulo, sato apresentados e analisados os resultados experimentais realizados no decorrer deste trabalho.

Inicialmente, o desempenho do método EICAMM, proposto no presente trabalho, é avaliado em tarefas de elassificaçăo nào supervisionada de dados simulados com distribuiços näo

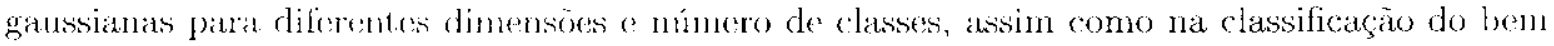
conhecido comjunto de dados lyis. Objetivando ainda uma discussäo mais abrangente, os resultados obtidos pelo método proposto é comparado com outros obtidos pelo método ICAMM na. sua forma original e pelo mótodo $k$-módias, para a classificação dos mesmos conjuntos do dados. Alguns resultades oftides por essá técnica também são apresentados por se tratar de um algoritmo clásico bastante utilizado cn análises comparativas envolvendo métodos auto-organizáveis.

Após essa anádise prolimina, sào apresentados uma série de resultados e discussöes cnvolvendo aplicaçoes para dados de imagens, uma vez que estes são os dados de interesse nesto traballo. Neste scntido, prinuriramente a metodologia de pré-processamento proposta no Capítulo 5 é avaliada quanto aos benefícios que esta pode gerar quando aplicada para pré-processar imagens a serem submetidas a rede neural Fuzyy ART para tarefas de segmentação. Finalmente. unificando as dus principais contribuiçoes originais desta tese, o método EICAMM é utilizado na segmentagào de inagens originais e das mesmas imagens pré-processadas pela metodologia propostia nester trablublio. 


\subsection{Experimentos com Dados Simulados}

Nesta seção. säo apresentados alguns resultados experimentais obtidos polos métodos EICAMM, ICAMM e k-médias para a classificagào näo supervisionadia de dades simulados, on seja, gerados artificialmente.

\subsubsection{Descrição dos Dados Simulados}

O ICAMN e, consequentemente o EICAMM. foram teoricamente formulados para realizar a classificaçäo de dados com distribuiçōes náa gaussianas. Dessa forma, com o objetivo de realizar una avaliação inicial do desempenho desses métodos. formu garados artificialmente alguns conjuntos formados por classes de dados com distribuiçoes uniforme (sub) gaussiana) e laplaciana (super-gaussiana).

Dessa forma, foram gerados, inicialmente 6 (seis) conjuntos de clados simulados com $N=2, N=3$ e $N=5$ dimensões $\mathrm{K}=2$ classes laplacianas (super-ganusianas), como pode ser visto na Figura 8.1 para o caso bidinensional. Para testar o caso no qual $k=3$, foi adicionada aos conjuntos de dados previamente gerados mais uma classe, desta vez com distribuiça uni.forme (sub-ganussiana), como pode ser visto na Figura 8.2 patra o cass bidimensional. As selas apresentadas nas Figuras 8.1 e 8.2 representam os vetores-colund dits matrizes de bases $A_{k}$ para cada clasise. Nesses experimentos com dados simulados, foram gerados 1000 pontos de dados para cada classe, embora somente 100 tenham sido utilizados para gerar as Figuras 8.1 e 8.2.

Uma ver que, neste caso dos dados simulados, os verdadeires rótulos dos padróes no conjunto cstão disponíveis, estes podem ser comparados con aqueles estimados pelos métodos, com o objetivo de realizar uma estimativa do ero de classificacaio comctido por cada modelo. Deve-se ressaltar, no entanto, que esse tipo de avaliação supervisionada foi feita apenas para so ter uma idéia inicial do desempenho do modelo EICAMM em comparação ans outros dois métodos, uma vez que o real interesse deste trabalho está relacionado ao caráter auto-organizável dos modelos aplicados.

\subsubsection{Resultados e Discussões}

O método de validaça cruzada con 10 partiçóes (10-fold) foi aplicado para estimar os erros verdadeiros obtidos pelos métodos EICAMM, ICAMM e k-médias na classificação dos dados simulados para $K=2$ e $K=3$ classes, apresentados na Ses, 8.2.1. Esses resultados podem ser vistos nat Tabela 8.1.

Em primeiro lugar, analisando o desempenho dos dois nodelos de misturas IC,A, pode-se 


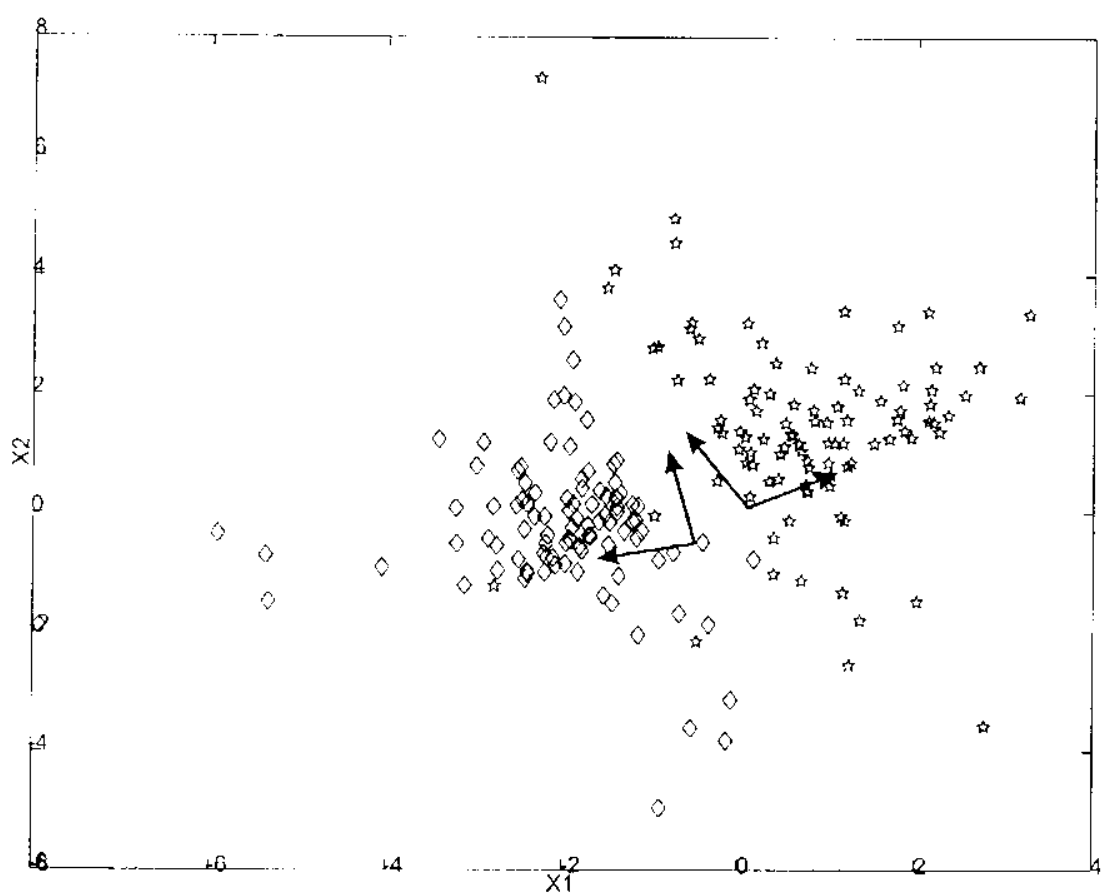

Figura 8. 1: Fxemplo de dados simulados com duas classes laplacianas.

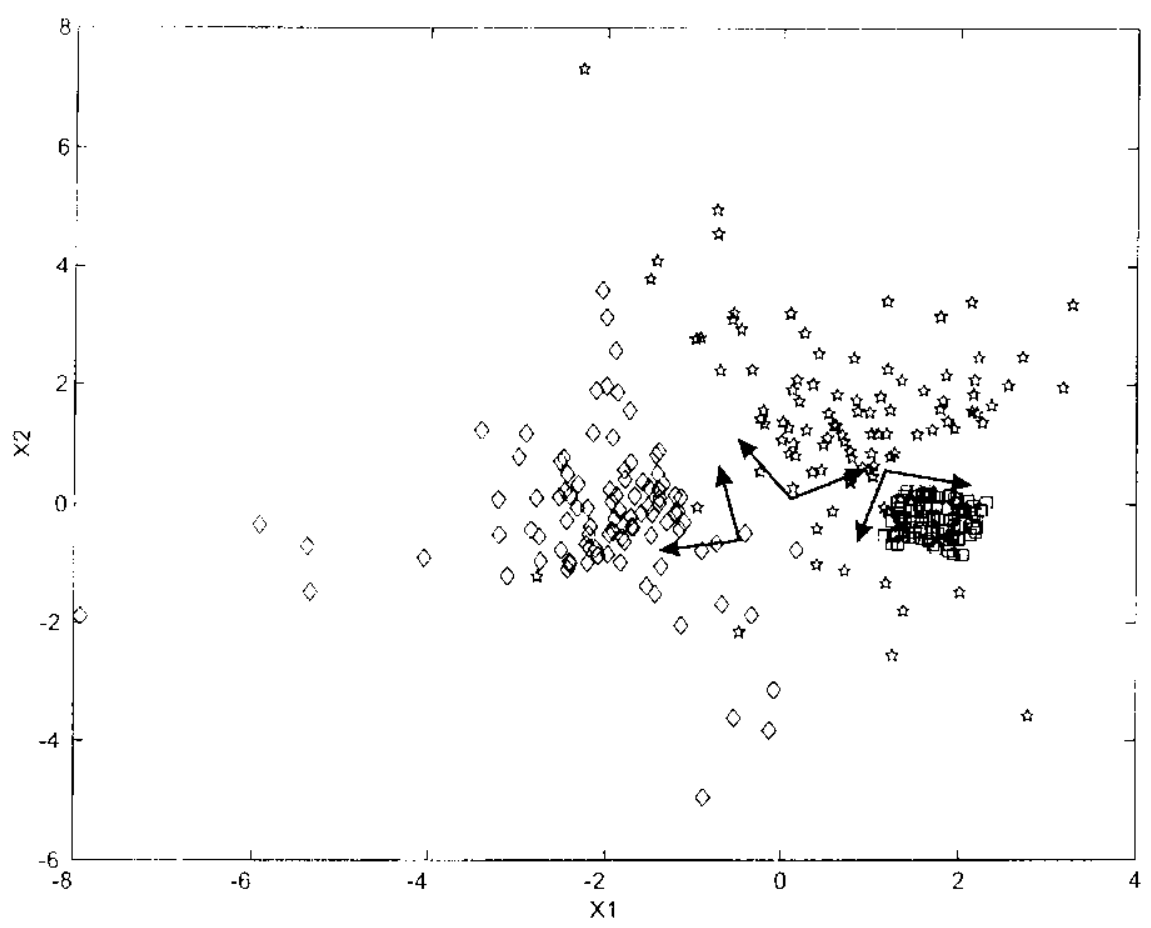

Figura 8.2: Vxemplo de dados simulados com duas classes laplacianas e uma classe uniforme. 
Tabcla 8.1: Resultados do classificação para darless simmlades.

\begin{tabular}{|c|c|c|c|c|}
\hline $\begin{array}{l}\text { Número } \\
\text { de Dimensōes }\end{array}$ & $\begin{array}{l}\text { Número } \\
\text { de Classes }\end{array}$ & $\begin{array}{l}\text { EICAMM } \\
\text { Erro Fstimado (\%) } \\
\text { média } \pm \text { desvio-padrão }\end{array}$ & $\begin{array}{c}\text { ICAMM } \\
\text { Erro Estimado (\%) } \\
\text { média : desvio-padrão }\end{array}$ & $\begin{array}{c}\text { k-médias } \\
\text { Frro Fstimado }(\%) \\
\text { média } \pm \text { desvio-padrão }\end{array}$ \\
\hline 2 & 2 & $8.85 \pm 2.64$ & $48.20 \pm 1.34$ & $1.75 \pm 2.50$ \\
\hline 2 & 3 & $15.52 \pm 3.68$ & $66.17 \pm 1.81$ & $28.10 \pm 2.21$ \\
\hline 3 & 2 & $7.92 \pm 2.52$ & $48.00+1.000$ & $5.75+1.82$ \\
\hline 3 & 3 & $20.40 \pm 6.56$ & $65.80+1.89$ & $33.26 \pm 9.21$ \\
\hline 5 & 2 & $8.75 \pm 3.40$ & $16.60 \pm 2.12$ & $2.55 \pm 1.21$ \\
\hline 5 & 3 & $32.95+3.07$ & $65.99+1.03$ & $49.80 \pm 2.31$ \\
\hline
\end{tabular}

notar que os resultados de classificação obtidos pelo EICAMN supetaram, de forma significativa, aqueles obtidos pelo método ICAMM, que por sua vez mostron-se ineficiente em todos os experimentos com dados simulados realizados neste trabalho. Como observado no decorrex dos experimentos. essa ineficiência deve-se ao fato de que a tendencia do método ICAMM ć associar a uma só classe praticamente todos os dados do conjunto. mestno gue vários esquemas para definiçăo de taxas de aprendizado tenham sido tcstados.

Já em comparação ao k-médias; o FICAMM obteve resultados superiores em todos os casos em que o número de classes é igual a 3. Entretanto, o seu demenpenho foi inferior a $k$ médias nos experimentos envolvendo dados com duas classes. Isto nāo significa necessariamente (a depender, por exemplo, da complexidade dos dados e da aplicango) que o EACAMN näo posia ser considerado um bom classificador, uma vez que o erro verdadeiro estimado fica abaixo dos $10 \%$ nesses catsos. Inclusive, considerando o desempenho do EICAM isclarlamente, para duas classes o erro foi menor do que para três classes, como pode ser visto na Tabela 8.1. Para duas classes existem bons classificarlores descritos na literatura. () mainr problema em termos de classificaçäe ocorre para mais do que duas classes. Desta forma, nos experimentos de imagens destee trabalho buscou-se por resultados com múmero de classes maior do que 2 .

Para analisar visualmente como o modelo EICAMT a o algoritmo k-médias podem se auto-organizar para detectar agrupamentos em um conjunto do diados, a scoguir são considerados outros resultados envolvendo 2 classes de diados super ganssianos geradats artificialmente.

\subsubsection{Análise Visual de Resultados}

O comportamento auto-organizável dos métodos FICAMM c k-médias para dados superganussianos simulados foi avaliado neste trabalho por meio da análise visuat envolvendo os rótulos verdadciros dos dados de um conjunto e aqueles encontrados pelas duas abordagens. Para isso foi gerado um outro conjunto com duas classes laplacianas. $A$ escolla desse tipo de distribuicáno 
nessen experimentos deve-se ao fato de que essa distribuiça é frequentemente adequada para descrever dados de imagens (ver (apítulos 4 e 5), que são os dados de maior interesse para o prescinte trabalho.

Para proceder a rise experimento, foram gerudes 1000 cxemplos para cada classe, totalizando 2000 pontos de dados no conjunto. Os dados foram aleatoriamente divididos em um conjunto de treinamento, contendo $90 \%$ dos dados e outro de teste, composto pelos $10 \%$ dos dados restantes. Os dados desse conjunto de teste foram rotulados com as suds verdadeiras classes e podern ser vistos na Figura 8.3(a). Os dados rotulados com as classes encontradas pelo EICAMM e pelo $k$-módias são apresentados nas l'iguras $8.3(\mathrm{~b})$ e $8.3(\mathrm{c})$, respectivamente. A inversäo dos rótulos encontrados pelos dois métodos deve-se, nesse caso, à natureza autoorganizável das duas técnicas, que nảo utilizam en seus algoritmos informaçoes sobre as classes verdadeiras.

Pode-se observar que os dois métodos conseguiran se auto-organizar para detectar corretamente os rótulos da grande maioria dos pontos de dados. Entretanto, ambas as técnicas falharam na classificação de dados localizados em áreas de sobreposição e em coordenadas afastadas dos pontos de maior concentração das classes.

\subsection{Experimentos com o Conjunto de Dados Iris}

O objetivo dos experimentos descritos nesta seção é avaliar o desempenho do modelo EICAMM, em comparaçà̃o com o modelo ICAMM e o algoritmo $k$-méclias, na classificação de conjuntos de dados para os quais não se conhece de antemäo as distribuiçoes de probabilidade das classes. Para isso, resolveu-se utilizar o já bem conhecido conjunto de dados Iriś, que será brevernente descrito a seguir.

\subsubsection{Descrição dos Dados}

O conjunto de dados Iris (ver, por exemplo, (Duda \& Hart 1973)) contém três classes que representam tipos de flores, com 50 exemplos em cada urnat, e quatro atributos numéricos. Uma dass classes é linearmente separável das outras duas, as quais não sāo lincarmente separáveis entre si. Para maiores detalhes, esse conjunto de dados encontra-se disponivel no repositório UCI (Blake \& Merz 1998). 


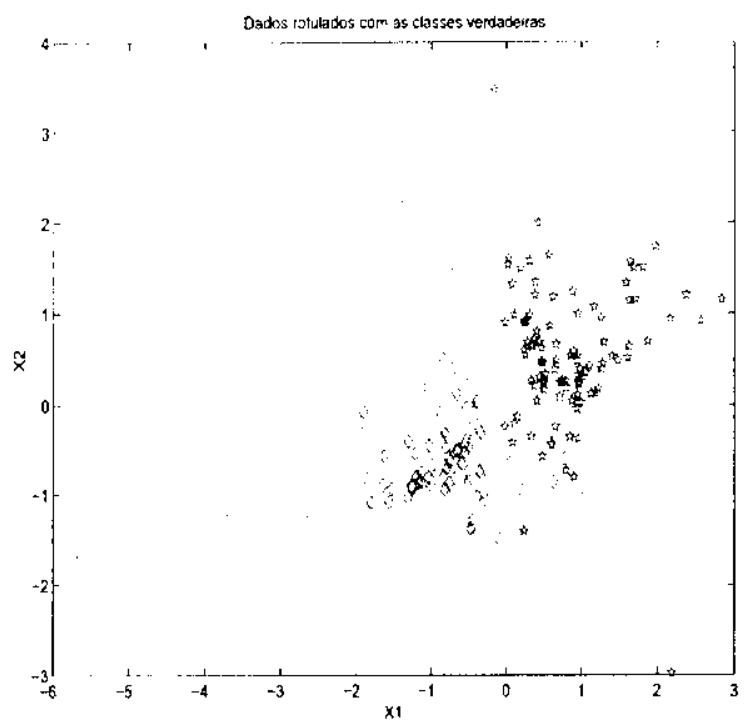

(a)

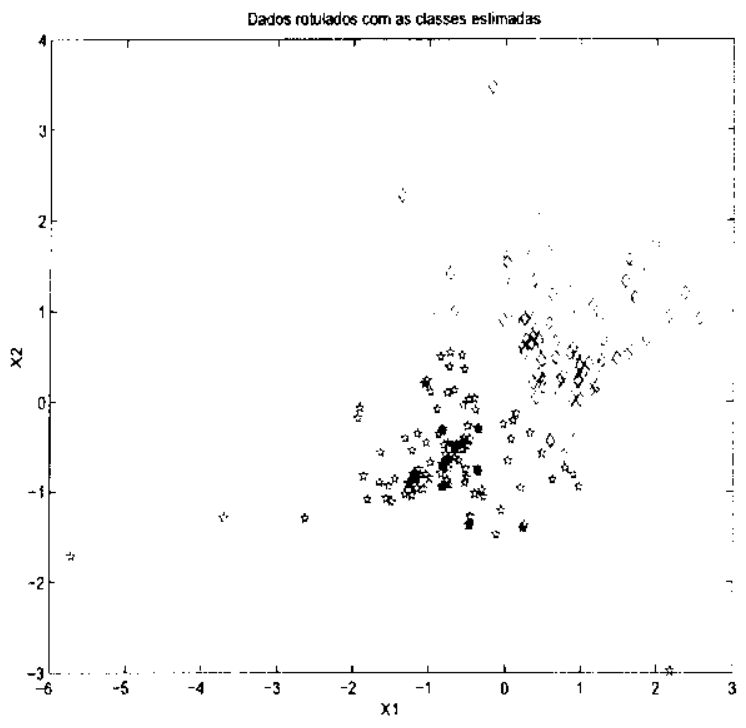

(b)

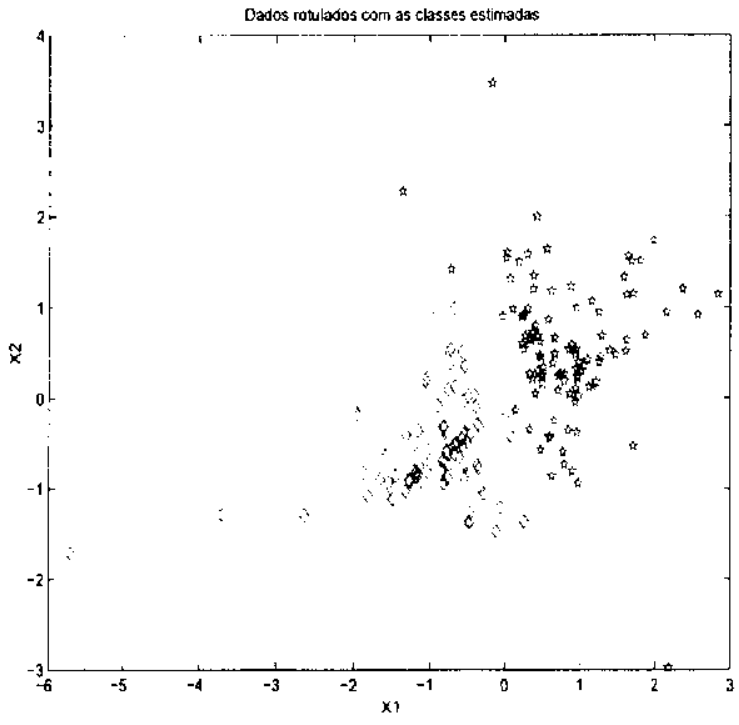

(c)

Figura 8.3: Análise visual de resultados dos métodos EICAMM e $k$-médias. (a) Visualização de dados simulados com duas classes laplacianas. Neste caso, os dados estão rotulados com as suas verdadeiras classes; (b) Visualização de resultados obtidos pelo EICAMM. Neste caso, os dados estão rotulados com as classes estimadas pelo modelo; (c) Visualização de resultados obtidos pelo $k$-médias. Neste caso, us dados estão rotulados com as classes estimadas pelo método.

\subsubsection{Resultados e Discussōes}

O método de validação cruzada com 10 partiçōes (10-fold) foi aplicado para estimar os erros verdadciros obtidos pelos métodos EICAMM, ICAMM e k-médias na classificação dos 
datos Iris. Essers resultardos porken ser vistos na Tabela 8.2.

Talyela 8.2: Resultados do elassilicagano parad o conjunto de dados Iris.

\begin{tabular}{|c|c|c|}
\hline $\begin{array}{c}\text { WICAMM } \\
\text { Erro Estimado (\%) }\end{array}$ & $\begin{array}{c}\text { ICAMM } \\
\text { Erro Estimado (\%) } \\
\text { média } \pm \text { desvio-padräo }\end{array}$ & $\begin{array}{c}\text { E-médias } \\
\text { Erro Estimado (\%) }\end{array}$ \\
\hline $30.42+3.58$ & $61.99 \pm 10.90$ & $36.66 \pm 7.97$ \\
\hline
\end{tabular}

Polv-so motar que os modelos utilizados apresentaran desempenhos semelhantes aos observados na classificacão dos dados simmlados, para o caso de $K=3$ classes. Novamente, os resultados obtidos pelo FICANM superaram significativanente aqueles obtidos pelo ICAMM. senclo também superiones aos alcangados pelo algoritmo k-médias. Entretanto. para os dados simmlatos e conjunte) lris existem algumas limitaşôs relativas à aplicação do EICAMM. A primeira delas é que o método, embora aprescnte uma convergenecia más rápida do que a apresentada pelo ICAMM (que näo comvergiu antes de 10000 iterascos cm nemhum caso), necessita anda de mu grande número de iteracoes, que para esses conjuntos de dados ficaram em torno de 2000 itregeons para carla partiga (fold) analisada. Além disso, a cada novo conjunto aprosentado ao modelo a taxa do aprendizado deve ser ajustada empiricamente. Para esses experinentos. us valores para esses parâmetros variaram critre 0.00001 e 0.001 .

Em (Oliveia s Romero 2004a), uma comparação entro os resultados obtidos pelo EICAMM e pelo 1CA.MM para a classificagăo mo supervisionada dos dados Iris e de dados simulados, semelhintos aos apresentartos anteriormente neste capítulo, serviram como base para a validação do modelo EICAMM, proposto neste trabalho.

\subsection{Experimentos com Imagens}

Nesta sera, os experimentos com imagens realizados no presente trabalho são descritos e discutidos. Os principais resultados avaliados aqui dizem respeito à utilização da metodologia proposta para péprocessamento de imagens, descrita no Capítulo 5 e a aplicaço de nótodos

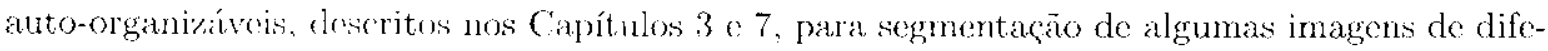
rentes domínios, descritas logo a seguir.

\subsubsection{Descrição dos Dados de Imagens}

Foram utilizadas nos experimentos desta seção quatro imagens de diferentes domínios, das quais alguns cletalhes envolvendo suas descriçoes säo apresentados na Tabela 8.3 . 
Tabela 8.3: Detalhes de descrição das imagens utilizadias nes experimentos.

\begin{tabular}{|c|c|c|c|}
\hline Imagem & $\begin{array}{l}\text { Número } \\
\text { de Tinhas }\end{array}$ & $\begin{array}{l}\text { Número } \\
\text { de Colunas }\end{array}$ & Fonte \\
\hline I1 & 256 & 512 & http://www.cis.hut.fi/projects/ica/data/image \\
\hline $\mathrm{I} 2$ & 512 & 768 & ftp://ipl.rpi.edu/pub/image/still/Kodakimages/ \\
\hline I3 & 512 & 512 & http://vision.ece.ucsb.edu/data_hiding/ETlena.html \\
\hline $\mathrm{I} 4$ & 512 & 640 & Departamento de Fitursuidade UNESP - Jaboticabal \\
\hline
\end{tabular}

A imagem denotada aqui por I1 faz parte de urn conjunto de inagens de cona natural (ver Figura 8.4(a)); a imagem 12 faz parte de um conjunto de imagens demonstrativas do sistema PhotoCD da Kodak (ver Figura 8.4(b)); a imagem I3 é uma imagem conhecida como Jena, amplamente utilizada na literatura de processamento de imagens, por ser de domínio público há vários anos (ver Figura $8.4(\mathrm{c})$ ); o a imagem I4 foi cedida para os cxperimentos reste trabalho pelo Departamento de Fitossanidade - UNESP - Jaboticabal. Tal imagem foi obtida por microscopia realizadi erm plantas para deteção de esporos, denominados de ascópuros.

f́. importante observar que, apesar das imagens terem sido processadas nos experimentos em seus tamanhos originais, aqui foi fcita uma padronização das suas dimensoos (número de

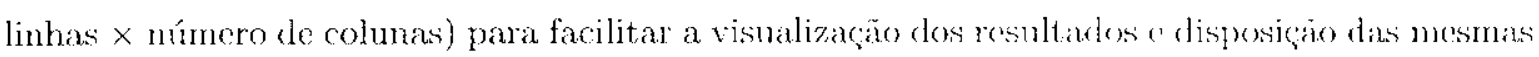
ao longo do texto.

\subsubsection{Aplicação da Metodologia de Pré-Processamento Proposta}

Os experimentos descritos nesta subsegato dizem respeito à aplicaysa da metodologia de pré-processamento de imagens, proposta no Capítulo 5, às imagens originais apresentadas na Figura 8.4. Nessa abordagem, o método SCS e o detector de bordits de Sobel säo combinados para construir uma imagem que apresente caracteristicas mais adequadas fara um processo de segmentação. P'rimeiramente, o método SCS é aplicado com as lina lictades de reduçäo de ruídos e suavização da imagem original, tomando as áreas de maior interosio mais homogèneas. O resultado desse primeiro estágio é, entăo, combinado com a intagem vesultante da aplicaçáo do detector de bordas de Sobel à imagem original, com a finalidade de ressattar bordas importantes.

Os resultados desses experimentos para pré-processamento podem ser visualizados na Figura 8.5. Se comparadas com as imagens originais apresentadas na Figura 8.4 a possivel notar que as imagens pré-processadas apresentam uma maior homogeneidade nas regiōos que definem os mesmos objetos on texturas, como é o caso. por exemplo, daw folltagenss na imagem I], portas e janelas na imagem I2, regiōes de tcxtura de pelc na imagen I3 e cstruturas microscópicas wa imagem I4. Além disso, em todos os resultados, observa-se a reducio do bribo presente nas 


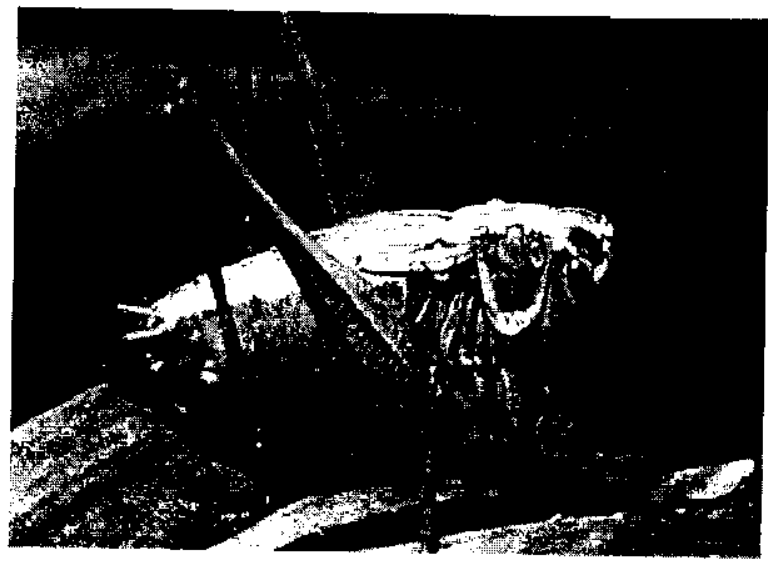

(a)

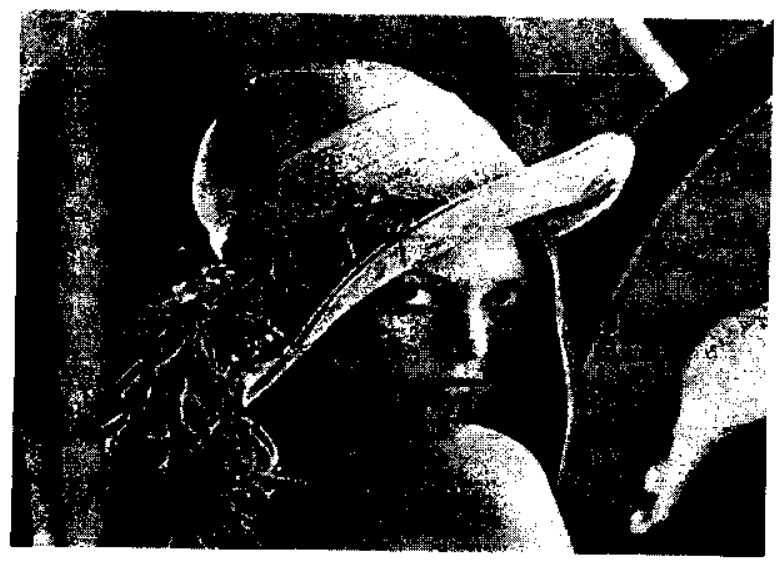

(c)

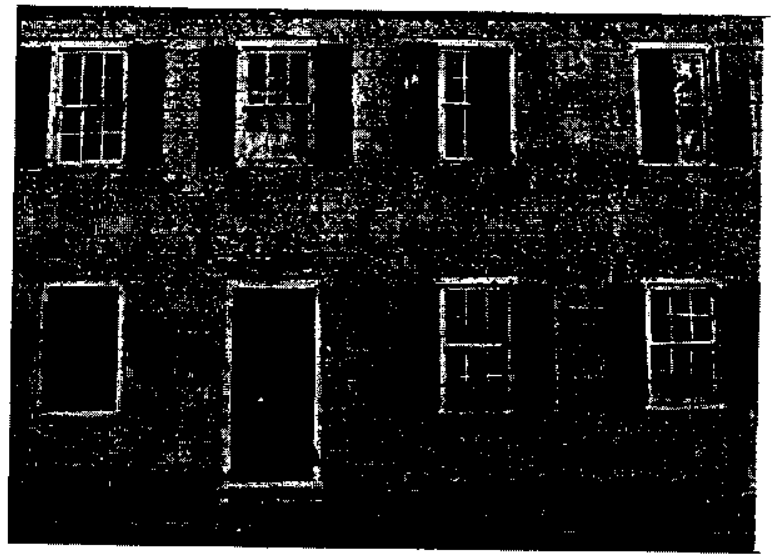

(b)

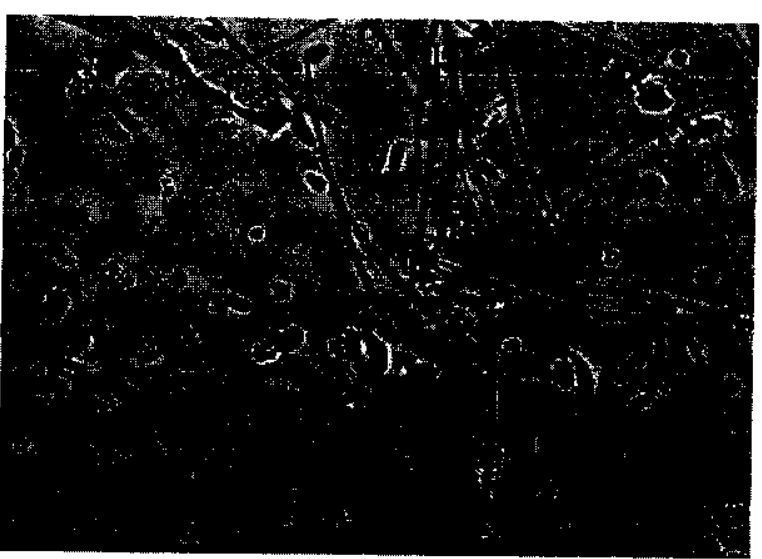

(d)

Figura 8.4: Imagens originais utilizadas nos experinentos. (a) Inagem 11; (b) Imagem I2; (c) lmagen I3; (d) Imagem I4.

imagens originais. A redução desse fator também é importante, uma vez que à presença de brilho excessivo on uma imagem pode prejudicar o resultado de segmentação da mesma.

Em todos esses experimentos, o nível (variância) do ruído estimado foi fixado em 0.1. Observou-se que o valor clesse nivel é diretamente proporcional ao fator de suavizaçăo aplicado à indagem original. Dessa forma, quanto major o nível do ruído estimado (definido pelo usuário) major scrá à suavização applicada à imagem.

É importante ressaltar que não foi considerado nos experimentos deste trabalho a adição proposital de ruídos nas imagens, nem a necessidade em se conhecer de antemão se uma determinada imagen apresenta ou não ruído. Dessa forma, o termo ruído aqui é considerado 


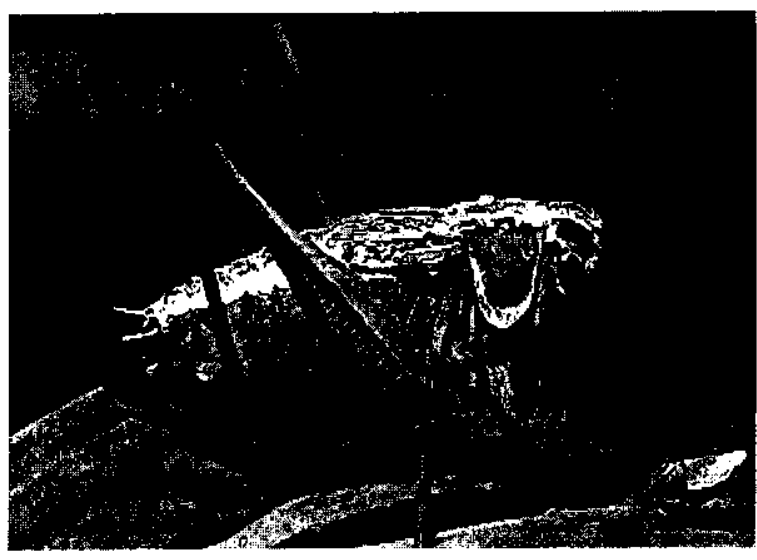

(a)

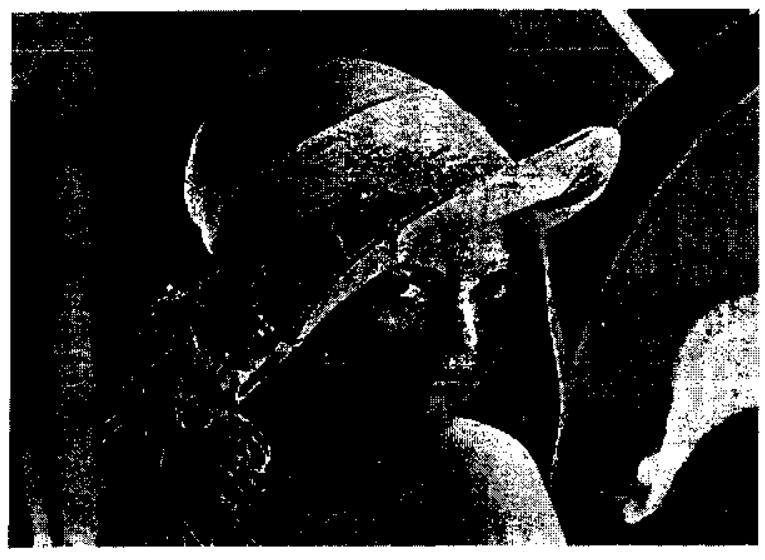

(c)

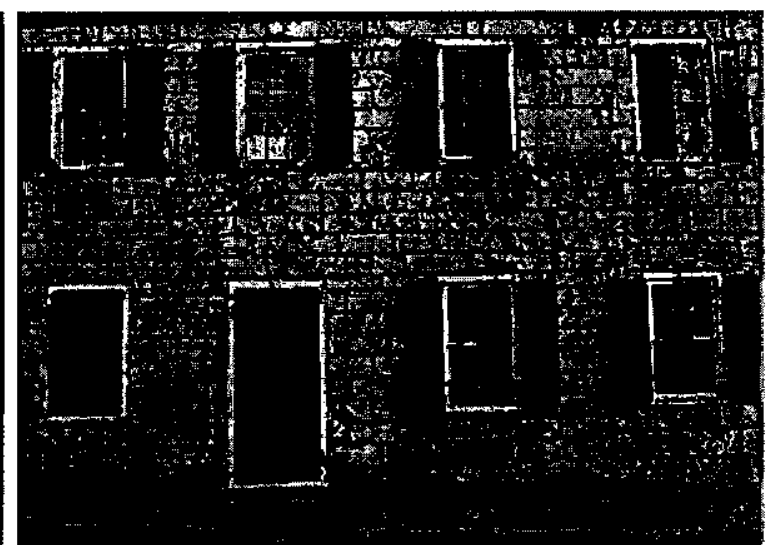

(b)

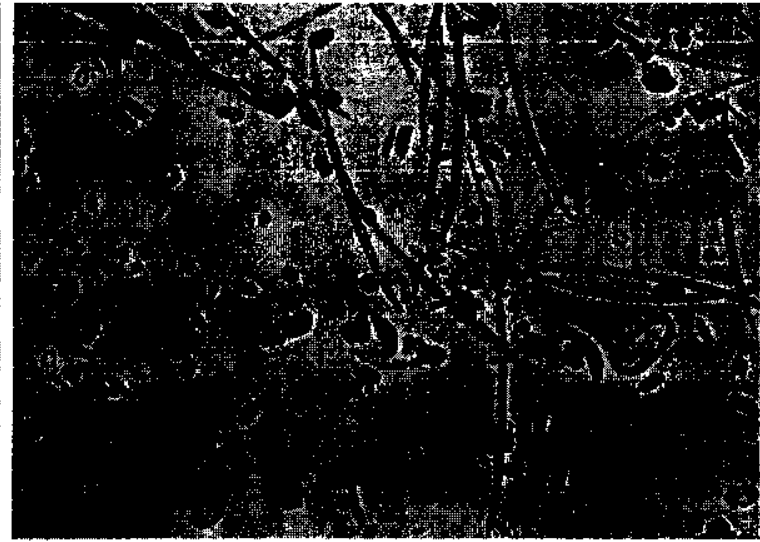

(d)

Figura 8.5: Imagens resultantes da aplicação do método de pré-processantento propesto, que combina o método SCS co detector de arestas de Sobel

como qualquer pequeno objeto ou variaçoes irrelevantes que pessim prejudicar o resultado de segmentação de uma imagem.

O detector de arestas de Sobel também possui um papel imnomtante na abordagem de préprocessamento, uma vez que este é utilizado para auxiliar na recomposição das bordas borradas no processo de suavizaçào, podendo restaurar, então, alguns linites cutire os objetos de interesse cm urna irragem.

Como será visto a seguir, a aplicarção dessa metodologia de pré-processumento proposta de fato acarroton em melhorias nos resultados de segmentação obtidos polos modelos autoorganizáveis apresentados neste trabalho. 


\subsubsection{Experimentos de Segmentação de Imagens}

Nesta sulberăo, os modelos auto-organizáveis Fuzzy $\Lambda$ RT, EICAMM e o algorit mo kmédias são avaliados quanto aos seus desempenhos no processo de segmentação das imagens escollhidas para análise.

Primeiramente: 6́ necessário explicar como os dados de imagens foram formatados a fim de serem procossardos pelos métodos avaliados. Nessa etapa, procedeu-se da seguinte forma: para cada pixel da imagem for construído um vetor de níveis de cinza, composto pelo valor desse pixel. como clemento central. c: dos seus oito vizinhos mais próximos em uma vizinhança quadrada. Para ilustrar esse esquema, o padräo de entrada relativo ao pixel $x_{5}$ apresentado na Figura 8.6 a compesto como o votor de 9 dimensöes $\left(x_{1}, x_{2}, x_{3}, x_{4}, x_{5}, x_{6}, x_{7}, x_{8}, x_{4}\right)$, onde os valores de $x$ a thisumen os valores dos níveis de cinza daqueles pixels. Para tornar esse esquema viável, os pixels das bordas das imagens foram descartados.

\begin{tabular}{|l|l|l|}
\hline$x_{1}$ & $x_{2}$ & $x_{3}$ \\
\hline$x_{4}$ & $x_{5}$ & $x_{6}$ \\
\hline$x_{7}$ & $x_{8}$ & $x_{9}$ \\
\hline
\end{tabular}

Figura 8.6: Esquema de formatação de padrōes para dados de imagens. Para cada pixel da imagem é construído nu vel or de níveis de cinza composto pelo valor desse pixel, como elemento central, e dos seus oito vizinhos mais próximos em uma vizinhança quadrada.

Como resultado do processo de segmentação, o pixel central de cadia padrão é rotulado com um nivel de cinza que identifica a classe a que este foi associado. Por exemplo, os nívcis de cinza 0 (preto) o 255 (branco) podem servir como rótulos em um esquema de segmentação envolvendo duas cliasses.

\section{Experimentos com a Rede Neural Fuzzy ART}

O primeiro modelo auto-organizável a ser aplicado à segmentaçăo de imagens neste trabalho foi a rede neural Fuzzy $A \mathrm{Rl}$, apresentadia no Capítulo 3. De fato, essa rede foi escolhida para testar o desemuento da metodologia de pré-processamento proposta. Essa escolha deveuse ao fato do que este modelo já havia sido implementado e analisado em (Vicentini 2002) c (Vicentini, Oliveira \& Ronnero 2004), onde foi feita uma avaliação para encontrar qual seria: dentre os modelos ART2, ART2-A c Fuzzy ART, o mais adequado para ser combinado com a estrutura de dados métrica Slim-Tree (Traina Jr., J. M. Traind, Seeger \& Faloutsos 2000). 
O objetivo final desses trabalhos foi a obtenção de um modelo híbrido para indexaçào e recuperação de informações, capaz de responder a consultas por similaridade. Para essa aplicaçäo, os modelos ART2 e ART'2- $A$ mostraram un desempenho superior em relação ao apresentado pola rede Fuzzy $\Lambda R T$.

No critanto, em experimentos preliminares com imagens realizados no prescnte trabalho, o modelo ART que apresentou resultados melhores foi o modelo liuzy ART. Esse modelo foi aplicado com sucesso, em combinação com o método Sparse Code Shrintage, para segmentação de irnagens obtidas por um robô móvel (Oliveira, Vicentin \& Romero 20102).

Considerando as imagens analisadas neste capítulo, os resultados de segmentação obtidos pela rede Fuzzy ART, para as versoes originais e pré-procosisadas wio os apresentados na Figuras 8.7 e 8.8, respectivamente. Nesse caso, o núnero máximo de agrupanuentos foi estipulado em $K=2$. N lóm disso. os parâmetros da rede foram: $x$-...0.0008. $\beta$ - 0.01 e $\rho$ o.80, lembrando que esse último parâmetro determina o grau de similaridade mínimo entre os padrös alocados em un mesmo cluster.

Comparando-se os resultados de segmentação obtidos pela rede Fuyzy ART para as imagens originais o pré-processadas, pode se notar que de fato. a metodologia de pré-processamento proposta neste trabalho leva a unia melhoria nos resultados finais do processos.

Entretanto, também foi observado que a segmentaçăo ralizarla pela rede Fuzzy ART, neste caso, detectou, basicamente, apenas os contornos des objetes presentes nas imagens, não sendo capar de definir algumas regiôes de interesse. Como un número de agrupamentos $K=2$ pode ser considerado perieno para a detecgăo de ostruturas básicas em imagens com relativa complexidade, como é o caso das imagens analisadas nesto trabaho. outos experimentos foram realizados para um número máximo maior de clusters e valores menores para o paràmetro de vigilância $\rho$. Ainda assim o número de clusters detectados pelo modelo não passon de 2 e resultados melhores do que os apresentados na l'igura 8.8 näo foram obtidos.

\section{Experimentos com o Modelo EICAMM}

Para avaliar a unificação das propostas originais deste trabslho, o método EICAMM foi aplicado para segmentação das imagens originais e pré-processadas. apresentadas nas figuras 8.4 e 8.5. Neste caso, considerou-se um número $K=3$ de agrupamentos. Os resultados obtidos são apresentados na Figura 8.9, para as imagens originais e na ligura 8.10. para a imagens pré-processadas pela metodologia propostiat.

O primeiro ponto a ser ressaltado na análise desses experimentos é que o modelo EICAMM, proposto neste trabalho, de fato é adequado paria clasificacia de dados de imagem, 


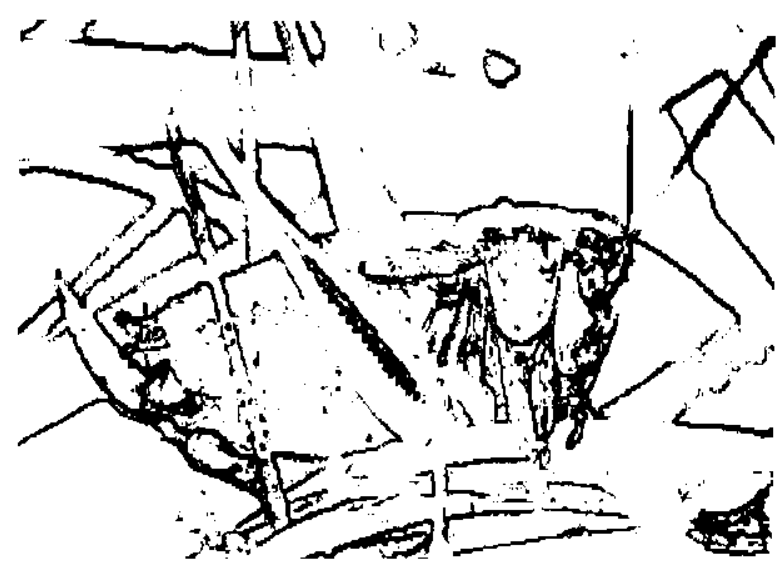

$(a)$

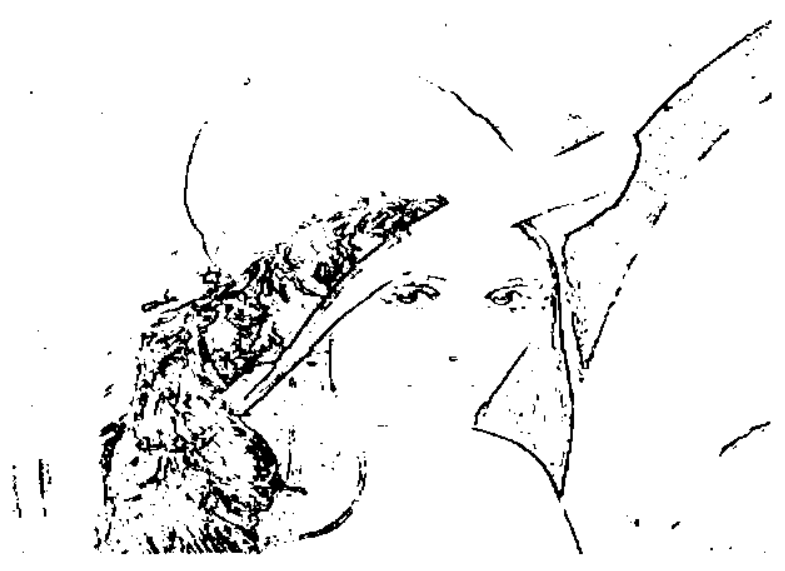

(c)

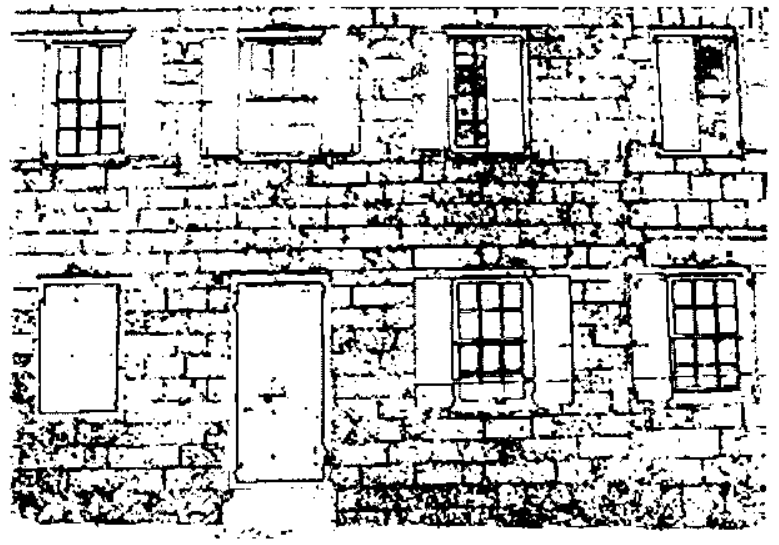

(b)

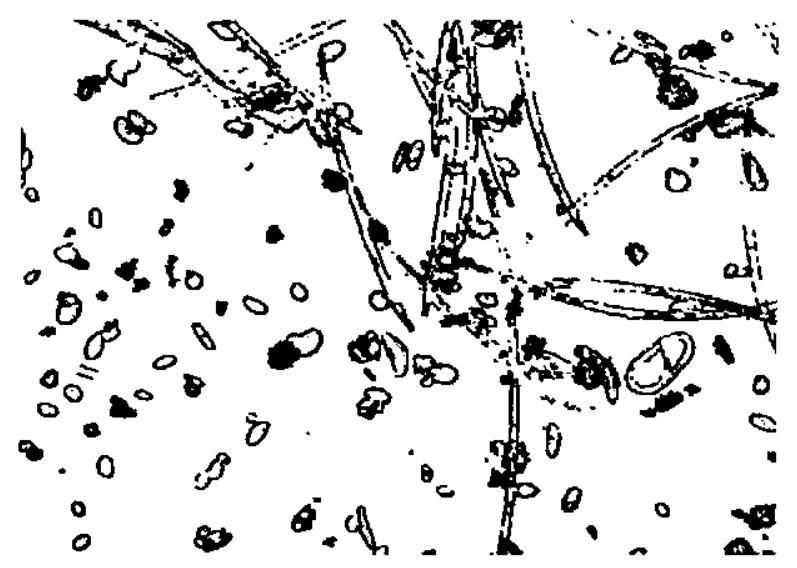

(d)

Figura 8.7: Resultados de segntentaçäo utilizando a rede neural Fuzzy $\Lambda R T$ aplicada às imagens originais. (a) resultato para intagem 11 ; (b) resultado para imagem 12 ; (c) resultado para imagem I3; (d) resultado para imagern 14 .

tendo obtidos resultados significativannente melhores do que aqueles obtidos pela rede neural Fuzzy ART, como pode ser observado nas imagens das Figuras 8.8 e 8.10. Pode-se notar, que ao contrário do modelo neudal, o EICAMM conseguiu detectar regiões homogêneas que representam estruturas (objetos) importantes presentes nas imagens. Alérn disso, assim como nos experimentos conn a rede Fuxy ART é possivel observar como a utilização da metodologia de pré-processamento propostia levou a resultados de segmentação significativamente melhores do que aqueles obticlos quanto a técnica é aplicada às imagens originais.

Em outros experinentos, a número de classes estimadas foi elevado para $K=5$. Es- 


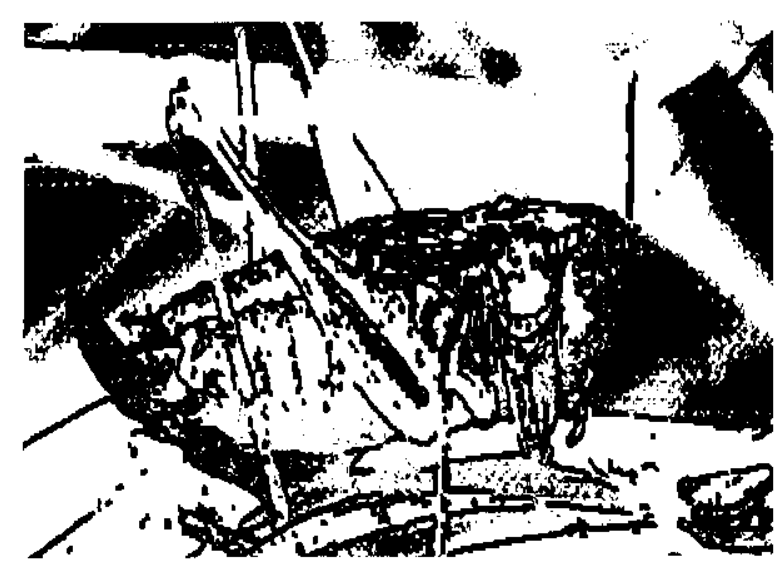

(a)

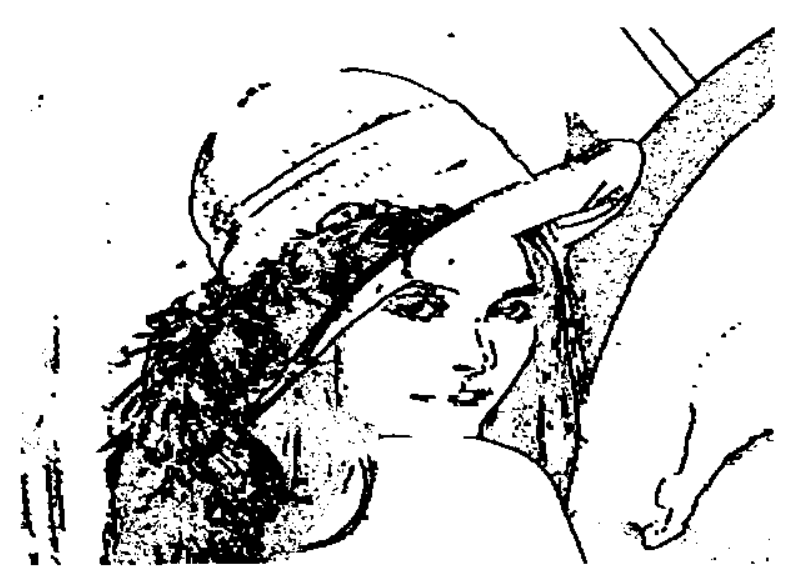

(c)

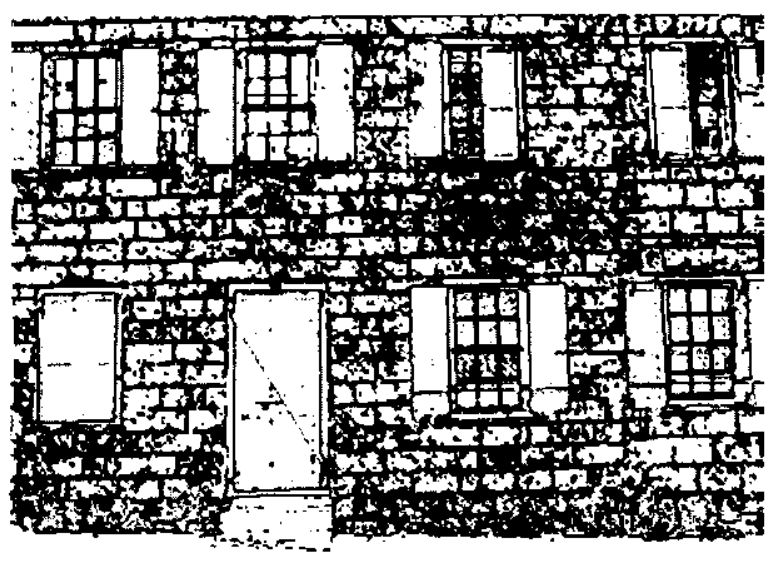

(1).)

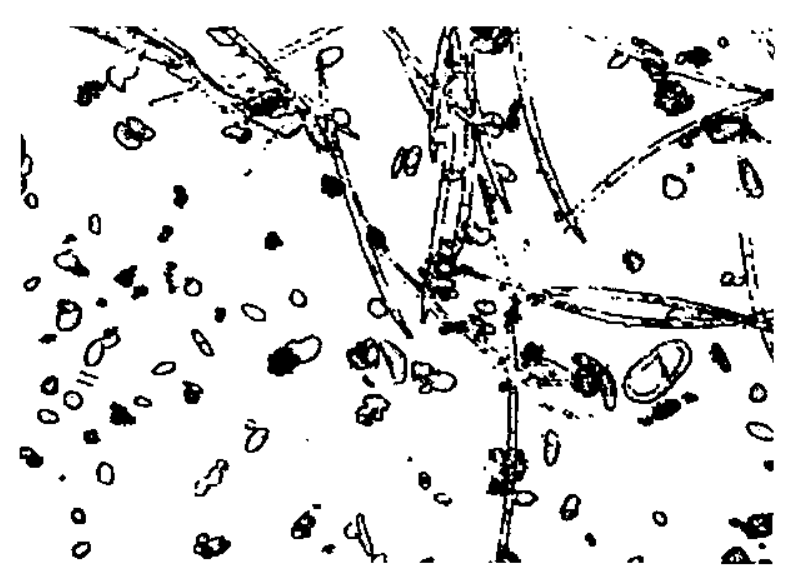

(d)

Figura 8.8: Resultados de segmentação utilizando a rede neural fuzzy AIRT aplicuda ds imagens pré-processadas. (a) resultado para imagem I1; (b) resultado para imagom I2; (c) resultado para imagem 13; (d) resultado para imagem 14

ses resultados são apresentados na Figura 8.11. Novamente. O FICAMM conseguiu realizar a segmentação das imagem pré-processadas, porém o aumento do múnero de classeses näa levon à resultados melhores do que no caso para $K=3$, como pode ser observado comparando-se as imagens das Figuras 8.10 e 8.11. Ao contrário, para algumas inaggens, como a imagem I3 a imagem $I 4$, os resultados foram piores do que os obtidos para $K=3$.

Un outro aspecto importante observado nos experimentos molvendo a aplicaça do EICAMM em dados de imagens é que, ao contrário do que foi notades nos experimentos com dados simulados e o conjunto Iris, o EICAMM apresentou uma convergencia relativamente 


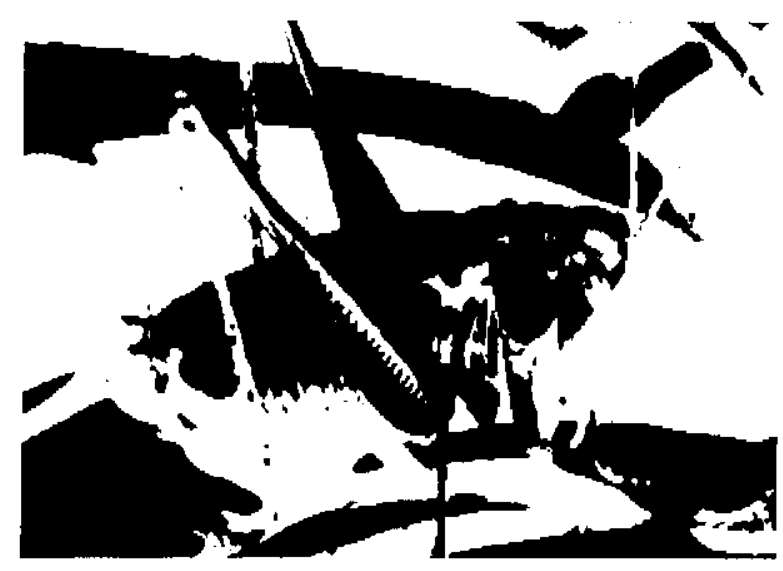

(a)

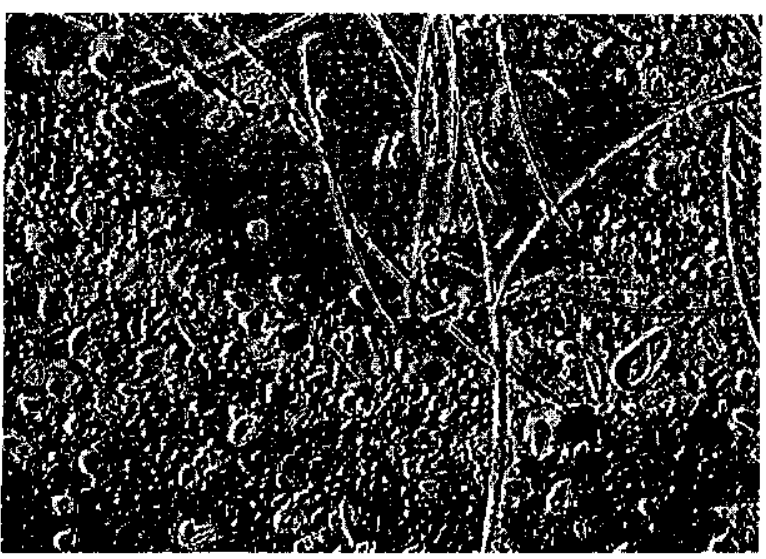

(c)

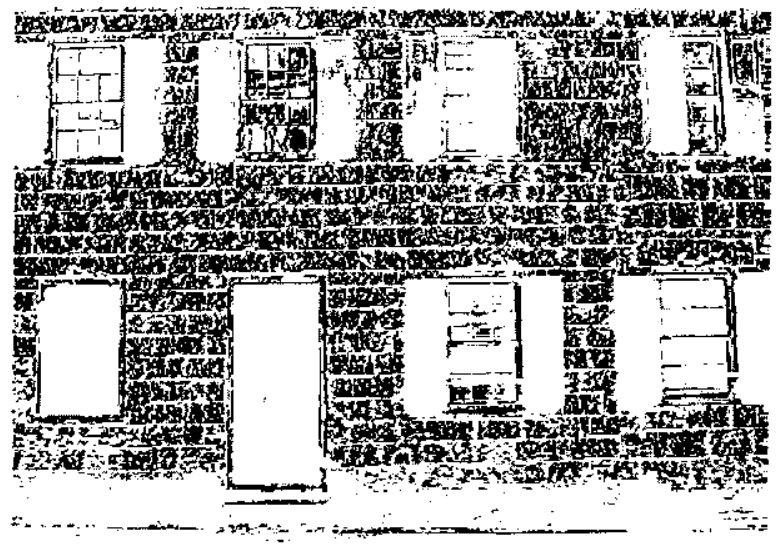

(b)

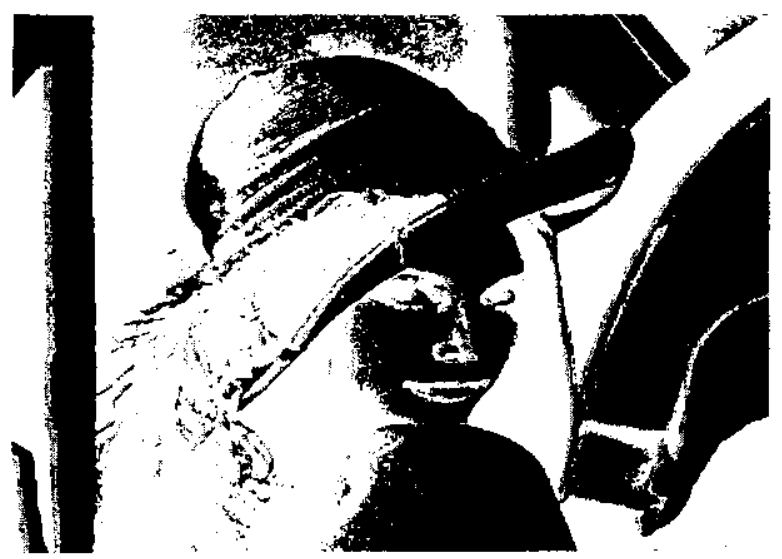

(d)

Figura 8.9: Resultados de segnnentação de imagens utilizando o modelo EICAMM aplicado às imagens originais. Todas inagens foram segmentadas pelo modelo EICAMM para $k=3$ classes. (a) resultado para imagem 11: (b) resultado para imagem I2; (c) resultado para imagem I3; (d) resultado para imagem 11.

rápida, com un numero de itcracōos abaixo de 100. Além disso, a taxa de aprendizado foi fixada em 0.000001 para todos as imagens, sem necessidade de ajustes. Esses pontos positivos da aplicaçăo do EICA.MM a dados de imagens vêm comprovar que de fato o método é adcquado para o processamento desse tipo de dado. 


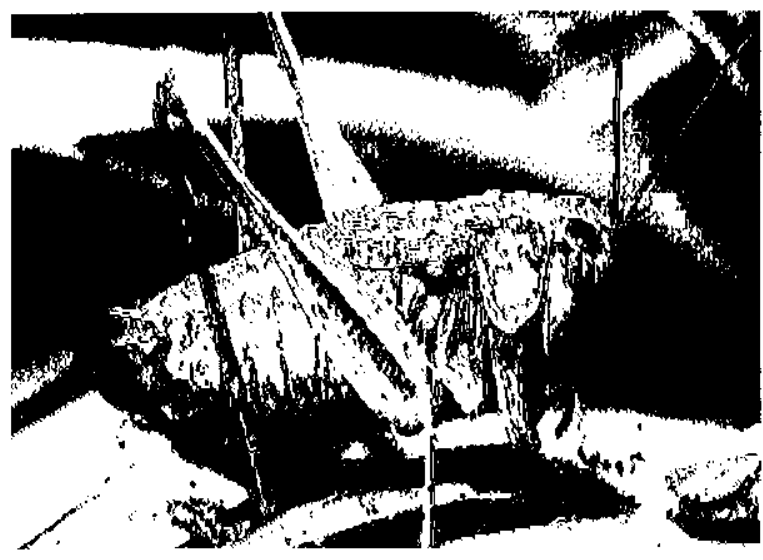

(a)

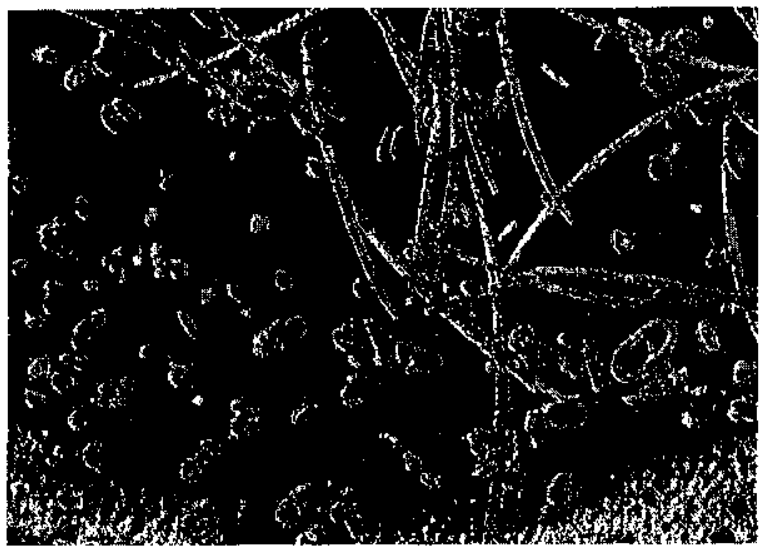

(c)

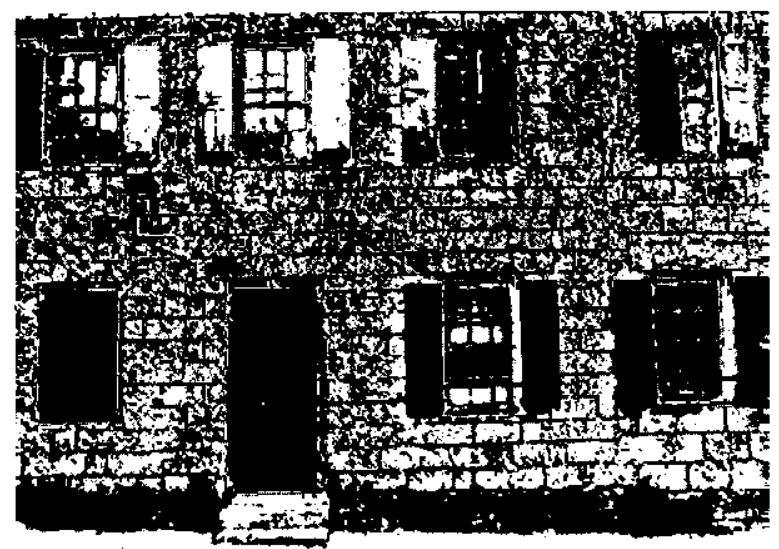

(b)

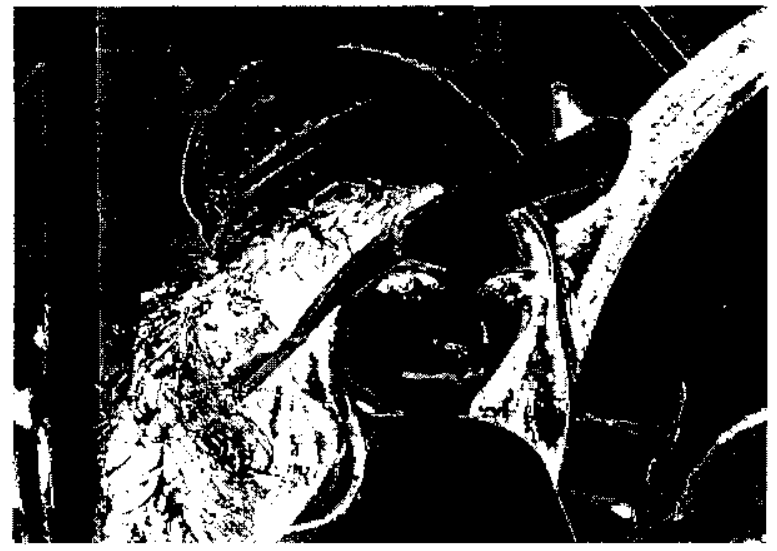

(d)

Figura 8.10: Resultados de segmentação de imagens utilizando o modelo ElCAMM aplicado às imagens pré-processadas. Todas imagens foram pré-processadas pela metodologia proposta c segmentadas pelo modelo EICAMM para $k=3$ classes. (a) multado para imagem I1; (b) resultado para imagem I2; (c) resultado para imagem I3; (d) resultado para imagem I4.

Experimentos com o Método k-médias

Para efeitos comparativos, alguns resultados obtides pelo método k-médias para segmentaçäo das imagens consideralas neste traballo săo apreseut ulos a seguir.

lima vez que a metodologia de pré-processamento foi validada nos experimentos com a rede Fuzzy ART e o modelo ĖCAMM, as imagens pré-processadas foran darlas cono entlatad para o algoritmo $k$-médias cm expcrimentos de segmentação para $K=3$ classes. Esses resultados podem ser vistos na ligura 8.12 . 


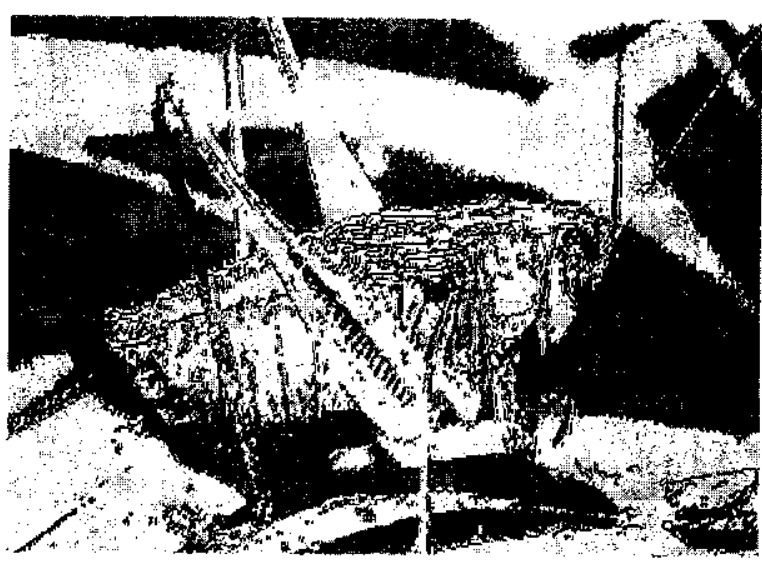

(ii)

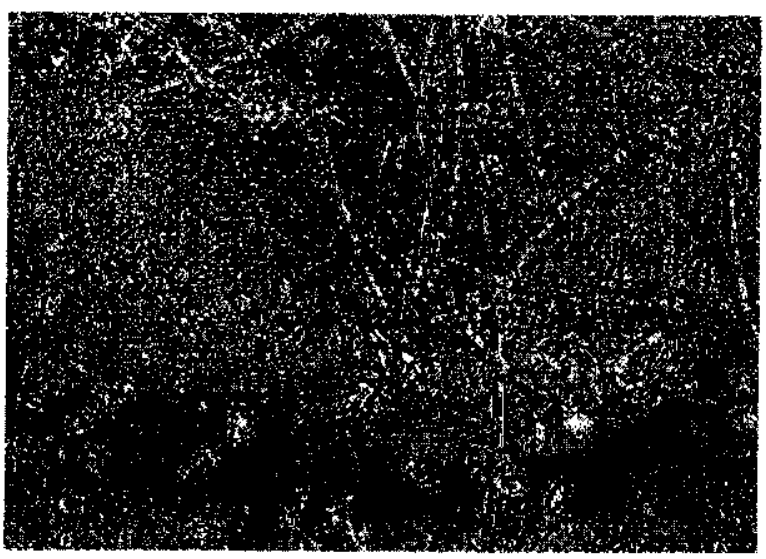

$(c)$

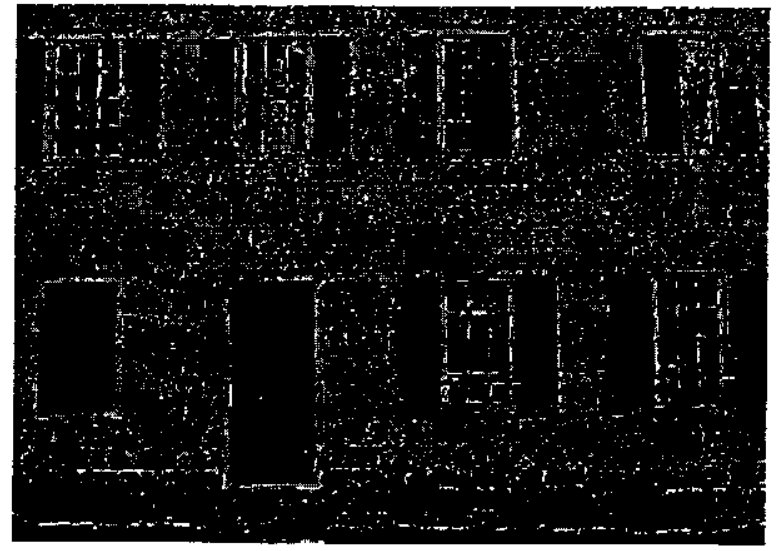

(b)

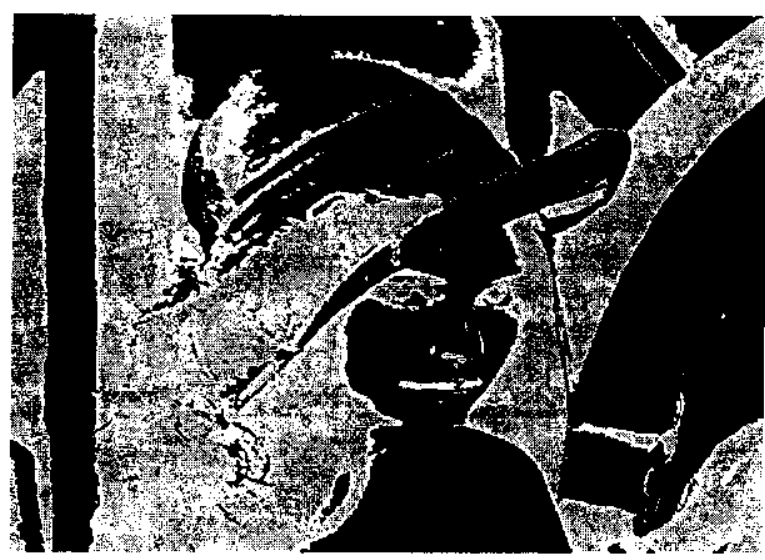

(d)

Figura 8.11: Resultades de segmentiação de inagens utilizando o modelo EICAMM aplicado às imagens pré-processadas. Todas imagens foram pré-processadas pela metodologia proposta e scgmentadias polo modulo EICAMM para $k=5$ classes. (a) resultado para imagem I1; (b) resultado para imagom 12; (c) resultado para imagem I3; (d) resultado para imagern I4.

É possivel notar, com excegão do resultado para a imagem 12, que o algoritmo k-médias năo obteve resultados satisfatórios de segmentação, detectando algumas regiöes nitidamente homogêneats conno se fosscm regiöes distintas. Essa observação leva a crer que o método $k$ médias nâo é láo adequado quanto o modelo EICAMM para classificação de dados de imagens. Isto pode ser olsservado comparando-se as imagens da Figura $8.10 \mathrm{com}$ as da Figura 8.12. Essc fato, na verdacle, pode estar associado a uma limitação do k-médias em relação a modolos baseados em ICA, que são capazes de detectar estrul uras estatísticas importantes cm dados de imagens. 


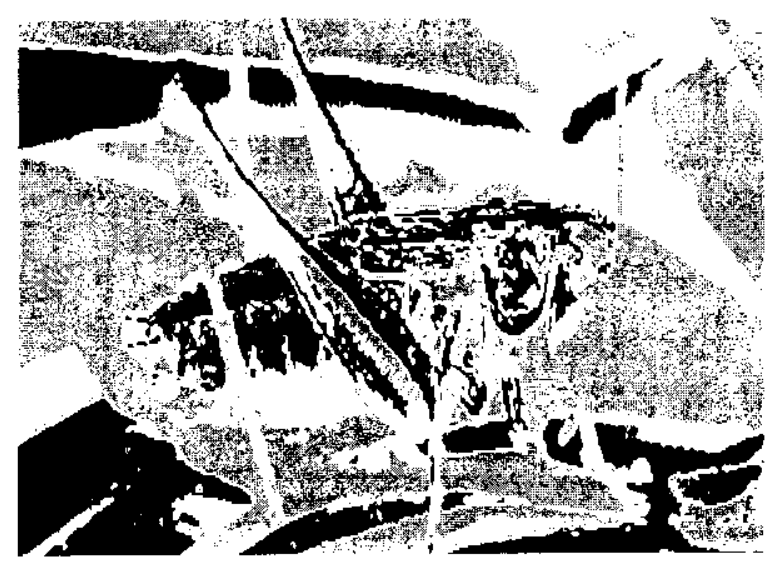

(a)

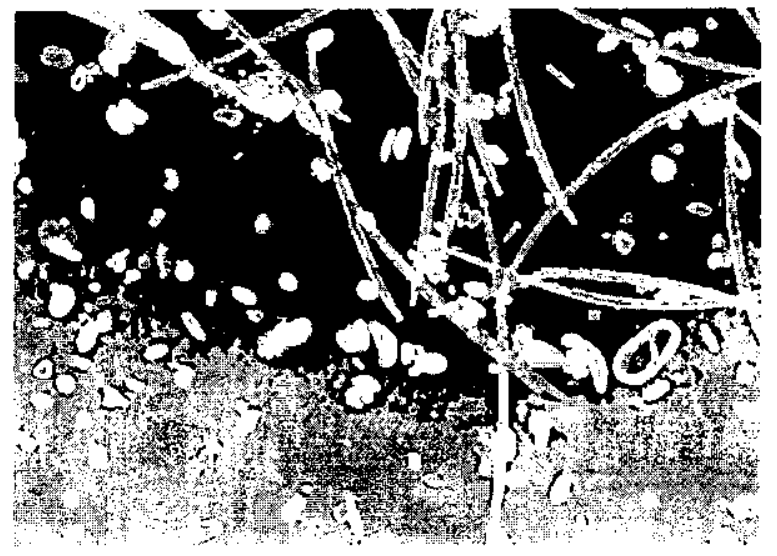

(c)

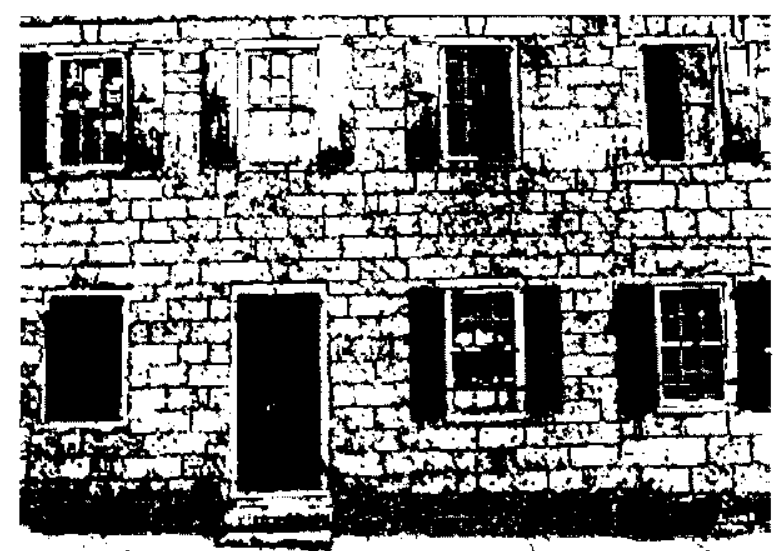

(b)

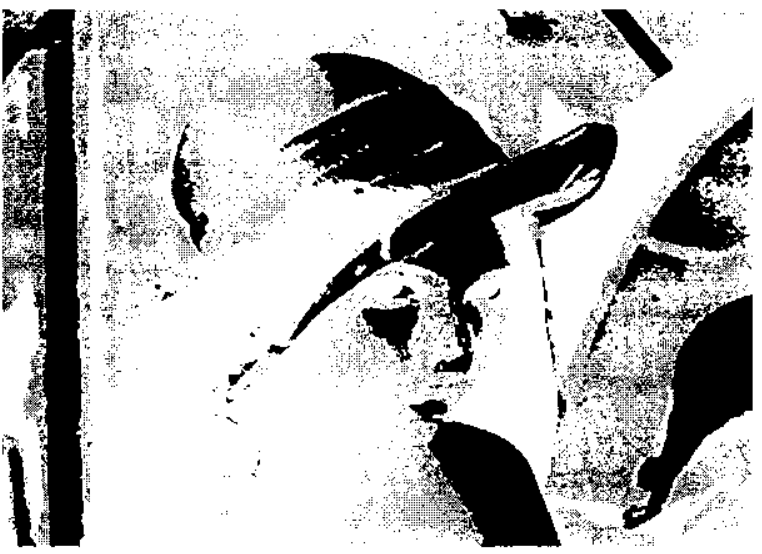

(d)

Fignura 8.12: Resultados de segmentaçăo de imagens utilizando o algoritmo k-médias aplicado às imagens pré-processadas. 'Jodas imagens foram pré-processadas pela metodologia proposta e segmentaulas pelo algoritmo $k$-médias para $k=3$ classes. (a) resultado para irnagem I1; (b) resultado para imagem I2; (c) resultado para imagem I3; (d) resultardo para imagem 14 .

\subsection{Considerações Finais}

Neste capítulo, foram apresentados e discutidos os resultados experimentais envolvendo os modelos auto-organizáveis considerados neste trabalho e a metodologia proposta para segmentação de imagens.

Primeiramente, para avaliar o desempenho do modelo EICAMM. proposto neste trabalho. foram realizadas análises comparativas con o método ICAMM o o algoritmo k-médias, para conjuntos de dados simulados e o conjunto Iris. Dessas análises, observon-se que o EICAMM 
oldeve resultades nowhores que o h-médias, para todos os experimentos com mais de duas classes o o modelo ICAMM apresenton uma ronvergencia muito lenta, com rosultados de classificação insatisfatórios.

Nos experimentos parn segmentaçăo de imagens, a eliciência da metodologia de préprecessamento proposta foi avaliada em resultados obtidos pela rede neural Fuzzy ART o o modelo EICAMM, tendo levado a resultidos melhores do que os resultados obtidos com as imagens originais, om todos os casos.

Os melhores resultades de segmentaçäo obtidos neste trabalho referem-so à ut ilizaçäo do modelo EICAMM, aplicado as imagens pró-processadas pela metodologia proposta, o que vem a clemonstrar a cficiôncia da unificação das duas principais contribuiçoes originais desta tese. 


\section{Capítulo 9}

\section{Conclusões e Trabalhos Futuros}

\subsection{Principais Considerações}

A scgmentação automática de imagens ainda é considerada, em muitas aplicaçōes, uma tarefa difícil, embora vários nétodos para esse propósito tenham sido desenvolvidos. Dentre as várias abordagens consideradas para a segmentação de imagens, aquclas que utilizam técnicas inteligentes possuem algumas vautagens em relação aos métodos clássicos, sendo capazes de tratar as incertezas increntex às informações contidas em imagens, ou de aprender a partir dos dados existentes e da combinaỹa destes com algum conhecimento a priori. Alguns métodos inteligentes apresentam. além destas características, a capacidade de auto-organização, ou seja, são modelos capazes de alurnder sem que haja um controle externo exercido pelo ambiente.

Modelos de redes nemais artificiajs possuem propriedades que os tornam adequados para a aplicaçāo em processamento de imagens. Dentre estas, destacam-se o processamento adaptativo e a caparidade de auto-organização e generalização. No Capítulo 3, foram apresentadas as técnicas de análisc multivariada PCA c técnicas de agrupamento, juntamente com modelos de redes neurais auto-organizáveis que implementam essas técnicas. A rede neural PCA Adaptativa foi utilizada em expcrimentos preliminares no presente trabalho para compressâo de imagens médicas, tendo obtido êxito em aproximar os resultados obtidos pela técnica clássica e superar aqueles obtidos pola técnica JPEG. O modelo neural Fuzzy ART implementa uma técnica de análise de agrupamento baseada na teoria de conjuntos nebulosos, tendo sido utilizado nos experimentos de segmentaşào do inagens deste trabalho.

Un dos problemas abordados nesta tese refere-se à sensibilidade que a rede Fuzzy ART apresenta no processamento do dados com ruídos. Portanto, quando este modelo é aplicado a tarefas de segmentação. é interessante considerar métodos de pré-processamento que pos- 
sam, dentre outras funcionalidades. reduzir os ruídos presentes nas imagens a screm processadas pelo modelo. Com esse oljetivo, foi proposta, no Capitulo 5, uma metodologia para pré-processamento de imagens. que combina o método Sparse Code Shrinkage para redução de ruídos c suavização da imagem c o detcctor de bordas de Sobel, que tem a funçāo de restaurar as bordas que foram borradas pclo processo de suavização. Nos experimentos apresentados na Seção 8.4.3 pode-se observar que, de fato, a metodologia de pré-processamento proposta levou a uma melhoria nos resultados de segmentaçāo obtidos pela rede Fuzzy ART.

O outro modelo auto-organizável considerado neste trabalho foi o modelo ICAMM, originalmente proposto para a classificaçāo nāo supervisionada de um conjunto de dados, nos quais a modelagem das classes baseia-se no método ICA, considerado como uma generalização da técnica PCA. Para que este modelo pudesse ser compreendido em detalhes, definiçôes e fundamentos do método ICA, juntamente com as principais abordagens ICA desenvolvidas, foram apresentados no Capitulo 4. Este cajítulo tambóm é importante para o entendimento do método Sparse Code Shrinkage, uma vez que este também relaciona-se com a técnica ICA.

Apesar de algumas caracteristicas promissoras do ICAMM terem sido reportadas no seu trabalho original. no presente trabalho, apesar das inúmeras tentativas, não foi obtido sucesso na reprodução dos resultados experimentais obtidos pelos seus autores. Como pode ser observado nos experimentos com dados simulados c com o conjunto de dados de flores Iris, apresentados nas Sç̧ōes 8.2 e 8.3. o ICAMM apresentou uma convergência muito lenta e resultados de classificação insatisfatórios.

Com o objetivo de melhorar o desempenho do ICAMM, no presente trabalho foi proposto o modelo EICAMM. derivado a partir de algumas modificaçóes realizadas no ICAMM, considerando-se aspectos de modelagem e implementação. Uma das contribuições mais importantes é a incorporaçào do método de otimização de Levenberg-Marquardt ao algoritmo de aprendizagem, para garantir e melliorar a convergência do modelo.

Para avaliar o desempenho do EICAMM, este foi aplicado a tarefas de classificação não supervisionada de dados simulados com distribuições rıão gaussianas para diferentes dimensões e número de classes, assim como na classificação do bem conhecido conjunto de dados Iris. Objetivando ainda uma discussão mais abrangente, os resultados obtidos pelo método proposto foram comparados com outros obtidos pelo método ICAMM na sua forma original e pelo método $k$-médias, para a classificação dos mesmos conjuntos de dados. Essas análises comparativas iniciais, apresentadas nas Seçoes 8.2 e 8.3, mostraram que o método EICAMM supera o $k$-médias nos experimentos com mais de duas classes para dados simulados. Assim, o método pode ser considerado um bom classificador, o que pode ser comprovado pela análise visual dos resultados, 
em que se avaliou o comportanento auto-organizável do método EICAMM, comparado ao do k-médias.

Finalmente. unificando as duas principais contribuições originais desta tese, na Seção 8.4.3, o método EICAMN é utilizado na segmentação de imagens originais c das mesmas imagens pré-processadas pela metodologia proposta neste trabalho. Um dos pontos ressaltados na análise desses experimentos é cue o modelo EICAMM de fato é adequado para classificação de dados de imagem, tendo obtidos resultados significativamente melhores do que aqueles obtidos pela rede neural Fizzy ART. Pode-se notar que, ao contrário do modelo ncural, o EICAMM conseguiu detectar regiōes lomogeneas que representam estruturas (objetos) importantes presentes nas imagens. Além disso, assim como nos experimentos com a rede Fuzzy ART, foi possível observar como a utilizaçào da metodologia de pré-processamento proposta levou a resultados de segmentação significativamente melhores do que aqueles obtidos quando a técnica é aplicada às imagens originais.

\subsection{Principais Contribuições}

Os resultados desta tesc representam contribuiçōes $\mathrm{cm}$ duas linhas de pesquisa: modelos auto-organizáveis e técnicas de segnentação de imagens. As principais contribuiçōes são:

- A demonstraçáo de que o método Sparse Code Shrinkage, proposto originalmente para reduçăo de rúdos, lambém pode ser utilizado como um filtro de suavização de imagens, fazendo con que as regiôes de interesse fiquem mais homogêneas.

- O descnvolvimento de una metodologia para pré-processamento de imagens, que combina - método Sparsé Code Shrinkage, para reduçāo de ruídos e suavização da imagem, e o detector de bordas de Sobel, que tem a função de rcstaurar as bordas que foram borradas pelo processo de suavização.

- A demonstragáo de que os resultados apresentados em (Lee, Lewicki \& Sejnowski 2000), trabalho que introduzin o ICAMM, não podem ser reproduzidos utilizando o algoritmo de aprendizado original.

- O desenvolvimento de um modelo auto-organizável para classificação não supervisionada c segmentaçào de imagens. que, por ser bascado na técnica ICA, é capaz de detectar estruturas estatísticas importantes em dados de imagens. 
- O desenvolvimento de um sistema que unifica as propostas de metodologia para préprocessamento de imagens o o modelo auto-organizável proposto para segmentacão.

Os resultados obticlos nesta tese geraram publicações em relatório técnico (Oliveira \& Romero 2004c), artigos cu congresso nacional (Olivcira, Romero, Mazucheli \& Nonato 2000), internacional (Oliveira, Vicent.in \& Romero 2002) (Oliveira \& Romero 2004a), (Oliveira \& Romero 2004b) e um artigo submetido à revista intcrnacional (Vicentiri, Oliveira \& Romero 2004).

\subsection{Propostas de Trabalho Futuros}

Uma vez que o modelo ICAMM, na sua forma original, é baseado em uma abordagem neural, uma extensão natural do modelo EICAMM é a construção de uma rede neural que incorpore esse método. Nissa proposta, a rede neural a ser construída receberia, como entrada, os dados a screm classificados, retornando, como saída, valores que indiquem a classe a que cada padrâo foi associado. de acordo com as probabilidades condicionais calculadas pelo método. As regras para atualizaça dos pesos da rede seriam portanto baseadas de alguma forma no algoritmo de aprendizado derivado para o EICAMM.

Outras funçoos de ativação näo lineares também podem ser avaliadas para serem incorporadas ao método EICAMM. no lugar da função tangente hiperbólica, considerada neste trabalho.

Na tentativa de acelerar ainda mais a convergência do modelo EICAMM, o método FastICA, que apresenta a projuriedade de rápida convergência, pode ser utilizado para a estimação das matrizes de base: do modelo, ao invés da abordagem infomax.

Para uma avaliação mais profunda do método EICAMM na classificação não supervisionada de dados simulatos e outros conjuntos numéricos, variando o número de classes e as dimensões dos dados, propõo-se a utilização de técnicas de validaçāo de agrupamentos (Jain \&: Dubes 1988). A justificativa para essa proposta é que estas técnicas devem ser mais adequadas para avaliar o desempenho de modelos auto-organizáveis do que o método de validação cruzada, que requer um monitomanento dos rótulos ao longo do processo de classificação, além do conhecimento prévio do mímero de classes e dos rótulos verdadeiros dos dados.

Outra idéia interessitnte é avaliar a utilizaçāo de outros tipos de funções objetivo a serem otimizadas na derivaçăo do método EICAMM. Algumas sugestões podem estar relacionadas a funções baseadas na teoria de conjuntos nebulosos ou na estatística bayesiana. 


\section{Apêndice A}

\section{Derivação das Regras de}

\section{Aprendizado ICA}

\section{A.1 Para uma Rede Neural com uma Entrada e uma Saída}

\section{A.1.1 Função de Transferência Logística}

A funçào de transferencia logística é dada pela seguinte equação não linear:

$$
y=\frac{1}{1+e^{-u}}, \quad u=w x+w_{0}
$$

$\Lambda$ derivada parcial de y em relação à $u$ ć calculada da seguinte forma:

$$
\begin{aligned}
y^{\prime} & =\frac{\partial y}{\partial u}=\frac{1}{\left(1+e^{-u}\right)^{2}} e^{-u}=\frac{1}{\left(1+e^{-u}\right)}\left(\frac{e^{-u}}{1+e^{-u}}\right)= \\
& =\frac{1}{\left(1+e^{-u}\right)}\left(1-\frac{1}{\left(1+e^{-u}\right)}\right)=y(1-y) .
\end{aligned}
$$

Para a derivaçấo da regra de aprendizado para $w$, a seguinte equaçāo diferencial deve ser resolvida:

$$
\Delta w \propto \frac{\partial H}{\partial u !}=\frac{\partial}{\partial w}\left(\ln \left|\frac{\partial y}{\partial x}\right|\right)=\left(\frac{\partial y}{\partial x}\right)^{-1} \frac{\partial}{\partial u}\left(\frac{\partial y}{\partial x}\right)
$$

sendo que, neste caso, y é a função logística, apresentada na Equação (A.1).

Tem-se que: 


$$
\begin{aligned}
\frac{\partial y}{\partial x} & =\frac{\partial y}{\partial u} \frac{\partial u}{\partial x}=y(1-y) w=w y(1-y), \\
\frac{\partial y}{\partial u^{\prime}} & =\frac{\partial y}{\partial u} \frac{\partial u}{\partial u}=y(1-y) x=y x(1-y), \\
\frac{\partial}{\partial x}(y(1-y)) & =\frac{\partial y}{\partial x}(1-y)+y\left(\frac{-\partial y}{\partial x}\right)= \\
& =u y(1-y)(1-y)-y w y(1-y) \quad \text { (usando }(\text { A.4)) }= \\
& =u y(1-y)(1-y-y)= \\
& =u y(1-y)(1-2 y) .
\end{aligned}
$$

Logo:

$$
\begin{aligned}
\frac{\partial}{\partial u}\left(\frac{\partial y}{\partial x}\right) & =\frac{\partial}{\partial x}\left(\frac{\partial y}{\partial u}\right)= \\
& =\frac{\partial}{\partial x}(y x(1-y)) \quad(\text { usando }(A .5))= \\
& =y(1-y)+x \frac{\partial}{\partial x}(y(1-y))= \\
& =y(1-y)+x w y(1-y)(1-2 y) \quad \text { (usando(A.6)) }= \\
& =y(1-y)(1+u x(1-2 y)) .
\end{aligned}
$$

Dividindo a Equaçắ (A.7) pela Equação (A.4), obtém-se:

$$
\Delta w=\frac{1}{w}+x(1-2 y)
$$

Para a derivação da regra de aprendizado para $w_{0}$, a seguinte equação diferencial deve ser resolvida:

$$
\Delta w_{0} \times \frac{\partial H}{\partial w_{0}}=\frac{\partial}{\partial w_{0}}\left(\ln \left|\frac{\partial y}{\partial x}\right|\right)=\left(\frac{\partial y}{\partial x}\right)^{-1} \frac{\partial}{\partial w_{0}}\left(\frac{\partial y}{\partial x}\right)
$$

sendo que, neste caso. y ć a função logística, apresentada na Equação (A.1).

Tem-se que:

$$
\frac{\partial y}{\partial u_{0}}=\frac{\partial y}{\partial u} \frac{\partial u}{\partial w_{0}}=y(1-y)
$$


Dessa forma.

$$
\begin{aligned}
\frac{\partial}{\partial w_{0}}\left(\frac{\partial y}{\partial x^{\prime}}\right) & =\frac{\partial}{\partial x}\left(\frac{\partial y}{\partial w_{0}}\right)= \\
& =\frac{\partial}{\partial x}(y(1-y)) \quad(\text { usando }(A .10))= \\
& =\frac{\partial y}{\partial x}(1-y)+y\left(\frac{-\partial y}{\partial x}\right)= \\
& =w y(1-y)(1-2 y) .
\end{aligned}
$$

Dividindo a Equaçào (A.11) pcla Equação (A.4), obtém-se:

$$
\Delta w_{0}=1-2 y
$$

\section{A.2 Função de Transferência Tangente Hiperbólica}

A funçăo de transforencina tangente hiperbólica é dada pela seguinte equação não linear:

$$
y=\tanh (u), \quad u=w x+w_{0}
$$

A derivada parcial de $y$ em relação à $u$ é calculada da seguinte forma:

$$
y^{\prime}=\frac{\partial y}{\partial u}=\operatorname{sech}^{2}(u)=1-\tanh ^{2}(u),=1-y^{2}
$$

Para a derivaçăo da regra de aprendizado para $w$, a seguinte equação diferencial deve ser resolvida:

$$
\Delta w \times \frac{\partial H}{\partial u}=\frac{\partial}{\partial w}\left(\ln \left|\frac{\partial y}{\partial x}\right|\right)=\left(\frac{\partial y}{\partial x}\right)^{-1} \frac{\partial}{\partial w}\left(\frac{\partial y}{\partial x}\right)
$$

sendo que, neste caso, y ć a função tangente hiperbólica, apresentada na Equação (A.13).

Tern-se que:

$$
\begin{aligned}
& \frac{\partial y}{\partial x}=\frac{\partial y}{\partial u} \frac{\partial u}{\partial x}=\left(1-y^{2}\right) w \quad(\text { usando }(A .14)) \\
& \frac{\partial y}{\partial u}=\frac{\partial y}{\partial u} \frac{\partial u}{\partial w}=\left(1-y^{2}\right) x
\end{aligned}
$$




$$
\begin{aligned}
\frac{\partial}{\partial w}\left(\frac{\partial y}{\partial x}\right) & =\frac{\partial}{\partial x}\left(\frac{\partial y}{\partial w}\right)= \\
& =\frac{\partial}{\partial x}\left(\left(1-y^{2}\right) x\right) \quad(\text { usando }(A .17))= \\
& =\left(1-y^{2}\right)+x \frac{\partial}{\partial x}\left(\left(1-y^{2}\right)\right) \quad \text { (usarudo a regra do produto para derivada) }= \\
& =\left(1-y^{2}\right)+x(-2 y) \frac{\partial y}{\partial x}= \\
& =\left(1-y^{2}\right)-2 x y\left(1-y^{2}\right) w= \\
& =\left(1-y^{2}\right)(1-2 x y w) .
\end{aligned}
$$

Dividindo a Equaşăo (A.18) pela Equaçāo (A.16), obtém-se:

$$
\Delta w=\frac{1}{w}-2 x y
$$

Para a derivaçào da regra de aprendizado para $u_{0}$, a seguinte equação diferencial deve ser resolvida:

$$
\Delta u_{0} \times \frac{\partial H}{\partial u_{0}}=\frac{\partial}{\partial u_{0}}\left(\ln \left|\frac{\partial y}{\partial x}\right|\right)=\left(\frac{\partial y}{\partial x}\right)^{-1} \frac{\partial}{\partial u_{0}}\left(\frac{\partial y}{\partial x}\right)
$$

sendo que, neste caso, y ć a função tangente hiperbólica, apresentada na Equação (A.13).

Tem-se que:

$$
\frac{\partial y}{\partial u_{0}}=\frac{\partial y}{\partial u} \frac{\partial u}{\partial w_{0}}=\left(1-y^{2}\right)
$$

Dessa format:

$$
\begin{aligned}
\frac{\partial}{\partial u_{0}}\left(\frac{\partial y}{\partial r}\right) & =\frac{\partial}{\partial x}\left(\frac{\partial y}{\partial w_{0}}\right)= \\
& =\frac{\partial}{\partial x}\left(1-y^{2}\right) \quad(\text { usando }(A .21))= \\
& =-2 y \frac{\partial y}{\partial x}= \\
& =-2 y\left(1-y^{2}\right) w .
\end{aligned}
$$

Dividindo a Equação (A.22) pela Equação (A.16), obtém-se: 


$$
\Delta w_{0}=-2 y
$$

\section{A.3 Para uma Rede Neural com $N$ Entradas e $N$ Saídas}

Como W é uma matriz quadrada e $g$ é uma funçāo inversível, a fdp multivariada de y pode ser escrita cono (Papoulis 1991):

$$
p_{\mathbf{y}}(\mathbf{y})=\frac{p_{\mathbf{x}}(\mathbf{x})}{|\mathbf{J}|}
$$

onde $|\mathbf{J}|$ é o valor absoluto da matriz Jacobiana de derivadas parciais (ver Equação (2.7)). Esse valor pode ser simplificado plo produto entre o determinante da matriz de pesos e as derivadas; $y_{i}^{\prime}$. das saidas. $y_{i}$ em relaçào às entradas da rede:

$$
\mathbf{J}=(\operatorname{det} \mathbf{W}) \prod_{i=1}^{N} y_{i}^{\prime} .
$$

Por exemplo, no caso da funçào sigmóide logística:

$$
\begin{aligned}
& y_{i}=\frac{1}{1+e^{-u_{i}}}, \\
& y_{i}^{\prime}=y_{i}\left(1-y_{i}\right) .
\end{aligned}
$$

A funçäo a ser maximizada, no caso da abordagem infomax, refere-se à entropia conjunta da saída, dada por:

$$
\begin{aligned}
H(\mathbf{y}) & =-E\left[\ln p_{\mathbf{y}}(\mathbf{y})\right]= \\
& =E[\ln \mid \mathbf{J} !]-E\left[\ln p_{\mathbf{x}}(\mathbf{x})\right] \quad \text { (usando a relação em (A.24)) }
\end{aligned}
$$

Aqui, os pesos devem ser ajustados para maximizar $H(\mathbf{y})$. Nesse caso, as mudanças aplicadas aos pesos en W só irão afetar o termo $E[\ln |\mathbf{J}|]$ na Equação (A.28). Portanto, substituindo a Equação (A.25) na Equaçiò (A.28), resulta em:

$$
\Delta \mathbf{W} \times \frac{\partial H}{\partial \mathbf{W}}=\frac{\partial}{\partial \mathbf{W}} \ln |\mathbf{J}|=\frac{\partial}{\partial \mathbf{W}} \ln |\operatorname{det} \mathbf{W}|+\frac{\partial}{\partial \mathbf{W}} \ln \prod_{i}\left|y_{i}^{\prime}\right| .
$$


Uma vez que det $\mathbf{W}=\sum w_{i j}$ cof $u_{i j}$, para qualquer linha $i$, tem-se, para um único peso $w_{i j}$ :

$$
\frac{\partial}{\partial u_{i j}} \ln |\operatorname{det} \mathbf{W}|=\frac{\operatorname{cof} w_{i j}}{\operatorname{det} \mathbf{W}}
$$

Para a derivada em relaçào à matriz completa $\mathbf{W}$, utiliza-se a definiçào de uma matriz inversa e o fato que a matriz adjunta, adj $\mathbf{W}$, é a transposta da matriz de cofatores. Isso resulta em:

$$
\frac{\partial}{\partial \mathbf{W}} \ln |\operatorname{det} \mathbf{W}|=\frac{(\operatorname{adj} \mathbf{W})^{T}}{\operatorname{det} \mathbf{W}}=\left[\mathbf{W}^{T}\right]^{-1} .
$$

Para o segundo termo na Equação (A.29), nota-se que o produto, $\ln \prod_{i}\left|y_{i}^{\prime}\right|$, divide-se em uma somatória de termos logarítmicos, sendo que somente um desses termos depende de um particular $w_{i j}$. O cálculo da derivada correspondente a esse termo depende da função não linear utilizada para a transferência de informaŗão e procede de maneira análoga ao caso de uma rede neural com una midade. 


\section{Referências}

Amari, S. \& Cardoso, J.-F. (1997). Blind source separation semiparametric statistical approach. IEEE Transactions on Signal Processing 45(11), 2692-2700.

Amari, S., Cichocki, A. \& Yang. H. (1996). A new learning algorithm for blind signal separation. Advances in Ncural Information Processing Systerns 8, 757-763.

Amari, S. (1998). Natural gradient works efficiently in learning. Neural Computation 10(2), $251-276$.

Bachelder. 1. Waxmm. A. \& Seibert, M. (1993). A neural system for mobile robat visual place learning and recognition. In In Proceedings of the World Congress on Neural Netuorks, Volume I. Hilisdale. pp. 512 517. NJ: Erlbaum Associates.

Back, A. D. \& Weigend, A. S. (1997). A first application of independent component analysis to extracting structure from stock returns. International Journal of Neural Systems 8, 473484.

Baraldi. A. \& Alpayclin. E. (1998). Simplified ART: A new class of ART algorithms. Technical Report TR-98-004. Intemational Computer Science Institute, Berkeley, CA.

Baraldi, A. \& Alpaydin. E. (20)2a). Constructive fecdforward ART clustering networks - part I. IEEE Transaction on Neural Networks 13(3), 645-661.

Baraldi. A. \& Alpaydin, E. (2002b). Constructive feedforward art clustering networks - part II. IEEE Transaction on Neural Networks 13(3), 662-677.

Baraldi, A. \& Blondia. P. (1999). A survey of fuzzy clustering algorithms for pattern recognition. II. IEEE Transactions on Systems, Man and Cybernetics, Part B (Cybernetics) 29, $786-801$.

Barlow, H. B. (1961). Possible principles underlying the transformation of sensory messages. In W. A. Rosenblith (Ed.), Sensory Communication. MIT Press. 
Barlow. H. B. (1994). What is the computation goal of the neocortex? In C. Koch \& J. I. Davis (Eds.), Large-sicale neuronal theories of the brain. Cambridge, MA: MIT Press.

Bartfai, G. (1994). Hierarchical clustcring with ART neural networks. In IEEE International Conference on Neural Networks, Volume 2, pp. 940-944. IEEE Press.

Bartfai, G. (1995). An ART-based modular architecture for learning hicrarchical clusterings. TR CS-TR-95/3.

Bazaraa. M. S. (1979). Nonlineenr Programming. John Wiley \& Sons.

Bell, A. J. \&. Sejnouski. T. J. (1995). An information-maximization approach to blind separation and blind deconvolution. Neural Computation 7(6), 1129-1159.

Bell, A. J. \& Sejnowski, T. J. (1997). The 'independent components' of natural scenes are edge filters. Vision Research 37, 33273338.

Bell, A. \& Scjnowski, T. (1996). Learning higher-order structure of a natural sound. Network: Computation in Neural Systems 7, 261-266.

Bernardon. A. M. \& Carrick. J. E. (1995). A neural system for automatic target learning and recognition applied to hare and camoulflaged sar target. Neural Networks 8, 1103-1108.

Bhandarkar, S., Kol, J. \& Suk. M. (1997). Multi-scale image segmentation using a hierarchical self-organizing feature map. Neurocomputing 14(3), 241-272.

Bishop, C. M. (1994). Mixture density networks. Neural Computing Research Group Report NCRG/94/004. Aston Universily.

Blake, C. L. \& Merı, C. J. (1998). UCI repository of machine learning databases. University of California. Irvine, Dept. of Information and Computer Sciences. http://www.ics.uci.chu/ mlearn/MLRepository.html.

Cardoso, J.-F. (1997). Infomax and maximum likelihood for blind source separation. IEEE Signal Processing Letters 4(4), 112 114.

Cardoso, J.-F. (1998). Blind signal separation: Statistical principles. Proceedings of IEEE $86(10), 20092025$.

Carpenter, A. \& Grossberg, S. (1990). Art 3 hierarchical search: Chemical transmitter in selforganizing pattern recognition architectures. Neural Networks 3, 129-152.

Carpenter, G. A., Grossberg: S., Markuzon, N., Reynolds, J. H. \& Rosen, D. B. (1991). Fuzzy ARTMAP: A neural network architecture for incremental supervised learning of analog multidimensional maps. Technical report, Boston University. 
Carpenter, G. A., Grossberg. S. \& Rosen, D. B. (1991a). ART2-A: An adaptive resonance algorithm for rapid category learning and recognition. Neural Networks 4. 493-504.

Carpenter, G. A., Grossherg. S. \&: Rosen, D. B. (1991b). Fuzzy ART: fast stable learning and categorization of analog patterns by an adaptive ressonance system. Neural Networks 4 , 759771.

Carpenter, G. A., Grossberg, S. \& Rosen, D. (1991c). Fuzzy ART: Fast stable learning and ${ }^{\text {? }}$ categorization of analog patterns by an adaptive resonance system. Neural Networks 4 . $759-771$.

Carpenter, G. A. \& Grossberg. S. (1987a). Art2: self-organization of stable category recognition codes for analog inpul patterns. Applied Optics 26(23), 4919-4930.

Carpenter, G. A. \& Grossberg. S. (1987b). A massively paralle] architecture for a selforganizing neural pattern recognition machine. Computer Vision, Graphics and Image Processing 37, 54 1.15.

Carpenter, G., Grossbesg. S.. Neurophysiol, C. \& Reynolds, S. (1991). Artmap: Supervised real-time lcarning and classification of nonstationary data by a sclf-organizing neural network. Neural Netuorks 4. 565-588.

Caudell. T. P. \& Healy. M. J. (1994). Adaptive resonance theory networks in the encephalon autonomous vision systcm. In Proceedings of the IEEE International Conference on Neural Networks. Orlando. Florida, pp. 1235-1240. IEEE.

Changeux, J. P. \& Danchin. A. (1976). Selective stabilization of developing sinapses as a mechanism for the specification of neural networks. Nature 264, 705-712.

Cheng. H. \& Bomman. (. A. (2001). Multiscale Bayesian segmentation using a trainable context modicl. IEEE Transaction on Image Processing 10(4), 511-525.

Cichocki, A.. Unbchaum. R. \& Rummert, E. (1994). Robust learning algorithm for blind separation of signals. Eletronics Letters $30(17), 1386-1387$.

Comon, P. (1994). Independent component analysis-a new concept? Signal Process 36, $287-314$

Cormack, R. M. (1971). A review of classification. Journal of the Royal Statistical Society $134(3), 321 \cdots 367$.

Cover, T. M. \& Thomis, J. A. (1991). Elements of information theory. New York: Wiley.

DeGroot, M. H. (1987). Probability and statistics, 2nd. Edition. Oxford: Addison-Wesley, Reading. 
Derrode, S., Mercier, G. \& Pieczynski. W. (2003). Unsupervised multicomponent image segmentation combining a vectorial HMC model and ICA. In Proceedings of 2003 International Conference on Image Processing (ICIP 2003), Barcelona, pp. 14-17.

Diamantaras, K. I. \& Kung, S. (1996). Principal Component Neural Networks: Theory and Applications. New York: Wiley.

Diday: E. \& Simon, J. (1980). Clustering analysis. In Digital Pattern Recognition, K.S. Fu (Editor), 47-94.

Donoho, D. L., Jonhstome. I. M., Kerkyacharian, G. \& Picard, D. (1995). Wavelet shrinkage: asymptopia? Journal of the Royal Statistical Society 57, 301-337.

Duda, R. \& Hart, P. (1973). Paftern classification and scene analysis. New York: Wilcy.

Fausset, L. (1994). Fundamental of Neural Networks: Architectures, Algorithms, and Applications. Prentice Hall.

Field, D. J. (1994). What is the goal of sensory coding? Neural Computation 6, 559-601.

Fletcher, R. (1980). Iractical Methods of Optimization. John Wiley \& Sons.

Frank. T., Kraiss, K. \& Kullen. T. (1999). Comparative analysis of fuzzy ART and ART-2A network clustering performance. IEEE Transactions on Neural Networks 9(3), 544-559.

Freman, J. A. \& Skapura, D. M. (1991). Neural Networks - Algorithms, Applications, and Programing Techniques. New York: Addison-Wesley.

Fu, K. \& Mui, J. (1981). A survey on image segmentation. Pattern Recognition 13, 3*16.

Gaeta, M. \& Lacoune, J. (1990). Sources separation without a priori knowledge: The maximum likelihood solution. Proceedings of Eusipco 90,621-624.

Gan, K. \& Lua. K. (1992). Chinese character classification using an adaptive resonance network. Pattern Recognition. 25, 877888 .

Girolami, M. \& Fyfe, C. (1997). Generalized independent component analysis through unsupervised learning with emergent Bussgang properties. Proceedings of International Conference on Neural Networks 3: 1788-1791.

Girolami, M. (1998). An alternative perspective on adaptive independent component analysis algorithms. Neural Computation 10(8), 2103-2114.

Gonzalez, R. C. \& Woods, R. E. (1992). Digital image processing. Reading, MA, USA: Addison-Wesley.

Graybill, F. A. (1983). Matrices with applications in statistics, 2nd edition. Belmont. 
Grossman, S. I. (1986). Multivariate Calculus, Linear Algebra and Differential Equations. Academic: Press.

Guo, G. \& Ma, S. (200(0). Bayesian learning, global competition and unsupervised image segnentation. Pattem Recognition Letters 21, 107-116.

Ham, F. \& Han, S. (1996). Classification of cardiac arrhythmias using fuzzy artmap. IEEE Transactions on Bionedical Engineering 43(4), 425-430.

Haykin. S. (1994). Noural netuorks - A comprehensive foundation. New Jersey: Macmillan College Publishing Company.

Hebb, D. O. (1949). The organization of behavior: A neuropsychological theory. New York: Wiley.

Heranlt, J. \& Jutten. ( . (1986). Space or time adaptive signal processing by neural network models. Neural Networks for Computing: AIP Conference Proceedings, 206-211.

Hertz, J., Krogh. A. \& Pahner, R. (1991). Introduction to the theory of neural computation. Redwood City. CA: Addison-Wesley Publishing Compary, Inc.

He, J., Tan, A.-H. \&: Tan. C.-L. (2002). Art-c : A neural architecture for self-organization under constraints. In Proceedings of International Joint Conference on Neural Networks (IJCNN). IIawaii. USA. pp. $2550-2555$.

Hopfield. J. J. (1982). Neural networks and systems with emergent selective computational abilities. In National Academy of Sciences of the USA, Volume 79, pp. 3088-3082.

Hotelling, H. (1933). Auctysis of a complex of statistical variables into principal components. Journal of Educational P'sychology 24, 498-520.

Hussain, T. S. (1993). ARTSTAR: A supervised modular adaptive resonance network classifier. Master's thesis, Quenn's University, Kingston, Ontario - Canada.

Hyvärincn, A. Hoyer. P. \& Oja, E. (2001). Image denoising by sparse code shrinkage. In S. Haykin \& B. Kosko (Eds.), Intelligent Signal Processing. IEEE Press.

Hyvärinen, A., Karhunen, J. \& Oja, E. (2001). Independent component analysis. John Wiley \& Sons

Hyvärinen, A., Oja, E., Hoyer, P. \& Hurri, J. (1998). Image feature extraction by sparse coding and independent component analysis. Proceedings of International Conference on Pattern Recognition (ICPR'98), 1268-1273. 
Hyvärinen, A., Oja. E. \& Hover. P. (1998). Sparse code shrinkage for image denoising. In Proc. IEEE Int. Joint Conf. on Neural Networks, pp. 859-864.

Hyvärincn, A. \& Oja. E. (1997). A fast fixed-point algorithms for independent component analysis. Ncural Computation 9(7), 14831492.

Hyvärinen, A . (1998). New approximations of differential entropy for independent component analysis and projection pursuit. In Advances in Neural information processing systems, Volume 10. Cambridge, MA: MIT Press.

Hyvärinen. A. (1999). Fast and robust fixed-point algorithms for independent component analysis. IEEE Transactions on Neural Networks 10(3), 626-634.

Jain, A. K., Duin. R. P. W. \& Nao. J. (2000). Statistical pattern recognition: A review. IEEE Transactions on Pattern Analysis and Machine Intelligence 22(1), 4-37.

Jain, A. \& Dubes. R. (1988). Algorithms for Clustering Data. Prentice Hall.

Jain, A. (1989). Fundamentals of Digital Image Processing. Prentice Hall.

Johnson, R. A. \& Wichern. D. W. (1998). Applied multivariate statistical analysis. Englewood Cliffs, NJ: Prentice-Hall. Inc:

Jung, T.-P., Makeig. S., Mckeown, M. J., Bell, A., Lee, T.-W. \& Sejnowski, T. J. (2001). Imaging brain dymamics using independent component analysis. Proceedings of the IEEE $89(7) .1107 \cdot 1122$.

Jutten, C. \& Herault, J. (1991). Blind separation of sources, part I: An adaptive algorithm based on neuronimetic architecture. Signal Processing 24, 1-10.

Karayianmis, N. B. \& Pai. P. (1999). Segmentation of magnetic resonance images using fuzzy algorithms for learning vector quantization. IEEE Transactions on Medical Imaging 18(2). $172-180$.

Karhunen, J. \& Joutsensalo, J. (1994). Representation and separation of signals using nonlinear PCA type learning. Neural Networks 7, 113-127.

Kendall, M. \& Stuart. A. (1958). The advanced theory of statistics. Charles Griffin \& Company.

Kendall, M. (1980). Multivariate analysis, 2nd edition. London: Griffin.

Kohonen, T. (1988). Self-organization and associative memory, 3rd edition. New York: Springer-Verlag.

Kohonen, T. (1989). Self-organization and associative memory. New York: SpringerVerlag.

Kohonen, T. (2001). Self-Organizing Maps, 3rd edition. Springer. 
Kosko, B. (1991). Neural netuorks and fuzzy systems: A dynamical approach to machine intelligence. Englewood Cliffs, New Jersey: Prentice Hall.

Lee, T.-W., Girolani, M., Bell, A. J. \& Sejnowski, T. J. (1998). A unifying informationtheoretic framowork for independent component analysis. International Journal of Mathematical and Computer Modoling 39, 1-21.

Lee, T., Girolami, N. \& Sejnowski. T. J. (1999). Independent component analysis using an extended infomax algorithm for mixed sub-gaussian and super-gaussian sources. Neural Computation 11(2), 409433

Lee, T., Lewicki. M. S. \& Sejnow'ski, T. J. (2000). ICA mixture models for unsupervised classification of non-gatuswian classes and automatic context switching in blind signal separation. IEEE Transactions on Pattern Analysis and Machine Intelligence 22(10), 10781089.

Lewicki, M. \& Scjnowski. T. J. (2000). Learning overcomplete representations. Neural Computation $12.337-365$.

Linsker, R. (1992). Local syaptic learning rules suffice to maximize mutual information in a linear network. Neural Computation 4, 691-702.

Lo. Y. S. \& Pei. S. C. (1999). Color image segmentation using local histogram and selforganization Kohoucn map. Proceedings of IEEE International Conference on Image Processing. Kobe. Japan. 232-235.

Lu, C. \& Liao. H. (2000). Oblivious cocktail watermarking by sparse code shrinkage: A regional- and global-based scheme. In IEEE Intcrnational Conference on Image Processing: Volume 3. Vancouver, Caniada. pp. $13 \cdots 16$.

MacQucen. J. (1967). Some methods for classification and analysis of multivariate observations. Proccedings of the Fifth Berkeley Symposium on Mathematical statistics and probability $1,281 \cdot 297$.

Makeig, S., Jung, T.-P'. Bell, A. J.. Ghahramani, D. \& Sejnowski, T. (1997). Blind separation of auditory event-related brain responses into independent components. Proceedings of National Academy of Sciences (USA) 94, 10979-10984.

Manly, B. (1986). Multivariate statistical methods: A primer. London: Chapman and Hall.

Mao, J. \& Jain, A. (1995). Articial neural networks for feature extraction and multivariate data projection. IEEE Transactions on Neural Networks 6(2), 296-317.

Martínez, A. M. (2000). Recognition of partially occluded and/or imprecisely localized faces using a probabilistic approach. Computer Vision and Pattern Recognition 1, 712-717. 
Masters, T. (1995). Adnonced algorithms for neural networks. New York: Wiley.

Mehta, B., Vij, L. \& Rabelo, L. (1993). P'rediction of secondary structures of proteins using fuzzy artmap. In World Congress on Neural Networks - WCNN'93, Portland, OR, pp. $228-232$.

Mendel, J. M. \& Nclaren. R. W. (1970). Reinforcement-learning control and pattern recognition systcms. Adaptive, Learning, and Pattern Recognition Systems: Theory and Application.s. Acadenic Press, New York, 287-318.

Minsky, M. L. (1961). Steps towards artificial intelligence. Proceedings of the Institute of Radio Engineers 49. 8-30.

Moghaddam, B. \& Pent land, A. (1996). Probabilistic visual learning for object representation. Early Visual Leorning. S. Nayar and T. Poggio (eds.), Oxford University Press, 99-130.

Moya. M., Koch. M. \& Hostctler. L. (1993). One-class classifier networks for target recognition applications. In World Congress on neural networks - WCNN'93, Portland, OR, pp. 797801.

Murase, H. \& Nayar. S. (1995). Visual learning and recognition of 3d objects from appearance. International Journal of Computer Vision 14, 5-24.

Nadal. J. P. \& Parga. N. (1994). Non-linear neurons in the low-noise limit: A factorial code maximizes infonnation transfer. Network 4, 2953312.

Nakamura, T. \& Ogasawara, T. (1999). On-line visual learning method for color image segmentation and object tracking. In International Conference on Intelligent Robots and Systems (IROS' 99). Pp. 222 228. IEEE.

Nathan, R. (1970). Spatial freçuency filtering. In B. S. Lipkin \& A. Rosenfeld (Eds.), Picture Processing and Psychopictorics, pp. 151-164. New York: Academic Press.

Nayar, S., Nene. S. \& Murase, H. (1995). Subspace methods for robot vision. IEEE Transactions on Robotics and Automation 12(5), 750-758.

Nelson, M. (1992). The dnta compression book. New York: M \& T Books.

Nodes, T. A \& Gallagher, N. C. (1982). Median filters: some manipulations and their properties. IEEE Transactions on on Acoustics, Speech and Signal Processing 30(5), 739-746.

Oja, E., Kiviluoto. K. \& Măläroiu, S. (2000). Independent component analysis for financial time series. Proceedings of IEEE Symposium on Adaptive Systems for Signal Processing, Communications, and Control (AS-SPCC'OO), 111-116. 
Oja. E. (1997). The nonlincar PCA learning rule in independent component analysis. Neurocomputing $17: 25 \cdot 45$.

Oliveira, P. R. \& Romcro, R. A. F. (2004b). Enhanced ICA mixture model for image segmentation. International Confcrence on Machine Learning and Applications (ICMLA'2004), Lonisville. USA.

Oliveira, P. R. \& Romero, R. A. F. (2004a). Enhanced ICA mixture model for unsupervised classification. In IX Ibero-American Conference on Artificial Intelligence (IBERAMIA'2004). Mexico. pp. 205-214.

Oliveira, P. R. \& Romero. R. A. F. (2004c). Modelo de Misturas ICA Aperfeiçoado para Classificação Nâo Supervisionada. NOTAS ICMC-USP- 82, Sāo Carlos, SP.

Oliveira, P. R.: Romero. R. F.. Mazucheli, J. \& Nonato, L. G. (2000). Techniques for image compression: A comparative analysis. Proceedings of the 6th Brazilian Symposium on Neural Netnortis. Rio de Janeiro, Brazil, 249-254.

Oliveira, P. R., Vicentin. J. F. \& Romero, R. A. F. (2002). Combining fuzzy art network clustering and sparse code shrinkage for image segmentation. Proceedings of 9th International Conference on Neural Information Processing (ICONIP'2002) 5, 2435-2439. Singapore.

Oliveira. P. R. (1997). Redes neurais artficiais para extração de características. ICMCUniversidade de São Paulo (USP), São Carlos, Brasil: Dissertação de Mestrado.

Olshausen, B. A. \& Ficld. D. J. (1996). Emergence of simple-cell receptive field properties by learning a sparse code for natural images. Nature 381, $607-609$.

Olshausen, B. A. \& Ficld. D. I. (1997). Sparse coding with an overcomplete basis set: A estrategy employed by V1? Vision Research 37, 3311-3325.

Pao, Y. H. (1989). Adaptive pattern recognition and neural networks. New York: Addison Wesley.

Papoulis, A. (1991). Probability, random variables and stochastic processes. New York: McGraw-Hill.

Pearlmutter, B. \& Parra. L. (1996). A context-sensitive generalization of ICA. Proceedings of ICONIP:96, 151-157.

Pearson, K. (1894). Contributions to the mathematical theory of evolution. Philosophical Transactions of the Royal Society A 185(71), 71-110.

Pearson, K. (1901). On lines and planes of closest fit to systems of points in space. Philosophical Magazine 2, 559 572 . 
Peper, F., Zhang. B. \& Noda, H. (1993). A comparative study of ART2-A and the selfOrganizing feature map. In Proceedings of 199.3 International Joint Conference on Neural networks (IICNN'93), Volume 2, Nagoya, pp. 1425-1429.

Pham, D. L. \& Prince, J. L. (1999). Adaptive fuzzy segmentation of magnetic resonance images. IEEE Transactions on Medical Imaging 18(9), 737-752.

Pham, D. T. \& Garrat, P. (1997). Blind separation of mixture of independent sources through a quasi-maximm likelihood approach. IEEE Transactions on Signal Processing 45(7), $1712-1725$.

Pham, T. D. \& Yan. H. (1999). Color image segmentation using fuzzy integral and mountain clustering. Fuzzy Sets and Systems 107(2), 121-130.

Pratt, W. K. (2001). Digital image processing, 3rd edition. New York: John Wiley and Sons.

Racz. J. \& Dubrawshi. A. (1995). Artificial neural network for mobile robot topological localization. Robotics and Autonomous Systcms 16, 73-80.

Ridder, D. D., Kittler, J. \& Duin, R. (2000). Probabilistic PCA and ICA subspace mixture models for image segmentation. In M. Mirmehdi \& B. Thomas (Eds.), Proceedings of British Machine Vision Conference 2000 (BMVC 2000), Bristol, UK, pp. 112-121.

Roth, Z. \& Baram. Y. (1996). Multidimensional density shaping by sigmoids. IEEE Transactions on Neural Networks $7(5), 1291 \cdot 1298$.

Rubner, J. \& Tavan. P. (1989). A self-organizing network for principal-component analysis. Europhysics Letters 10, 693-698.

Rumelhart, D.. Hilton. G. E. \&. Williams, R. J. (1980). Learning internal representations by error propagation. In Parallel Distributed Processing, Volume 1: Foundations, Cambridge. MIT Press.

Russ, J. C. (1992). The image processing handbook. Boca Raton, FL: CRC Press.

Sahoo, P.. Soltani, S.. Wong, A. \& Chen, Y. (1988). A survey of thresholding techniques. Vision Graphics Image I'rocessing 41, 233-200.

Sanger, T. (1989). Optimal unsupervised learning in a single layer feedforward network. Neural Networks 12, 459-473.

Seibert, M. \& Waxulan, A. M. (1992). Adaptive 3-d object recognition from multiple views. IEEE Transactions on Pattern Analysis and Machine Intelligence 14(2), 107-124. 
Seibert, M. \& Waxmaı. A. (1993). An approach to facc recognition using saliency maps and caricatures. In Procectings of the World Congress on Neural Networks (WCNN-93), Volume III. Hillsdale pp. 661-664. NJ:Earlbaum.

Shah. C. A., Arora. M. K., Rolbila. S. A. \& Varshney, P. K. (2002). ICA mixture nodel based unsupervisesl classification of hyperspectral imagery. Proceedings of 31st Applied Imagery Pattern Recognition Workshop. Washington, ELA, 112-121.

Simoncelli, E. P. \& Adelson. E. H. (1996). Noise removal via bayesian wavelet coring. In Third IEEE International Conference on Inage Processing, Lausanne, Switzerland.

Soliz, P. \& Donohoe, G. W. (1996). Adaptive resonance theory neural network for fundus image segmentation. In N.J.Earbbaum (Ed.). In Proceedings of the World Congress on Neural Neturorkis HCNN96. Hillsdale, pp. 11801183.

Suzuki, Y. (1995). Solf-organizing QRS-wave recognition in ECG using neural networks. IEEE Transactions on Netural Netuorks 6(6), 14691477.

Szatmáry, B.. Szirtes. G. \& Lörincz, A. (2003). Robust hierarchical image representation using non-negative matrix factorisation with sparse code shrinkage preprocessing. Pattern Analysis and Applications 6(3), 194-200.

Tasciyan, T. A.. Beckniamn. C. F.. Morris, E. D. \& Smith, S. M. (2001). ICA-based segmentation of the brain on perfusion data. In In Proceedings of $23 r d$ Annual International Conference of the IFEE Engineering in Medicine and Biology Society, pp. 126-130.

Traina Jr., C.. J. M. Trainit, A., Seeger, B. \& Faloutsos, C. (2000). Slim-trees: High performance metric trees minimizing overlap between nodes. In Proceedings of 7th Intemational Conference on Extending Database Technology - EDBT, Konstanz, Germany, pp. 51-65.

Vicentini, J. F., Oliveira, P. R. \& Romero, R. A. F. (2004). Investigating the potential of ART neural network models for indexing and information retrieval (submetido). International Journal of Intelligent Systems, 19 páginas.

Vicentini, J. F. (2002). Indexação e Recuparaçào de Informaçōes Utilizando Redes Neurais da Familia ART. ICUC: Lniversidade de São Paulo (USP), Sāo Carlos. Brasil: Dissertaçāo de Mestrado.

Vigário, R., Särelä, J., Jousmäki, V., Hämäläinen, M. \& Oja, E. (2000). Independent component approach to the analysis of EEG and $M E G$ recordings. IEEE transactions on biomedical engineering $47(5), 589-593$. 
Wactlar, H., Kanade. T.. Smith, M. \& Stevens, S. (1996). Intelligent access to digital video: Infomedia project. IEEF, Computer 29(3), 46--52.

Walker, J. S. (1999). A primer on wavelets and their scientific applications. Chapman and Hall.

Watanabe, S. (1985). Pattern recognition: human and mechanical. New York: Wiley.

Waxman, A. M.. Scibert, M. C., Gove, A., Fay, D. A., Bernardon, A. M., Lazott, C., Stcele, W. R. \& Cumingham, R. K. (1995). Neural processing of targets in visiblc, multispectral IR and SAR imagery. Neural Networks 8(7-8), 1029-1051.

Weiss, S. \& Kulikowski. C. A. (1991). Computer Systems That Learn. Morgan Kaufmann.

Weng. J. J. (1996). Cresceptron and shoslif: Toward comprchensive visual learning. Early Visual Lraming. S. K. Nayar and T. Poggio (eds.), Oxford University Press, 183 214.

Williamson. J. R. (1996). Ganssian artmap: A neural network for fast incremental learning of moisy multidinemisional maps. Neural Networks 9(5), 881-897.

Xu, L. (1993). Lcast MSE reconstruction: A principle for self-organizing nets,. Neural Netuorks 6. 627-648.

Yao, K. C., Mignotte. M.. Collet. C., Galerne, P. \& Burel, G. (2000). Unsupervised segmentation using a solf-organizing map and a noise estimation in sonar imagery. Pattern Recognition 33. 1575158.

Zadeh. L. A. (1994). Fuxyy logic. neural networks, and soft computing. Communications of the ACM 37(3), 7784 .

Zhang, M., Hall. L. O. \& Goldgof. D. B. (2002). A generic knowledge-guided image segmentation and labeling system using fuzzy clustering algorithms. IEEE Transactions on Systems, Man. and Cybonetics, Part B 2(5), 571-582. 